\title{
Inaugural-Dissertation
}

zur Erlangung der Doktorwürde der Naturwissenschaftlich-Mathematischen Gesamtfakultät der Ruprecht-Karls-Universität Heidelberg

vorgelegt von

Diplom-Mathematiker Volker Reichenberger

aus Bruchsal

Tag der mündlichen Prüfung: 20. Januar 2004 



\section{NUMERICAL SIMULATION OF MULTIPHASE FLOW IN \\ FRACTURED POROUS MEDIA}

Gutachter: Prof. Dr. Peter Bastian

Prof. Dr. Gabriel Wittum 



\section{Preface}

This work is an interdisciplinary effort and would not have been possible without many contributions from different sources. My gratitude goes to:

$\triangleright$ Peter Bastian who laid the basis to this work in many different ways and always supported me. I couldn't think of a better teacher of the art of scientific computing.

$\triangleright$ Gabriel Wittum for recognizing the importance of scientific software development, his support and for sharing my Macintosh enthusiasm.

$\triangleright$ Rainer Helmig generously supported my work. Working with him and his group was always a pleasure.

$\triangleright$ The other members of the UG development team for their work: Peter Bastian, Klaus Birken, Klaus Johannsen, Stefan Lang, Nicolas Neuß, Henrik Rentz-Reichert, Christian Wieners.

$\triangleright$ Hartmut Jakobs, my colleague in Braunschweig and Stuttgart. Howdy, and I'm looking forward to the next time we get pulled over by the Police on a lonely Texas highway.

$\triangleright$ Lina Neunhäuserer found and fixed errors in the fracture discretization module. The meshes for the single-phase flow fractured domain were created by her.

$\triangleright$ Annette Hemminger created the fractured-domain generator and generously gave it to me to produce the domains for the three-dimensional simulations.

$\triangleright$ Special thanks to the other members of the Heise-Reload-Club. Olaf Ippisch is the best proof reader I know. 
Preface 


\section{CONTENTS}

Preface 5

I. INTRODUCTION II

2. The Model of Fluid Flow in Fractured Porous Media

2.I. Material properties ..................... I3

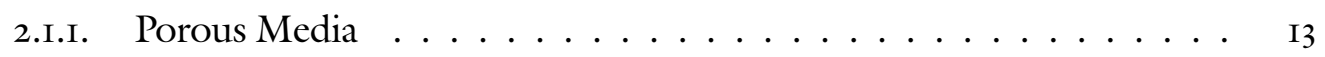

2.I.2. Length scales in the subsurface $\ldots \ldots \ldots \ldots \ldots$ I4 . . . . . . . . . . . .

2.I.3. Types of Discontinuities in Porous Media . . . . . . . . . . I5

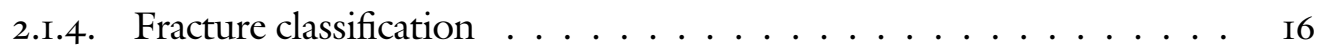

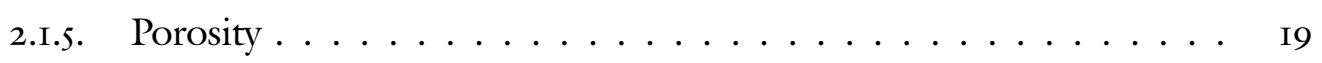

2.I.6. Representative Elementary Volume. . . . . . . . . . . . . . 20

2.I.7. Heterogeneity and Anisotropy . . . . . . . . . . . . . 22

2.2. Single phase fluid flow in fractured porous media . . . . . . . . . . 23

2.2.I. Darcy's law for flow of a single fluid phase in a porous medium . . . 23

2.2.2. Single phase fluid flow in a fracture . . . . . . . . . . . 25

2.3. Multiphase flow at the microscopic scale . . . . . . . . . . . . . . 26

2.3.I. Capillary forces and the shape of the capillary surface . . . . . . . . 26

$2.3 .2 . \quad$ Capillary pressure $\ldots \ldots \ldots \ldots \ldots \ldots 28 \ldots \ldots \ldots$

2.3.3. Capillary behavior in the pore space and in fractures . . . . . . 28

2.3.4. Dynamic processes at the microscale . . . . . . . . . . . . . . . . . . . . . . . . .

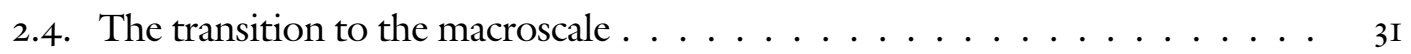

2.4.I. Saturation ........................ 3 I

2.4.2. Multiphase flow equations . . . . . . . . . . . . . . . . . . 32

2.4.3. Capillary pressure functions . . . . . . . . . . . . . . . . . . . . 32

2.4.4. Relative permeability functions . . . . . . . . . . . . . . . . . . 34

2.4.5. The model for two-phase flow in porous media . . . . . . . . . . 35

2.4.6. Interface conditions at Media Discontinuities . . . . . . . . . 36

2.5. Models for Fractured Porous Media . . . . . . . . . . . . . . . . . 37

2.5.I. Equivalence Models . . . . . . . . . . . . . . . . . . 38

2.5.2. Discrete Modeling and Numerical Simulation . . . . . . . . . . . . 40

2.6. Approach taken in this work . . . . . . . . . . . . 4I 


\section{Contents}

3. Finite Volume Discretization ANd Multigrid Solution 43

3.I. Numerical solution schemes for the two-phase equations . . . . . . . . . . . 43

3.2. Character of the equations . . . . . . . . . . . . . 46

3.3. Numerical methods for hyperbolic and degenerate parabolic equations . . . 47

3.3.I. Nonlinear hyperbolic equations . . . . . . . . . . . . . 47

3.3.2. Degenerate parabolic equations . . . . . . . . . . . . . . . . . 49

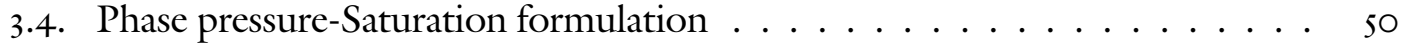

3.5. The Finite Volume Method $\ldots \ldots \ldots \ldots$ 5I

3.5.I. Geometry of the problem and the Primary and Secondary Mesh. . . 5I

3.5.2. The Approximation Spaces . . . . . . . . . . . . . . . . 54

3.5.3. Weak formulation . . . . . . . . . . . . . . . 56

3.5.4. Implementation . . . . . . . . . . . . . . . 58

3.6. Time discretization $\ldots \ldots \ldots \ldots \ldots \ldots \ldots \ldots \ldots$

3.7. Nonlinear System Solution . . . . . . . . . . . . . . . . . . 6I

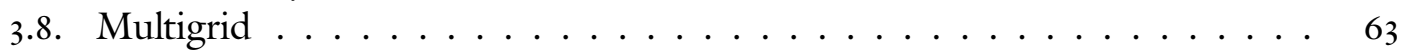

3.8.I. The Multigrid Algorithm . . . . . . . . . . . . . . . . . 66

3.8.2. Robust Multigrid Algorithms . . . . . . . . . . . . . . . . . . . . . 67

3.8.3. Parallel and Adaptive Multigrid . . . . . . . . . . . . . . . 68

3.8.4. Multigrid for Systems $\ldots \ldots \ldots \ldots$. . . . . . . . . . . . . . . . . . . .

3.8.5. Truncated restriction for discontinuous problems . . . . . . . . 7I

3.9. Computer Program Implementation . . . . . . . . . . . . . 7I

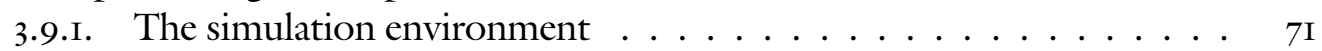

3.9.2. The numerical framework $\mathrm{UG}$. . . . . . . . . . . . . . . . 72

3.9.3. Pre- and post-processing . . . . . . . . . . . . . 74

4. The DisCONTINUOUS GALERKIN METHOD FOR ELLIPTIC PROBLEMS 79

4.I. $\quad$ Development of the Discontinuous Galerkin Method . . . . . . . . . . . . 79

4.2. Formulation of the bilinear form $\ldots \ldots \ldots \ldots \ldots$

4.3. Multigrid Solution . . . . . . . . . . . . . . . . . . 86

4.4. Numerical Results for the DG Method . . . . . . . . . . . . . . . . . 88

4.4.I. Single Phase Flow in Homogeneous Media . . . . . . . . . . . . 88

4.4.2. Single Phase Flow in Heterogeneous Media . . . . . . . . . . . . . . . . . 89

4.5. Single Phase Flow in Fractured Media . . . . . . . . . . . . . . . . 92

\begin{tabular}{lll}
\hline 5. & NUMERICAL EXPERIMENTS & 97
\end{tabular}

5.I. $\quad$ Vertical water-gas flow . . . . . . . . . . . . . . . . . . . . . . . . . . 97

5.2. Well simulation . . . . . . . . . . . . . . . . . . . I06

5.3. Three-dimensional Oil-Water simulation . . . . . . . . . . . . I08

5.4. Three-dimensional Gas-Water simulation . . . . . . . . . . . . . . III

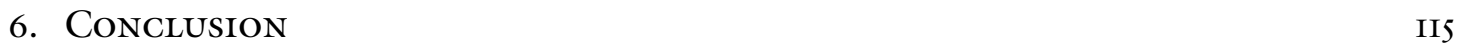

\begin{tabular}{|l}
\hline A. The Hele-Shaw Analog for Viscous Flow in Parallel Plates \\
in
\end{tabular} 


\section{CONTENTs}

\begin{tabular}{ll}
\hline B. INTEGRAL TRANSFORMATIONS I23 & I23
\end{tabular}

B.I. Two-dimensional elements in two space dimensions . . . . . . . . . . . . . I23

B.2. One-dimensional elements in two space dimensions . . . . . . . . . . . . . I24

B.3. Lower-dimensional elements in three space dimensions . . . . . . . . . I25 
Contents 


\section{INTRODUCTION}

One major difficulty in the numerical simulation of multiphase flow in the subsurface arises from the strongly heterogeneous and anisotropic nature of soils. Thin layers of different material traverse the ground, from impermeable granite to loose sandstone. Layers stretch over kilometers in horizontal direction, while being only meters or less thick.

If anisotropic layers represent a difficulty for the numerical simulation, fractures aggravate the problem. Only millimetres or centimeters thick, the can stretch over kilometers. Their high conductivity for fluids makes it impossible to ignore them in the simulation process, but the numerical simulation of fluid flow and transport processes in these structures is a challenge that has became accessible only in the recent years in the field of scientific computing. The task is demanding from different viewpoints, since models for fluid flow in fractures and fractured porous media are still being developed and their implementation in computer programs requires the combination of sophisticated efficient techniques. Bringing together these fields in an interdisciplinary work is a challenging, but instructive experience.

The structure of this work reflects the structure of the modeling process for simulation in porous media, as outlined in Ewing (I983) for reservoir simulation. The first step is the development of a physical model. The model should include as much physical detail as necessary to describe the essential phenomena. This requires to recognize what the essential phenomena of a physical process are. The physical model should then be able to model these phenomena appropriately. Based on the physical model, the next step in the modeling process is the derivation of a mathematical formulation. In the case of multi-phase flow, the mathematical formulation is a system of nonlinear partial differential equations. The character of the mathematical model is important for the appropriate selection or development of a numerical model, because properties of the mathematical model need to be understood. Under ideal circumstances, existence, uniqueness and regularity of the solution can be verified for the mathematical model, although this is a tough requirement for systems of nonlinear partial differential equations. The devised numerical scheme should be accurate and stable, and it should be able to reproduce the distinguishing physical features of the physical model. Obviously, the numerical scheme should not introduce artificial effects into the solution. Finally, the numerical model should be implemented in a computer program, which should be correct and efficient.

If possible, the results of the computer program should be compared to experimental results. If the results do not compare well, one should iterate through all four stages of the modeling process and make changes in all the stages of the intermediate models. We would like to stress that the last point, the development of the computer program, is equally important as the other intermediate stages, and does not consist of a simple formula translation or transformation of the equations into an algorithmic language. The development of software for the numerical simulation of multi-phase flow in complex domains requires its own 


\section{INTRODUCTION}

engineering methods.

Chapter 2 will clarify what we mean when we speak of a porous medium, what fractures are - their characteristic properties - and what the equations that govern fluid flow of one or more phases in fractured porous media are. In chapter 3, we present a fully coupled, fully implicit vertex-centered finite volume scheme for the numerical solution of two-phase flow in fractured porous media which is capable of handling compressible gas-water flow on unstructured meshes with mixed-dimensional element types. In chapter 4 we explore a recent development, the discontinuous Galerkin method. There we retreat to the more simple model of single phase flow in fractures which are resolved by volumetric elements, but present multigrid results and a comparison to the finite volume method. Chapter 5 presents results obtained with the finite volume method for two-phase flow and is intended to prove the scheme handles the physical complexity in an adequate and efficient way.

Readers acquainted with numerical simulation of multiphase flow will find the key components in section 2.6, where we lay out the assumptions we impose on the fluid flow model, section 3.5 where a finite volume scheme for two-phase equations is presented. Chapter 4 about the discontinuous Galerkin method, reports on a relatively new development in subsurface modeling. 


\section{The Model of Fluid Flow in Fractured Porous MEdia}

This chapter describes how a mathematical model is derived from the physical principles that govern multiphase flow in porous media. We begin our investigation with a close look at porous media and its constituents, starting with individual grains and pores up to large scale structures like sedimentary layers and faults. The classification of fractures and a summary of their common properties lay the ground for the model assumptions that we employ for our model of two-phase flow in fractured porous media.

The transition between different scales introduces the principle of the representative elementary volume which allows for the description of fluid flow equations on an averaged scale which doesn't consider individual pores but rather statistically averaged quantities. Based on this tool, we present the model for single phase flow in porous media and consider flow in fractures as a special case. Then we extend the model to two-phase flow by first considering the additional forces at the small scale where flow phases are clearly distinguishable and then extending the averaging principle to two-phase flow. We present the concepts that are acquainted by this transition and discuss their consequences. We conclude the chapter with the presentation of the system of partial differential equations which build the basis for the model and discuss some consequences for fractures and general discontinuities in fractured media.

Further discussion of the material related to porous media in this chapter can be found in Bear (1972), Helmig (I997), and Bastian (I999); fractured media are the subject of Singhal and Gupta (I999), Bear et al. (I993), and Sahimi (I995).

\section{I. MATERIAL PROPERTIES}

Porous media have a complex structure and a clear distinction of the different scales and of the features at these scales are essential for an understanding of flow processes therein. Without considering fluid flow yet, we can identify properties of porous media which will turn out to be essential for the derivation of the fluid flow model.

\section{I.I. POROUS MEdiA}

While an intuitive understanding of the meaning of the term "porous medium" is simple, a rigorous definition is more difficult. A definition of a porous medium that exceeds the notion of a "solid with holes" should cover some characteristic features that are important for the derivation of the model and on which we base our further investigations. 

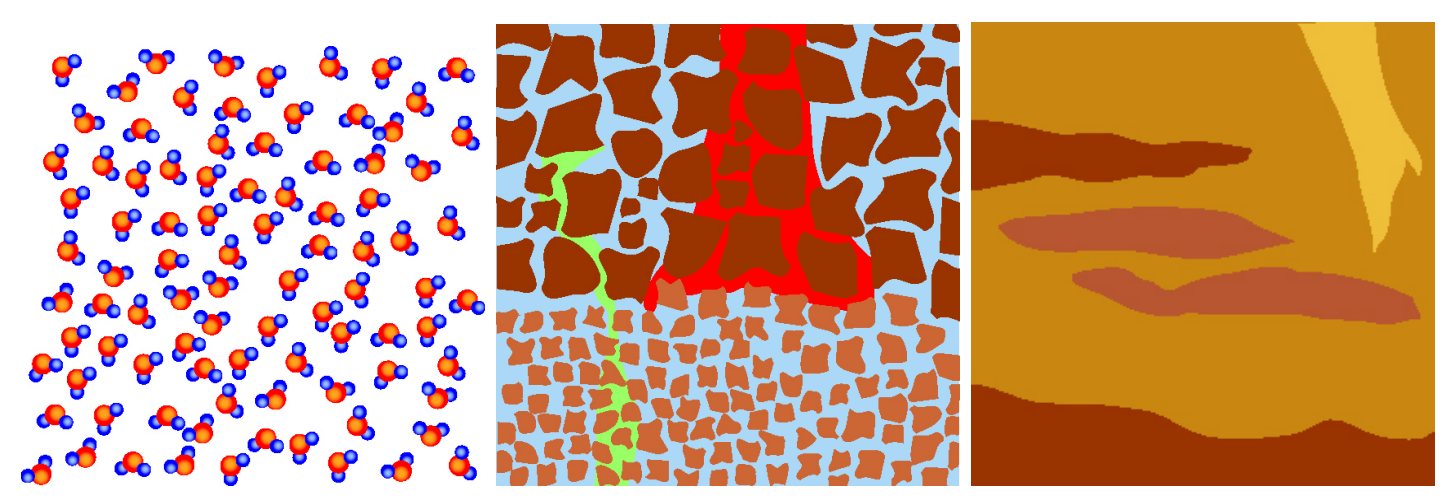

Figure 2.I.: The molecular scale (left), the microscopic scale (middle) and the macroscopic scale (right).

$\triangleright$ A porous medium is a body which consists of several phases. One of the phases is solid and is called the solid matrix. The solid phase is distributed throughout the porous medium. The other phases, which may be liquid or gaseous, occupy the complementary space. This part of the porous medium is called the pore space or void space.

$\triangleright \quad$ Large portions of the void space should be interconnected. Only the interconnected pore space is accessible to flow (it is also termed effective pore space).

Certain parts of the porous medium, though connected, may be ineffective for flow due to narrow connections or dead ends. Pores that are altogether isolated (unconnected pores) may be considered part of the solid matrix.

$\triangleright \quad$ The average pore size must be larger than the mean free path length of the fluid molecules (which is $6 \cdot 10^{-6} \mathrm{~m}$ for air at standard temperature). This assumption allows for the transition from the molecular behavior to a continuum approach on the microscale.

$\triangleright$ The average pore size must be small enough that the fluid flow is controlled by adhesive forces at the fluid-solid interfaces and by cohesive forces at the fluid-fluid interfaces in the case of multiphase flow. By this

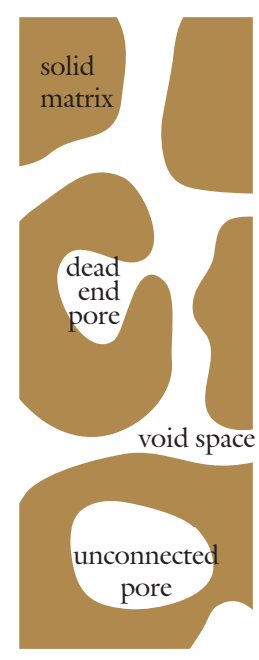
requirement networks of pipes are excluded from the model.

\section{I.2. LENGTH SCALES IN THE SUBSURFACE}

The typical scale for a porous medium of interest for waste disposal or groundwater remediation is on the scale of meters or kilometers. This is orders of magnitude above the scale at which molecular forces determine the flow. To derive a model of fluid flow at the desired scale we must distinguish the scales which constitute our medium.

On the smallest scale, at about $10^{-9} \mathrm{~m}$, the domain is resolved to individual molecules. This scale is called the molecular scale. This scale is by far too detailed for our purposes and so we employ the standard transition of continuum mechanics, where the behavior of molecules is replaced by the behavior of a hypothetical continuum on the next larger scale, 
the microscopic scale. At this scale of approximately $10^{-3} \mathrm{~m}$ individual sand grains and pore channels can be identified, and the flow behavior is determined by the Navier-Stokes equations. They are the accepted continuum model for fluid flow and have been studied extensively (Temam, I979, Doering and Gibbon, I995). These equations assume that quantities like mass density and viscosity are piecewise continuous in space and time. The NavierStokes equations together with knowledge of the pore space geometry determine the fluid flow. However, since the pore space geometry is usually not known, and because any attempt to simulate fluid flow on this scale for a region that includes more than just a few sand grains would require prohibitive amounts of computing resources, a direct numerical simulation at this scale is not feasibleף. This restriction requires the introduction of another hypothetical continuum, in which each point in the continuum is assigned to an averaged value. The averaging process (described in section 2.I.6), which employs the concept of elementary volumes, introduces new quantities (e. g. porosity and permeability) along with the macroscopic equations on the macroscopic scale. These quantities are measurable statistical properties.

It is on the macroscopic scale that media discontinuities like fractures become apparent. Layers of different materials constitute most natural porous media, and even in regions of a single material the material properties may vary significantly. Fractures are present in many rock types and can change these properties significantly.

The treatment of fractures has our special attention, so we ask what fractures are and what their distinguishing properties are when compared to other types of media discontinuities.

\section{I.3. Types of Discontinuities in Porous Media}

Fractures are not the only types of discontinuities in porous media. After Singhal and Gupta (1999) we can distinguish

$\triangleright$ Bedding planes.

$\triangleright$ Foliation (including cleavage).

$\triangleright$ Fractures (joints).

$\triangleright$ Faults and shear zones.

$\triangleright$ Other geological discontinuities.

The distinction between these classes is as follows.

BEDDING PLANES are formed by primary bedding and compositional layers. In sedimentary rocks (sandstones, siltstones,... ) they represent the most significant discontinuity. The bedding plane has a profound influence on the groundwater flow and imports anisotropy.

\footnotetext{
${ }^{\mathrm{I}}$ While the problem of determining the exact geometry of the pore space is still current, todays supercomputers have the potential to allow direct simulation of fluid flow in a region of the pore space that is of a size that is often representative for the porous medium.
} 


\section{The Model of Fluid Flow in Fractured Porous Media}

Foliation develops when platy mineral grains are aligned parallel-planar at right angles to the direction of the stress. The influence of foliation on groundwater movement is almost as big as the influence of bedding in sedimentary rocks. Bedding and foliation are the most significant discontinuities in their respective rock categories.

FrACTURES AND JOINTS The terms fracture and joint are used synonymously. Fractures are caused by a partial loss of cohesion of the rock due to stress. Fractures are of essentially planar geometry. A fracture is defined as a plane where there is hardly any visible movement parallel to the surface of the fracture. Otherwise it is defined as a fault. Generally, the term fracture is used for planar cracks or breaks in the rock without displacement. If displacement parallel to the plane can be found, the term fault (or shear) is used. Slight movement at right angles to the fracture will produce open fractures. Over time, they may get filled by secondary minerals or rock fragments. Filling materials like clay can reduce the hydraulic conductivity of fractures.

The mechanical stresses which are necessary for fracturing can not propagate across open fractures, which gives a good indication about the development history of fracture sets - if crossing fractures are encountered, it is probable that the fractures are of different age, and that one fracture developed after the other was either cemented or closed by compression.

Individual fractures have limited spatial extent and may be discontinuous in their own plane. If the lengths of the joints are greater than the spacings between them, the fractures form an interconnected continuous network. This connectivity leads to greater hydraulic conductivity of the fractured material.

See the next section for a more detailed discussion of fractures.

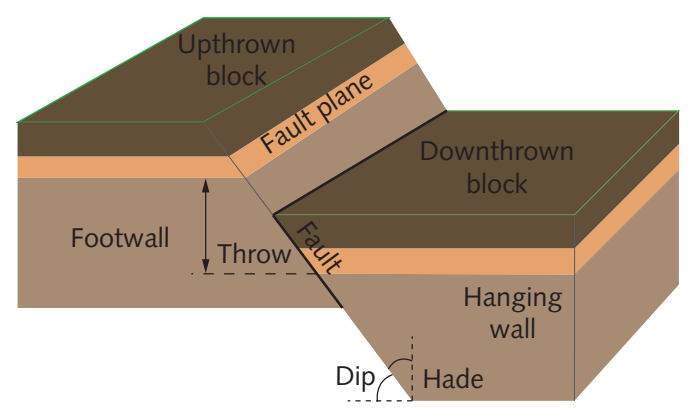

commonly used in fault description.

Shear zones are generally filled with broken and crushed rocks, possibly embedded in clay material. The classification of faults is based on slip direction. The influence of faults on groundwater flow depends on its filling: Silicified faults may act as barriers for groundwater flow, while faults filled with crushed rock may represent preferred flow paths.

\section{I.4. FRACTURE CLASSIFICATION}

The essentially planar geometry of fractures was already mentioned above. This section presents more properties that are common for fractures and will lead us to a clear definition of what properties we associate with fractures. 


\section{I. MATERIAl PROperties}

CAUSES OF Fracturing. The process by which fractures are produced are believed to be complex and are not sufficiently understood (Price and Cosgrave, I990). Some of the origins of the stresses which cause fracturing are tectonic stresses, residual stresses, construction due to shrinkage (i. e. because of cooling of magma), surficial movement (landslides, movement of glaciers), erosional unloading of deep seated rocks and weathering.

TyPes of FRACTURES. There are two broad types of fractures,

$\triangleright$ systematic fractures - which are planar and regularly distributed - and

$\triangleright$ non-systematic fractures - which are curved and irregular.

In the picture on the side systematic fractures are denoted by a and non-systematic fractures by $b$. Non-systematic fractures meet, but they do not cross other fractures. They end at the bedding surface. Their characteristic is their curvilinear pattern. A geometric classification is also possible, based on the strike of the fracture compared to the strike of the bedding or foliation or the dip of the rock. Non-systematic fractures are developed by dilation in the weathering zone.

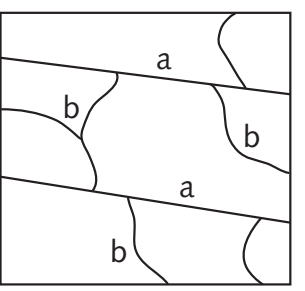

A classification based on the extent of the fractures is the distinction between first-order fractures and second-order fractures. First-order fractures cut through several layers of rocks, second-order fractures are limited to a single rock layer.

Finally, the genetic classification distinguishes between shear fractures, dilational fractures and hybrid fractures.

Fracture CHARACTERIZATION. The components of a fractured rock are

$\triangleright$ the fracture network,

$\triangleright$ the matrix, and

$\triangleright$ the fracture fillings (if present).

The characterization embodies features of the single discontinuities (like orientation, nature, persistence, aperture,...) and features of the combination of these discontinuities (e. g. a certain frequency). The characterization according to Singhal and Gupta (I999) is as follows.

Number of sets There may be several sets of fractures in the network (three or four are the most common). Relevant data has to be collected for each set.

Orientation Defines a single fracture plane in space.

Spacing The distances between joints can be very evenly distributed so that an average value can be identified.

Persistence Fracture length. The connectivity of the fracture network increases with greater average fracture length. 


\section{The Model of Fluid Flow in Fractured Porous Media}

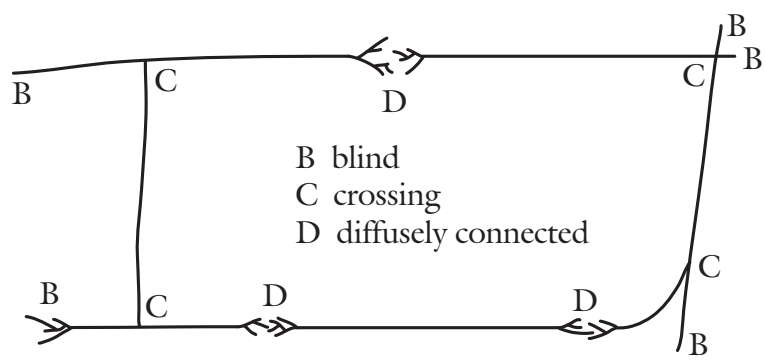

Figure 2.2.: Fracture connectivity, after Singhal and Gupta (1999).

Fracture density The density of fractures can be described with respect to length (one-dimensional measure), area or volume.

Fracture area and shape Common shapes are circular, elliptical, square, rectangular, and polygonal.

Volumetric fracture count The number of fractures per cubic meter of rock volume.

Matrix block count A matrix block unit (mbu) is the rock block bounded by a fracture network. Each matrix block unit can be considered to be independent from adjacent matrix block units.

Fracture connectivity This is an important property for flow processes. It depends on how fractures terminate: abutting, crossing or blind. Fractures that do not cross other fractures are blind. Other authors suggests a distinction into blind diffusive and connected (which includes abutting, see figure 2.2), arguing that many fractures classified as abutting are really of interfingering type (i. e. diffusive). (The first classification is from Barton et al. (I987), the second from Laubach (1992), both cited after Singhal and Gupta (1999).)

Rock quality designation The ratio of recovered core more than 4 in $(\approx 10 \mathrm{~cm})$ long and of good quality to the total drilled length. Stems from core recovery data.

Aperture Perpendicular distance separating the adjacent walls. Aperture may increase by dissolution and erosion (especially in the weathered zone). Aperture may decrease with depth due to pressure. Apertures are classified according to their size by the terms displayed in figure 2.3. Figure 2.3 shows on the left the aperture distribution of a natural fracture from the Aespö site (from Jarsjö and Geller (I996)).

Asperity Fracture walls are not smooth surfaces. The irregularities are called asperities. They reduce fluid flow and lead to channeling (preferential flow).

Wall coatings and infillings Between the walls there may be clay, fault gouge, breccia, chat, and calcite.

In section 2.5 we present a summarization of the assumptions we base on fractures for our model. 


\section{I. MAterial properties}

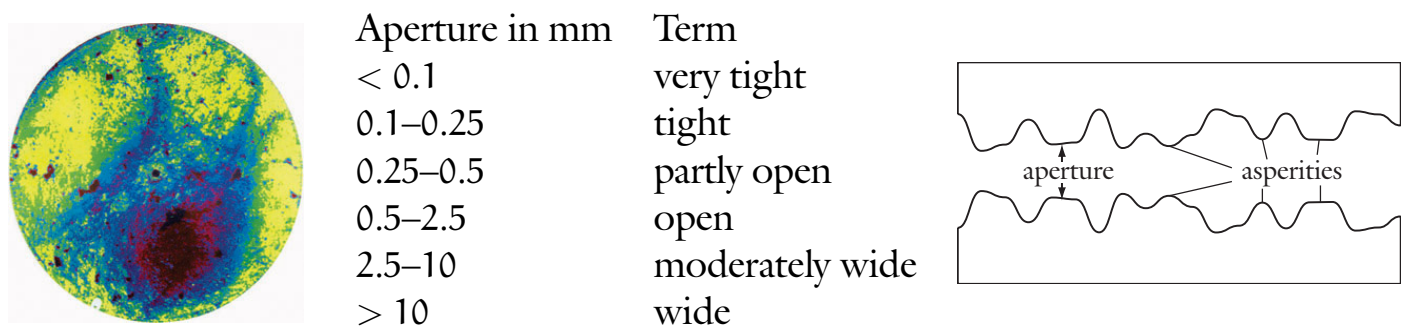

Figure 2.3.: Aperture distribution for a fracture from the Äespö site, aperture classification, and definition of asperity and aperture.

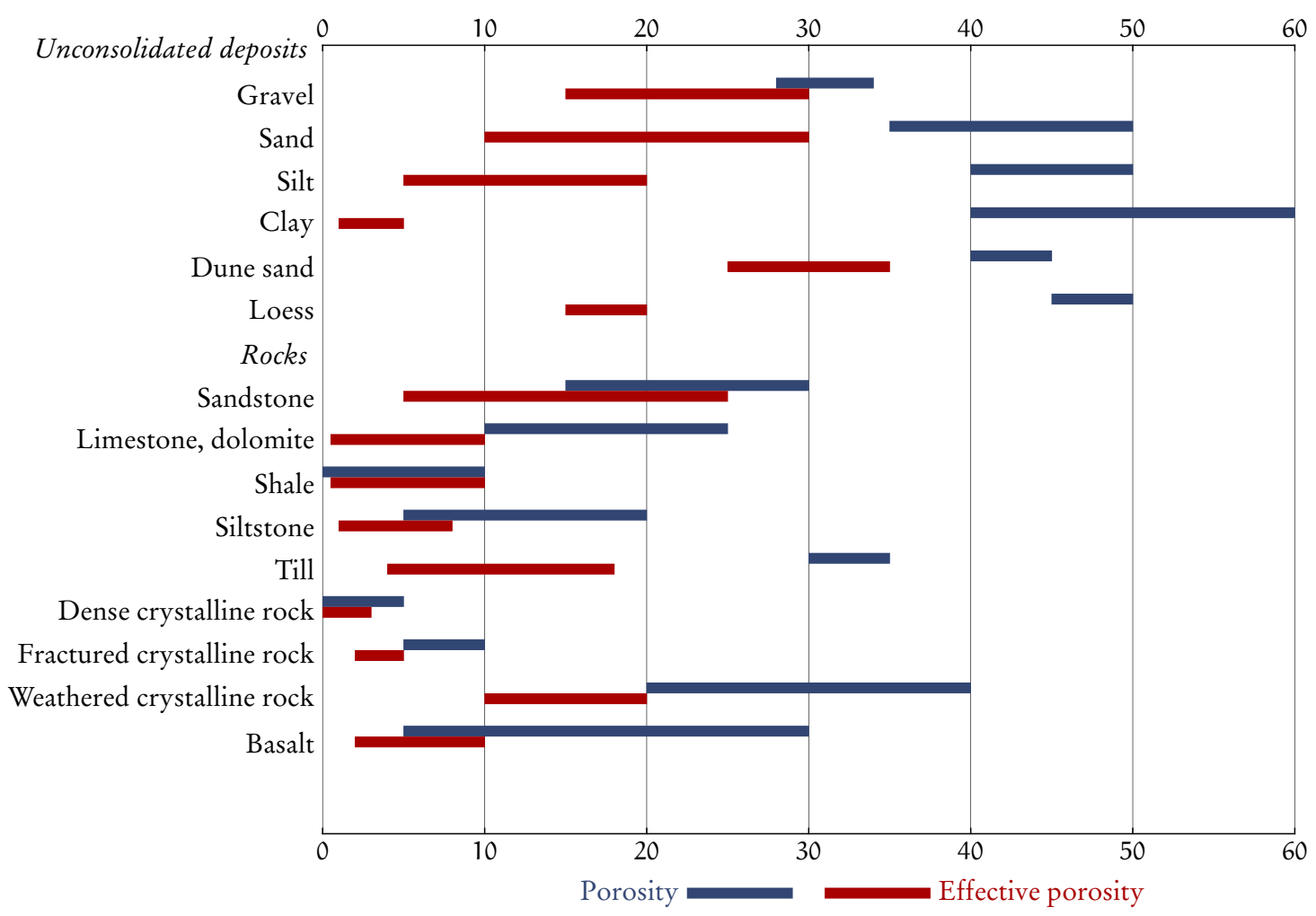

Figure 2.4.: Some porosity values of porous media (from Singhal and Gupta (1999)).

\section{I.5. POROSITY}

In section 2.I.2 we already mentioned porosity as a quantity on the macroscopic scale. Porosity is a measure of the potential storage volume for porous media. For a given sample of a porous medium, the porosity is defined as

$$
\text { porosity }=\frac{\text { pore volume }}{\text { bulk volume }}=\frac{\text { bulk volume }- \text { mineral volume }}{\text { bulk volume }}
$$




\section{The Model of Fluid Flow in Fractured Porous Media}

Porosity is a dimensionless scalar quantity. In engineering and geology, sometimes percentage values rather than fractional values are used. Porosity can be determined by visual methods and laboratory measurements, both a demanding task. Classical visual methods tend to underestimate the porosity, because small pores cannot be detected. Inaccuracies in porosity measurements arise from e.g. incomplete removal of fluids from the medium if volumetric displacement methods with strongly wetting fluids are employed, or alteration of the material: Carbonate rocks are compressible and porosity decreases with increasing effective stress. Therefore measurements should be made at in situ conditions.

Porosity is controlled by shape and arrangement of the constituent grains, the degree of sorting, compaction and cementation, fracturing and solution. Well-sorted materials have higher porosities independent of grain size, while poorly sorted materials have lower porosity because small grains occupy the pore space between larger grains.

Porosity rarely exceeds 0.45 in reality. This is understandable if we con-

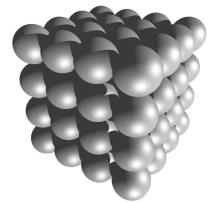

Cubic

Packed Spheres

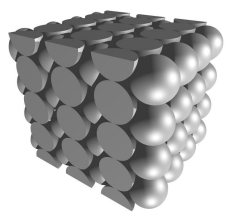

Rhombohedric Packed Spheres sider the most open type arrangement of spherical grains, the cubic packing. In this arrangement we find a porosity of $1-\Pi / 6 \approx 0.476$. The closest packing, the rhombohedral packing, has a porosity of .26 . In more realistic settings, only a fraction of the pore space may be connected. Since only the accessible pore space is important for fluid flow, two types of porosity are distinguished: The total porosity $\Phi_{t}$, which considers all void space in the porous medium, and the effective porosity $\Phi_{e}$, which includes only the interconnected pore space. Porosity is also divided into primary porosity and secondary porosity: Primary porosity is an original sedimentary feature that is present from the time of deposition. It is also called matrix porosity or intergranular porosity. The secondary porosity describes the void space that is created after deposition. One possible cause of secondary porosity is fracturing (especially in carbonates) or solution. If applicable, the term fracture porosity is used, i. e. the ratio of fracture void volume and total bulk volume.

Total porosity and effective porosity can be quite different. Figure 2.4 lists some porosity values for different materials. The values are taken from Singhal and Gupta (I999), where the effective porosity is defined as the specific yield $S_{r}$ : the ratio of volume of water that the unconfined aquifer will release from storage by gravity to the total volume of the fully saturated aquifer material. It depends on the duration of the drainage, temperature, mineral composition of the water, grain size and other textural characteristics of aquifer material.

\section{I.6. Representative Elementary Volume}

The concept of the representative elementary volume (REV) is central to the transition from the microscopic scale to the macroscopic scale. An REV is the smallest volume which is representative for the porous medium. Quantities on the microscopic scale are replaced by averaged quantities on the macroscopic scale. For these quantities, new macroscopic equations must be derived. An REv can be derived for every property of a homogeneous porous medium. We only demonstrate it here for porosity, but the derivation of the macroscopic equations is based on the existence of an REV for all properties. 


\section{I. MATERIAL PROPERTIES}
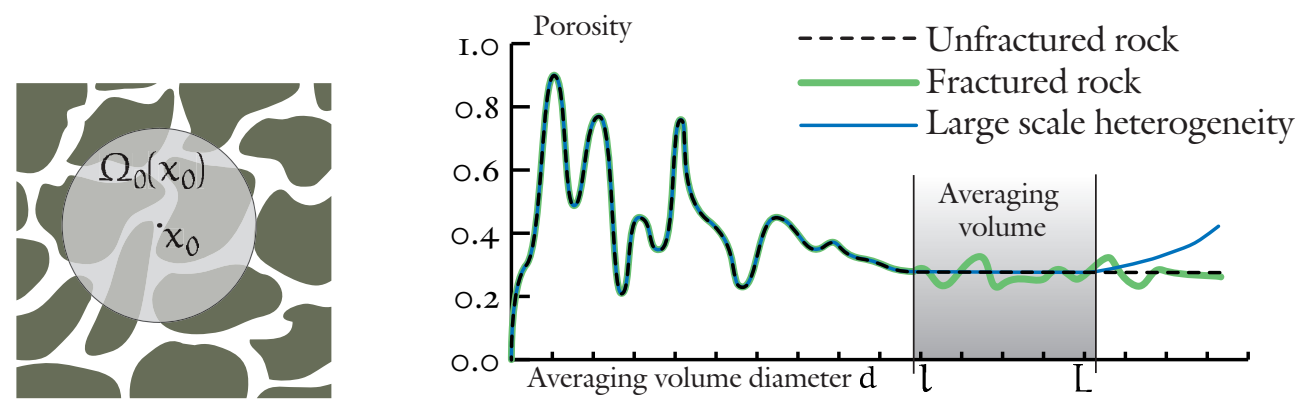

Figure 2.5.: An averaging volume $\Omega_{0}\left(x_{0}\right)$ around the point $x_{0}$ and shape of the porosity function for different materials.

The idea of an averaging volume assumes that if we consider a small subdomain of a homogeneous porous medium and measure a property in a non-destructive way and then enlarge the subdomain and measure again, that repeated measurements will (after initial fluctuations) converge to a value which is representative for the porous medium. Let us illustrate this for the porosity.

On the microscopic scale, each point $x$ of a porous medium is either part of the void space or part of the solid matrix. An averaging volume $\Omega_{0}(x)$ is a subdomain of the porous medium, centered around the point $x$ with a diameter $d$. The porosity of the porous medium with respect to the averaging volume can then be defined as

$$
\Phi(\boldsymbol{x})=\frac{1}{\left|\Omega_{0}(\boldsymbol{x})\right|} \int_{\Omega_{0}(\boldsymbol{x})} \gamma(\mathbf{y}) \mathrm{d} \mathbf{y},
$$

with the void space indicator function

$$
\gamma(x)= \begin{cases}1 & \text { if } x \in \text { void space } \\ 0 & \text { if } x \in \text { solid matrix }\end{cases}
$$

Figure 2.5 shows the averaging volume $\Omega_{0}\left(x_{0}\right)$ and three typical curves for the function $\Phi(x)$. For unfractured rock, there are averaging volume diameters $l$ and $L$, such that the porosity is constant in these bounds. If the sample consists of only a single material, $L$ may be the sample diameter. For samples with varying properties (like layers of different materials), the influence of these variations may become significant from a certain diameter $L$ on. In fractured rock, it is quite common that the porosity doesn't reach a constant value in the REV; instead the variation becomes insignificant in a certain range $\mathrm{l}<\mathrm{d}<\mathrm{L}$.

If we can determine lengths $l$ and $L$ such that for the diameter $d$ the condition $l \ll d \ll L$ holds and if the porosity does not depend on the size of the averaging volume in this range, then the averaging volume $\Omega_{0}\left(x_{0}\right)$ is called a representative elementary volume.

Determining the REV for a given medium can be difficult or even impossible, and the size of the REV can vary considerably. Fractures introduce variations into the medium and fractures appear at length scales from millimeters to kilometers, so we may ask how we can incorporate them into the averaging process. Let us consider a sample for investigation. 


\section{The Model of Fluid Flow in Fractured Porous Media}
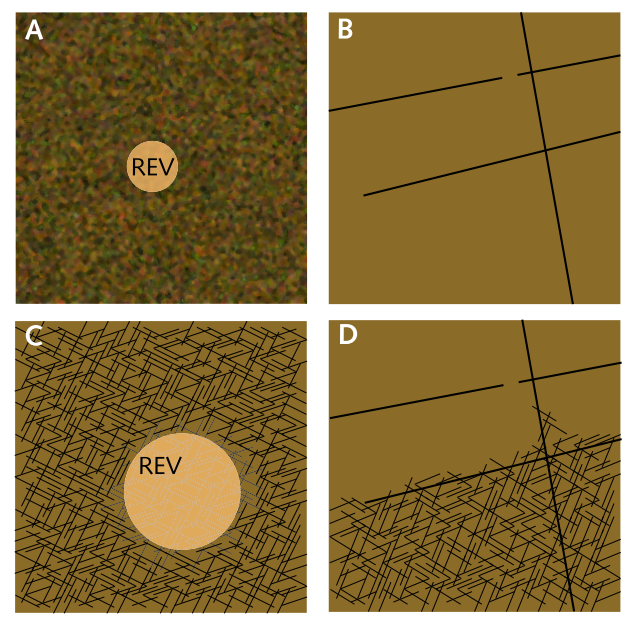

Figure 2.6.: REV size differences for fractured media.

Figure 2.6 show the four possible settings we can distinguish. If the sample shows no fracturing (case A), the averaging process can take place as described above and the REV will be comparably small. In case B some large scale fractures are present, but the remaining material is unfractured. Since the REV size easily fits into the space between the fractures, we can find an REV for the matrix and can determine a separate REV for the fractures. (Singhal and Gupta (1999) stress the point that finding an REV for a fracture is in general more difficult than finding a rock matrix REV.) If the fractures occur on a smaller scale (microfractures in case C), i.e. if every averaging volume intersects with one or more fractures before the REV size that we can expect from the unfractured material is reached, then we must use a much larger averaging volume which incorporates so many fractures that an averaging is possible. Combinations of both situations A, B and C are possible; this is case D, where three different REVS are necessary: for the unfractured matrix, for the fractured matrix and for the large scale fractures.

\section{I.7. Heterogeneity ANd ANisotropy}

If a macroscopic quantity of a porous medium is independent of its position, the porous medium is said to be homogeneous with respect to this quantity. Otherwise it is called heterogeneous. Natural soils are usually not homogeneous.

A porous medium is isotropic w.r.t. a tensorial quantity if this quantity is independent of direction; otherwise it is called anisotropic. Anisotropy can be introduced by anisotropic shape and arrangement of the grains. Figure 2.7 shows (a) the pore scale of an isotropic and homogeneous medium and (b) the pore space of an anisotropic medium. Due to shape and arrangement of the grains, fluids will flow preferentially in horizontal direction as opposed to flow in vertical direction in case (b). Anisotropy and heterogeneity can also be introduced by fractures. The porous medium (c) of figure 2.7 shows a fractured medium with two 

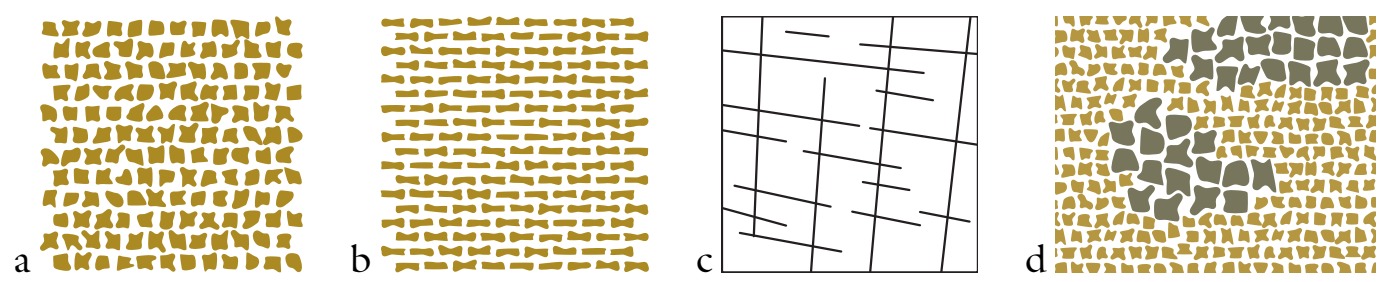

Figure 2.7.: An isotropic porous medium (a), anisotropic media due to pore anisotropy (b) and fractures (c), and a heterogeneous medium (d).

main fracture directions. These directions dominate fluid flow and transport. Medium (d) contains domains of different grain sizes and is therefore heterogeneous.

\subsection{Single PHASE FLUID FLOW IN FRACTURED POROUS MEDIA}

In contrast to the vast amount of competing theories considering different aspects in fluid flow in the subsurface, we are in the fortunate situation that there is only a single approach for the macroscopic momentum equation. The valid equation is by common consent of the porous media community the famous Darcy's law. It was found I856 experimentally by the french engineer Henry Darcy, who investigated the flow of water in vertical homogeneous sand filters Darcy (I856).

We present Darcy's law and then consider under what circumstances Darcy's law is applicable in a single fracture. The application to interacting matrix-fracture networks is done in section 2.5.

\subsection{DARCY'S LAW FOR FLOW OF A SINGLE FLUID PHASE IN A POROUS MEDIUM}

Darcy's law states that for the averaged velocity $\boldsymbol{u}$ the equation

$$
\mathbf{u}=-\mathrm{K}_{0} \nabla \mathrm{h}
$$

holds, with the tensor of bydraulic conductivity $\mathrm{K}_{0}$ and the piezometric head $\mathrm{h}$,

$$
\mathrm{h}=\frac{\mathrm{p}}{\rho \mathrm{g}}+z .
$$

$\mathrm{p} / \rho \mathrm{g}$ is the pressure head and $z$ is the elevation head. The tensor of hydraulic conductivity can be split into

$$
\mathrm{K}_{0}=\mathrm{K} \frac{\rho g}{\mu} .
$$

The quantities introduced in these equations and their units are:

$\mathbf{u}(\boldsymbol{x}, \mathrm{t}) \quad$ The Darcy velocity, also called macroscopic apparent velocity. This is the velocity which is observable at the macroscale, while the velocity in the pore channels is $\mathbf{u} / \Phi$.

$[\mathrm{m} / \mathrm{s}]$ 


\section{The Model of Fluid Flow in Fractured Porous Media}

$p(x, t) \quad$ The fluid pressure.

$[\mathrm{Pa}]=\left[\mathrm{N} / \mathrm{m}^{2}\right]$

$\rho(x, t) \quad$ The fluid density. It is either assumed to be constant for incompressible fluids or we employ as the equation of state the ideal gas law $p=\rho R T$ with the temperature $T$ and an individual gas constant $R$.

$\left[\mathrm{kg} / \mathrm{m}^{3}\right]$

$\mu(\boldsymbol{x}, \mathrm{t}) \quad$ The dynamic viscosity of the fluid. We consider only applications where $\mu$ is constant.

$[\mathrm{Pas}]=[\mathrm{kg} /(\mathrm{ms})]$

g The gravity vector. It points in the direction of gravity (the $z$-direction unless stated otherwise) with size $g, \mathbf{g}=(0,0,-g)^{\top}=(0,0,-9.81)^{\top}$ on middle latitudes of the earth.

$\left[\mathrm{m} / \mathrm{s}^{2}\right]$.

$\mathrm{K}(\boldsymbol{x}) \quad$ The symmetric tensor of absolute permeability or intrinsic permeability. It depends only on position (for heterogeneous media), not on the fluid, and may introduce anisotropy.

The form of Darcy's law that we will use is

$$
\mathbf{u}=-\frac{K}{\mu}(\nabla p-\rho \mathbf{g}) .
$$

Despite the consent on the validity of Darcy's law, the proper assumptions for the derivation of Darcy's law from the Navier-Stokes equations are still investigated. We state the incomplete set of assumptions that the flow is laminar (so that inertial effects can be neglected), that the fluid is assumed to be Newtonian, and that a no-slip boundary condition is valid at the microscopic scale at fluid-solid interfaced. For details we refer to Hornung (1997) and Bear (1972).

Equation (2.6) is completed by the equation for macroscopic fluid mass conservation. Let the domain defined by the porous medium under consideration be $\Omega \subset \mathbb{R}^{3}$. Then

$$
\frac{\partial(\Phi \rho)}{\partial t}+\nabla \cdot(\rho \mathbf{u})=\rho q \quad \text { in } \Omega .
$$

Here we employ the porosity $\Phi$ and source and sink terms q.

$\Phi \quad$ The porosity as defined in section 2.I.5. We use the effective porosity, since this defines the volume available for fluid flow. Although not considered in this context, the porosity may depend on the fluid pressure $p$, time and temperature, like in the swelling of clay or in freezing soils (see Ippisch (200I)).

$\mathrm{q}(x, \mathrm{t}) \quad$ Source and sink term.

Inserting equation 2.6 into equation 2.7 yields

$$
\frac{\partial(\Phi \rho)}{\partial t}-\nabla \cdot\left(\rho \frac{K}{\mu}(\nabla p-\rho \mathbf{g})\right)=\rho q \quad \text { in } \Omega .
$$

This equation for the unknown $p$ is completed by appropriate initial conditions and boundary conditions at the Dirichlet boundary $\Gamma_{\mathrm{D}}$ and the Neumann boundary $\Gamma_{\mathrm{N}}, \partial \Omega=\Gamma=$ $\Gamma_{\mathrm{D}} \cup \Gamma_{\mathrm{N}}$ :

$$
p(x, 0)=p_{0}(x) \quad \text { in } \Omega,
$$




$$
\begin{aligned}
& p(x, t)=p_{D}(x, t) \quad \text { on } \Gamma_{D}, \\
& \rho \mathbf{u} \cdot \mathfrak{n}=\phi(x, t) \quad \text { on } \Gamma_{N} \text {. }
\end{aligned}
$$

If the fluid is incompressible, $\rho$ is constant and (2.8) reduces to an elliptic equation; if the fluid is compressible, the equation is of parabolic type.

The assumption that only laminar flow takes place (where viscous forces are dominant) is too restrictive in some situations. Especially in fractures with high hydrodynamic conductivity fluid flow can be very fast. According to Sahimi (1995), inertial forces become important for Reynolds numbers Re $>10$ if we choose the characteristic length of the Reynolds number to $\sqrt{\mathrm{K}}$ or $\sqrt{\mathrm{K} / \Phi}$; other authors suggest the limit for Re between 1 and 10 (Bear, 1972). For $\operatorname{Re}>100$ turbulence occurs. Then Darcy's law is no longer valid, as well as for the transition zone between these limits. Alternatives to Darcy's law were proposed by Forchheimer (I9OI) and Brinkman (I947).

\subsubsection{Single PHASE FLUID FLOW IN A FRACTURE}

Fluid flow in a filled fracture can be treated by Darcy's law, since a filled fracture is a porous medium. For open fractures, a different situation arises, which has been addressed by many researchers.

A very idealized approximation of an open fracture is the parallel plate model, also called Hele-Shaw analog. We will comment on the numerous limitations of this model later, but include it despite its flaws since it is the starting point for many models of fluid flow in a fracture. Assuming a setup of two parallel plates arranged at a distance $b$, we find that the averaged velocity between the plates is

$$
\mathbf{u}=\frac{\mathrm{b}^{2}}{12} \frac{\rho g}{\mu} \nabla h
$$

This means that Darcy's law is valid in the opening if we choose $\mathrm{K}=\mathrm{b}^{2} / 12$.

This relation is often called the local cubic law, because the volumetric flux according to equation 2.12 is

$$
\mathrm{Q}=\frac{\mathrm{b}^{3}}{12} \frac{\rho \mathrm{g}}{\mu} \nabla \mathrm{h},
$$

where the flux $\mathrm{Q}$ is proportional to the third power of the fracture width $\mathrm{b}$.

Appendix A shows how equation 2.I2 can be derived from the Navier-Stokes equations. An experimental validation of the cubic law was given by Witherspoon et al. (I980).

In Singhal and Gupta (I999) several extensions to the parallel plate model are listed. They account for nonparallel flow, turbulent flow, permeable plate walls and for wall roughness. For parallel flow with mostly smooth walls, turbulence occurs for Reynolds numbers Re $>$ 2400 according to Lomize (I95I), Romm (1966) or Re $>2300$ according to Louis (I969). For rough wall surfaces, turbulence may start as early as $\operatorname{Re}>300$.

The cubic dependence on the fracture width can quite easily dominate other factors which influence fluid flow. This is an important consideration for laboratory experiments with 


\section{The Model of Fluid Flow in Fractured Porous Media}

rock material from great depths, because the fracture width can change considerably in the absence of the large pressures.

A review of open questions concerning fluid flow in fractures and fractured media can be found in Berkowitz (2002). From the long list of current research fields we mention the effect of channeling (Neretnieks et al., I982, Neretnieks, I993, Smith and Schwartz, 1984, Tsang and Tsang, 1987), which describes the development of channels along which the majority of fluid flow and transport processes takes place. Tsang and Neretnieks (I998) contains an overview of experiments on different scales which show the importance of this effect. Another important issue is the the modeling of transport behavior at fracture intersections (Park et al., 2001, Adler and Thovert, 1999, Mourzenko et al., 2002).

\subsection{Multiphase flow at the miCRoscopic sCAle}

The flow of a single fluid phase is driven by pressure forces due to pressure differences and gravitational forces only. If two or more phases are present in the system, a new force is introduced, the capillary force at the interface between the fluid phases. The capillary force has a significant influence on the fluid behavior. We will consider the microscale to investigate the origin and behavior of the capillary force and will then move to a continuum model on the macro scale in the next section.

\subsection{CAPILLARY FORCES AND THE SHAPE OF THE CAPILLARY SURFACE}

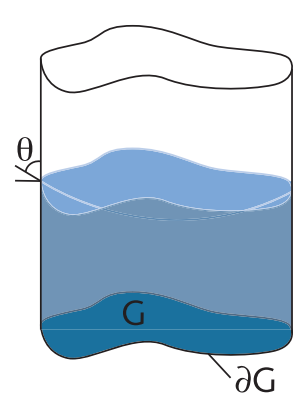

To explain the origin of the capillary force we must retract to the the microscopic scale. If we consider a capillary tube with gas and water, there is an interfacial tension caused by the cohesive forces which attract molecules in a fluid to each other and adhesive forces between the solid matrix and the fluid phases. The interaction of these forces leads to a specific angle $\theta$ between the solid matrix and the interface between the phases. This contact angle depends on the fluids. It also depends on temperature and chemical constituents of the fluids, but these dependencies are neglected here.

If we want to determine the shape of the interface between two phases in a container, we must take into account the adhesive force at the wall and the capillary force at the free boundary which develops because fluid molecules are not surrounded by other fluid molecules. Following Zeidler (I995) the equation for the free surface is

$$
z=u(x, y) \text { in } \mathrm{G}
$$

and $\mathrm{u}$ can be determined by the variational problem

$$
\underbrace{\sigma \int_{G} \sqrt{1+u_{x}^{2}+u_{y}^{2}} d x d y}_{\text {energy stored by deformation }}-\underbrace{\sigma \beta \int_{\partial G} u d s}_{\text {work by adhesion }}-\underbrace{\int_{G} \rho g u d x d y}_{\text {work by gravitation }}=\min !
$$


with the side condition

$$
\int_{G} u d x d y=V
$$

$\sigma$ is the surface tension, $\rho$ is the density of the fluid, $\beta$ is the relative adhesion coefficient, $\mathrm{g}$ is the gravitational acceleration and $\mathrm{V}$ is the volume of the fluid. We assume that the surface energy is proportional to the surface area and that the adhesion forces at the wall are proportional to the area of the wall wetted by the fluid. Then it is possible to prove that with

$$
\mathrm{T}:=\frac{\nabla \mathrm{u}}{|\nabla \mathrm{u}|}
$$

the variational problem satisfies

$$
\begin{aligned}
\operatorname{div} T & =\kappa u+\lambda & & \text { in } G, \\
\operatorname{Tn} & =\beta & & \text { on } \partial \mathrm{G} .
\end{aligned}
$$

$\boldsymbol{n}$ is the outward unit normal of $\partial G, k=g \rho / \sigma$ is the capillary constant and

$$
\lambda=\beta \frac{\operatorname{length}(\partial G)}{\operatorname{meas}(G)}-\kappa \frac{V}{\operatorname{meas}(G)} \text { and } \cos \theta=\beta .
$$

Equation 2.18a can be written as

$$
\frac{\partial}{\partial x}\left(\frac{u_{x}}{\sqrt{1+u_{x}^{2}+u_{y}^{2}}}\right)+\frac{\partial}{\partial y}\left(\frac{u_{y}}{\sqrt{1+u_{x}^{2}+u_{y}^{2}}}\right)=\kappa u+\lambda .
$$

The outer unit vector to the free surface is

$$
N=\frac{e_{z}-u_{x} e_{x}-u_{y} e_{y}}{\sqrt{1+u_{x}^{2}+u_{y}^{2}}}
$$

with the Cartesian basis $\left(\mathbf{e}_{x}, \mathbf{e}_{y}, \mathbf{e}_{z}\right)$. The cosine of the boundary angle $\theta$ is defined as

$$
\cos \theta=-\mathbf{n N}=\mathrm{Tn}
$$

which implies $\cos \theta=\beta$ by the boundary condition 2.I6).

The differential equation 2.I5) (without gravity) dates back to papers by Young in I805 and Laplace in I806. The heuristic arguments in these works are still employed in the engineering literature. A rigorous approach was proposed by Gauss in 1830 , based on the Lagrange multiplier rule. Note that this derivation describes the static situation. If the fluids are moving due to some external driving force, the contact angle is subject to change, as will be discussed in section 2.3.4.

The fluid with $\theta<90^{\circ}$ is called the wetting fluid because of its tendency to adhere to the solid and spread over it. The other fluid is called the nonwetting fluid. The wettability 


\section{The Model of Fluid Flow in Fractured Porous Media}

generally decreases from water over the non-aqueous phase liquid (NAPL) to gas, with water being the wetting fluid w.r.t. NAPL and gas, and NAPL being the intermediate wetting phase if we have a three-phase system.

\subsubsection{CApillary pressure}

Across the interface between the wetting phase and the nonwetting phase a jump discontinuity occurs in the pressure, because the pressure $p_{n}$ in the nonwetting phase is larger than the pressure $p_{w}$ in the wetting phase. This jump is termed capillary pressure $p_{c}$,

$$
p_{c}:=p_{n}-p_{w} \geq 0 .
$$

For the idealized situation of two phases in a tube with a small radius $r$ (so that we can neglect gravitational force) we find that

$$
p_{c}=\frac{2 \sigma \cos \theta}{r}
$$

and the capillary surface has constant mean curvature. $\sigma$ is the surface tension, the ratio between the amount of work $\Delta W$ necessary to enlarge the area of the surface by $\Delta A$, and has the unit $\left[\mathrm{J} / \mathrm{m}^{2}\right]$.

For more general geometries the Laplace equation describes the capillary pressure as

$$
p_{c}=\sigma\left(\frac{1}{r_{1}}+\frac{1}{r_{2}}\right)
$$

with the mean radii of curvature $r_{1}$ and $r_{2}$.

These equations allow for a prediction of fluid behavior in the pore space.

\subsubsection{CAPIllary BeHAVIOR IN THE PORE SPACE AND IN FRACTURES}

From the capillary pressure equations $(2.23)$ and $(2.24)$ we find that for smaller capillary tubes the capillary pressure increases and that for increasing radii of the tube the capillary pressure decreases. The consequence for a porous medium is that if the porous medium is not fully water-saturated, the water will retreat from the largest pores and reside in the small pores of the medium and that large pores of the medium will only be filled when more water is present. The static situation is described in figure 2.8; on the left only a small amount of water is present in the volume and the water is in the small pores and it is spread as a thin film over the grains. Capillary pressure is high, and little water flow can take place because the water phase is largely disconnected. The open fracture can be considered as a large pore. Consequently it contains no water. It may strike as counterintuitive, but fractures act as barriers for water flow in this situation. If large amounts of water are present, the nonwetting phase fills only the largest pores, as shown on the right of figure 2.8. The capillary pressure is small and water flow can easily take place, especially in the fractures.

The same considerations are in principle valid for flow in a single fracture, too. However, the processes in a single fracture are complex and not yet fully understood. A discussion 

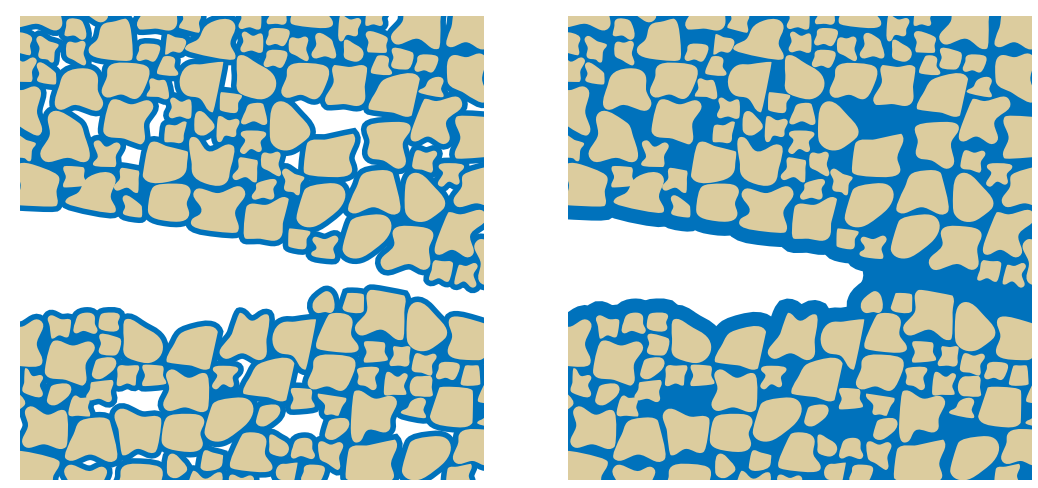

Figure 2.8.: Distribution of the wetting phase for high capillary pressure and low capillary pressure.
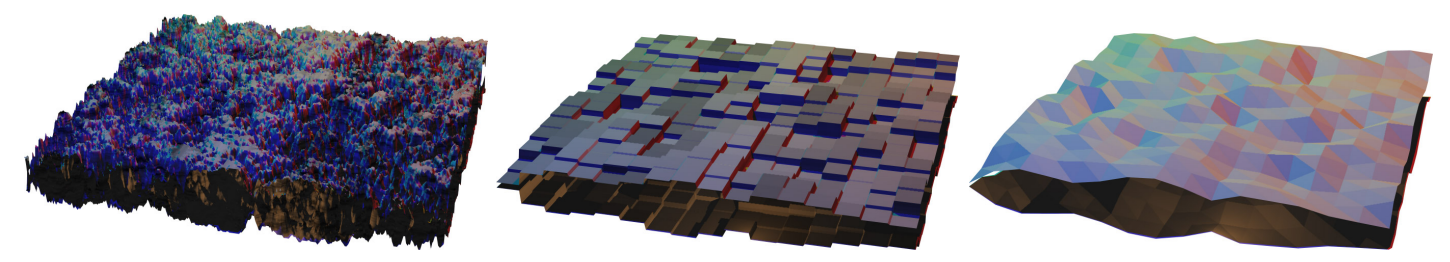

Figure 2.9.: Parallel plate and sawtooth approximation of fracture walls.

of all issues concerning this problem is beyond the scope of this work; Berkowitz (2002) explains some of the difficulties.

One large complexity is introduced by fracture wall roughness. The parallel plate model starts by approximating fracture walls by regions of parallel plates with varying widths, see figure 2.9. For parallel plates the capillary pressure is

$$
p_{c}=\frac{4 \sigma \cos \theta}{b}, \quad \text { and } \quad b_{c}:=\frac{4 \sigma \cos \theta}{p_{c}}
$$

is the cut-off fracture aperture which indicates if (for fixed $p_{c}$ ) the wetting phase is present $\left(b \leq b_{c}\right)$ or if the nonwetting is present $\left(b>b_{c}\right)$.

Recent results have shown, that this model is too simple and that wetting phase can also be present in regions of the fracture with greater width; see Jakobs (2003) and the literature cited therein.

\subsubsection{Dynamic processes at the microscale}

So far we only considered equilibrium situations. If water-saturated soil is drained (drainage) or if the soil is being filled with water (imbibition), the processes are more complex. These dynamic processes introduce hysteresis into the system: For an accurate description we need to take the history of a porous medium into account. The question whether we are in a drainage or imbibition process is important and has a significant influence on the flow. 


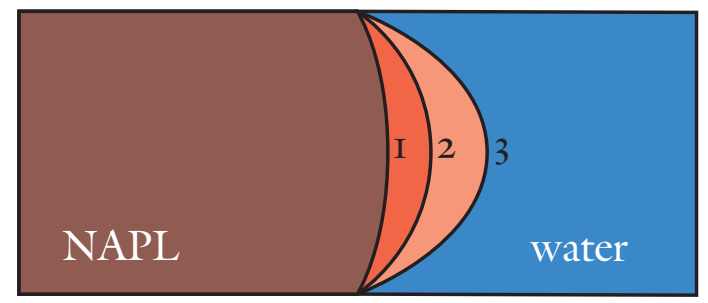

I) Displacement of NAPL by wetting phase

2) Stationary state

3) Displacement of wetting phase by NAPL

Figure 2.IO.: Contact angle hysteresis. The contact angle depends on the flow direction.

We will only consider processes in one direction, i. e. only drainage or imbibition, and we assume that appropriate parameters for this process are known. If, however, we would like to explore processes where drainage and imbibition alternate, hysteresis would become important and our model would have to be extended.

Contact angle hysteresis Depending on the direction of displacement, the contact angle and the capillary pressure change. This is called contact angle hysteresis. Figure 2.IO shows the shape of the capillary surface for drainage, equilibrium and imbibition.

INK BOTTLE EFFECT Due to the variability of the pore channel width, the fluid volume can be different for the same capillary pressure, depending on whether drainage or imbibition takes place.

Residual SATURATion The displacement of one fluid phase by another may not result in complete removal of the displaced phase from the pore space. During drainage, the wetting phase retracts to smaller pores until a situation similar to the one on the left in figure 2.8 is reached. The remaining wetting phase volume can not be displaced by advective processes and is called residual saturation (a rigorous definition is given in section 2.4). Phase transitions (like vaporization) can reduce the water volume below the residual saturation; however, phase transitions are not considered in this work. For the treatment of phase transitions see Ippisch (200I), Helmig (1997), Class (2001).

The similar phenomenon can be observed during imbibition where the nonwetting phase can become entrapped in large pores of the porous medium.

ENTRY PRESSURE If a porous medium is water-filled, then a certain pressure must be reached in the nonwetting phase before it can penetrate the sample. This is called the entry pressure, sometimes also threshold pressure or bubbling pressure. Consequently the capillary pressure must reach a certain level before displacement can take place. This effect is also important at media continuities and will be discussed later. 


\subsection{The transition to the Macroscale}

\subsection{The TRANSITION TO THE MACROSCALE}

The sharp separation between phases at the microscopic scale does not exist on the macroscopic scale. On this scale, we employ a continuum model through averaging like in the case of the Darcy law for a single fluid phase.

Let us consider two fluid phases in the void space of a porous medium ${ }^{2}$. The phases are denoted by lower index $w$ for the wetting phase and lower index $n$ for the nonwetting phase. If we consider water-gas systems, we sometimes use lower index $g$ to indicate the gas phase. For general phases we use greek symbols $\alpha, \beta, \gamma, \ldots$

\subsection{SATURATION}

The saturation of a fluid phase $\alpha$ in the void space of an REV is

$$
S_{\alpha}:=\frac{\text { volume of fluid within the REV }}{\text { volume of voids in the REV }} .
$$

This can also be expressed by employing a phase indicator function for each point $x$ in the porous media domain $\Omega$ (after Gray and Lee (1977)),

$$
\gamma_{\alpha}(x, t):= \begin{cases}1 & x \in \text { phase } \alpha \text { at time } t \\ 0 & \text { else. }\end{cases}
$$

The saturation in an REV $\Omega_{0}\left(x_{0}\right)$ around $x_{0}$ is then

$$
S_{\alpha}\left(x_{0}, t\right):=\int_{\Omega_{0}\left(x_{0}\right)} \gamma_{\alpha}(x, t) d x / \int_{\Omega_{0}\left(x_{0}\right)} \gamma(x) d x
$$

with the void space indicator function $\gamma$ from equation (2.2). Note the dependence on time $t$ for $\gamma_{\alpha}(x, t)$ and $S_{\alpha}(x, t)$.

The phases fill the void space,

$$
\sum_{\alpha} S_{\alpha}(x, t)=1
$$

and this implies

$$
S_{n}=1-S_{w} \quad \text { and } \quad S_{w}=1-S_{n}
$$

in the case of two phases $w$ and $n$.

\footnotetext{
${ }^{2}$ The discussion of more than two fluid phases can be found in Helmig (I997).
} 


\section{The Model of Fluid Flow in Fractured Porous Media}

\subsubsection{Multiphase FLOW EQUATIONS}

For a multiphase system in a porous medium which fills the domain $\Omega \subset \mathbb{R}^{3}$ we require conservation of mass for each phase $\alpha$ separately:

$$
\frac{\partial\left(\Phi \rho_{\alpha} S_{\alpha}\right)}{\partial t}+\nabla \cdot\left(\rho_{\alpha} \mathbf{u}_{\alpha}\right)=\rho_{\alpha} \mathbf{q}_{\alpha}
$$

$\rho_{\alpha}$ is the density of phase $\alpha, q_{\alpha}$ is the source term of phase $\alpha$ and $\boldsymbol{u}_{\alpha}$ is the Darcy velocity of phase $\alpha$.

Under the assumption that momentum transfer between phases is negligible it can be shown by homogenization or volume averaging techniques (see Hornung (I997)) that an extension of Darcy's law holds for each phase,

$$
\mathbf{u}_{\alpha}=-\frac{K_{\alpha}}{\mu_{\alpha}}\left(\nabla p_{\alpha}-\rho_{\alpha} \mathbf{g}\right)
$$

The Darcy velocity now only depends on the macroscopic phase pressure $p_{\alpha}$.

In the presence of two fluids the movement of one fluid interferes with the other, so we can expect the phase permeability $\mathrm{K}_{\alpha}$ to be smaller than the absolute permeability $\mathrm{K}$, since only a smaller portion of the void space is available for fluid flow. This requires that the phase permeability depends on the saturation. The common technique to introduce this dependence into the model is by the definition of the relative permeability $k_{r \alpha}$. The relative permeability is used together with the absolute permeability to yield the phase permeability $\mathrm{K}_{\alpha}$ :

$$
\mathrm{K}_{\alpha}=\mathrm{k}_{\mathrm{r} \alpha}\left(\mathrm{S}_{\alpha}\right) \mathrm{K} .
$$

The relative permeability is a dimensionless factor. Functions for $k_{r \alpha}\left(S_{\alpha}\right)$ are given below.

\subsubsection{CApillary PRESSURE Functions}

The capillary pressure is also part of the macroscopic description of multiphase flow and is defined as

$$
p_{c}\left(S_{w}\right)=p_{n}-p_{w}
$$

for the pressure of the wetting phase $p_{w}$ and the nonwetting phase $p_{w}$. From the microscopic considerations we expect that the capillary pressure is small when the porous medium is fully saturated with water. During drainage, capillary pressure increases as the water retreats to the smaller pores, until all water is removed from the the pore space or until the residual saturation $S_{w r}$ is reached. Here we neglect the influence of temperature and fluid composition on the capillary pressure. We expect $p_{c}$ to be a monotonically decreasing function which depends solely on the saturation of the wetting phase $S_{w}$. Of course we could make it also depend on $S_{n}$, since we know $S_{w}=1-S_{n}$ from equation 2.29.

The shape of the function depends on the pore space geometry. For very well graded materials, we observe almost linear behavior of the function, while for poorly graded media 


\subsection{The transition to the macroscale}

a strong nonlinearity develops. In figure 2.II, the curves for $n=2$ (left) and $\lambda=1 / 2$ (right) correspond to well sorted materials while the curves for $n=5$ (left) and $\lambda=4$ (right) correspond to poorly sorted material.

Two capillary pressure functions are most commonly used, which both employ the effective saturation $\mathrm{S}_{e}$ of the wetting phase,

$$
S_{e}:=\frac{S_{w}-S_{w r}}{1-S_{w r}} \quad S_{w r} \leq S_{w} \leq 1
$$

The effective saturation can also be defined for each phase $\alpha$ as

$$
S_{e \alpha}:=\frac{S_{\alpha}-S_{\alpha r}}{1-S_{w r}-S_{n r}} \quad S_{w r} \leq S_{w} \leq 1-S_{r n} .
$$

The decision, if $S_{e}$ or $S_{e \alpha}$ is appropriate depends on the processes which take place in the porous medium. See Helmig (1997, pp. 66) for a discussion of this matter. We employ 2.34 throughout this work.

The first function is the Van Genuchten capillary pressure function, which was derived for water-gas systems:

$$
p_{c}\left(S_{w}\right)=\frac{1}{\alpha}\left(S_{e}^{-\frac{1}{m}}-1\right)^{\frac{1}{n}}
$$

with parameters $\alpha, n$ and $m$ which have to be fitted to the data. $m$ is usually chosen as $m=1-1 / n . \alpha$ is related to the entry pressure.

The Brooks-Corey capillary pressure function is

$$
p_{c}\left(S_{w}\right)=p_{d} S_{e}^{-\frac{1}{\lambda}}
$$

$p_{d}$ is the entry pressure, and $\lambda$ is related to the pore size distribution: Materials with small variations in pore size have a large $\lambda$ value while materials with very different pore sizes have small $\lambda$ values. Usually $\lambda$ is in the range $0.2 \leq \lambda \leq 4$.

Figure 2.II shows some examples of capillary pressure functions. Different correlations between the Brooks-Corey capillary pressure function and the Van Genuchten capillary pressure functions for equal physical conditions are compared in Ma et al. (1999). They found the correlation presented by Lenhard et al. (1989) to be the most accurate:

$$
\begin{aligned}
\lambda & =(n-1)\left(1-(1 / 2)^{\frac{n}{n-1}}\right) \\
p_{d} & =\frac{1}{\alpha}\left(0.72-0.35 e^{-n^{4}}\right)^{\frac{1}{\lambda}}\left(\left(0.72-0.35 e^{-n^{4}}\right)^{\frac{n}{1-n}}-1\right)^{\frac{1}{n}}
\end{aligned}
$$

The models of Brooks-Corey and van Genuchten were developed for porous media and we assume that a similar capillary pressure function exists also for fractures, either filled or open. However, the capillary pressure in a single open fracture was experimentally investigated in Bertels et al. (200I) by means of computed tomography (CT) scanning to measure aperture distribution and saturation along with capillary pressure and relative permeability. 


\section{The Model of Fluid Flow in Fractured Porous Media}
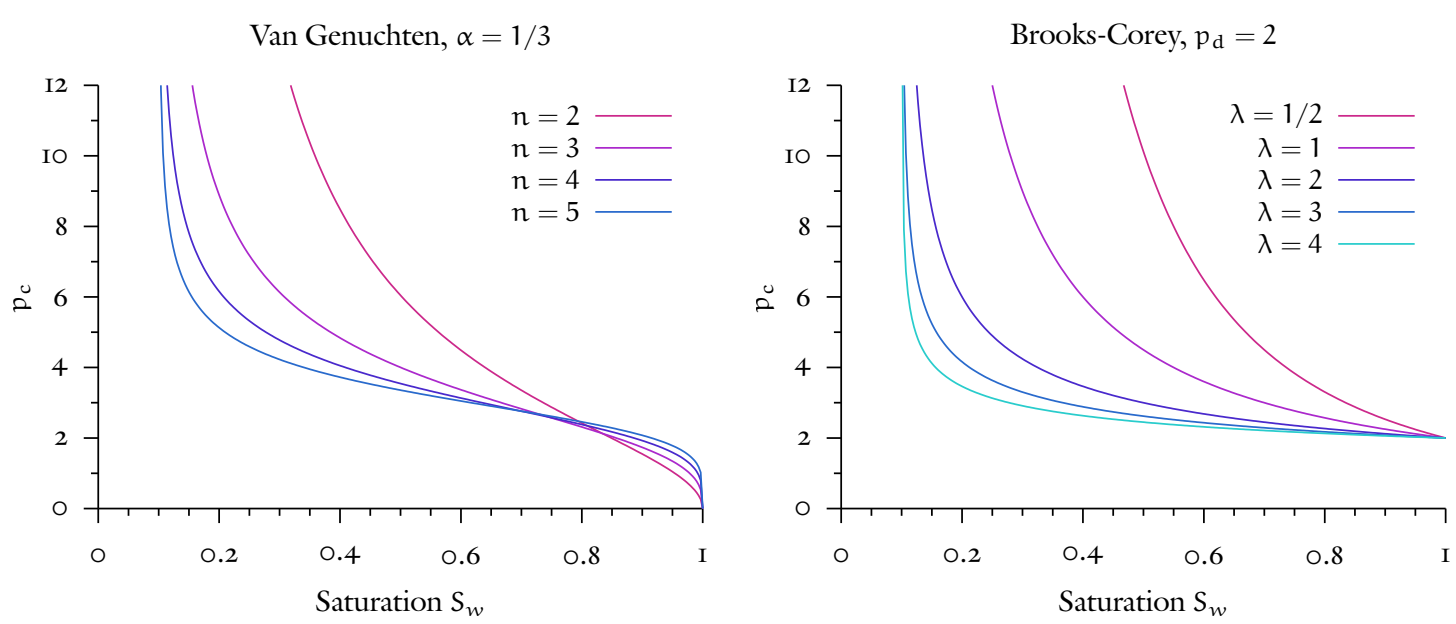

Figure 2.II.: Capillary pressure functions after Van Genuchten and Brooks-Corey

They found that the capillary pressure curve shows non-monotonic behavior, contrary to the above model. After an initial increase of capillary pressure with increased gas saturation, the capillary pressure decreases with increasing gas saturation, probably due to local rearrangement of fluid under the influence of both capillary and viscous forces. This indicates that a macroscopic description of capillary pressure in open fractures may be inadequate.

\subsubsection{RELATIVE PERMEABILITY FUNCTIONS}

For the relative permeability $k_{r \alpha}$ we present the two models that describe $k_{r \alpha}$ as a function of $S_{\alpha}$ which are most commonly used. The Van Genuchten relative permeability functions are

$$
\begin{aligned}
& k_{r w}=S_{w e}^{\epsilon}\left(1-\left(1-S_{w e}^{\frac{n}{n-1}}\right)^{\frac{n-1}{n}}\right)^{2}, \\
& k_{r n}=S_{n e}^{\gamma}\left(1-\left(1-S_{n e}\right)^{\frac{n}{n-1}}\right)^{\frac{2(n-1)}{n}} .
\end{aligned}
$$

with parameters $\epsilon$ and $\gamma$ which are usually chosen $\epsilon=1 / 2$ and $\gamma=1 / 3$.

The relative permeability functions after Brooks-Corey are

$$
\begin{aligned}
& k_{\mathrm{rw}}\left(S_{w}\right)=S_{w e}^{\frac{2+3 \lambda}{\lambda}} \\
& k_{\mathrm{rn}}\left(S_{w}\right)=S_{n e}^{2}\left(1-\left(1-S_{n e}\right)^{\frac{2+\lambda}{\lambda}}\right)=\left(1-S_{e}\right)^{2}\left(1-S_{e}^{\frac{2+\lambda}{\lambda}}\right) .
\end{aligned}
$$

The function $k_{r w}\left(S_{w}\right)$ increases only slowly for small saturations, because the wetting phase fills the smallest pores first where fluid flow is hindered by the strong molecular adhesion forces. Only for large values of $S_{w}$ the large pores will be filled and enable easier flow, i. e. a larger $k_{r w}$ value.

For the nonwetting fluid the increase of $k_{r n}\left(S_{n}\right)$ for small $S_{n}$ is faster because the fluid fills the largest pores first where the fluid can flow easily. For increasing $S_{n}$ values the nonwetting 


\subsection{The transition to the macroscale}
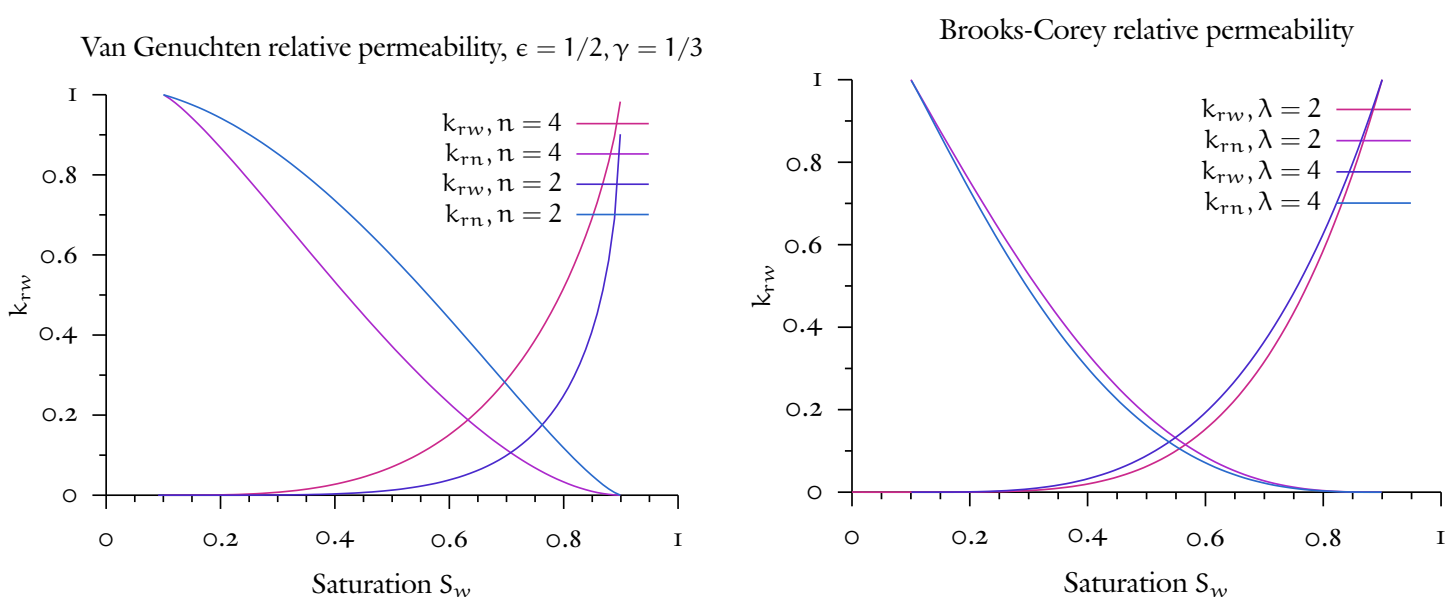

Figure 2.I2.: Relative permeability functions after Van Genuchten and Brooks-Corey

fluid phase fills also the smaller pores which contribute less to the overall flux rate, hence the smaller increase in the function.

\subsubsection{THE MODEL FOR TWO-PHASE FLOW IN POROUS MEDIA}

The model for the flow of a wetting fluid phase $w$ and a nonwetting fluid phase $n$ in a porous medium which fills the domain $\Omega \subset \mathbb{R}^{3}$ is

$$
\begin{aligned}
\frac{\partial \Phi \rho_{w} S_{w}}{\partial t}+\nabla \cdot\left(\rho_{w} \mathbf{u}_{w}\right) & =\rho_{w} \mathbf{q}_{w} \quad \text { in } \Omega, \\
\frac{\partial \Phi \rho_{\mathrm{n}} S_{\mathfrak{n}}}{\partial t}+\nabla \cdot\left(\rho_{\mathfrak{n}} \mathbf{u}_{\mathfrak{n}}\right) & =\rho_{\mathrm{n}} \mathbf{q}_{\mathfrak{n}} \quad \text { in } \Omega, \\
\mathbf{u}_{w} & =-\frac{k_{r w}}{\mu_{w}} K\left(\nabla p_{w}-\rho_{w} \mathbf{g}\right), \\
\mathbf{u}_{\mathfrak{n}} & =-\frac{k_{\mathrm{rn}}}{\mu_{\mathrm{n}}} K\left(\nabla p_{\mathfrak{n}}-\rho_{\mathfrak{n}} \mathbf{g}\right) .
\end{aligned}
$$$$
(2.44 d)
$$

This model will be used for the rest of this work. Saturation and pressure are coupled by

$$
\begin{aligned}
& S_{w}+S_{n}=1, \quad \text { and } \\
& p_{n}-p_{w}=p_{c},
\end{aligned}
$$

which makes only two out of the four variables $p_{w}, p_{n}, S_{w}$, and $S_{n}$ independent variables.

The model has to be completed by appropriate boundary conditions and initial conditions.
(2.47a)
$S_{w}(x, t)=S_{w d}(x, t) \quad$ on $\Gamma_{w d}^{S}$
Dirichlet BC for saturation
$(2.47 \mathrm{~b})$
$S_{n}(x, t)=S_{n d}(x, t)$
on $\Gamma_{\text {nd }}^{S}$
$(2.47 \mathrm{c})$
$p_{w}(x, t)=p_{w d}(x, t)$
on $\Gamma_{w \mathrm{~d}}^{\mathrm{p}}$
Dirichlet $\mathrm{BC}$ for pressure
$(2.47 \mathrm{~d})$
$p_{n}(x, t)=p_{n d}(x, t)$
on $\Gamma_{\mathrm{nd}}^{\mathrm{p}}$ 


\section{The Model of Fluid Flow in Fractured Porous Media}

\begin{tabular}{|c|c|c|c|}
\hline$(2.47 \mathrm{e})$ & $\rho_{w} \mathbf{u}_{w} \cdot \mathfrak{n}=\phi_{w}$ & on $\Gamma_{w n}$ & Neumann boundary condition \\
\hline$(2.47 f)$ & $\rho_{\mathrm{n}} \mathbf{u}_{\mathrm{n}} \cdot \mathfrak{n}=\phi_{\mathrm{n}}$ & on $\Gamma_{\mathrm{nn}}$ & \\
\hline$(2.47 \mathrm{~g})$ & $S_{w}(x, 0)=S_{w 0}(x)$ & in $\Omega$ & initial conditions \\
\hline$(2.47 \mathrm{~h})$ & $S_{n}(x, 0)=S_{n 0}(x)$ & in $\Omega$ & \\
\hline$(2.47 \mathrm{i})$ & $p_{w}(x, 0)=p_{w 0}(x)$ & in $\Omega$ & \\
\hline$(2.47 \mathrm{j})$ & $p_{n}(x, 0)=p_{n 0}(x)$ & in $\Omega$ & \\
\hline
\end{tabular}

Depending on the choice of independent variables, only a subset of the boundary and initial conditions are necessary. In section 3.4 we explain our choice of variables and comment on the resulting requirements for the boundary and initial conditions.

\subsubsection{InTERFACE CONDITIONS AT Media Discontinuities}

The governing equations for two-phase fluid flow in porous media are only valid if the media properties are subject to slow and smooth variation. However, material parameters are strongly discontinuous between rock matrix and fractures; Wu et al. (2002) report differences between matrix absolute permeability and fracture absolute permeability ranging from a factor 2 up to nine orders of magnitude. At media discontinuities with sharp changes in properties like permeability or porosity it is necessary to introduce interface conditions which model the correct physical behavior. This has been done in van Duijn et al. (I995); we adapt their results for fractured media here.

The significant influence of the capillary pressure on the fluid flow especially at media discontinuities has been shown in laboratory experiments Dawe et al. (1992), Kueper et al. (1989). These results indicate that it is especially important to capture the effects of the capillary forces in the description of the interface conditions since they are responsible for trapping and pooling at media discontinuities.

The partial differential equations 2.44) for two-phase flow are of second order in space and an interface condition at an inner boundary does therefore have to consist of two conditions. The first condition is stated by requiring fluid conservation across medium discontinuities for the wetting phase $w$ and the nonwetting phase $\mathrm{n}$, so for all interfaces $\Gamma$ with associated vector normal $\mathfrak{n}$ we require that

$$
\rho_{w} \mathbf{u}_{w} \cdot \mathfrak{n} \text { and } \rho_{g} \mathbf{u}_{\mathrm{g}} \cdot \mathfrak{n} \text { are continuous across } \Gamma .
$$

Otherwise, mass would be produced or lost at the interface.

For the derivation of the second condition we consider two parts of the domain, a filled fracture $\Omega^{f}$ and the matrix $\Omega^{\mathfrak{m}}$. (The derivation is the same if we consider lenses or layers of different materials.) The absolute permeabilities in their respective domains are

$$
K(x)= \begin{cases}K^{f}(x) & \text { if } x \in \Omega^{f}, \\ K^{m}(x) & \text { if } x \in \Omega^{m} .\end{cases}
$$

Accordingly, the porosity $\Phi$ depends on the domain as well as the capillary pressure function $p_{c}\left(S_{w}\right)$ and the relative permeability functions $k_{r \alpha}$. The capillary pressure functions $p_{c}\left(S_{w}\right)$ 

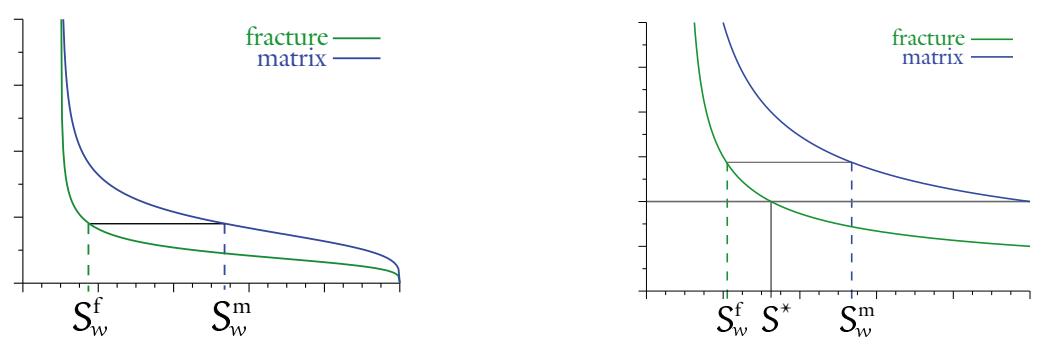

Figure 2.I3.: Capillary pressure curves in a rock matrix and a fracture of higher permeability according to the Van Genuchten model (left) and the Brooks-Corey model (right)

look like the functions in figure 2.I3, depending on whether we choose the Van Genuchten or the Brooks-Corey relation.

For the Van Genuchten relation it is possible to determine for every given nonnegative value of $p_{c}$ the corresponding $S_{w}^{f}$ and $S_{w}^{m}$ by $S_{w}^{f}=\left(p_{c}^{f}\right)^{-1}$ and $S_{w}^{m}=\left(p_{c}^{m}\right)^{-1}$, i. e. we know the wetting phase saturation on either side of the boundary if we know the capillary pressure at the interface. So for the case of a Van Genuchten relation, the second interface condition is continuity of the capillary pressure.

For the Brooks-Corey capillary pressure relation, the entry pressure is positive, and there is a saturation $S_{w}^{\star}$ such that continuity of the capillary pressure can only be achieved if $S_{w} \leq$ $S_{w}^{\star}$. van Duijn et al. (1995) show for a one-dimensional problem, that for $S_{w}>S_{w}^{\star}$ the capillary pressure is discontinuous and that $S_{w}$ is 1 in the matrix $\Omega^{m}$. Physically, if the nonwetting phase is not present (i.e. $S_{w}=1$ and $S_{n}=0$ ), then $p_{n}$ is undefined and $p_{c}$ is discontinuous ( $p_{c}$ is not physically meaningful any more). The proposed interface condition is called extended capillary pressure condition and is

$$
S_{w}^{\mathfrak{m}}= \begin{cases}1 & \text { if } S_{w}^{f}>S_{w}^{\star} \\ \left(p_{c}^{\mathfrak{m}}\right)^{-1}\left(p_{c}^{f}\left(S_{w}^{f}\right)\right) & \text { else. }\end{cases}
$$

\subsection{Models for Fractured Porous Media}

Models for the combined consideration of processes in the fracture network and the matrix can generally be distinguished into discrete models and continuum models (or equivalence mod$e l s$ ). In the continuum model the domain is homogenized based on assumptions about the regularity of fractures. This process can be applied to different scales from the homogenization of microfractures to the homogenization of large fractures on the local scale. In the discrete model the processes are assumed to be dominated by fractures in a way that makes homogenization impossible and the fractures have to be treated explicitly with a "local" fracture continuum. Both approaches were developed to allow for the numerical solution of the resulting equations; in the case of simple equivalence models analytic solutions may also be possible. 


\section{The Model of Fluid Flow in Fractured Porous Media}

\subsection{EQuivalence Models}

Equivalence models are employed if parts of the fractured system or even the whole system are accessible to homogenization. The simplest equivalence model is the single continuum model, in which the fracture network and the rock matrix is homogenized into a medium with an appropriate choice of effective parameters.

The paper of Barenblatt et al. (I960) introduced the double-porosity model (or multi-continumm model). This model separates the fractured rock into two porous systems, the fracture network and the rock matrix. The distinguishing properties of these porous systems are

$\triangleright$ high permeability and low storage capacity in the fractures, and

$\triangleright$ low permeability and high storage capacity in the matrix.

Fluid flow takes place in each system separately, and at the interface between the two systems exchange of fluids can take place through the introduction of an exchange term. The fluid flow in each system is modeled by the classical continuum approach as described in the previous sections. A direct implication is that two separate REvs have to be considered, one for the fractures and one for the matrix.

The model according to Barenblatt et al. (1960) consists of two separate equations for the matrix and the fracture network. The fluid is assumed to be slightly compressible which allows for a simplification of the equation of state for fluids (the situation for gases is more complicated)

$$
\rho=\rho_{0} e^{c\left(p-p_{0}\right)} \quad \text { to } \rho \simeq \rho_{0}+c \rho_{0}\left(p-p_{0}\right),
$$

by series expansion and dropping higher order terms. $\mathrm{c}$ is the compressibility factor, $\rho_{0}$ and $p_{0}$ denote some reference state. We can then formulate the equation of continuity in the matrix and the fractures separately by inserting equation 2.5I (following Sahimi (I995); in Hornung (1997) the equations are formulated with densities only) and arrive at a system

$$
\begin{aligned}
c^{m} \mu \Phi^{m} \frac{\partial p^{m}}{\partial t}-\nabla \cdot\left(K^{m} \nabla p^{m}\right)+\alpha\left(p^{m}-p^{f}\right) & =0, \\
c^{f} \mu \Phi^{f} \frac{\partial p^{f}}{\partial t}-\nabla \cdot\left(K^{f} \nabla p^{f}\right)+\alpha\left(p^{f}-p^{m}\right) & =0 .
\end{aligned}
$$

Upper script $m$ denotes the matrix equation, upper script $f$ denotes the fracture equation. We do not consider source and sink terms here. The third terms on the left hand sides are exchange terms. From their form it is clear that they are assumed to describe a steady state process.

Now two important assumptions are made. The first is that the fracture permeability is much larger than the matrix permeability, $\mathrm{K}^{f} \gg \mathrm{K}^{\mathrm{m}}$, and that from this we can set $\mathrm{K}^{\mathrm{m}}=0$. This reduces the matrix space to a pure storage volume; no fluid flow can take place there. The second assumption, that the storage capacity of the matrix is much higher than the storage capacity of the fractures, translates into the porosity of the matrix space being much larger than the porosity of the fracture space (note that the fracture space is embedded in the 


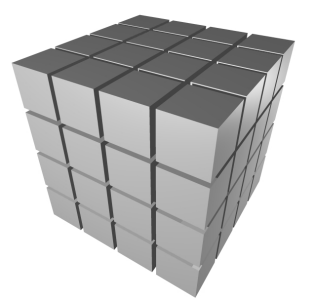

Figure 2.I4.: Disconnected matrix blocks in the double porosity model.

matrix space), i. e. we have $\Phi_{m} \gg \Phi^{f}$ and consequently $\Phi^{f}=0$. With these assumptions, the equations can be specialized further.

The assumption of $\mathrm{K}^{\mathrm{m}}=0$ can also be motivated for the case of totally fissured media. In this model, depicted in figure 2.I4, the matrix blocks are disconnected from each other by the fracture network. Because no direct flow can take place from one matrix cell to another, $\mathrm{K}^{\mathrm{m}}$ is set to 0 .

The obvious limitations of the model led to the development of several extensions. One of the earliest and best known was done by Warren and Root (I963). They considered an idealized setup of a fully connected fracture network with regular parallelepiped blocks (shown in figure 2.I4). Other than Barenblatt et al. (I960) they did not neglect the storage capacity of the fractures. In their model, some of the systems parameters can be estimated from the size and shape of the matrix blocks.

Although the double-porosity model is not necessarily restricted to regular blocks, the majority of double-porosity models has been used for periodic fracture networks. The model has helped the understanding of fluid flow in uniform fracture networks and analytical solutions can be derived for a large number of configurations (Chen, 1989, for an overview), but the complex characteristics of fractures pose a severe limitation to the applicability of these models - the double-porosity model and its refinements contain too many adjustable parameters of which "some have no clear physical meaning and no method of estimating them" (Singhal and Gupta, I999). Some extensions considered the exchange terms and proposed a dependence on the pressure gradient instead of the pressure (Kazemi, I969). But no matter how accurate the exchange term is, it can not hide the fact that a new modeling parameter is introduced into the equations.

Multi-porosity models extend the model by differentiating between two or more matrix species with different permeabilities. In the triple-porosity model, a system of dominant fractures intercepts a system of less pervasive and nested fractures, which is set within a porous matrix. Alternatively, the fractures are located in a configuration of matrices with different porosities and permeabilities (Abdassah and Ershaghi, I986)

Another refinement of the double-porosity model is the dual-permeability model of Hill and Thomas (I985), which considers the rock matrix not only as a storage term but allows for transmissivity in the matrix. By ignoring the interblock transmissivity, the model reduces to the double-porosity model.

In the dual-permeability model of Clemo and Smith (1989) those fractures in a fracture net- 


\section{The Model of Fluid Flow in Fractured Porous Media}

work which are most important to fluid flow are modeled explicitly (primary fractures) while the large majority of remaining, smaller fractures are modeled by network with a lumpedparameter representation. The model relies on stochastic residence time functions which determine the interchange between network blocks, and on probability functions which model the entrance into a network block. The large number of adjustable parameters makes the model capable of reproducing measured data, but the introduction of more modeling parameters and the interpretation step in which the most important fractures are selected is exactly what we want to circumvent.

A mathematical analysis of the double-porosity model was given in Arbogast (1989), $\mathrm{Ar}-$ bogast et al. (1990), Arbogast et al. (I99I). The derivation can be found for single-phase fluids in Allaire (1997) and for two-phase flow in Bourgeat (1997). Computational aspects are considered in Arbogast (1997).

\subsubsection{Discrete Modeling and Numerical Simulation}

An important reason for the development of equivalence models was the desire to make the problems accessible to numerical simulation. An important concept is the usage of lowerdimensional elements in the finite element method for the numerical solution of fluid flow. Lower-dimensional elements, called shell elements, have found widespread acceptance for many problems in mechanical engineering (Ciarlet, I978). This concept has been applied for the numerical simulation of fracture networks which neglect the influence of the matrix altogether and only model fluid flow in the fracture network. Apart from this approach, which is only justified for rocks with very small matrix conductivity, there have been finite element models which employ elements of different dimensionality. The term discrete model can be found in the literature for both approaches; to distinguish both we refer to the former by discrete fracture network model. The combination of discrete fracture network models with a continuum model is also known as hybrid models. We employ the term discrete model for our approach. Wilson and Witherspoon (1974) was one of the earliest papers on numerical simulation of water flow in fractured porous media. It contains two finite element models, one with two-dimensional elements for rock matrix and fractures and one model with onedimensional elements for the fracture network which does not take the rock matrix into account. Gureghian (1975) formulated a finite element model in three space dimensions with tetrahedral elements in the rock matrix and triangular elements in the fractures; a similar approach is presented in Baca et al. (1984) and in Eaton and Bixler (1987). The approach of element types of different dimension is also pursued in Woodbury and Zhang (200I), Sudicky and McLaren (1998).

The investigation of unsaturated flow received less attention in the past. J. S. Y. Wang and T. N. Narasimhan (1985) modeled unsaturated flow with a discrete fracture approach. Berkowitz et al. (I988) considered solute transport in a fractured porous medium with discrete fractures modeled by one-dimensional equations and the matrix modeled by twodimensional equations, coupled by exchange terms. In Helmig (I993) mixed-dimensional elements were employed for two-phase flow; see also ROCKFLOW $(1986-2003)$. 


\subsection{Approach taken in this Work}

\subsection{APPROACH TAKEN IN THIS WORK}

The present chapter has explained some of the many difficulties that arise in the modeling of fluid flow and transport in fractured porous media. It should be obvious that many assumptions and simplifications have to be imposed on every model that tries to give a description of the processes on a large scale. In this section we present our assumptions that we impose on the model. These assumptions will be essential for the applicability of the numerical simulation.

I. We assume that fracture width is orders of magnitude smaller than the fracture length. Fractures are of essentially planar geometry, with an associated aperture in each point.

2. We assume that the multiphase fluid flow equations (2.44) are valid in the rock matrix and the fractures, i. e. the multiphase extension of Darcy's law is valid. This implies that we assume a laminar flow regime in both domains and that an REV can be found for fracture and matrix. Multi-component and non-isothermal behavior of the fluids is not considered.

3. The absolute permeability of the fractures is larger than the absolute permeability of the rock matrix. Fractures may be open or filled, but we do not consider blocking fractures.

4. Relative permeability functions and capillary pressure functions exist for fractures and matrix. The capillary pressure function is assumed to be strictly monotone decreasing, and we assume that the capillary pressure functions for rock and matrix do not intersect. 
2. The Model of Fluid Flow in Fractured Porous Media 


\section{Finite Volume Discretization and Multigrid SOLUTION}

A mathematical description of the equations which model two-phase flow in a fractured porous medium was developed in the previous chapter, and the purpose of this chapter is to present an efficient scheme for the numerical solution of the two-phase flow equations. To device an efficient scheme, we need to determine the nature of the equations, i. e. we need to determine whether the equations are of elliptic, parabolic or hyperbolic type. This classification affects the choice of the discretization method from the broad range of algorithms for spatial and temporal discretization.

The classification is carried out in section 3.2, section 3.I gives a short overview over existing schemes for the numerical solution of partial differential equations. After selecting an appropriate formulation in section 3.4, we present a fully implicit, fully coupled vertexcentered finite volume method for the two-phase flow equations. This is the main part of this chapter. The remainder of the chapter is concerned with the efficient solution of the systems of equations arising from the discretization by an inexact Newton method and a linear multigrid method.

\section{I. Numerical SOLUTION SCHEMES FOR THE TWO-PHASE EQUATIONS}

There are several difficulties which have to be dealt with in approaches to the numerical simulation for subsurface problems. We draw from the accurate description of Ewing (1983) and Bastian (1999) and give a short overview over some of the available numerical schemes. Based on the difficulties we must expect to encounter and the properties of the available schemes, we motivate our choice.

The analysis of the two-phase flow equations in section 3.2 reveals the nonlinear convection-diffusion nature of the saturation equation. This is the more demanding part in the simulation process, the treatment of the elliptic equation (the pressure equation) is comparatively easy. Application of centered differences or standard Galerkin finite element methods to transport-dominated parabolic equations yields second order accurate schemes, but the solution can contain oscillations for non-smooth solutions. Stabilization by upwinding makes the solution monotone, but it reduces the approximation order, introduces grid dependencies into the solution and smears out sharp fronts appearing in the solution.

Capillary effects are normally neglected in reservoir engineering applications, but they play an important role on a smaller scale. For non-vanishing capillary pressure effects, the saturation equation is of degenerate parabolic type (instead of hyperbolic) and the numerical scheme must appropriately handle the free boundary in the solution. Capillary pressure 


\section{Finite Volume Discretization and Multigrid Solution}

effects become especially apparent in the treatment of media discontinuities (to which we count fractures). Many numerical methods fail to reproduce the behavior of pooling, entrapment and discontinuous saturations at media discontinuities (Helmig, I997).

In the formulation in section 3.2 , the saturation equation is coupled to the pressure equation by the velocity derived from the pressure. An accurate calculation of the fluid velocities is therefore crucial for the numerical scheme.

If the frontal mobility ratio is greater than one, the macroscopic equations are unstable and fingering develops. This is due to numerical errors, and does not approximate the fingering process which occurs in the physical problem due to microscopic instabilities.

The time differencing scheme influences the solution behavior and a balanced choice between efficiency and accuracy is difficult and very problem-dependent. Explicit and implicit methods and combinations thereof are available for two-phase flow equations. With implicit schemes, fast solvers for the arising systems of nonlinear equations have to be devised. Since the pressure equation is elliptic, a fully explicit treatment of the two-phase flow problem is not possible.

Numerical simulation of multiphase flow problems has a long and remarkable history (Douglas Jr. et al., 1959, for the first numerical simulator). A widespread approach is the splitting of the treatment of the saturation equation and the pressure equation. After solving the pressure equations (with coefficients depending on saturation), one or several time steps are computed for the saturation equation with a fixed velocity field. The name IMPES (implicit pressure, explicit saturation) is employed for schemes which use explicit time differencing schemes for the saturation equation.

In the convection-dominated case, standard finite difference, finite element or finite volume methods have difficulties, arising either from the temporal discretization error of the backward Euler method, or from the time step size restrictions due to stability problems of the higher order Crank-Nicholson or $\mathrm{BDF}(2)$ schemes. An attractive alternative is the strongly stable fractional-step- $\theta$ scheme, which captures sharp fronts better than the backward Euler scheme.

High-order explicit finite volume schemes (Le Veque, 2002) have a Courant number limitation, but their evaluation is cheap. They have been applied to two-phase flow with and without capillary pressure by Durlofsky (I993) and Helmig and Huber (1996), respectively. The Essentially Non-oscillatory (ENO) and Weighted Essentially Non-oscillatory Schemes (WENO) (Shu, 1999, 1998) are based on the idea to choose an adaptive stencil which guarantees high-order approximation in regions where the solution is smooth. Then the oscillations near shocks decay with the order $\mathcal{O}\left(\Delta x^{k}\right)$ for kth order approximation. However, they have not seen application to complex realistic problems yet.

An appealing approach is offered by the characteristic methods, which consider the characteristics of the hyperbolic part. The modified method of characteristics (MMOC) of Douglas Jr. and Russel (1982) interprets the temporal derivative and the advective part together as a directional derivative in the characteristic direction and then discretizes by a backward difference quotient. The method is capable of very large time steps, but does not conserve mass and has difficulties handling Dirichlet boundary conditions. Non-mass conserving schemes can lead to false predictions of the front position (Hou and LeFloch, 1994). For nonlinear problems, the large time steps can not be taken. Another approach to characteristic meth- 
ods is offered by the Eulerian-Lagrangian localized adjoint method (ELLAM) of Celia et al. (I990). ELLAM-type methods are mass-conservative and can treat all kinds of boundary conditions. The method uses a weighted residual formulation and chooses weight functions which have local support and solve the homogeneous adjoint equations in the interior of each space-time element. Treatment of multiphase flow problems with ELLAM is explained in Binning and Celia (1994) and Ewing (I99I), but no numerical results are given. It turns out that the implementation of the characteristic tracking algorithm necessary for ELLAM schemes is already very difficult for general domains in two space dimensions.

The upcoming class of discretization methods by the name discontinuous Galerkin methods is reviewed in chapter 4. See the introductions by Cockburn (1999, I998) and the articles in Cockburn et al. (2000b) for an overview.

If the numerical simulator is supposed to handle a wide range of applications, fully coupled and fully implicit schemes which employ implicit time differencing schemes on the full system of equations are known to be very robust. The resulting equations have to be solved with a Newton method or quasi-Newton method. The robustness of the method makes it very attractive for our fields of application.

The number of discretization methods that have been combined with the fully coupled, fully implicit approach is far too large to allow a complete presentation. Apart from standard finite element methods and hp-finite element methods (Schwab, I999), there are controlvolume finite elements (Forsyth, I99I) and streamline diffusion methods (Brooks and Hughes, I982). Highly popular, due to their mass conserving and monotone behavior, are finite volume methods. An overview can be found in Michev (I996). Different fully implicit methods were compared in Helmig (1997).

The choice of our method was directed by the following considerations:

$\triangleright$ The simulator should be applied to problems in fractured porous media from the laboratory scale to the field scale. We need to be able to capture the effects of capillary pressure; this is done by including the extended capillary pressure condition of van Duijn et al. (I995). The simulator should be able to handle a compressible gas phase. An extension of the functionality to three-phase flow, multicomponent flow, isothermal problems,... should be possible.

$\triangleright$ The domains we encounter are usually very complex and unstructured meshes are absolutely necessary. This precludes schemes which only work on structured grids.

$\triangleright$ The numerical scheme should be stable, consistent, monotone and mass conservative. Monotonicity is important because the saturation values must lie between 0 and 1. Methods which are not locally mass conservative can fail to predict the correct location of shocks or sharp fronts (Hou and LeFloch, 1994, Le Veque, 2002).

$\triangleright$ For the time discretization we employ the backward Euler method and the fractionalstep- $\theta$ method. Both methods are strongly A-stable.

$\triangleright$ The key ingredient for an efficient implicit scheme is the fast solution of the nonlinear systems of equations. We use an inexact Newton scheme. The scheme is inexact in that 


\section{Finite Volume Discretization and Multigrid Solution}

it only solves the arising linear systems of equations up to a given tolerance. Global convergence of the Newton method is achieved by a line-search algorithm. The linear systems of equations are solved with a multigrid method.

$\triangleright$ Efficiency and the memory requirements of realistic applications demand to run the simulator on parallel computers with message passing architecture. Adaptivity can greatly reduce run-time and memory requirements.

$\triangleright$ The software complexity of a project with the mentioned features is by orders of magnitude too large to be handled by a single person. We based our development on the software toolkit UG (Bastian et al., 1997).

\subsection{Character of the EQUations}

A successful numerical scheme for the solution of the two-phase flow equations can not be designed without careful consideration of the character of the equations. A first look at equations 2.44) seems to show two parabolic equations with diffusion-like properties. But the character is more complex, as a transformation by Chavent (I976, I98I) revealed. The transformation assumes that the densities of both phases and the porosity are constantsakin to the assumption that fluids and rock are incompressible - and that there are no gravity terms. It then defines the total mobility

$$
\lambda\left(S_{w}, S_{n}\right)=\frac{k_{r n}}{\rho_{n}}+\frac{k_{r w}}{\rho_{w}}=\frac{k_{r n}\left(S_{n}\right)}{\rho_{n}}+\frac{k_{r w}\left(S_{w}\right)}{\rho_{w}},
$$

the phase mobility for the phases $\alpha=n, w$

$$
\lambda_{\alpha}\left(S_{w}, S_{n}\right)=\frac{k_{r \alpha}}{\lambda \rho_{\alpha}}=\frac{k_{r \alpha}\left(S_{\alpha}\right)}{\lambda\left(S_{w}, S_{n}\right) \rho_{\alpha}},
$$

the average pressure

$$
p=\frac{1}{2}\left(p_{w}+p_{n}\right)+\frac{1}{2} \int_{0}^{p_{c}}\left(\lambda_{n}(\xi)-\lambda_{w}(\xi)\right) d \xi,
$$

and the total fluid velocity

$$
\tilde{u}=-K(x) \lambda\left(S_{w}, S_{n}\right) \nabla p .
$$

If we add and subtract the two-phase flow equations 2.44) and collect terms as in Douglas jir. (1983), we arrive at the system

$$
\begin{aligned}
& \nabla \cdot \tilde{u} \equiv-K(x) \lambda\left(S_{w}, S_{n}\right) \nabla p=\frac{q_{n}}{\rho_{n}}+\frac{q_{w}}{\rho_{w}}, \\
& \Phi \frac{\partial S_{w}}{\partial t}-\nabla \cdot\left(K \lambda \lambda_{w} \lambda_{n} \frac{d p_{c}}{d S_{w}} \nabla S_{w}\right)+\lambda_{w} \tilde{u} \cdot \nabla S_{w}=f\left(q_{w}, \rho_{w}, \lambda_{w}, q_{n}, \rho_{n}, \lambda_{n}\right) .
\end{aligned}
$$


$f$ is a linear function of the flow properties.

Equation (3.5a) is of elliptic type. Without the assumption that the densities of both phases and the porosity are constants, the equation takes the form of a parabolic equation, where the total compressibility of the system enters in the time derivative term; if the total compressibility is very small (as is often the case), the character of the equation is elliptic or almost elliptic.

Equation 3.5 b looks like a nonlinear convection-diffusion equation. If $S_{w}=0$ or $S_{n}=0$ the corresponding mobilities $\lambda_{w}$ and $\lambda_{n}$ vanish and the coefficients of the diffusion term degenerate in this case. The diffusion term also degenerates if capillary effects are small. In both cases the equation is of degenerate parabolic type. Degenerate parabolic equations have some of the same properties as hyperbolic equations, such as travelling wave fronts. Fronts moving through the medium will be slightly diffused by capillary pressure effects, and fingering can occur as a result of viscosity differences.

If the capillary pressure gradient $d p_{c} / \mathrm{dS}_{w}$ is zero, the equation $3.5 \mathrm{~b}$ ) is nonlinear hyperbolic. Since the treatment of the capillary pressure is a key component of this work, we will only briefly comment on this case.

The determination of the character of the equations does not answer the question if a solution to the equations exists. Theoretical analysis of the two-phase flow equations is in most cases done for the global pressure formulation. This formulation is discussed in detail in Chavent and Jaffré (I978), where also solutions to some variational formulations of the global pressure formulation are presented for the degenerate and non-degenerate incompressible case. Also in Chavent and Jaffré (1978), uniqueness was shown in the case of complete decoupling of pressure and saturation. Solutions to the degenerate parabolic equation have very low regularity (Yotov, I997). In Schroll and Tveito (1997) the existence of classical solutions for the incompressible elliptic-hyperbolic system (with $p_{c} \equiv 0$ ) was shown. Existence of a weak solution to 2.44) was shown in Kroener and Luckhaus (1984) under the assumption that $S_{e}$ is bounded away from 0 for Dirichlet and mixed boundary conditions.

\subsection{Numerical Methods For hyperbolic AND DEGENERATE PARABOLIC EQUATIONS}

\subsection{NONLINEAR HyPERbolic EQUATIONS}

The global pressure formulation can be reduced in the case of two incompressible fluids in one space dimension without gravity and source terms. By imposing the boundary and initial conditions

$$
S_{w}(0, t)=S \quad S_{w}(x, 0)=S_{w 0}(x)
$$

the problem can be written in the form known as the Buckley-Leverett equation:

$$
\frac{\partial S_{w}}{\partial t}+\frac{\partial}{\partial x} f\left(S_{w}\right)=0
$$




\section{Finite Volume Discretization and Multigrid Solution}

with the nonlinear fractional flow function $\mathrm{f}$,

$$
f\left(S_{w}\right)=\frac{u}{\Phi} \frac{k_{r w}\left(S_{w}\right)}{k_{r w}\left(S_{w}\right)+\frac{\mu_{w}}{\mu_{g}} k_{r g}\left(1-S_{w}\right)}
$$

$\mathrm{U}$ is the constant total velocity. The Buckley-Leverett problem as a simple example of a nonlinear hyperbolic equation gets extensive treatment in several excellent sources: Le Veque (1992) and the recent, largely upgraded monograph Le Veque (2002) are devoted to hyperbolic equations. See also Bear (1972), Renardy and Rogers (I993), Bastian (1999).

Some of the important insights gained from the hyperbolic case, which are covered in the above references, are as follows.

Front CAPTURING. Solutions to hyperbolic equations can contain discontinuities, which in the case of nonlinear hyperbolic equations can arise even from smooth initial data. Much of the work on the numerical solution of hyperbolic equations is concerned with the treatment of discontinuouities or sharp fronts. A successful numerical schemes should be able to represent the sharp front without the addition of artificial diffusion and it should avoid to produce oscillations near the shock. Oscillations occur for higher order methods, and many high resolution methods have been developed based on flux limiters (Le Veque, 2002). In the case of nonlinear hyperbolic equations the smearing of sharp fronts in the numerical solution can be weakened due to the self-sharpening effect arising from the nonlinearities. An important concept is the TVD property (total variation diminishing), which-if satisfiedprevents oscillations near a shock.

Stability. A necessary (though not sufficient) condition for the stability of a numerical scheme for the solution of hyperbolic equations was presented in the landmark paper by Courant et al. (1928) and is named Courant-Friedrichs-Lewy condition or CFL condition:

$$
\frac{\left|v_{\max }\right| \Delta t}{\Delta x}<1
$$

It describes the maximal possible time step $\Delta t .\left|v_{\max }\right|$ is the maximum velocity.

ENTROPY SOLUTIONS AND JUMP CONDITIONS In some situations the weak solution to a hyperbolic equation is not unique and an additional condition is required to find the physically relevant solution. This is the vanishing viscosity solution which is the solution obtained by considering an additional viscosity term and choosing the limiting solution for vanishing viscosity. Convergence to this solution can be obtained by imposing an entropy condition; the resulting solution is called the entropy solution. An additional requirement in the case of nonlinear hyperbolic equations is that the shock jump must obey the Rankine-Hugoniot condition.

FINGERING. The frontal mobility ratio is defined by

$$
M=\frac{k_{\mathrm{r} \alpha}(S \triangleright)}{k_{\mathrm{r} \beta}(\triangleright S)} \cdot \frac{\mu_{\beta}}{\mu_{\alpha}},
$$



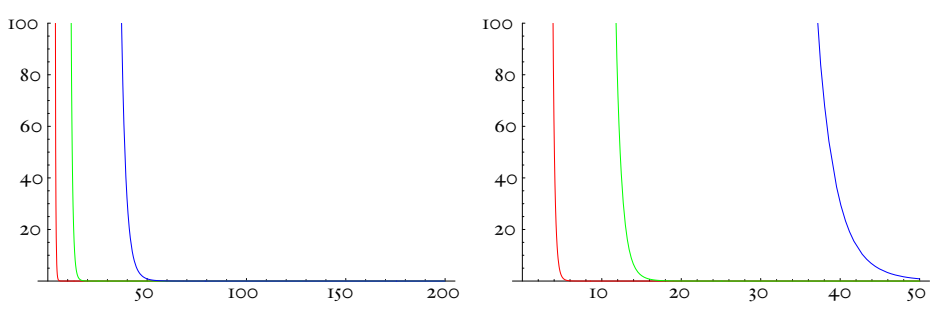

Figure 3.I.: Solutions to the porous medium equation (3.II) by separation of variables (left) and Barenblatt's solution (right) for $t=1,10,100$.

where $\alpha$ denotes the displacing fluid and $\beta$ the displaced fluid. $S \triangleright$ is the saturation of the displacing fluid behind the front, $\triangleright S$ is the saturation of the displaced fluid ahead of the front. If $M>1$, the position of the shock front is unstable in the multidimensional case (Bear, 1972, Kueper and Frind, I988, Glimm et al., 1981, 1983). This leads to the effect of fingering in the solution.

\subsubsection{Degenerate parabolic equations}

A simple example for a degenerate parabolic equation is the porous medium equation (Evans, 1998). It has the form of a nonlinear diffusion equation,

$$
\partial_{\mathrm{t}} \mathfrak{u}-\Delta\left(\mathrm{u}^{\gamma}\right)=0 \quad \text { in } \mathbb{R}^{\mathrm{d}} \times(0, \infty)
$$

with $u \geq 0$ and $\gamma>1$. An analytic solution to this equation can be found by separation of variables, which yields the solution

$$
\begin{gathered}
\alpha=\frac{2}{\gamma-1} \quad \mu=\alpha \gamma(\alpha \gamma+d-2) \\
u(x, t)=((1-\gamma) \mu t+\lambda)^{\frac{1}{1-\gamma}}|x|^{\alpha},
\end{gathered}
$$

with a constant $\lambda>0$. Another solution was found by Barenblatt,

$$
\begin{gathered}
\alpha=\frac{d}{d(\gamma-1)+2} \quad \beta=\frac{1}{d(\gamma-1)+2} \\
u(x, t)=\frac{1}{t^{\alpha}}\left(b-\frac{\gamma-1}{2 \gamma} \beta \frac{|x|}{t^{2 \beta}}\right)^{\frac{1}{1-\gamma}},
\end{gathered}
$$

with a constant $b$. In figure $3 . \mathrm{I}$ the solution curves for $\gamma=7 / 8, d=2, b=1, \lambda=2$, $t=1,10,100$ are plotted. A feature of the solution $u$ of $3 . \mathrm{II}$ is that it has compact support for $t>0$ and that the partial differential equation becomes degenerate for $u=0$. The set $\{u>0\}$ moves with finite propagation speed, which is why it is sometimes considered a better model of diffusive spreading than the linear heat equation (according to which an infinite information propagation speed is possible). 


\section{Finite Volume Discretization and Multigrid Solution}

An example for a parabolic equation that can be derived from the two-phase flow equations (2.44) is the McWhorter problem by McWhorter and Sunada (1990); it is also treated in Bastian (I999), Helmig (I997). For the unknown $S_{w}$ the McWhorter problem reads

$$
\Phi \frac{\partial S_{w}}{\partial t}+\frac{\partial}{\partial x}\left(\lambda_{n} f_{w} \frac{d p_{c}}{d S_{w}} K \frac{\partial S_{w}}{\partial x}\right)
$$

The numerical treatment of parabolic equations is presented in Thomée (1997).

\subsection{Phase pressure-Saturation formulation}

The transformation of the multi-phase flow equations 2.44 in section 3.2 is not applicable to our field of investigation, because the gas phase is compressible. Instead we choose a fully coupled approach based on the Phase pressure-Saturation formulation. As already mentioned, only two out of the four variables $p_{w}, p_{g}, S_{w}$, and $S_{g}$ in the two equations 2.44 can be chosen as independent variables. We choose the substitutions

$$
S_{w}=1-S_{g}, \quad p_{g}=p_{w}+p_{c}\left(1-S_{g}\right)
$$

to obtain the $\left(\mathrm{p}_{w}, \mathrm{~S}_{\mathrm{g}}\right)$-formulation. Other choices are possible, see Helmig (1997). We would like to note that the difference between the $\left(\mathrm{p}_{w}, S_{g}\right)$ formulation and the $\left(\mathrm{p}_{w}, S_{w}\right)$ formulation reduces to inserting $1-S_{w}$ for $S_{g}$. Because of this, we write $S_{w}$ and $S_{g}$ in the following to facilitate reading. Of course the same argumentation is not applicable for $p_{w}$ and $p_{g}$, because the choice of $p_{w}$ as an independent variable has larger implications. Formulations based on $p_{w}$ assume that the water phase is present everywhere in the domain. Because we consider problems in initially fully water-saturated domains with a residual water saturation (i. e. the water phase is never completely replaced by gas) this is the appropriate choice.

The equations now read

$$
\begin{array}{ll}
\frac{\partial\left(\Phi \rho_{w}\left(S_{w}\right)\right)}{\partial t}-\nabla \cdot\left(\rho_{w} \frac{k_{r w}}{\mu_{w}} K\left(\nabla p_{w}-\rho_{w} \mathbf{g}\right)\right) & -\rho_{w} q_{w}=0 \\
\frac{\partial\left(\Phi \rho_{g}\left(S_{g}\right)\right)}{\partial t}-\nabla \cdot\left(\rho_{g} \frac{k_{r g}}{\mu_{g}} K\left(\nabla p_{w}+\nabla p_{c}\left(S_{w}\right)-\rho_{g} g\right)\right)-\rho_{g} q_{g}=0
\end{array}
$$

where we used $S_{w}$ for $1-S_{g}$. We consider these equations in $(0, T) \times \Omega . \Omega \subset \mathbb{R}^{d},(d=2,3)$ is a domain with polygonal or polyhedral boundary for $\mathrm{d}=2$ and $\mathrm{d}=3$ respectively. The equations are complemented with initial conditions and boundary conditions of Neumann or Dirichlet type

$$
\begin{aligned}
p_{w}(x, 0) & =p_{w 0}(x) \\
p_{w}(x, t) & =p_{w d}(x, t) \text { on } \Gamma_{w d} \\
\rho_{w} \boldsymbol{v}_{w} \cdot \mathfrak{n} & =\phi_{w}(x, t) \text { on } \Gamma_{w n}
\end{aligned}
$$

$$
\begin{aligned}
& S_{g}(x, 0)=S_{g 0}(x) \quad \forall x \in \Omega, \\
& S_{g}(x, t)=S_{g d}(x, t) \text { on } \Gamma_{g d},
\end{aligned}
$$$$
\rho_{\mathrm{g}} \boldsymbol{v}_{\mathrm{n}} \cdot \mathrm{n}=\phi_{\mathrm{g}}(x, \mathrm{t}) \text { on } \Gamma_{\mathrm{gn}} \text {. }
$$

If both phases are incompressible no initial condition for $p_{w}$ is required. $\Gamma_{w d}^{p}$ should have positive measure to determine $p_{w}$ uniquely. 


\subsection{The Finite Volume Method}

In the following we assume the dependencies

$$
\begin{aligned}
\mathbf{g} & =\text { constant } & & \mathrm{q}_{\alpha}= & \mathrm{q}_{\alpha}(\boldsymbol{x}, \mathrm{t}) \\
\mathrm{p}_{\mathrm{c}} & =\mathrm{p}_{\mathrm{c}}\left(\boldsymbol{x}, \mathrm{S}_{w}\right) & & \mathrm{k}_{\mathrm{r} \alpha}= & \mathrm{k}_{\mathrm{r} \alpha}\left(\boldsymbol{x}, \mathrm{S}_{\alpha}\right) \\
\rho_{\alpha} & =\rho_{\alpha}\left(\mathrm{p}_{\alpha}\right) & & \mu_{\alpha}= & \mu_{\alpha}\left(p_{\alpha}\right) \\
\Phi & =\Phi(\boldsymbol{x}) & & &
\end{aligned}
$$

The influence of fractures on the fluid flow is included through the dependency of the quantities in equation 3.20 on the position, i. e. the values are different depending on whether they are evaluated in a fracture or in the rock matrix.

A similar derivation as the one leading to 3.18 ) produces the $\left(\mathrm{p}_{\mathrm{g}}, \mathrm{S}_{w}\right)$ formulation. This is the appropriate formulation if $S_{g}$ is bounded away from 0 . The choice of $\left(p_{w}, p_{g}\right)$ as the primary unknowns is called the pressure-pressure formulation or $\left(\mathrm{p}_{w}, \mathrm{p}_{\mathrm{g}}\right)$ formulation. In the $\left(p_{w}, p_{g}\right)$ formulation the saturation is computed from the capillary pressure function by inversion, $S_{w}=p_{c}^{-1}\left(p_{\mathfrak{n}}-p_{w}\right)$. Obviously, the pressure-pressure formulation requires that $S_{w}$ and $S_{g}$ are bounded away from 0 .

\subsection{The Finite Volume Method}

In this section we describe the vertex-centered finite volume method which was devised after the considerations in section 3.I. Finite volume methods are presented in Michev (I996), Bey (I998). Their close relation to the finite element method was revealed in Hackbusch (I989). A recent application to two-phase flow can be found in Michel (2003). The finite volume method is sometimes called finite volume element method. In the engineering literature it is often called Control Volume FEM, Bank and Rose (1987), Hackbusch (1989) use the term box method.

\subsection{Geometry of the problem and the Primary and Secondary Mesh}

In the previous section the domain was only specified with respect to the shape of its boundary and without the description of fractures. Some assumptions on the fracture network which are essential for the discretization method are described. In the following superscript $m$ notation denotes entities in the volumetric rock matrix and superscript $f$ denotes entities in the fracture network.

Let $\Omega \subset \mathbb{R}^{\mathrm{d}}$ be a polygonal or polyhedral domain for $\mathrm{d}=2$ or $\mathrm{d}=3$, respectively. The domain contains a nonempty set of fractures $\left\{f_{1}, \ldots, f_{F}\right\}$. Each fracture $f_{i}$ is a $(d-1)$ dimensional object $-i$. e. we identify each fracture with its middle surface - and each fracture $f_{i}$ has a width $w_{i}$ associated with it, which may be variable in the fracture. For simplicity we assume the fractures to have a planar geometry: In a two-dimensional domain the fractures are line segments and in a three-dimensional domain we assume polygonal shape of the fractures (although circular or elliptic shapes can also be treated by the method, as well as non-planar shapes). The union of the fractures constitutes the fracture network

$$
\Omega^{f}=\bigcup_{i=1}^{F} f_{i} \subset \Omega .
$$




\section{Finite Volume Discretization and Multigrid Solution}

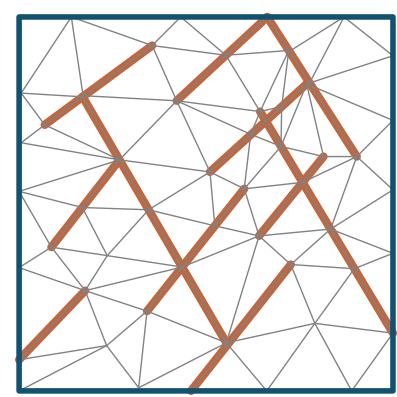

Figure 3.2.: Example domain with fractures and mesh resolving the fracture network geometry.
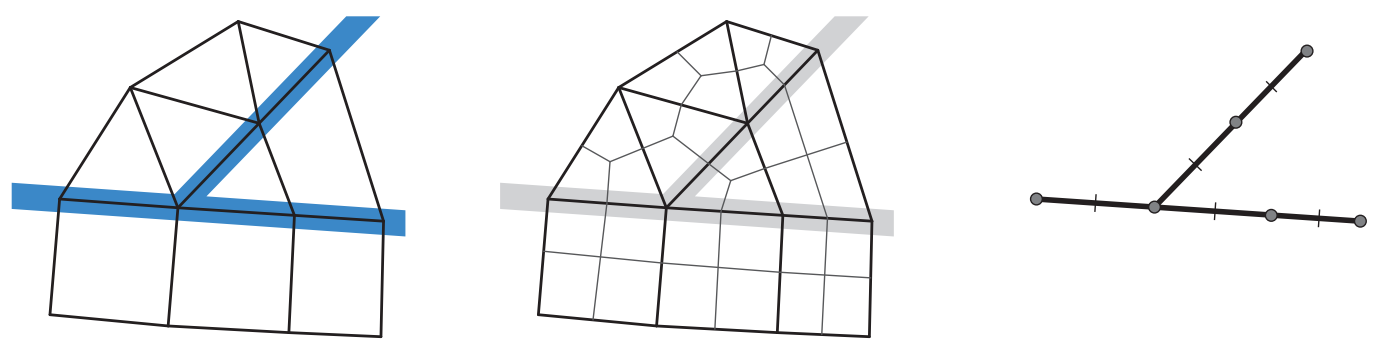

Figure 3.3.: Mesh, dual grid and fracture elements/volumes.

The domain of the rock matrix $\Omega^{\mathrm{m}}$ is the whole domain,

$$
\Omega^{\mathrm{m}}=\Omega .
$$

This means that the domains of the fracture network and the rock matrix overlap.

The discretization method requires a mesh for $\Omega^{\mathrm{m}}$ and $\Omega^{\mathrm{f}}$. For the volumetric mesh we consider a subdivision $\mathrm{E}_{h}^{\mathrm{m}}$ of $\Omega^{\mathrm{m}}$ into $\mathrm{K}$ elements $e, \mathrm{E}_{\mathrm{h}}^{\mathrm{m}}=\left\{e_{1}, \ldots, e_{\mathrm{K}}\right\}$. By $\Omega_{e}$ we denote the subdomain covered by element $e$ and we require $\bigcup_{e} \overline{\Omega_{e}}=\bar{\Omega}$ and $\Omega_{e} \cap \Omega_{f}=\emptyset$ for $e \neq f$. $h$ denotes the diameter of the largest element. The subdivision has to resolve the geometry of the fractures $f_{i}$, comparable to domains with inner boundaries. Figure 3.2 shows an example for a two-dimensional mesh. The volumetric elements $\Omega_{e}$ of $\mathrm{E}_{\mathrm{h}}^{\mathrm{m}}$ are triangles or quadrilaterals in two dimensions and tetrahedrons, pyramids, prisms, or hexahedrons in three dimensions. Hybrid grids, i. e. grids of mixed element type are admissible, for any grid we require that $E_{h}^{m}$ is a triangulation: No vertex of an element lies in the interior of a side of another element.

The volumetric elements are complemented with lower dimensional elements on the fractures which are line elements for two-dimensional problems and triangles or quadrilaterals for three-dimensional problems. The fracture elements constitute a mesh $E_{h}^{f}=\left\{f_{1}, \ldots, f_{K^{f}}\right\}$ which is conforming with the volumetric mesh, i. e. each $\Omega_{e}^{f}$ is an element side or face for the two-dimensional and three-dimensional case, respectively.

The vertex centered finite volume method requires the construction of a secondary mesh 


\subsection{The Finite Volume Method}

$\mathrm{B}_{h}^{m}$. For the volumetric mesh it is constructed by connecting element barycenters with edge midpoints as shown in figure 3.3 in two dimensions. In three dimensions, first the element barycenters are connected to element face barycenters and then these are connected with edge midpoints. Vertices of the grid are denoted by $v_{i}$ and their corresponding coordinate vector by $x_{i}$. By construction each control volume contains exactly one vertex, and the control volume containing vertex $v_{i}$ is denoted by $b_{i}^{m}$. The generation of the dual grid for the fractures happens in the same way for two-dimensional fractures. One-dimensional elements are simply divided into equal parts. This construction results in a conforming dual mesh for volumetric and fracture elements. The fracture dual mesh is denoted $B_{h}^{f}$.

The construction of the dual grid can happen in alternative ways and the requirements on the dual grid are quite general (Bey, I998, Michev, I996).

The internal skeleton of the volumetric dual grid consists of the sides of the control volumes. The edge between control volume $b$ and $b^{\prime}$ inside of element $e$ is denoted by

$$
\gamma_{e, b, b^{\prime}}=\bar{\Omega}_{e} \cap \bar{\Omega}_{b} \cap \bar{\Omega}_{b^{\prime}} \quad \text { for } e \in E_{h}^{m}, b, b^{\prime} \in B_{h}^{m}
$$

and the union of all internal edges of the volumetric dual grid is denoted by

$$
\Gamma_{\text {int }}^{m}=\left\{\gamma_{e, b, b^{\prime}} \mid e \in E_{h}^{m}, b, b^{\prime} \in B_{h}^{m}\right\} .
$$

For the fracture dual grid the intersection of control volume $b$ and $b^{\prime}$ on the edge between elements $e$ and $f$ is

$$
\gamma_{e, f, b, b^{\prime}}=\bar{\Omega}_{e} \cap \bar{\Omega}_{f} \cap \bar{\Omega}_{b} \cap \bar{\Omega}_{b^{\prime}} \quad \text { for } e, f \in E_{h}^{f}, b, b^{\prime} \in B_{h}^{f}
$$

and the union of all internal control volume intersections (which are points for one-dimensional fractures and edges for two-dimensional fractures) of the fracture dual grid is denoted by

$$
\Gamma_{\text {int }}^{f}=\left\{\gamma_{e, f, b, b^{\prime}} \mid e, f \in E_{h}^{f}, b, b^{\prime} \in B_{h}^{f}\right\} .
$$

In the two-dimensional case, $\gamma_{e, f, b, b^{\prime}}$ is already determined uniquely by $e$ and $f\left(\right.$ or $b$ and $b^{\prime}$ ), but in the three-dimensional case all four are needed.

The external skeleton is the union of the element sides on the domain boundary, defined for the volumetric dual mesh and the fracture dual mesh,

$$
\begin{aligned}
& \Gamma_{\mathrm{ext}}^{\mathrm{m}}=\left\{\gamma_{e, \mathrm{~b}} \mid \gamma_{e, \mathrm{~b}}=\partial \Omega_{e} \cap \partial \Omega_{\mathrm{b}} \cap \partial \Omega \text { for } e \in \mathrm{E}_{\mathrm{h}}^{\mathrm{m}}, \quad \mathrm{b} \in \mathrm{B}_{\mathrm{h}}^{\mathrm{m}}\right\}, \\
& \Gamma_{\mathrm{ext}}^{\mathrm{f}}=\left\{\gamma_{e, f, \mathrm{~b}} \mid \gamma_{e, f, b}=\partial \Omega_{e} \cap \partial \Omega_{\mathrm{f}} \cap \partial \Omega_{\mathrm{b}} \cap \partial \Omega \text { for } e \in E_{h}^{f}, \quad b \in B_{h}^{f}\right\} .
\end{aligned}
$$

With each element of the skeleton we associate a fixed unit normal $\boldsymbol{n}$. For $\gamma \in \Gamma_{\mathrm{ext}}^{\mathrm{m}}$ and $\gamma \in \Gamma_{\mathrm{ext}}^{\mathrm{f}}$ we choose the outward unit normal. For interior sides the direction of $\boldsymbol{n}$ is chosen arbitrarily, but fixed.

For any function $f$ defined on $\Omega$, which may be discontinuous on $\Gamma_{\text {int }}^{m}$ and $\Gamma_{\text {int }}^{f}$, we define the jump of $f$ at $x \in \gamma \in \Gamma_{\text {int }}$ to be

$$
[v](\boldsymbol{x})=\lim _{\varepsilon \rightarrow 0+} v(\boldsymbol{x}+\varepsilon \mathfrak{n})-\lim _{\varepsilon \rightarrow 0+} v(\boldsymbol{x}-\varepsilon \mathfrak{n}) .
$$



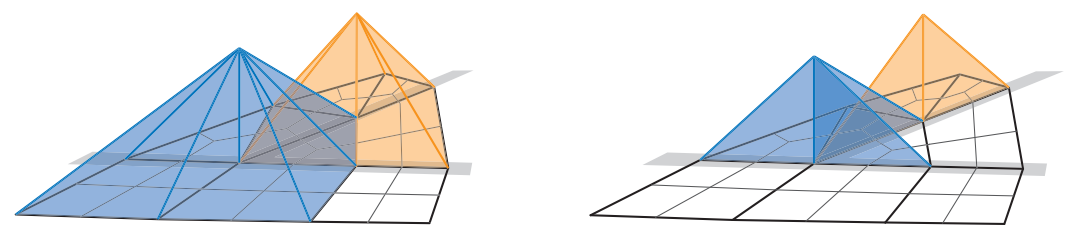

Figure 3.4.: Basis functions for volumetric elements and fracture elements.

\subsubsection{The Approximation Spaces}

For the discretization we introduce the standard conforming, piecewise linear finite element spaces in the matrix and fracture domain

$$
\begin{aligned}
V_{h}^{m} & =\left\{v \in C^{0}\left(\overline{\Omega^{m}}\right) \mid v \text { is linear on } \bar{\Omega}_{e} \in E_{h}^{m}\right\}, \\
V_{h}^{f} & =\left\{v \in C^{0}\left(\overline{\Omega^{f}}\right) \mid v \text { is linear on } \bar{\Omega}_{e} \in E_{h}^{f}\right\},
\end{aligned}
$$

and the non-conforming test space $W_{h}$ (based on the secondary mesh)

$$
\begin{aligned}
& W_{h}=\left\{w \in \mathrm{L}^{2}\left(\Omega^{m}\right) \mid w \text { is constant on each } b_{i}^{m} \in B_{h}^{m}\right\}, \\
& W_{h}^{f}=\left\{w \in \mathrm{L}^{2}\left(\Omega^{f}\right) \mid w \text { is constant on each } b_{i}^{f} \in B_{h}^{f}\right\} .
\end{aligned}
$$

Figure 3.4 shows two basis functions for $V_{h}^{m}$ and $V_{h}^{f}$. Depending on the element type, the term linear has to be replaced by multi-linear in (3.30), 3.3I) as well as in the following (e.g. for quadrilaterals).

We will only describe the treatment of homogeneous Dirichlet-type boundary conditions to keep the presentation free from notational abundance. The subspaces for homogeneous Dirichlet boundary conditions for fracture and matrix and the both phases $\alpha=g, w$ are

$$
\begin{aligned}
V_{h \alpha 0}^{\tau} & =\left\{v \in V_{h}^{\tau}|v|_{\Gamma_{\alpha d}}=0\right\}, \\
W_{h \alpha 0}^{\tau} & =\left\{w \in W_{h}^{\tau}|w|_{\Gamma_{\alpha d}}=0\right\},
\end{aligned}
$$

with $\tau=m$, f. General Dirichlet boundary conditions can be treated as in Bastian (I999). In the case of inhomogeneous Dirichlet boundary conditions it is necessary to employ separate function spaces for water pressure and gas saturation, which adhere to the respective boundary conditions. These spaces can also depend on time, a feature which is also not treated here. This is only a notational convenience and at all times $V_{h \alpha 0}^{\tau}$ and $W_{h \alpha 0}^{\tau}$ should be thought of as $V_{h \alpha 0}^{\tau}(t)$ and $W_{h \alpha 0}^{\tau}(t)$.

The phase saturations $S_{n}$ and $S_{w}$ are a discontinuous quantity at interfaces between media with different properties as well as at all vertices $v_{i} \in \Omega_{h}^{f}$, because these vertices are shared by the rock matrix and the fracture network. A discontinuous saturation can not be represented by the standard conforming finite element spaces $V_{h}^{m}$ and $V_{h}^{f}$ so instead we choose discontinuous saturation spaces

$$
\begin{aligned}
S_{h}^{m} & =\left\{v \in \mathrm{L}^{2}\left(\Omega^{m}\right)|v|_{\Omega_{e}} \text { linear for } \Omega_{e} \in \mathrm{E}_{h}^{m}\right\}, \\
S_{h}^{f} & =\left\{v \in \mathrm{L}^{2}\left(\Omega^{f}\right)|v|_{\Omega_{e}} \text { linear for } \Omega_{e} \in E_{h}^{f}\right\} .
\end{aligned}
$$




\subsection{The Finite Volume Method}

By means of the mappings $\Pi^{m}$ and $\Pi^{f}$,

$$
\begin{aligned}
\Pi^{m}: V_{h}^{m} & \rightarrow S_{h}^{m}, \\
\Pi^{f}: V_{h}^{f} & \rightarrow S_{h}^{f},
\end{aligned}
$$

it is possible to formulate the discretization by the conforming finite element functions from the previous section, but to employ the correct discontinuous saturation function wherever appropriate. These mappings employ the extended interface conditions from section 2.4.6.

$S_{h}^{m}$ is only continuous within elements so we define the mapping for a given $x \in \Omega_{e}$. The values of the function

$$
s_{h}^{m}=\Pi^{m} v_{h} \quad v_{h} \in V_{h}^{m}, s_{h} \in S_{h}^{m}
$$

are uniquely determined by the values of $s_{h}$ in the corners of $\Omega_{e}$ by

$$
s_{h}(x)=\Pi^{m} v_{h}(x)=\sum_{i \in V(e)} S_{i}^{e} \varphi_{i}^{m}(x)
$$

where $V(e)$ is the set of indices of the corner vertices of $\Omega_{e}$ and the value $S_{i}^{e}$ at corner vertex $v_{i}$ is found by

$$
S_{i}^{e}= \begin{cases}v\left(\boldsymbol{x}_{i}\right) & \text { if } p_{c}\left(\boldsymbol{x}^{e}, 1-v\left(\boldsymbol{x}_{i}\right)\right)=p_{c \min }\left(\boldsymbol{x}_{i}\right), \\ 0 & \text { if } p_{c \min }\left(\boldsymbol{x}_{i}\right)<p_{c}\left(\boldsymbol{x}^{e}, 1\right), \\ 1-S & \text { else, with } S \text { from } \boldsymbol{p}_{\mathrm{c}}\left(\boldsymbol{x}^{e}, S\right)=p_{c \min }\left(\boldsymbol{x}_{i}\right) .\end{cases}
$$

Here we employ the minimal capillary pressure function $p_{\text {cmin }}(\boldsymbol{x})$ defined as

$$
p_{\mathrm{cmin}}(x)=\min _{\Omega_{\mathrm{e}} \in \mathrm{E}(\boldsymbol{x})} p_{\mathrm{c}}\left(\boldsymbol{x}^{e}, 1-v(x)\right) .
$$

$E(x)$ is the set of elements which contain $x$ in their closure,

$$
\mathrm{E}(\boldsymbol{x})=\left\{\Omega_{e} \in \Omega^{\mathrm{m}} \cup \Omega^{f} \mid x \in \overline{\Omega_{e}}\right\} .
$$

For fractures the same construction is employed, only that the fracture basis functions are used instead of matrix basis functions,

$$
s_{h}(x)=\Pi^{f} v_{h}(x)=\sum_{i \in V(e)} S_{i}^{e} \varphi_{i}^{f}(x) \quad \text { for } s_{h} \in S_{h}^{f}, v_{h} \in V_{h}^{f}
$$

The connection between the spaces $V_{h}^{m}$ and $V_{h}^{f}$ is created by the projection $\Lambda$. So far, $V_{h}^{m}$ and $V_{h}^{f}$ have been treated as separate spaces with separate unknowns in the vertices of the grid. The projection

$$
\begin{aligned}
& \Lambda: V_{h}^{m} \rightarrow V_{h}^{f} \\
& v_{h}^{m} \mapsto v_{h}^{f} \quad \text { with } v_{h}^{m}(x)=v_{h}^{f}(x) \text { for all } x \in \Omega^{f} .
\end{aligned}
$$

is the function which maps the finite element function $v_{h}^{m}$ defined in the matrix to the finite element function $v_{\mathrm{h}}^{\mathrm{f}}$ defined in the fracture network whose values coincide in the fracture network. Figure 3.5 shows a function $v_{h} \in V_{h}$ and the mappings $\Pi^{m} v_{h}$ into the matrix space and $\Pi^{m} \wedge v_{h}$ into the fracture space. 


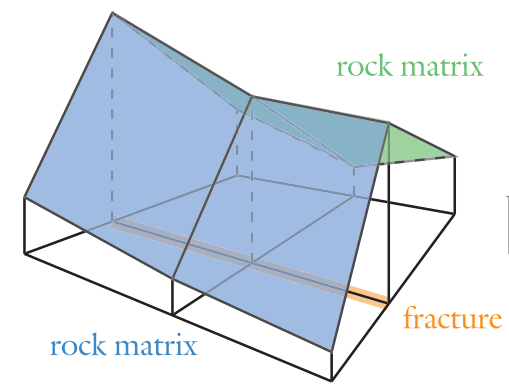

$v_{\mathrm{h}} \in \mathrm{V}_{\mathrm{h}}$

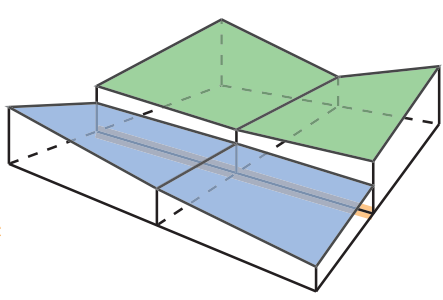

$\Pi^{\mathrm{m}} v_{\mathrm{h}}$

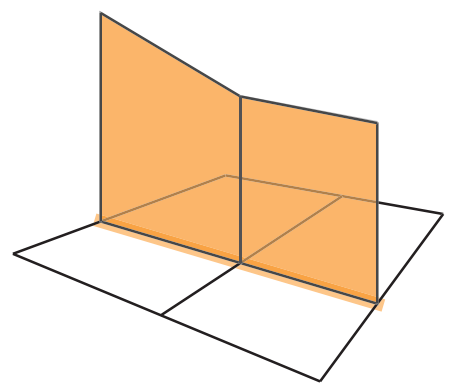

$\Pi^{\mathrm{f}} \wedge v_{\mathrm{h}}$

Figure 3.5.: A function $v_{\mathrm{h}} \in V_{\mathrm{h}}$ and the mapping into the saturation spaces, $\Pi^{\mathrm{m}} v_{\mathrm{h}}$ and $\Pi^{f} \wedge v_{h}$.

\subsubsection{WEAK FORMULATION}

The weak formulation of equations $3 . \mathrm{I8}$ ) for the rock matrix is found by multiplying with the test functions and integration by parts. The resulting weak formulation of the two-phase flow problem in the matrix is (we suppress the time dependencies for notational convenience, and write $p_{w h}, S_{g h}$ for $\left.p_{w h}(t), S_{g h}(t)\right)$ :

Find $\mathrm{p}_{w \mathrm{~h}} \in \mathrm{V}_{\mathrm{hw0}}$ and $\mathrm{S}_{\mathrm{gh}} \in \mathrm{V}_{\mathrm{hg} 0}$ such that for all $\mathrm{w}_{\mathrm{gh}}^{\mathrm{m}} \in \mathrm{W}_{\mathrm{hg} 0}^{\mathrm{m}}$, $w_{w h}^{\mathrm{m}} \in \mathrm{W}_{\mathrm{hw0}}^{\mathrm{m}}$ and $0<\mathrm{t}<\mathrm{T}$

$$
\begin{array}{r}
\frac{\partial}{\partial t} \sum_{b \in B_{h}^{m}} \int_{b} \Phi \rho_{w}\left(1-\Pi^{m} S_{g h}\right) d x+\sum_{\gamma \in \Gamma_{\text {int }}^{m}} \int_{w} \rho_{w} v_{w} \cdot \mathfrak{n}\left[w_{w h}^{m}\right] d s \\
+\sum_{\gamma \in \Gamma_{\text {ext }}^{m} \cap \Gamma_{w n}} \int_{\gamma} \phi_{w} w_{w h}^{m} d s-\sum_{b \in B_{h}^{m}} \int_{b} \rho_{w} q_{w} d x=0 \\
\frac{\partial}{\partial t} \sum_{b \in B_{h}^{m}} \int_{b} \Phi \rho_{g} \Pi^{m} S_{g h} d x+\sum_{\gamma \in \Gamma_{\text {int }}^{m}} \int_{\gamma} \rho_{g} v_{g} \cdot \mathfrak{n}\left[w_{g h}^{m}\right] d s \\
+\sum_{\gamma \in \Gamma_{\text {ext }}^{m} \cap \Gamma_{g n}} \int_{\gamma} \phi_{g} w_{g h}^{m} d s-\sum_{b \in B_{h}^{m}} \int_{b} \rho_{g} q_{g} d x=0
\end{array}
$$

In the equations the first term is called the accumulation term, the second term is the internal flux term, the third is the boundary flux term and the fourth the source and sink term. For the numerical evaluation of the accumulation term we employ a midpoint rule, which corresponds to the mass lumping approach in the finite element method. The Darcy velocities in the interior flux terms are evaluated with an upwind scheme. For the water phase this is for a given side $\gamma=\gamma_{e, b, b^{\prime}}$

$$
\int_{\gamma} \rho_{w} \boldsymbol{v}_{w} \cdot \mathbf{n}\left[w_{h}\right] \mathrm{d} s=\int_{\gamma} \rho_{w} \lambda_{w \gamma}^{\star} \tilde{\boldsymbol{v}}_{w} \cdot \mathbf{n}\left[w_{h}\right] \mathrm{ds}
$$




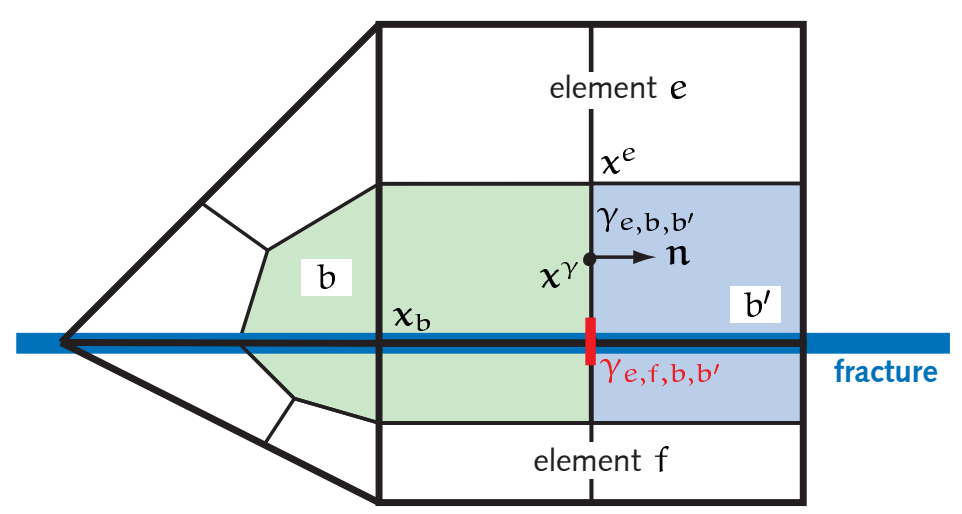

Figure 3.6.: Notation for control volumes

with the upwind evaluation of the mobility

$$
\lambda_{w \gamma}^{\star}=(1-\beta) \lambda_{w h}\left(\boldsymbol{x}^{\gamma}\right)+\beta \cdot \begin{cases}\lambda_{w h}\left(\boldsymbol{x}_{\mathrm{b}}\right) & \text { if } \tilde{\boldsymbol{v}}_{w} \cdot \mathfrak{n} \geq 0 \\ \lambda_{w h}\left(\boldsymbol{x}_{\mathrm{b}^{\prime}}\right) & \text { else }\end{cases}
$$

and the directional part of the velocity

$$
\tilde{\boldsymbol{v}}_{w}=-\mathrm{K}\left(\boldsymbol{x}^{e}\right)\left(\nabla p\left(\boldsymbol{x}^{\gamma}\right)-\rho_{w}\left(\boldsymbol{x}^{\gamma}\right) \mathbf{g}\right)
$$

$x^{\gamma}$ is the center of $\gamma$ and $x_{b}$ is the grid vertex inside control volume $b$. The source and sink terms and the boundary flux terms are evaluated by the midpoint rule. The same evaluation scheme is employed for the gas phase saturation. The parameter $\beta$ controls the upwinding strategy. For $\beta=1$ fully upwinding is achieved, while $\beta=0$ results in a central differencing scheme. We employ a fixed $\beta$, but adaptive choices depending on the local Peclet number are possible (Michev, I996). There is also the possibility to choose fixed, but different $\beta$ for fracture and matrix discretization, akin to the assumption that the flow processes in the matrix are not convection dominated opposed to the fractures. The full-upwinding scheme is known to introduce grid-dependencies into the solution, which disappear if sufficiently fine grids are employed. Unfortunately, this resolution is extremely small for gas-water problems and some numerical artifacts must be expected.

The forms defined in 3.47 can be abbreviated as the vector-valued form

$$
m^{m}\left(p_{w h}, S_{g h}, w_{w h}^{m}, w_{g h}^{m}\right)+a^{m}\left(p_{w h}, S_{g h}, w_{w h}^{m}, w_{g h}^{m}\right)=0
$$

where we collect the accumulation terms in $\mathrm{m}^{\mathrm{m}}(\cdot, \cdot, \cdot, \cdot)$ and the internal and boundary flux terms and source and sink terms in $a^{m}(\cdot, \cdot, \cdot, \cdot)$. Note that both $w_{w h}^{m}$ and $w_{g h}^{m}$ are only necessary if Dirichlet boundary conditions are present in different parts of the boundary for $p_{w}$ and $S_{g}$.

The corresponding form for the fracture space $\mathrm{m}^{f}(\cdot, \cdot, \cdot, \cdot)+\mathrm{a}^{\mathrm{f}}(\cdot, \cdot, \cdot, \cdot$,$) is derived by replac-$ ing superscript $m$ with $f$ and not using $p_{w}$ and $S_{g}$ directly in $m^{f}(\cdot, \cdot, \cdot, \cdot)+a^{f}(\cdot, \cdot, \cdot, \cdot)$, but 


\section{Finite Volume Discretization and Multigrid Solution}

using their projections to the fracture space. The weak formulation is: Find $p_{w h} \in V_{h w 0}$ and $\mathrm{S}_{\mathrm{gh}} \in \mathrm{V}_{\mathrm{hg} 0}$ such that for all $w_{\mathrm{gh}}^{\mathrm{f}} \in \mathrm{W}_{\mathrm{hg} 0}^{\mathrm{f}}$, $w_{w h}^{\mathrm{f}} \in \mathrm{W}_{\mathrm{hw} \text { o }}^{\mathrm{f}}$ and $0<\mathrm{t}<\mathrm{T}$

$$
m^{f}\left(\Lambda p_{w h}, \wedge S_{g h}, w_{w h}^{f}, w_{g h}^{f}\right)+a^{f}\left(\Lambda p_{w h}, \Lambda S_{g h}, w_{w h}^{f}, w_{g h}^{f}\right)=0 .
$$

Note that $w_{w h}^{f}$ and $w_{g h}^{f}$ are just a notational convenience: The fracture space test functions are related to the matrix space test functions by the projection $\Xi: w_{\alpha h}^{m} \mapsto w_{\alpha h}^{f}$.

The final formulation of the coupled scheme is now found by adding the two forms: Find $p_{w h} \in V_{h w 0}$ and $S_{g h} \in V_{h g 0}$ such that for all $w_{\mathrm{gh}} \in \mathrm{W}_{\mathrm{hg} 0}^{\mathrm{m}}, w_{w h} \in \mathrm{W}_{\mathrm{hw0}}^{\mathrm{m}}$ and $0<\mathrm{t}<\mathrm{T}$

$$
\mathrm{m}\left(\mathrm{p}_{w h}, \mathrm{~S}_{\mathrm{gh}}, w_{w h}, w_{\mathrm{gh}}\right)+\mathrm{a}\left(\Lambda \mathrm{p}_{w h}, \wedge S_{\mathrm{gh}}, \Xi w_{w h}, \Xi w_{g h}\right)=0
$$

where $\mathrm{m}(\cdot, \cdot, \cdot, \cdot)=\mathrm{m}^{\mathrm{m}}(\cdot, \cdot, \cdot, \cdot \cdot)+\mathrm{m}^{f}(\cdot, \cdot, \cdot, \cdot)$ and $a(\cdot, \cdot, \cdot, \cdot)=a^{\mathrm{m}}(\cdot, \cdot, \cdot, \cdot)+\mathrm{a}^{\mathrm{f}}(\cdot, \cdot, \cdot, \cdot, \cdot)$.

\subsubsection{IMPLEMENTATION}

The implementations of finite volume codes often use a loop over all elements and calculate the contribution of the dual grid skeleton from inside each element. The implementation of the presented method for rock matrix and fractures can be done based on a volumetric element code without the need to introduce the notion of lower-dimensional elements into the code, if fractures are represented as inner boundaries and if each element calculates not only the contributions from $\Gamma_{\text {int }}^{\mathrm{m}}$, but also from $\Gamma_{\text {int }}^{f}$. Calculation of the contribution to the stiffness matrix and the defect from $\gamma_{e, f, b, b^{\prime}}$ is done by element $\Omega_{e}$ and $\Omega_{f}$ which each contribute half of the value. This approach is advantageous, because it stays within the element-wise implementation paradigm and doesn't require boundary-communications if the method is implemented on parallel computers with a domain decomposition approach.

\subsection{Time Discretization}

The traditional approach to the numerical solution of time-dependent partial differential equations is by the method of lines. First, a spatial discretization is applied to the problem (e. g. a finite element method or a finite volume method), which leads to a system of ordinary differential equations. This system is then solved by a time differencing scheme which can be chosen from the wide range of available methods, see Hairer and Wanner (I996), Thomée (I997).

The opposite approach is taken by the Rothe method, which first applies a time differencing scheme and then approaches the resulting problem by a spatial discretization. The advantage of this method is that different spatial discretizations can be applied during the time stepping process.

In the context of finite element methods and finite volume methods we have to deal with stiff systems of ordinary differential equations. The first definition of the term stiff was rather pragmatic: "stiff equations are equations where certain implicit methods, in particular BDF, perform better, usually tremendously better, than explicit ones" (Curtiss and Hirschfelder, I952). We will indeed focus our attention on implicit methods. 


\subsection{Time DisCRETIZATION}

We divide the time interval $(0, T)$ into discrete time steps

$$
0=t^{0}, \ldots, t^{k}, t^{k+1}=t^{k}+\Delta t^{k}, \ldots, t^{M}=T
$$

of variable or fixed size. Superscript $n$ notation

$$
p_{w h}\left(t^{n}\right)=p_{w h}^{n} \quad \text { and } \quad S_{g h}\left(t^{n}\right)=S_{g h}^{n}
$$

for functions and coefficient vectors denotes values at time step $t^{n}$. The coefficient vectors $v \in \mathbb{R}^{N}$ are related to the discrete solution function $v_{h} \in V_{h}$ by the mapping $\mathcal{C}: \mathbb{R}^{N} \rightarrow V_{h}$ in this way:

$$
\mathcal{C}(\boldsymbol{v})=v_{\mathrm{h}}, \quad v_{\mathrm{h}}(\boldsymbol{x})=\sum_{\mathrm{i} \in \mathrm{I}} v_{\mathrm{i}} \varphi_{\mathrm{i}}(\boldsymbol{x}) .
$$

The application of the finite volume discretization scheme leads to the semi-discretization

$$
\begin{gathered}
\frac{\partial}{\partial t} \mathbf{M}_{w}\left(\mathbf{p}_{w}(t), \mathbf{S}_{g}(t)\right)+\mathbf{A}_{w}\left(\mathbf{p}_{w}(t), \mathbf{S}_{g}(t)\right)=0, \\
\frac{\partial}{\partial t} \mathbf{M}_{g}\left(\mathbf{p}_{w}(t), \mathbf{S}_{g}(t)\right)+\mathbf{A}_{g}\left(\mathbf{p}_{w}(t), \mathbf{S}_{g}(t)\right)=0,
\end{gathered}
$$

where $\mathbf{M}$ corresponds to $\mathrm{m}$ and $\boldsymbol{A}$ corresponds to $a$. The system can be written as

$$
\left(\begin{array}{ll}
\mathbf{M}_{w w} & \mathbf{M}_{w g} \\
\mathbf{M}_{g w} & \mathbf{M}_{g g}
\end{array}\right)\left(\begin{array}{l}
\frac{\partial \mathbf{p}_{w}(t)}{\partial t} \\
\frac{\partial \mathbf{S}_{g}(t)}{\partial t}
\end{array}\right)+\left(\begin{array}{l}
\mathbf{A}_{w}\left(\mathbf{p}_{w}, \mathbf{S}_{g}\right) \\
\boldsymbol{A}_{\mathfrak{n}}\left(\mathbf{p}_{w}, \mathbf{S}_{g}\right)
\end{array}\right)=0
$$

with the submatrices

$$
\left(\mathbf{M}_{\alpha w}\right)_{i j}=\frac{\partial \mathbf{M}_{\alpha w, i}}{\partial \mathbf{p}_{w, j}} \quad\left(\mathbf{M}_{\alpha g}\right)_{i j}=\frac{\partial \mathbf{M}_{\alpha \mathrm{g}, \mathrm{i}}}{\partial \mathbf{S}_{\mathrm{g}, \mathrm{j}}} .
$$

This results in a system of differential algebraic equations (DAE) of index 1 in implicit form. The matrix $\mathbf{M}$,

$$
\mathbf{M}=\left(\begin{array}{ll}
\mathbf{M}_{w w} & \mathbf{M}_{w g} \\
\mathbf{M}_{g w} & \mathbf{M}_{g g}
\end{array}\right)
$$

is singular in the incompressible case. An analysis for the incompressible case shows that a discrete form of the elliptic equation has to be satisfied. This is called the implicit constraint. It is shown, that a backward Euler step guarantees the validity of the implicit constraint. Further time-steps can be chosen from a different method-the constraint is satisfied if it has been satisfied on the previous time level. For this reason, we always employ one backward Euler step as the first time step, regardless of the time differencing scheme of the subsequent steps.

The time step scheme reads in the one-step- $\theta$ notation: For $n=0,1, \ldots, M-1$ find $\mathbf{p}_{w}^{\mathfrak{n}}, \mathbf{S}_{\mathfrak{n}}^{n}$ such that

$$
\mathbf{M}_{w}^{\mathrm{n}+1}-\mathbf{M}_{w}^{\mathrm{n}}+\Delta \mathrm{t}^{\mathrm{n}} \theta \boldsymbol{A}_{w}^{\mathrm{n}+1}+\Delta \mathrm{t}^{\mathrm{n}}(1-\theta) \boldsymbol{A}_{w}^{\mathrm{n}}=0
$$




\section{Finite Volume Discretization and Multigrid Solution}

$$
\mathbf{M}_{\mathrm{g}}^{\mathrm{n}+1}-\mathbf{M}_{\mathrm{g}}^{\mathrm{n}}+\Delta \mathrm{t}^{\mathrm{n}} \theta \boldsymbol{A}_{\mathrm{g}}^{\mathrm{n}+1}+\Delta \mathrm{t}^{\mathrm{n}}(1-\theta) \mathbf{A}_{\mathrm{g}}^{\mathrm{n}}=0 .
$$

For $\theta=0$ this is the forward Euler scheme or explicit Euler scheme, for $\theta=1 / 2$ the CrankNicholson scheme, and for $\theta=1$ the backward Euler scheme or implicit Euler scheme,

The explicit Euler scheme is first-order accurate, but subject to severe time step size restrictions, so we don't consider this or other explicit schemes any further. The implicit Euler scheme is first-order accurate and strongly A-stable, the Crank-Nicholson scheme is second order accurate and A-stable. Stability is defined by considering the Dahlquist equation $y^{\prime}=\lambda y, y(0)=1$, and then deriving the stability function $R(z)$ for the calculation rule

$$
y^{k+1}=R(h \lambda) y^{k} .
$$

$\mathrm{R}$ is a polynomial for explicit methods and a rational function for implicit methods. The stability domain of a method with stability function $\mathrm{R}(z)$ is

$$
S=\{z \in \mathbb{C}|| R(z) \mid \leq 1\}
$$

A method is called $A$-stable if its stability domain satisfies

$$
\mathbb{C}^{-} \subset S, \quad \mathbb{C}^{-}=\{z \mid \operatorname{Re} z \leq 0\}
$$

If $|\mathrm{R}(\infty)|<1$ the method is called strongly $A$-stable. The one-step- $\theta$ scheme has the stability function

$$
\left.R(-\lambda)=\frac{1-\left(\theta-\frac{1}{2}\right) \lambda}{1+\theta \lambda}=e^{-\lambda}+\mathcal{O}\left(\left(\theta-\frac{1}{2}\right)|\lambda|^{2}+|\lambda|^{3}\right)\right), \quad|\lambda| \leq 1 .
$$

A time differencing scheme should possess these properties (Rannacher, I999):

A-stability to ensure local convergence

$$
|R(-\lambda)| \leq 1
$$

Global stability to ensure global convergence

$$
\varlimsup_{\operatorname{Re} \lambda \rightarrow \infty}|R(-\lambda)| \leq 1-\mathcal{O}\left(\Delta t^{k}\right)
$$

Strong A-stability to ensure the smoothing property

$$
\varlimsup_{\operatorname{Re} \lambda \rightarrow \infty}|R(-\lambda)| \leq 1-\delta<0
$$

Low dissipation to ensure energy preservation

$$
|R(-\lambda)|=1-\mathcal{O}(\operatorname{Im} \lambda), \quad \text { for } \operatorname{Re} \lambda \rightarrow 0
$$




\subsection{Nonlinear System Solution}

The implicit Euler scheme is very stable and damps out perturbations in the data, but it is also very dissipative and will smear out sharp fronts in a solution. The Crank-Nicholson scheme is far less dissipative, but it lacks the stability of the implicit Euler method towards perturbations in the solution. By adapting the time step size this stability concern can be met, but the necessary time step size may be prohibitively small.

The backward-differencing formulx (BDF) are a family of schemes, including the popular second order $\mathrm{BDF}(2)$ method. But $\mathrm{BDF}(2)$ it is not globally mass conservative in the case of variable time step sizes - a property possessed by the implicit Euler scheme and the CrankNicholson scheme. Also of second order is the singly diagonally implicit Runge-Kutta scheme SDIRK(2) (Alexander, 1977).

The fractional-step- $\theta$ scheme was originally developed as an operator splitting scheme for the incompressible Navier-Stokes equations. It consists of three substeps $t^{n} \rightarrow t^{n+\alpha} \rightarrow$ $t^{n+1-\alpha} \rightarrow t^{n+1}$, where each substep $k$ is a one-step- $\theta$ step with $\theta_{k}$ and $\Delta t^{k}$ chosen as

$$
\begin{array}{ll}
\theta_{1}=2-\sqrt{2} & \Delta t^{1}=(1-\sqrt{2} / 2) \Delta t=\alpha \Delta t, \\
\theta_{2}=\sqrt{2}-1 & \Delta t^{2}=(\sqrt{2}-1) \Delta t=(1-\alpha) \Delta t, \\
\theta_{3}=2-\sqrt{2} & \Delta t^{3}=(1-\sqrt{2} / 2) \Delta t=\alpha \Delta t
\end{array}
$$

The $\theta_{i}$ can be chosen different than above as long as $\theta_{1}=\theta_{3}=\theta \in(1 / 2,1]$, and $\theta_{2}=1-\theta$ holds. The fractional-step- $\theta$ scheme is of second order for $\alpha=1-\sqrt{2} / 2$ and strongly A-stable for any $\theta \in(1 / 2,1]$. Its stability function is

$$
\mathrm{R}(z)=\left(\frac{1+\alpha z(1-\theta)}{1-\alpha z \theta}\right)^{2}\left(\frac{1+(1-2 \alpha) z\left(1-\theta_{2}\right)}{1-(1-2 \alpha) z \theta_{2}}\right)
$$

The scheme possesses, other than the Crank-Nicholson scheme, the full smoothing property in case of rough initial data. Note that the substepping does not result in higher computational cost since the step size $\Delta t$ can be chosen three times larger than for the single-step- $\theta$ scheme.

Finally, an interesting time differencing scheme is the discontinuous Galerkin method (not to be confused with the class of spatial discretization methods by the same name), see Thomée (I997), Eriksson et al. (1996). It is derived by applying a Galerkin finite element method to the time variable, which has the advantage that if Galerkin finite element methods are used for spatial discretization, the resulting method treats time and space variables similarly. The approximate solution in $t$ will be sought from the space of piecewise polynomial functions, which are not necessarily continuous at the nodes.

\subsection{Nonlinear System Solution}

In the fully coupled, fully implicit discretization, a large system of nonlinear equations has to be solved in each time step and an accurate solution of the equations is necessary, because otherwise the local mass conservation could be destroyed. The nonlinear system solution is done with an inexact Newton method, where the linearized systems arising in each step of the Newton method are solved iteratively with a multigrid method. Without exact solution 


\section{Finite Volume Discretization and Multigrid Solution}

of the linearized systems, the quadratic convergence of the Newtons method is reduced, but since quadratic convergence can only be observed in a close neighborhood of the solution, where the error is already much smaller than the discretization error, this is not a disadvantage in practice. To ensure global convergence, a line search algorithm is applied in the Newton steps (Braess, I992).

The discretization scheme leads to a system of nonlinear algebraic equations

$$
\mathbf{F}(z)=0
$$

with a vector $z$ which contains pressure and saturation unknowns,

$$
\boldsymbol{z}=\left(\mathbf{p}_{w, 1}, \ldots, \mathbf{p}_{w, \mathrm{~N}}, \mathbf{S}_{\mathrm{n}, 1}, \ldots, \mathbf{S}_{\mathrm{n}, \mathrm{N}}\right)^{\top}
$$

and a vector function $\mathbf{F}$ with components

$$
\mathbf{F}=\left(\mathbf{F}_{w, 1}, \ldots, \mathbf{F}_{w, N}, \mathbf{F}_{n, 1}, \ldots, \mathbf{F}_{n, N}\right)^{\top}
$$

For the one step $\theta$ scheme the components are given by

$$
\mathbf{F}_{\alpha}=\mathbf{M}_{\alpha}^{\mathrm{n}+1}-\mathbf{M}_{\alpha}^{\mathrm{n}}+\Delta \mathrm{t}^{\mathrm{n}} \theta\left(\boldsymbol{A}_{\alpha}^{\mathrm{n}+1}\right)+\Delta \mathrm{t}^{\mathrm{n}}(1-\theta)\left(\boldsymbol{A}_{\alpha}^{\mathrm{n}}\right) .
$$

Dirichlet values are included in the equation system and treated by inserting a trivial equation.

The linearized equations are described by the Jacobian matrix $\mathbf{J}$ of $\mathbf{F}$ at point $z$,

$$
(\mathbf{J}(\boldsymbol{z}))_{i j}=\frac{\partial \mathbf{F}_{i}}{\partial z_{j}}(\boldsymbol{z})
$$

The entries of $\boldsymbol{A}$ are computed by numerical differentiation,

$$
\frac{\partial \mathbf{J}_{i}}{\partial z_{j}}(z)=\frac{\mathbf{F}_{i}\left(z+\Delta z_{j} \mathbf{e}_{j}\right)-\mathbf{F}_{\mathfrak{i}}(\boldsymbol{z})}{\Delta \boldsymbol{z}_{j}}+(\mathrm{O})\left(\Delta \boldsymbol{z}_{\mathfrak{j}}\right)
$$

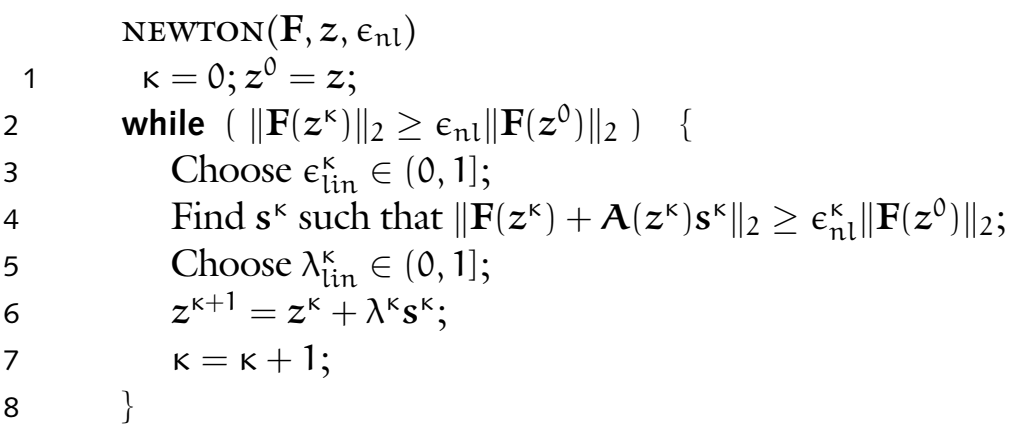

Algorithm $\operatorname{NewTON}\left(\mathbf{F}, z, \epsilon_{\mathfrak{n l}}\right)$ describes the nonlinear systems solution process. In the description, $\|\cdot\|_{2}$ is the Euclidean vector norm. Line 6 of the Newton iteration algorithm 


\subsection{Multigrid}

implements a simple line search strategy (Braess, I992), which helps to achieve global convergence of the Newton method. The damping factor $\lambda^{k}$ is chosen as the maximum of $\left\{1, \frac{1}{2}, \frac{1}{4}, \ldots\right\}$ such that

$$
\left\|\mathbf{F}\left(z^{\kappa}+\lambda^{\kappa} \mathbf{s}^{\kappa}\right)\right\|_{2} \leq\left(1-\frac{1}{4} \lambda^{\kappa}\right)\left\|\mathbf{F}\left(z^{\kappa}\right)\right\|_{2}
$$

The error in the solution of the linearized equations, which is monitored in the forcing term $\epsilon_{\text {lin }}^{K}$, should be sufficiently small because otherwise saturation values can lie outside the interval $[0,1]$.

Initial guesses for the solution can be the solution from the last time step-which usually gives good initial guesses as long as the time step size is not too large-or by a hierarchical strategy, where the nonlinear system is solved on the coarsest mesh, interpolated to the next finer mesh and taken as the initial guess. The process is repeated up to the finest grid level. This is an effective strategy for the first time step, where no previous solution is available. A combination of both approaches is possible and advantageous for large time steps, where the initial solution on level 0 uses the solution from the previous time step as an initial guess.

Nonlinear systems can also be solved by nonlinear multigrid methods where the smoother in the multigrid method (see next section) is replaced by a nonlinear version. We did not apply this method, because it is usually less efficient than multigrid methods combined with the Newton method and because only Jacobi- and Gauß-Seidel type smoothers are available, while for the linear multigrid method more robust smoothers exist.

In the context of this work it is well known that the nonlinear systems resulting from discretization schemes with the extended capillary pressure condition are more difficult to solve than systems from discretization schemes without the extended capillary pressure condition (Jakobs et al., 2003). The solution gets increasingly difficult for smaller viscosities and for steep capillary pressure and relative permeability functions. This situation arises when gas saturation increases at the end of a fracture and has to reach the entry pressure before entering the matrix. Since this configuration is found at many places throughout a fracture network, the convergence of the nonlinear solver is of crucial importance for the overall efficiency.

\subsection{Linear System Solution: The Multigrid Method}

The solution of the linear systems arising in the implicit time discretization is usually the computationally most expensive part of the numerical solution of partial differential equations. In the present setting, the linear system

$$
A x=b, \quad A \in \mathbb{R}^{N \times N}, b, x \in \mathbb{R}^{N}
$$

arises from the fully coupled Newton solution of the nonlinear equations, where $A$ is the Jacobian and $b$ the nonlinear defect. Direct solution of $(3.7 \mathrm{I})$ requires $\mathcal{O}\left(\mathrm{N}^{3}\right)$ arithmetical operations (Golub and van Loan, I996), which can be reduced by banded elimination to $\mathcal{O}\left(\mathrm{N}^{2}\right)$ or by nested dissection to $\mathcal{O}\left(\mathrm{N}^{1.5}\right)$ for two-dimensional problems arising from the discretization of partial differential equations. This makes the solution of equations with some 


\section{Finite Volume Discretization and Multigrid Solution}

hundred thousand unknowns possible. In three space dimension, the operations can not be reduced so substantially and $\mathcal{O}\left(\mathrm{N}^{2.33}\right)$ or $\mathcal{O}\left(\mathrm{N}^{2}\right)$ operations for the mentioned techniques remain. This complexity is by far too large for the considered complex three-dimensional systems where several million unknowns must be expected.

A more efficient solution method for systems of linear equations is offered by iterative schemes. We consider relaxation methods, where the matrix $A$ is split into two matrices $M$ and $\mathrm{N}$,

$$
A=M-N .
$$

$M$ is chosen as an approximation to $A$ which is easy to invert. From a given initial guess $x^{0}$, a sequence of iterations is computed by

$$
x^{k+1}=\mathbf{b}^{k}+M^{-1} d^{k}, \quad d^{k}=\left(b-A x^{k}\right) .
$$

$\mathrm{d}^{\mathrm{k}}$ is the defect in step $\mathrm{k}$. Choices of $M$ which allow for an easy inversion are the diagonal of $A$, which gives the Jacobi method, and the lower triangle of $A$, for which the Gauß-Seidel method results. The symmetric Gauß-Seidel method consists of one forward step of the GaußSeidel method (i. e. with the lower triangle of $A$ ) followed by a backward step of GaußSeidel (i. e. with the upper triangle of A). Another popular method is the incomplete LU factorization (ILU), where

$$
M=\mathrm{LU}
$$

is the $\mathrm{LU}$ factorization of $A$ in which the sparsity pattern of $A$ is kept for $L$ and $U$, in order to avoid the fill-in which could lead to dense matrices - this would not only jeopardize the efficiency of the method but also the memory requirements of the algorithm.

The convergence of a method can be measured by the factor $\rho$

$$
\left\|x-x^{k+1}\right\| \leq \rho\left\|x-x^{k}\right\|
$$

with a suitable norm $\|\cdot\|$. A method is convergent if and only if for the matrix

$$
\mathrm{B}=\mathrm{I}-\mathrm{M}^{-1} \mathrm{~A}=\mathrm{M}^{-1} \mathrm{~N}
$$

the spectral radius of $\mathrm{B}$ of is smaller than one,

$$
\rho(B)<1 .
$$

Reducing the error by a factor $\epsilon$ requires at most $\lceil\log \epsilon / \log \rho\rceil$ iterations. For these results and an excellent overview of iterative methods see the monograph by Hackbusch (1994).

For systems arising from the discretization of partial differential equations on a grid with mesh size $h$, a convergence factor of

$$
\rho=1-\mathcal{O}\left(h^{2}\right)
$$

is common. By the successive overrelaxation method (SOR), this can be enhanced to $\rho=$ $1-\mathcal{O}(\mathrm{h})$, but the SOR method relies on a problem-dependent parameter which is normally 


\subsection{Multigrid}

not known. Convergence of the form $(3.78)$ means that halving the mesh size $h$ leads to a fourfold increase in the necessary number of iterations.

Convergence theory for the presented relaxation methods is available for many classes of matrices, including positive definite matrices, diagonally dominant matrices and $\mathrm{M}$-matrices (Hackbusch, I994). However, the Jacobian arising in the solution of the multiphase flow equations as presented above does not fall into any of these classes.

A very efficient class of iterative schemes are Krylov subspace methods. These methods look for optimal approximations of $x-x^{0}$ in the m-dimensional Krylov subspace $K_{m}$,

$$
K_{m}\left(A, x^{0}\right)=\operatorname{span}\left\{x^{0}, A x^{0}, A^{2} x^{0}, \ldots, A^{m-1} x^{0}\right\} .
$$

Introductions to Krylov subspace methods can be found in Hackbusch (1994), Barrett et al. (1994), and Golub and van Loan (1996). There are many ways to specify optimal approximations, and consequently there is a large range of methods based on the Krylov subspace principle. Among the most popular are the conjugate gradient method (CG method), the generalized conjugate residuals method (GCR method), the generalized minimal residual method (GMRES), and the bi-conjugate gradient stabilized method (Bi-CGSTAB). Of these methods, Bi-CGSTAB and GMRES are especially popular in fluid mechanics. For general matrices $A$, Bi-CGSTAB is less robust than GMRES, but in practice Bi-CGSTAB is often much more efficient because it is cheaper in each step. Bi-CGSTAB also requires less memory than GMRES. In this work we only use Bi-CGSTAB out of the available Krylov subspace methods.

Krylov subspace methods benefit greatly from preconditioning, either by one of the relaxation schemes mentioned above (ILU is a popular choice), or by the multigrid method discussed below.

Multigrid methods are a very important class of iterative methods. Their impact has been so large that their discovery is the most significant development in numerical analysis in the last 25 years' (Wesseling, 200I). Among the many introductions and overviews to the subject we mention Hackbusch (I985), Wesseling (I992), Bramble (I993), and Briggs et al. (2000). Multigrid methods are based on the observation that relaxation methods applied to the model equation $-\Delta u=f$ damp high oscillatory errors more effectively than the low frequency error components. A coarse grid correction step in the multigrid method reduces these low frequency errors and leads to a very efficient scheme.

The multigrid method is of optimal order $\mathcal{O}(\mathrm{N})$ for elliptic model problems and the convergence factor $\rho$ is independent of the mesh size. There is no other method which achieves the same convergence rate. The convergence theory for elliptic problems with full regularity assumptions had reached a mature status by the mid I980s, culminating in the book of Hackbusch (1985). Further developments included regularity perturbations into the theory (Xu, I992, Bramble, I993) and led to convergence proofs for complex problems like the Stokes equations (Wittum, I990, Verfürth, I988). Multigrid methods have been used for the solution of two-phase flow problems in an IMPES formulation (Scott, 1985, Dendy Jr., 1987), but there the multigrid method was only applied to the scalar elliptic pressure equation. Brakhagen and Fogwell (I990) and Molenaar (I995) applied multigrid methods to the fully implicit, fully coupled formulation, but restricted themselves to the incompressible case 


\section{Finite Volume Discretization and Multigrid Solution}

on structured meshes in two space dimensions. The first application of multigrid to the fully implicit, fully coupled approach on general meshes in two and three space dimensions was done by Bastian (I999), Bastian and Helmig (I999).

\subsection{The Multigrid Algorithm}

The multigrid algorithm operates on a sequence of meshes

$$
E_{0}, E_{1}, \ldots, E_{J}
$$

which are generated from the initial coarse mesh $E_{0}$ by uniform or adaptive refinement. For the refinement we employ the stable grid refinement algorithm of Lang (2000) (see also Bastian (1996) and Bey (1998)), which creates grids without hanging nodes.

On each grid level, the discretized equations are now given by

$$
A_{l} x_{l}=b_{l}, \quad l=0, \ldots, J, \quad A \in \mathbb{R}^{N_{l} \times N_{l}}, b, x \in \mathbb{R}^{N_{l}} .
$$

A restriction operator $\mathrm{R}$ and a prolongation operator $\mathrm{P}$ are responsible for the grid transfer between the different grid levels,

$$
\begin{aligned}
& R_{l}: \mathbb{R}^{N_{l}} \rightarrow \mathbb{R}^{N_{l-1},} \\
& R_{l}: \mathbb{R}^{N_{l-1}} \rightarrow \mathbb{R}^{N_{l}} .
\end{aligned}
$$

For conforming finite element methods the prolongation can be defined by finite element interpolation,

$$
\left(P_{l} c_{l-1}\right)=\sum_{j=1}^{N_{l-1}} c_{l-1, j} \varphi_{l-1, j}\left(x_{l-1, i}\right) .
$$

Here $\varphi_{l-1, j}$ is the finite element basis function corresponding to vertex $x_{l, j}$ on level $j . P_{l}$ is a sparse matrix (due to the local support of the basis functions) of rectangular form. The restriction matrix $R_{l}$ is chosen as

$$
\mathrm{R}_{\mathrm{l}}=\mathrm{P}_{\mathrm{l}}^{\top}
$$

On each grid level a relaxation scheme can be applied; in the context of multigrid methods it is referred to as a smoother, due to is effect on the high oscillatory error components.

The coarse grid matrices $A_{l}$ can either be constructed by discretization of the continuous problem on the individual grids (the approach taken in this work), or by the Galerkin coarse grid operator approach

$$
A_{l-1}=R_{l} A_{l} P_{l} .
$$

The parameter $\gamma$ in line 9 of the multigrid algorithm mgc determines the cycle form, for $\gamma=1$ the $\mathrm{V}$-cycle and for $\gamma=2$ the $\mathrm{W}$-cycle results. The parameters $v_{1}, v_{2}$ determine the number of pre- and post-smoothing steps. 


\subsection{Multigrid}

Algorithm 1: The standard multigrid algorithm for one iteration from the finest level $l$ applied to $x_{l}$ with right hand side $\mathbf{b}_{l}$.

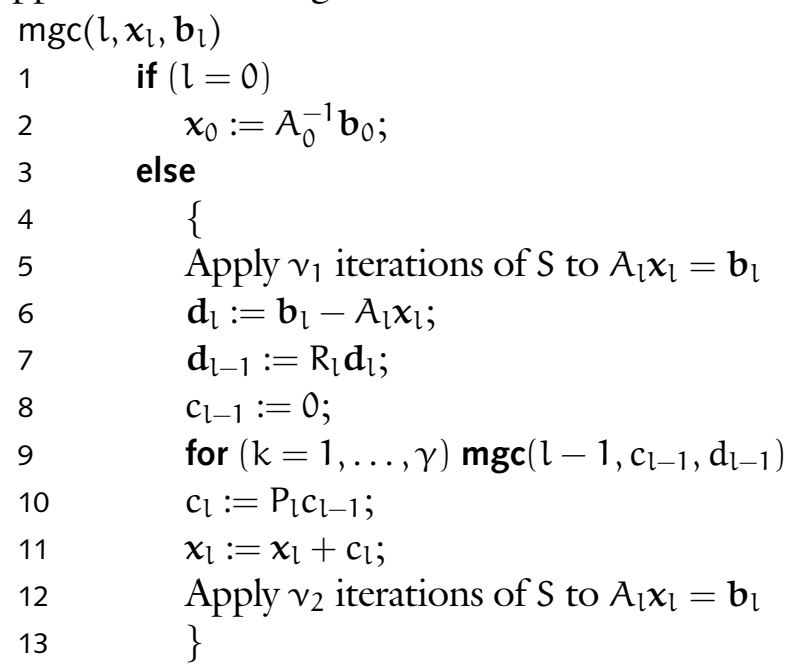

\subsubsection{Robust Multigrid Algorithis}

A multigrid algorithm is considered robust if its convergence rate does not depend on the coefficients of the discretized partial differential equation. In practice, difficulties with multigrid convergence usually arises from interface problems, anisotropic problems or convection dominated convection-diffusion problems. We comment on the types of problems and the solution approaches that have been developed to overcome these difficulties.

INTERFACE PROBLEMS can be studied with the groundwater equation

$$
-\nabla \cdot(K \nabla u)=f .
$$

If the coefficient $\mathrm{K}=\mathrm{K}(\boldsymbol{x})$ is smooth or even constant across the domain, multigrid converges with a convergence rate independent of $h$. But for many problems, the variation in $K$ is not smooth and instead exhibits discontinuities of several orders of magnitude (e. g. between a fracture and the rock matrix). In this case we can distinguish between discontinuities which are resolved by the coarse grid and discontinuities which arise inside elements. In the first case, multigrid converges well and it is possible to prove almost optimal convergence in the two-dimensional case (Bramble et al., I991), but in three space dimensions coefficient distributions which deteriorate multigrid convergence are possible (Dryja et al., I996). If the discontinuities are not aligned with the coarse grid, special prolongation operators have to be employed which are constructed from $A_{l}$ and the Galerkin coarse grid operator (3.86), see Hackbusch (1985). Wagner et al. (I997) use a coarse grid operator based on the Schur complement.

ANISOTROPIC PROBLEMS arise from an anisotropic diffusion tensor in

$$
-\nabla \cdot(K \nabla u)=f \quad \text { with } K=\left(\begin{array}{ll}
1 & 0 \\
0 & \epsilon
\end{array}\right) .
$$




\section{Finite Volume Discretization and Multigrid Solution}

Note that this equation changes it's elliptic character when $\varepsilon=0$ and is then of parabolic type. For $\epsilon \ll 1$ or $\epsilon \gg 1$, the convergence of multigrid with Jacobi- or Gauß-Seidel smoothers deteriorates quickly. One possible solution approach is called semi-coarsening, where the mesh is only refined in the direction of the strong coupling. This approach is attractive because it is possible for grids in two and three space dimensions, although the implementation requires a considerable effort for hybrid grids.

Another approach works by constructing robust smoothers for the problem. Block-line smoothers or modified ILU smoothers with an appropriate ordering of the unknowns have been proven to be very efficient (Wittum, I989). They are, however, not well suited for three-dimensional problems, as the approach requires the solution of two-dimensional subproblems in the smoother.

CONVECTION-DIFFUSION problems with dominating convection pose the most difficult challenge to multigrid convergence theory and practice. They can be studied from the model problem

$$
-\nabla \cdot(\mathrm{ru}-\epsilon \nabla \mathrm{u})=\mathrm{f} .
$$

with a flow field $\mathbf{r}$. A very effective solution strategy is available for the case of pure convection $(\epsilon=0)$ and flow fields without recirculation. In this case the stiffness matrix $A$ has lower triangular form if the unknowns are ordered in a downwind direction and an appropriate upwind scheme is chosen. The approach has been extended to flow fields with recirculation zones by several authors (Hackbusch, 1997, Bey and Wittum, 1997, Bey, 1998, Rentz-Reichert, 1996). It is also possible to approach the problem by constructing improved coarse grid correction matrices; see Reusken (2002) and the citations therein.

An increasingly popular class of multigrid methods are the algebraic multigrid methods, which do not rely on a given hierarchy of refined grids, but instead construct the grid hierarchy themselves from the given fine grid stiffness matrix. We did not pursue this research direction in this work, but we applied algebraic multigrid to some of the presented problems with good success. The work on algebraic multigrid methods was initiated by Ruge and Stüben (1987); of the large number of subsequent publications we mention Vaněk et al. (I996), Raw (I996), Braess (I995), Bank and Wagner (1999). To distinguish algebraic multigrid from the geometry-based multigrid algorithms, the latter is sometimes referred to as geometric multigrid.

An application of multigrid to a problem which bears some resemblance with our setting is the treatment of crack singularities in Brenner and Sung (I997). This can be considered the case of a lower-dimensional modeling of blocking fractures.

\subsubsection{Parallel and Adaptive Multigrid}

The numerical solution of linear systems of equations is accessible to acceleration by adaptivity and parallelization. Both approaches are employed for the presented discretization scheme to speed up the solution process. We do not comment extensively on this topic, because it 

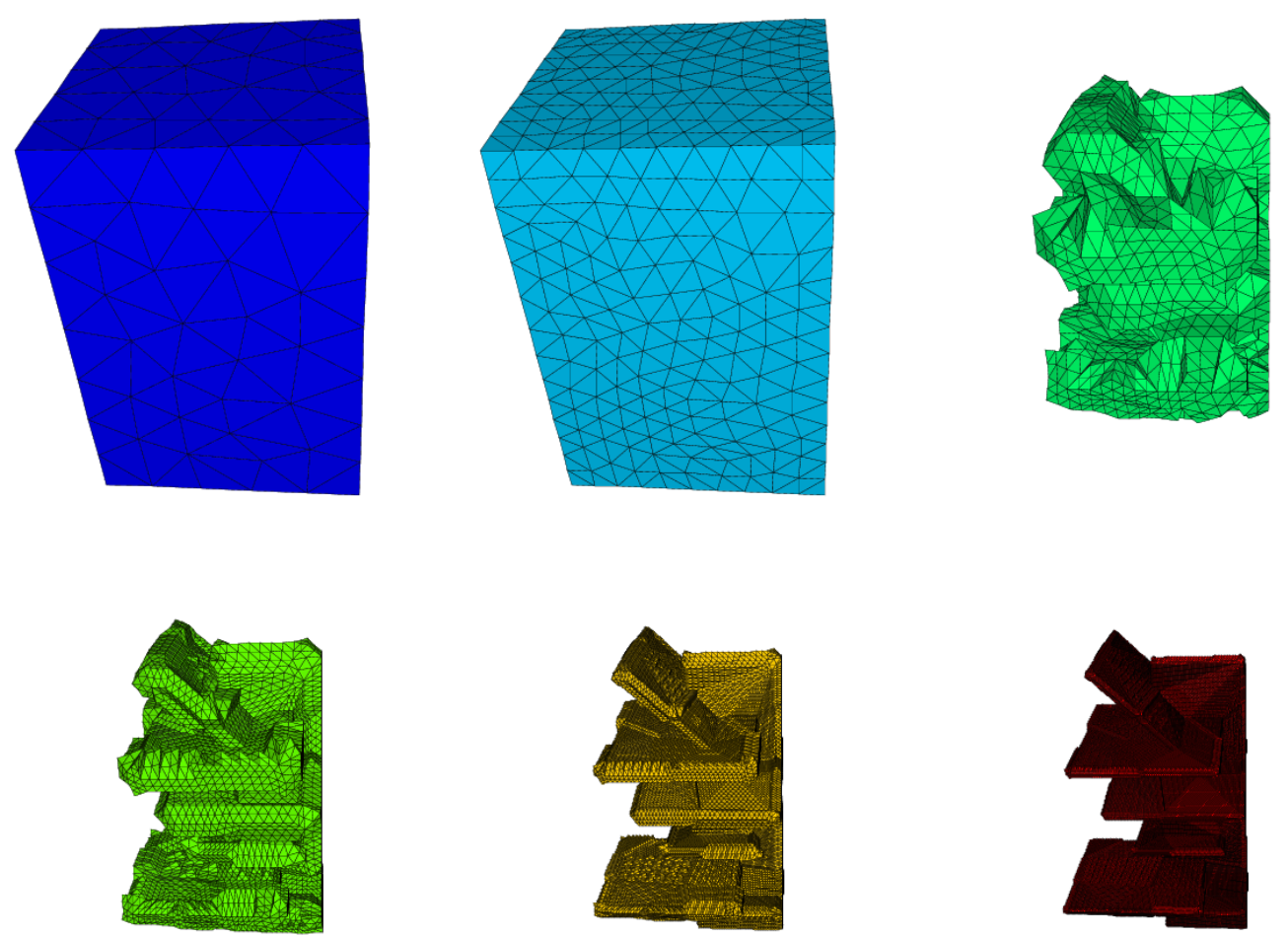

Figure 3.7.: Example of adaptive grid refinement of a fractured domain.

can be implemented in a problem-independent way and has received careful attention in Bastian (1996, 1999), Lang (2000)

It is possible to refine the initial coarse mesh either by error indicators (Verfürth, I996) or by prescribed refinement in specified regions. Two-phase flow problems are very well suited for adaptive refinement due to the local nature of fronts travelling through a medium, but we did not consider error estimation in this work. In figure 3.7 an adaptive refinement of a domain with eight fractures is shown on six grid levels. Starting at level 1 only the elements which share a vertex with a fracture are refined. As the refinement proceeds, the geometry of the fractures becomes visible. The refinement algorithm is rather complex, especially when combined with a parallel implementation. It is implemented in the toolbox UG as described in Lang (2000). The multigrid algorithm needs to be adjusted to the locally refined hierarchy. An analysis of the specific local multigrid method used here is given in Neuss and Wieners (2003); see also Bastian (I996).

The parallelization of the multigrid method is described in Bastian (I999). The implementation is done for MIMD parallel computers, because this is the only architecture offering scalability up to thousands of processors. Starting with a coarse grid stored on one processor, on each grid level a balanced mapping of elements to processors needs to be calculated. 


\section{Finite Volume Discretization and Multigrid Solution}

This is the load balancing problem. The distribution phase or load migration phase distributes elements to the processors. In the case of unstructured grids this involves the identification and consistent storage of dependencies among the geometrical objects nodes, edges, faces, and elements. The interaction of the components involved in the parallelization is described in Lang 2000)

\subsubsection{Multigrid for Systems}

The standard multigrid smoothers are not directly applicable to the Jacobian system, because some rows in the $A_{w w}$ block of the matrix may vanish. This problem of point-wise smoothers can be circumvented by using a point-block ordering and block variants of the smoothers. This approach was used in Bastian (I999), Bastian and Helmig (I999). We order the unknowns belonging to one vertex together, resulting in an ordering

$$
\tilde{\boldsymbol{z}}=\left(\mathbf{p}_{w, 1}, \mathbf{S}_{\mathfrak{n}, 1}, \ldots, \mathbf{p}_{w, N}, \mathbf{S}_{\mathfrak{n}, \mathrm{N}}\right)^{\top}
$$

which corresponds to a permutation of $z$ with the permutation matrix $Q$,

$$
\tilde{z}=\mathrm{Q} z
$$

Q transforms the system into the equivalent system

$$
\tilde{A} \tilde{z}=\tilde{\mathbf{b}}
$$

with

$$
\tilde{\mathrm{A}}=\mathrm{QAQ}^{\top}, \quad \tilde{\boldsymbol{z}}=\mathrm{Q} z, \quad \tilde{\mathbf{b}}=\mathrm{Q} \mathbf{b} .
$$

The matrix $A$ has a block structure with $N \times N$ blocks $\tilde{A}_{i j}$ of size $2 \times 2$,

$$
\tilde{A}=\left(\begin{array}{ccc}
\tilde{A}_{11} & \cdots & \tilde{A}_{1 N} \\
\vdots & & \vdots \\
\tilde{A}_{N 1} & \cdots & \tilde{A}_{N N}
\end{array}\right) .
$$

We may now ask under what conditions block variants of Jacobi, Gauß-Seidel and ILU iterations (which operate on the $2 \times 2$ blocks) can be applied. The only situation in which a block $\tilde{A}_{i j}$ can become singular is at boundaries where

$$
\rho_{w} \mathbf{u}_{w} \cdot \mathbf{n}=\phi_{w}, \quad S_{n}(\boldsymbol{x}, \mathbf{t})=1 .
$$

This boundary condition is not physically meaningful, since no water phase exists at points where $S_{n}=1$ holds, and consequently no condition can be imposed on the water flux.

The properties of the blocked versions of the smoothers are similar to the point-wise variants (Hackbusch, I994), but convergence of the Jacobi and the Gauß-Seidel iteration have not been proven for the considered problems. Convergence results are also missing for the ILU iteration, where even the existence of the ILU decomposition remains to be proven. 


\subsection{Computer Program Implementation}

\subsubsection{TRUNCATED RESTRICTION FOR DISCONTINUOUS PROBLEMS}

Bastian (I999) considers the case of a discontinuous diffusion coefficient which is 1 in one part of the domain and $\epsilon \ll 1$ in the other. Application of the standard grid transfer operators in the multigrid algorithm results in divergence if the jump in the discontinuity is large enough, because for nodes near the interface a correction on the order of $\mathcal{O}(1 / \epsilon)$ will be computed. In the truncated restriction, the restriction operator $R_{l}$ is replaced by

$$
\left(\tilde{R}_{l}\right)_{i j}=\left(R_{l}\right)_{i j} \cdot \min \left(c, \frac{\left(A_{l}\right)_{i j}}{\left(A_{l-1}\right)_{i j}}\right) \text {, }
$$

with a constant $c$. Note that the modified restriction operator $\left(\tilde{R}_{l}\right)_{i j}=\left(R_{l}\right)_{i j}\left(A_{l}\right)_{i j} /\left(A_{l-1}\right)_{i j}$ results from a scaling of the equation $A x=b$ with $D=\operatorname{diag}(A)$. The additional choice of the minimum prevents exactly the problems at interior discontinuous boundaries. By choosing $c \geq 1$ the standard restriction operator results for constant coefficients, since in this case $\left(A_{l}\right)_{i j} /\left(A_{l-1}\right)_{i j} \leq 1$ holds at all interior vertices. At restrictions of interior vertices to Neumann boundary vertices, $\left(A_{l}\right)_{i j} /\left(A_{l-1}\right)_{i j}$ may be larger than 1 , so we choose $c=2$ in all subsequent computations. Numerical experiments indicate that the choice of $c$ is not crucial as long as it is smaller than 5 . This method is called the diagonally scaled/truncated restriction multigrid algorithm, DSTR-MG. Bastian (1999) contains examples for which the standard multigrid iteration diverges, but the modified multigrid iteration with truncated restriction shows grid independent convergence or $\mathcal{O}(\mathrm{J})$ behavior (with the number of grid levels $\mathrm{J}$ ).

The extension to systems is done for the point-block ordered system. Instead of scaling with the diagonal matrix, the system is scaled with the point-block diagonal matrix $\hat{D}$, and the resulting restriction is

$$
\hat{\mathrm{R}}_{l}=\hat{\mathrm{D}}_{l-1}^{-1} \mathrm{R}_{l} \hat{\mathrm{D}}_{l}
$$

The $2 \times 2$ blocks which constitute $\hat{R}_{l}$ are determined by

$$
\left(\hat{R}_{l}\right)_{i j}=\left(R_{l}\right)_{i j}\left(\hat{D}_{l-1}^{-1}\right)_{i j}\left(\hat{D}_{l}\right)_{i j} .
$$

The truncated restriction for systems is defined as

$$
\left.\left(\left(\hat{R}_{l}\right)_{i j}\right)_{\alpha \beta}=\left(R_{l}\right)_{i j} \cdot \max \left(0, \min \left(c,\left(\left(\hat{D}_{l-1}\right)_{i j}^{-1}\left(\hat{D}_{l}\right)_{i j}\right)\right)_{\alpha \beta}\right)\right) .
$$

\subsection{Computer Program Implementation}

\subsection{The SIMULATION ENVIRONMENT}

The numerical simulation of multi-phase flow in fractured porous media requires several software components which need to interact as depicted in figure 3.8. The components in figure 3.8 are employed in many different application fields and we want to comment on the special requirements of the present application.

The geometry of domains in the subsurface can be very complex and can in all but the simplest cases only be resolved by unstructured grids. The occurrence of sharp front suggests 


\section{Finite Volume Discretization and Multigrid Solution}

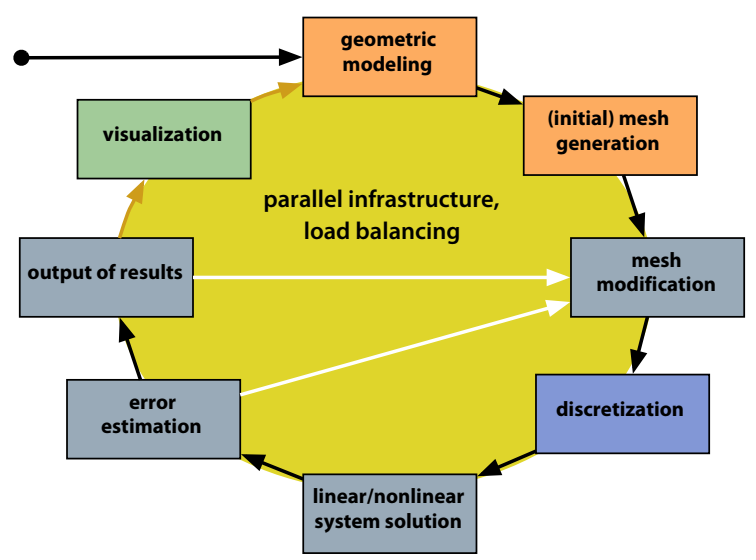

Figure 3.8.: The components of a numerical simulation environment and their interaction.

that adaptive grid refinement is employed near the front. A combination of both features is complicated to implement, but for detailed simulations it is even necessary to run simulations on parallel computers with distributed memory. The combination of unstructured grids, adaptivity and parallelization introduces complexity into the code development which is by orders of magnitude greater than for structured, uniformly refined grids on a single processor computer. Since it is not reasonable to implement this functionality individually for each application domain, the framework UG (Bastian et al., 1997) was developed, which provides the mentioned functionality in a problem-independent way. The code developed for the solution of the two-phase equations is part of a larger simulation environment, which contains different models for subsurface flow and transport.

In this chapter we describe some core features of the framework UG and explain how the implementation of the module for fractured porous media is done based on this framework. We also explain some crucial components of the discretization process, namely the generation of fractured domains in a pre-processing step and the visualization of fractured domains in post-processing.

\subsubsection{THE NUMERICAL FRAMEWORK UG}

UG (Bastian et al., I997) was written to provide a framework on which state-of-the-art simulation environments can be built. Many components that are required for the finite element or finite volume simulation of processes described by partial differential equations are independent of the problem, but are so complex that they cannot be implemented by one developer alone. With a framework like UG, developers can focus on modeling, discretization or solvers and don't need to know how load balancing, parallel load migration work in detail.

Domain Module The domain module can represent two-dimensional and three-dimensional geometries. With the domain manager module domain boundaries can be defined by means of boundary patches and domains can be split into several subdomains (with 
different material properties). It also handles the treatment of boundary conditions, so that for given nodes or element sides of the grid the user program can determine which boundary condition is valid in a given location. This works also if the grid is distributed over several processors. Inner boundaries are used to describe fractures and to associate a virtual width with each point on the fractures.

Grid Manager UG can handle triangles and quadrilaterals for two-dimensional geometries and tetrahedrons, pyramids, prisms and hexahedrons for three-dimensional geometries. This variety of element types is necessary to maintain consistent grids in adaptive refinement (i. e. no hanging nodes will occur). The different element types also offer flexibility in the triangulation of complicated geometries.

Local grid refinement greatly reduces storage requirements for problems where sharp fronts or singularities in the solution require grid refinement only in certain regions of the domain.

Grids are stored in a hierarchical fashion. The hierarchical viewpoint is maintained throughout all components of the UG framework and is used to ensure scalability of all components.

Automatic Grid Generation Interfaces to different grid generator softwares exist as well as two grid generators which are included with UG, one for two-dimensional domains and one for three-dimensional domains. Additionally there are interfaces to several other grid generators.

User Data Manager The basic vector-matrix data structure is very flexible and allows for the attachment of degrees of freedom with nodes, edges, faces or elements. Based on the user data managers functionality, finite element methods and finite volume methods can be implemented, from simple node based schemes to complex higherorder methods.

Numerical Algorithms The numerical algorithms for the solution of linear and non-linear systems as well as the time-stepping schemes are organized in a class hierarchy. The object-oriented approach makes designs of solutions schemes possible which are structurally clear, easily configurable and extensible. The algorithms are implemented in a problem-independent way. Components of a solution scheme can be chosen from a wide range of implemented classes.

Script Language UG applications are driven by a script language. Its syntax is similar to C. UG applications can either be run in batch mode by executing scripts, or interactively.

Visualization module The visualization module of UG was designed in a scalable way, so that large parallel simulations can be visualized in an efficient way. It employs the hierarchical data structure and is parallelized, thus avoiding unnecessary calculations in the process. Output can be drawn to the screen or to PostScript or PPM files (as well as to a native picture format).

For more sophisticated visualization it is possible to write data in several visualization program formats: OpenDX/DataExplorer, TecPlot, GRAPE and AVs 


\section{Finite Volume Discretization and Multigrid Solution}

$I / O$ and restart In long simulation runs it is often necessary to save intermediate results from which the calculation can be restarted if a hardware error occurs and prevents the simulation from finishing. On parallel computers with several hundreds of processors, this event is much more common than scientists would hope, and on many large computers there is a time limit for individual jobs which is easily exceeded by large simulation runs. In both cases the restart functionality is necessary.

Message Passing Parallelization UG is parallelized by a domain decomposition approach. An underlying framework, DDD (Dynamic Distributed Data) is responsible for the consistency of the data structures during all stages of the lifetime of an application, especially after modification and distribution of the grid. DDD is also responsible for packing messages, sending them to processors and unpacking them. The passing of messages is done with the functionality of the underlying Parallel Processor Interface (PPIF), which uses MPI, PVM or vendor-dependent message passing mechanisms.

Software Engineering The large complexity of UG results in a code basis of over 350.000 lines which were written in more than twelve years by seven main developers and numerous other contributors.

All these components work regardless of the underlying physical problem. If solver components are not suitable for the underlying problem it is usually easy to extend the concerning module by inheriting from the solver class and then modifying or extending its functionality.

Knowledge about the physical problem is part of the problem classes. These modules are implemented on top of UG and contain one or several discretizations of the mathematical description of the physical problem along with problem specific functionality (like e.g. constitutive relationships.

\subsubsection{PRE- AND POST-PROCESSING}

Although the simulation environment contains all components mentioned in figure 3.8 , the components geometric modeling, mesh generation, and visualization are more loosely coupled to the simulation environment than the others. It is easier to use different visualization programs or grid generators since they can normally be connected by a file interchange format.

\section{DOMAIN GENERATION}

In fractured systems the exact location of the fractures is often not known, but a realistic approximation of the fracture network is crucial for the simulation process. In these situations a fracture generator can be employed to generate fracture networks based on prescribed geological data. The program $\mathrm{FRAC}_{3} \mathrm{D}$ (Silberhorn-Hemminger, 2002), developed by A. Hemminger at the IWS, Stuttgart, can create a discrete structural model of a fractured domain based on data collected in the laboratory or the field. Domains created by FRAC $3 \mathrm{D}$ can be converted to a format readable by UG. 


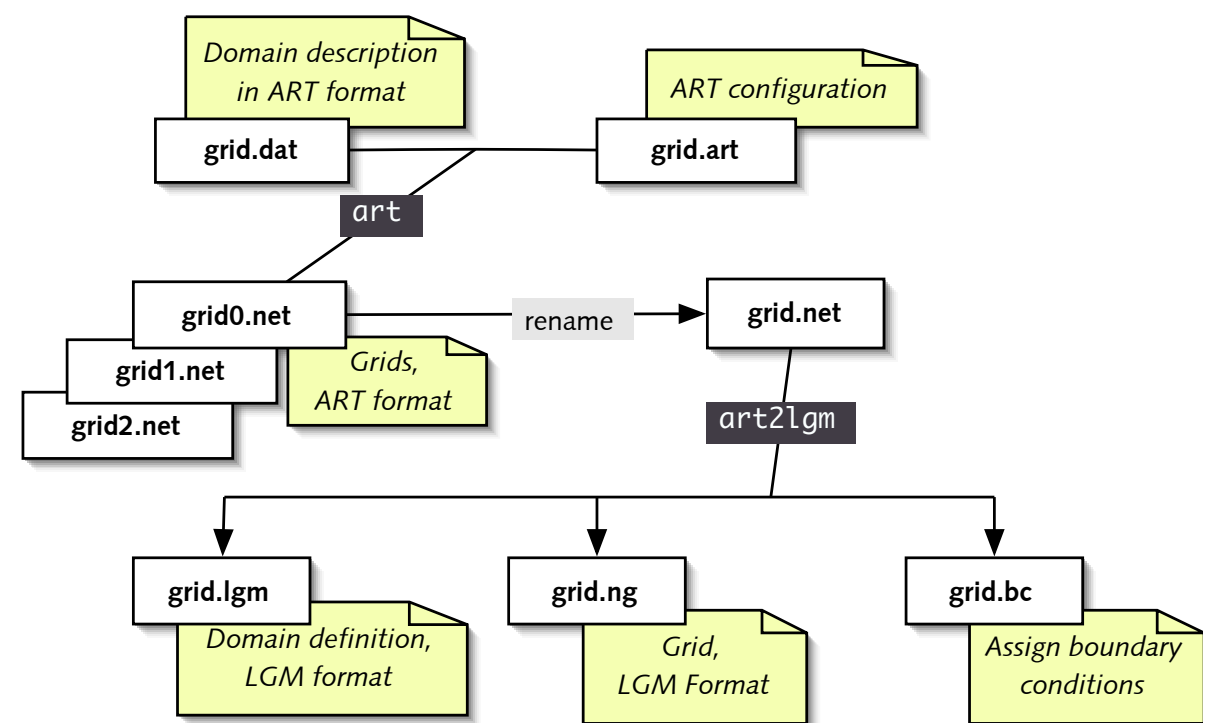

Figure 3.9.: The grid generator ART creates grids from domains descriptions generated with the fractured domain generator $\mathrm{FRAC}_{3} \mathrm{D}$. The resulting grid file is used for conversion to the LGM-Domain format. Domain as well as grid representation are taken from the grid file.

\section{Mesh generation}

The next step in the simulation process is the creation of an initial mesh. For the geometric multigrid method it is advantageous to have a coarse initial mesh, but without degenerate elements (i. e. elements with large aspect ratios or large inner angles). The amount of work necessary to create these meshes by hand is prohibitive, so automatic grid generation has to be employed. Fuchs (I999) developed the grid generator ART3D in close collaboration with the research groups in Heidelberg and Stuttgart to meet the special demands of these problems. The lower-dimensional modeling of the fractures is advantageous in the grid generation process, because fractures have only to be treated like inner boundaries. This is much easier than the mesh generation for fracture-matrix systems which are represented as thin layers. In the latter case, adaptive grid generation has to be employed to avoid the creation of excessively many elements in the surrounding rock matrix. An interesting concept was introduced by Kornhuber (e. g. Neunhäuserer et al. (2002)), who proposed to employ mesh generation for the domain with fractures represented as inner boundaries, and in a post-processing step to extend the fractures to the full dimensionality. This has to be done carefully in order not to deteriorate the element quality in fracture intersections. The numerical results presented later suggest that the different possible shapes of the fracture ends (triangles or quadrilaterals in two space dimensions) obtained by this procedure have a significant influence on fluid flow and transport.

Figure 3.9 explains the process of how a mesh is created from the domain description file and how domain description and mesh are converted to LGM format (a UG-native domain and grid format). The converter art2lgm is a PERL-script, which reads the grid file created 


\section{Finite Volume Discretization and Multigrid Solution}
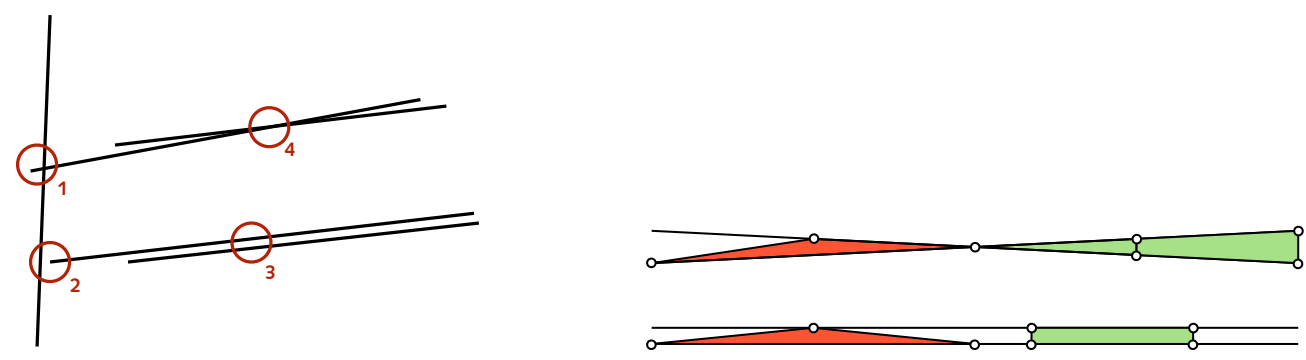

Figure 3.IO.: Four domain features which are difficult for automatic mesh generation (left) and possible elements of good quality (green) and poor quality (red) for parallel and almost parallel fractures (right).

by ART3D and produces an LGM-domain description file and an LGM-grid description file. Only the ART3D-grid file is used for conversion, because this circumvents some complex geometrical calculations which would otherwise be necessary to determine the orientation of the domain boundaries. art2lgm uses all element sides on the domain boundary to create a triangulated boundary description. These boundary sides are used as boundary patches in UG. The third file that is created associates the boundary patches with the appropriate boundary conditions.

The automatic generation of meshes has persisted as one of the most challenging tasks of the simulation process. The mesh generation for fractured porous media has to treat four situations which are especially difficult. They are depicted in figure 3.IO for a twodimensional domain, but the even more severe difficulties for three-dimensional domains can be traced back to these fundamental situations:

I. Fracture intersections, where one fracture end protrudes only slightly from the intersection. A fine mesh is required in the vicinity of this region, which should coarsen rapidly outside this region. In three-dimensional domains, the protruding fracture end can have a "difficult" shape, e. g. a very flat triangle.

2. Almost meeting fractures, where one fracture ends in close proximity to the other. The mesh should also be of high resolution only near this region.

3. Parallel fractures very close to each other should be meshed with quadrilaterals in two space dimensions and with hexahedrons or prisms in three space dimensions, because they can prevent the generation of elements with large inner angles.

4. Fractures intersecting at a very small angle can lead to the generation of elements with very large angles.

In both latter cases, automatic mesh generation of Delaunay-type is especially susceptible, because the mesh generation algorithm minimalizes a penalty functional which places vertices as far apart from each other as possible. In the case of parallel or almost parallel fractures this leads necessarily to large angles, where two vertices on the two fractures, placed close to each other, would produce preferable elements. This is illustrated on the right in figure $3 . \mathrm{IO}$. 


\subsection{Computer Program Implementation}

Even if the green quadrilateral elements are divided into triangles, their largest angles are still close to $90^{\circ}$, while the red elements largest angles are close to $180^{\circ}$.

\section{VISUALIZATION}

The visualization of fractured porous media problems requires the representation of lowerdimensional elements. We implemented an interface to the visualization software OpenDX, in which lower-dimensional elements can be realized and which can be combined in the visualization with volumetric elements. We also realized an interface to the visualization environment COVISE, where the virtual reality capabilities have been very helpful in discovering features of the solution which are otherwise difficult to detect. 
3. Finite Volume Discretization and Multigrid Solution 


\section{The Discontinuous Galerkin Method for ELLIPTIC PROBLEMS}

The discontinuous Galerkin method (DG) is an attractive discretization scheme for fluid flow problems in porous media, because it is locally mass conservative like the finite volume method but also allows for higher order approximation and has relaxed requirements on the mesh. There is a large number of discretization methods which are all titled discontinuous Galerkin methods and they have been applied to such diverse problems as groundwater flow and multiphase flow (on which we will comment below), Euler and Navier-Stokes equations (Oden and Baumann, 2000, Bassi and Rebay, I997b, 2000), semiconductor device simulation (Chen et al., I995a b), Maxwell's equations (Warburton and Karniadakis, I999, Warburton, 2000), Hamilton-Jacobi equation ( $\mathrm{Hu}$ and Shu, 2000) and neutron transport (Reed and Hill, 1973). We apply one discontinuous Galerkin method to the groundwater flow equation and compare its performance for fractured domains with the finite volume method of the previous chapter. The element type in the fractures are volumetric elements, not lower-dimensional elements as for the multiphase flow discretization.

The next section contains a short overview of (some) discontinuous Galerkin methods. Then we present the bilinear form employed for our discretization, explain the fast multigrid solution of the systems and present numerical results.

\section{I. Development of the Discontinuous Galerkin Method}

Discontinuous Galerkin methods derive their name from the choice of trial and test functions which are discontinuous across element boundaries and continuous within elements. The development of the discontinuous Galerkin method is reviewed in Cockburn et al. (2000a); an overview of the state of the art in 2000 is the collection Cockburn et al. (2000c).

The original discontinuous Galerkin method was presented by Reed and Hill (1973) for the solution of the neutron transport equation, a stationary linear hyperbolic problem of the form

$$
\nabla \cdot\{\boldsymbol{a} u\}+\sigma u=f \quad \text { in } \Omega .
$$

$\sigma$ is a real number and $\boldsymbol{a}$ is a constant vector. If we multiply the equation by a test function $v$ and integrate over the element domain $\Omega_{e}$ of an element $e$ from a triangulation $E_{h}$ of $\Omega$, we get after integration by parts

$$
-\int_{\Omega_{e}} u \boldsymbol{a} \cdot \nabla v \mathrm{~d} x+\int_{\partial \Omega_{e}} \boldsymbol{a} \cdot \mathbf{n u v} \mathrm{d} s+\sigma \int_{\Omega_{e}} u v d x=\int_{\Omega_{e}} f v d x .
$$


$\boldsymbol{n}$ is the outward unit normal to element $e$. To get an approximation $u_{h}$ of $u$, we choose the test functions to be polynomials of degree at most $k$ on the elements of the triangulation. The space of polynomials of degree at most $k$ on element $e$ is denoted by $P_{k}\left(\Omega_{e}\right)$. Now we determine $u_{h}$ as the solution of: Find $u_{h}$ such that for all $e \in E_{h}$ and all $v \in V \in P_{k}\left(\Omega_{e}\right)$

$$
-\int_{\Omega_{e}} u_{h} \boldsymbol{a} \cdot \nabla v d x+\int_{\partial \Omega_{e}} \boldsymbol{a} \cdot \boldsymbol{n}_{e} \hat{u}_{h} v d s+\sigma \int_{\Omega_{e}} u_{h} v d x=\int_{\Omega_{e}} f v d x
$$

with the numerical flux

$$
\hat{u}_{h}(x)=\lim _{s \rightarrow 0+} u_{h}(x-s a) .
$$

The value $\hat{u}_{h}(x)$ is the upwind value of $u_{h}$ upstream of the characteristic direction $a$. When the elements are ordered according to the characteristic direction of $a$, the $u_{h}$ values can be computed element by element from the upstream values of $u_{h}$ hitting $\partial \Omega_{e}$.

The discontinuous Galerkin can be employed as a time differencing scheme as well, see Thomée (1997) and Eriksson et al. (1996). The first analysis of the discontinuous Galerkin method as a time differencing scheme was done in Lesaint and Raviart (1974) and Jamet (1978), Eriksson et al. (I985) studied the method for parabolic equations. In the context of ordinary differential equations the first analysis was done by Delfour et al. (I98I). A posteriori error control was pioneered by Johnson et al. (I990).

A series of papers by Cockburn and Shu (Cockburn and Shu, I991, I989, Cockburn et al., I989, I990, Cockburn and Shu, I998b), where the discontinuous Galerkin method was employed for nonlinear time-dependent hyperbolic problems, propelled the interest in the method. They investigated the discontinuous Galerkin discretization in space with the explicit TVD Runge-Kutta time discretization by Shu (1988), together with limiter methods in the multidimensional case to ensure nonlinear stability and convergence to the entropy solution.

In I998, Cockburn and Shu (I998a) introduced the local discontinuous Galerkin method (LDG), based on the work of Bassi and Rebay (1997a) for the compressible Navier-Stokes equations, and proved stability and error estimates for this method which is suited for convection-dominated convection-diffusion problems. The LDG method is based on rewriting the equation as a first order system and then discretizing by the DG method. Nonlinear stability without flux limiters can be achieved for this method by a careful choice of the rewriting and the numerical fluxes. The LDG method is locally mass conservative, which is a property that in general is difficult to achieve for higher-order finite element methods. If LDG is applied to elliptic equations the method can be ill-posed for some choices of the numerical fluxes (e.g. the choice by Bassi and Rebay in their original DG scheme).

A discontinuous Galerkin method suitable for elliptic problems was introduced in Baumann (1997), Oden et al. (I998), Baumann and Oden (I999). This is the method we are going to employ below and we would like to explain the evolution of this method. The ingredient with the oldest roots is the incorporation of Dirichlet boundary conditions in a weak form through penalty terms in the standard finite element method (Nitsche, I97I, Lions, 1968). In order to solve

$$
-\Delta u=f \text { in } \Omega, \quad u=g \text { on } \partial \Omega
$$




\section{I. Development of the Discontinuous Galerkin Method}

one uses the weak form

$$
\int_{\Omega} \nabla \mathrm{u} \cdot \nabla v \mathrm{~d} x+\int_{\partial \Omega} \mu(\mathrm{u}-\mathrm{g}) v \mathrm{~d} s=\int_{\Omega} \mathrm{f} v \mathrm{~d} x \quad \forall v \in \mathrm{H}^{1}(\Omega) .
$$

The traditional way to impose Dirichlet boundary conditions is by an appropriate choice of the trial functions. Note that the trial functions above are not zero at the Dirichlet boundary. The penalty parameter $\mu$ is mesh-dependent.

In this setting the trial and test functions are still continuous. The interior penalty Galerkin finite element method (IP) (Wheeler, I978, Percell and Wheeler, 1978) uses discontinuous basis and trial functions and ensures continuity of the solution at inter-element boundaries by penalties. The method was extended to nonlinear elliptic and parabolic equations in Arnold (1979, 1982). The IP method is symmetric and locally mass conservative, and optimal error estimates could be proven, but it hasn't found much practical application because the resulting systems are indefinite and very difficult to solve, and because there is no way to find an optimal choice of the penalty parameter $\mu$ for particular problems; it has to be chosen large enough to make the bilinear form coercive.

In his thesis, Baumann (I997) proposed a new stabilization. It is non-symmetric and does not require a penalty term. It was analyzed in Oden et al. (1998) and Rivière (2000), Rivière et al. (1999), Rivière and Wheeler (2000), where also the non-symmetric interior penalty method (NIPG) was introduced which contains an additional penalty term. The relations between LDG, Baumann-Oden DG, IP and NIPG are explored in Arnold et al. (2002); their performance is assessed in Castillo (2002). Applications to porous medium problems can be found in Rivière (2000), Rivière et al. (2000), Aizinger et al. (200I). The DG method presented here has been extended to transport in Bastian and Lang (2002) and two-phase flow in an IMPES formulation in Bastian (2002).

The discontinuous Galerkin methods developed in recent years have several advantages which make them competitive or even superior to standard finite element methods.

$\triangleright$ Given that the problem possesses enough regularity, the methods are higher order convergent. For many methods, the convergence rates are optimal in the $\mathrm{L}_{2}$ and $\mathrm{H}^{1}$ norm.

$\triangleright$ The methods can be applied to elliptic, parabolic and hyperbolic problems.

$\triangleright$ The methods are locally mass conservative.

$\triangleright$ For elliptic problems fast multigrid solvers are available (Bastian and Reichenberger, 2000, Gopalakrishnan and Kanschat, 2003, Hemker and van Raalte, 2002).

$\triangleright$ The methods can be employed on unstructured, non-matching grids, which makes them very flexible and easy to implement. This makes $h$-adaptivity particularly easy. Additionally, they provide an easy access to $\mathrm{hp}$-adaptive methods, given the availability of a p-estimator.

$\triangleright$ For time-dependent problems suitable high-order explicit as well as implicit time discretization are available. 
$\triangleright$ Modern, cache-based computer architectures are utilized efficiently since matrices with small dense blocks arise and the method is not limited as much by memory throughput as is the case for low-order unstructured finite element and finite volume methods.

\subsection{Formulation OF THE BILINEAR FORM}

We consider the groundwater equation (2.8) with constant density and viscosity,

$$
\begin{aligned}
-\nabla \cdot\left(\rho \frac{\mathrm{K}}{\mu} \nabla p\right) & =\rho \mathbf{q} & & \text { in } \Omega \subset \mathbb{R}^{\mathrm{d}}, \mathrm{d}=2,3 . \\
p & =p_{\mathrm{d}} & & \text { on } \Gamma_{\mathrm{d}} \\
\mathbf{u} \cdot \mathfrak{n} & =\phi & & \text { on } \Gamma_{\mathfrak{n}} \quad \text { with } \quad \mathbf{u}=-\rho \frac{\mathrm{K}}{\mu} \nabla p
\end{aligned}
$$

Let $\mathrm{E}_{\mathrm{h}}$ denote a subdivision of $\Omega$, which does not have to be a triangulation

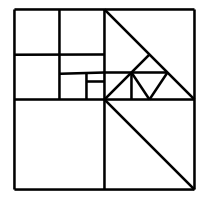

Admissible mesh (i. e. hanging nodes are allowed) and which may contain elements of mixed type. An example for an admissible mesh is shown here. For the basis and test functions we choose the space of polynomial functions of degree $k$ on element $e \in \mathrm{E}_{\mathrm{h}}$

$$
P_{k}\left(\Omega_{e}\right)=\left\{p: \Omega_{e} \rightarrow \mathbb{R} \mid p(x, y)=\sum_{0 \leq a+b \leq k} c_{a b} x^{a} y^{b}\right\} .
$$

$\mathrm{P}_{\mathrm{k}}$ can be used as the basis for triangles and quadrilaterals; alternatively we can choose the basis functions from the polynomial space

$$
\mathrm{Q}_{k}\left(\Omega_{e}\right)=\left\{p: \Omega_{e} \rightarrow \mathbb{R} \mid p(x, y)=\sum_{0 \leq a, b \leq k} c_{a b} x^{a} y^{b}\right\}
$$

which is the more natural choice for quadrilaterals. The extension to three space dimensions is obvious.

In the implementation of our computer program the bases of $P_{k}$ and $Q_{k}$ are chosen as $\mathrm{L}_{2}$-orthonormal polynomials, which are generated from the monomials by Gram-Schmidt orthonormalization. The shape of the resulting functions is depicted in figure 4.I. The dimension of the space spanned by the basis functions for a choice of the degree $k-i$. e. the number of unknowns per element-is shown in table 4.I.

We choose the space of trial functions to be

$$
V^{k}\left(E_{h}\right)=\prod_{e \in E_{h}} P_{k}\left(\Omega_{e}\right)
$$

The interior boundary between element $e$ and $f$ (edges in $2 \mathrm{D}$ and faces in $3 \mathrm{D}$ ) is denoted by

$$
\Gamma_{\text {ef }}=\partial \Omega_{e} \cap \partial \Omega_{\mathrm{f}}
$$




\begin{tabular}{lllllrrrrrr}
\hline & $\mathrm{k}=$ & 0 & $\mathrm{I}$ & 2 & 3 & 4 & 5 & 6 & 7 & 8 \\
\hline $\mathrm{d}=2$ & $\mathrm{P}_{k}$ & 1 & 3 & 6 & 10 & 15 & 21 & 28 & 36 & 45 \\
& $\mathrm{Q}_{k}$ & 1 & 4 & 9 & 16 & 25 & 36 & 49 & 64 & 81 \\
\hline $\mathrm{d}=3$ & $\mathrm{P}_{k}$ & 1 & 4 & 10 & 20 & 35 & 56 & 84 & 120 & 165 \\
& $\mathrm{Q}_{k}$ & 1 & 8 & 27 & 64 & 125 & 216 & 343 & 512 & 729 \\
\hline
\end{tabular}

Table 4.I.: Number of unknowns in one element for the DG method.

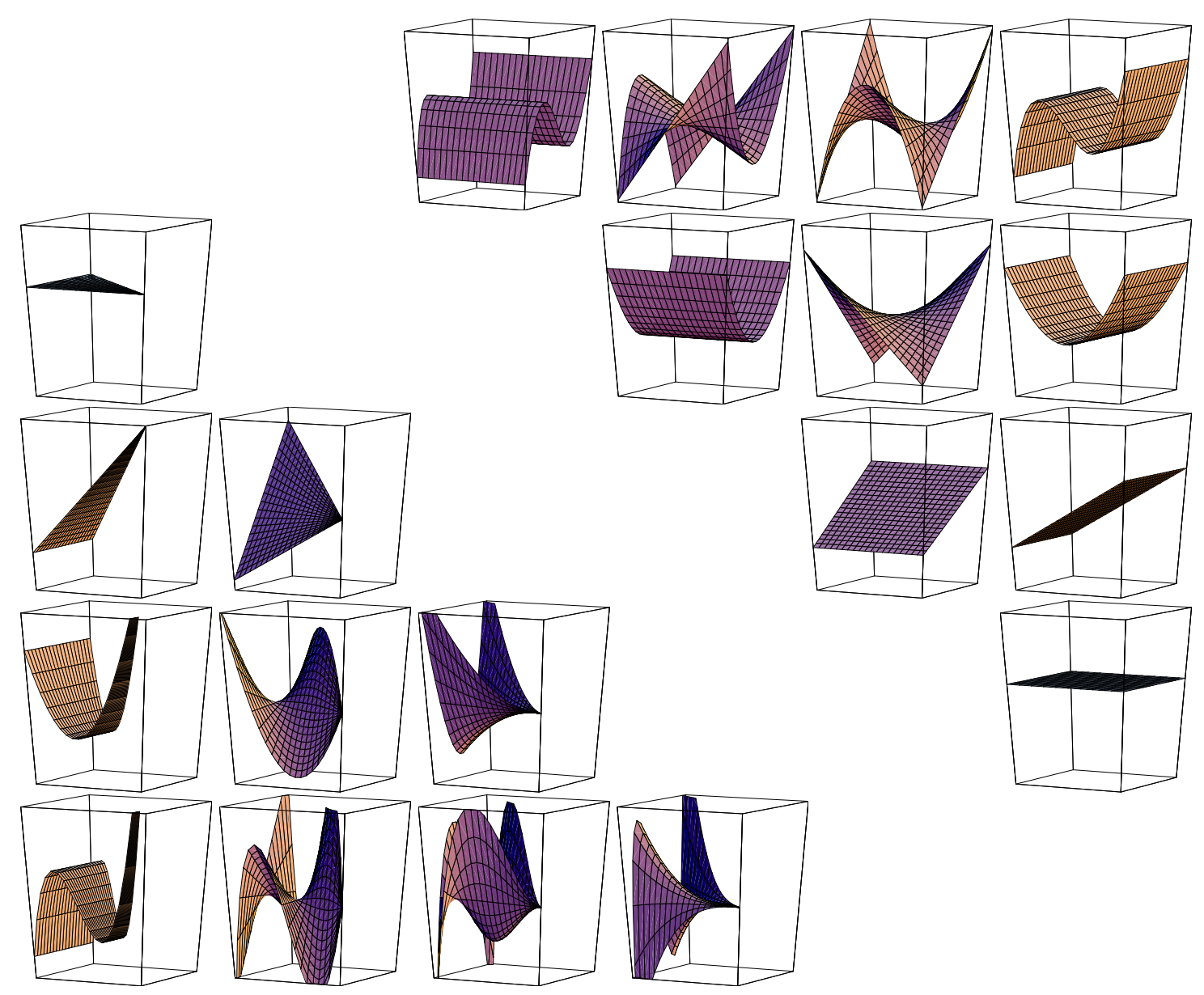

Figure 4.I.: Discontinuous Galerkin method basis functions. In the upper triangle are the $\mathrm{P}_{2}$ basis functions on the quadrilateral element, in the lower triangle the $\mathrm{P}_{2}$ basis functions for the triangular element. 


\section{The Discontinuous Galerkin Method for Elliptic problems}

The set of all interior edges is

$$
\Gamma_{\text {int }}=\bigcup_{e, f \in E_{h}, e \neq f} \Gamma_{e f} .
$$

With every interior edge $\Gamma_{\text {ef }}$ we associate a unique unit normal vector $\boldsymbol{n}$. A possible choice is to let $\boldsymbol{n}$ point from element $e$ to $f$ if $e>f$ and from $f$ to $e$ otherwise. For any point $x \in \Gamma_{e f}$ we define the jump of a function $v \in \mathrm{V}^{\mathrm{k}}\left(\mathrm{E}_{\mathrm{h}}\right)$ by

$$
[v](x)=\lim _{\epsilon \rightarrow 0+} v\left(x+\epsilon n_{e f}\right)-\lim _{\epsilon \rightarrow 0+} v\left(x-\epsilon n_{e f}\right)
$$

and the average by

$$
\langle v\rangle(x)=\frac{1}{2}\left(\lim _{\epsilon \rightarrow 0+} v(x+\epsilon \mathfrak{n})+\lim _{\epsilon \rightarrow 0+} v(x-\epsilon \mathfrak{n})\right) .
$$

The identity

$$
[v w]=[v]\langle w\rangle+\langle v\rangle[w]
$$

is easily verified.

Let us now go back to 4.7a and derive the bilinear form for the discretization. We consider one element $e \in \mathrm{E}_{h}$ and multiply with a test function $v \in \mathrm{P}_{k}\left(\Omega_{e}\right)$. After integration by parts we get

$$
\int_{\Omega_{e}} \rho \frac{\mathrm{K}}{\mu} \nabla p \cdot \nabla v \mathrm{~d} x-\int_{\partial \Omega_{e}} \rho \frac{\mathrm{K}}{\mu} \nabla p \cdot \mathfrak{n} v \mathrm{ds}=\int_{\Omega_{e}} \mathrm{q} v \mathrm{~d} \boldsymbol{x} \quad \forall v \in \mathrm{P}_{\mathrm{k}}\left(\Omega_{e}\right)
$$

Summation over all elements in $E_{h}$ and collecting the boundary integrals into interior and exterior edges results in

$$
\begin{aligned}
\text { (4.I6) }-\sum_{e \in \mathrm{E}_{\mathrm{h}}} \int_{\Omega_{e}} \rho \frac{\mathrm{K}}{\mu} \nabla p \cdot \nabla v \mathrm{~d} x & +\sum_{\Gamma_{\mathrm{ef}} \in \Gamma_{\text {int }} \int_{\Gamma_{e f}}\left[\rho \frac{K}{\mu} \nabla p \cdot \mathfrak{n} v\right] \mathrm{ds}} \\
& +\sum_{\Gamma_{e} \in \partial \Omega} \int_{\Gamma_{e}} \rho \frac{\mathrm{K}}{\mu} \nabla p \cdot \mathfrak{n} v \mathrm{ds}=\sum_{\mathrm{e} \in \mathrm{E}_{\mathrm{h}}} \int_{\Omega_{e}} \mathrm{q} v \mathrm{~d} x \quad \forall v \in \mathrm{V}^{\mathrm{k}}\left(\mathrm{E}_{\mathrm{h}}\right)
\end{aligned}
$$

The interface between two neighboring elements is handled by the second term on the left side. For second order elliptic problems there are two interface conditions: The first interface condition is continuity of the pressure, $[p]=0$. Second, the normal flux should be continuous, which can be expressed as

$$
\left[\rho \frac{K}{\mu} \nabla p \cdot \mathfrak{n}\right]=0
$$

and which is incorporated into the second term by

$$
\left[\rho \frac{K}{\mu} \nabla p \cdot \mathfrak{n} v\right]=\left[\rho \frac{K}{\mu} \nabla p \cdot \mathfrak{n}\right]\langle v\rangle+\left\langle\rho \frac{K}{\mu} \nabla p \cdot \mathfrak{n}\right\rangle[v]=\left\langle\rho \frac{K}{\mu} \nabla p \cdot \mathfrak{n}\right\rangle[v] .
$$


The bilinear form for the discontinuous Galerkin method follows from 4.16 by inserting the flux boundary conditions, splitting the boundary term into Dirichlet and Neumann parts and collecting terms independent of $p$ on the right hand side. The penalty on the jump of $p$ at interior edges is included in the second sum of $a_{h}$.

$$
\begin{aligned}
& \mathrm{a}_{\mathrm{h}}(\mathrm{p}, v)=\sum_{\mathrm{e} \in \mathrm{E}_{\mathrm{h}}} \int_{\Omega_{e}} \rho \frac{\mathrm{K}}{\mu} \nabla p \cdot \nabla v \mathrm{~d} x \\
& +\sum_{\Gamma_{e f} \in \Gamma_{\text {int }} \int_{\Gamma_{e f}}}\left\langle\rho \frac{K}{\mu} \nabla v \cdot \mathfrak{n}\right\rangle[p]-[v]\left\langle\rho \frac{K}{\mu} \nabla p \cdot \mathfrak{n}\right\rangle d s \\
& +\sum_{\Gamma_{e} \in \Gamma_{\mathrm{d}}} \int_{\Gamma_{e}}\left(\rho \frac{\mathrm{K}}{\mu} \nabla v \cdot \mathfrak{n}\right) p-v\left(\rho \frac{\mathrm{K}}{\mu} \nabla p \cdot \mathfrak{n}\right) \mathrm{ds} . \\
& l_{h}(v)=\sum_{e \in E_{h}} \int_{\Omega_{e}} q v d x+\sum_{\Gamma_{e} \in \Gamma_{d}} \int_{\Gamma_{e}}\left(\rho \frac{K}{\mu} \nabla v \cdot \mathfrak{n}\right) p_{d} d s+\sum_{\Gamma_{e} \in \Gamma_{n}} \int_{\Gamma_{e}} v \phi d s
\end{aligned}
$$

The Baumann-Oden discontinuous Galerkin method by Baumann and Oden (I999), Baumann (I997), Oden et al. (I998) is: Find $p \in V^{k}\left(E_{h}\right)$ such that for all $v \in \mathrm{V}^{k}\left(E_{h}\right)$

$$
a_{h}(p, v)=l(v) .
$$

The NIPG method by Riviére and Wheeler employs the additional penalty term

$$
\mathrm{J}_{0}^{\sigma, \beta}(\mathrm{u}, v)=\sum_{\Gamma_{\mathrm{ef}} \in \Gamma_{\text {int }}} \frac{\sigma_{\mathrm{ef}}}{\left|\Gamma_{\mathrm{ef}}\right|^{\beta}} \int_{\Gamma_{\mathrm{ef}}}[\mathrm{u}][v] \mathrm{d} s+\sum_{\Gamma_{\mathrm{e}} \in \Gamma_{w \mathrm{~d}}} \frac{\sigma_{e}}{\left|\Gamma_{e}\right|^{\beta}} \int_{\Gamma_{\mathrm{e}}} \mathrm{u} v \mathrm{ds} .
$$

with user-defined parameters $\sigma$ and $\beta$; this penalty was employed in the original IP method. The formulation of the NIPG method is: Find $\mathrm{p} \in \mathrm{V}^{\mathrm{k}}\left(\mathrm{E}_{\mathrm{h}}\right)$ such that for all $v \in \mathrm{V}^{\mathrm{k}}\left(\mathrm{E}_{\mathrm{h}}\right)$

$$
a_{h}(p, v)+J_{0}^{\sigma, \beta}(p, v)=l(v)+\sum_{\Gamma_{e} \in \Gamma_{w d}} \frac{\sigma_{e}}{\left|\Gamma_{e}\right|^{\beta}} \int_{\Gamma_{e}} v p d s .
$$

Both the Baumann-Oden method and the NIPG method are nonsymmetric, even if the underlying continuous problem is symmetric. The Baumann-Oden method requires $k \geq 2$ to be stable, for the NIPG method $k \geq 1$ is sufficient. Both methods are locally conservative. This can be verified for the Baumann-Oden method by inserting a test function that is constant on each element into 4.20,

$$
\sum_{\Gamma_{e f} \in \Gamma_{\text {int }}} \int_{\Gamma_{\mathrm{ef}}}[v]\left\langle\rho \frac{\mathrm{K}}{\mu} \nabla p \cdot \mathbf{n}\right\rangle \mathrm{d} s+\sum_{\Gamma_{e} \in \partial \Omega} \int_{\Gamma_{\mathrm{e}}} \rho \frac{\mathrm{K}}{\mu} \nabla p \cdot \mathbf{n} v \mathrm{~d} s=\sum_{\mathrm{e} \in \mathrm{E}_{\mathrm{h}}} \int_{\Omega_{\mathrm{e}}} \mathrm{q} v \mathrm{~d} \boldsymbol{x},
$$

which reduces 4.20 to a flux balance equation. The close relation of the DG schemes to finite volume methods is apparent.

The Baumann-Oden DG method exhibits optimal order convergence in the $\mathrm{H}^{1}$-norm, i. e. $\mathcal{O}\left(\mathrm{h}^{\mathrm{k}}\right)$ for polynomials of degree $k$, provided that the solution is sufficiently regular. 
Convergence in $L_{2}$ is $\mathcal{O}\left(h^{k+1}\right)$ for $k$ odd and (suboptimal) $\mathcal{O}\left(\mathrm{h}^{k}\right)$ for $k$ even for the BaumannOden DG method. The NIPG is optimal in the $L_{2}$ norm for odd and even choices of $k$. The suboptimal convergence of the Baumann-Oden method is due to the absence of a stabilizing penalty term.

We prefer the Baumann-Oden DG method over the NIPG method because of its lack of user-defined parameters, because it is computationally cheaper, and because the suboptimal convergence in the $\mathrm{L}_{2}$ norm does not harm the efficiency of the method for groundwater flow equations, where the gradient of $p$ - measured in the $\mathrm{H}^{1}$ norm - is the relevant quantity.

The DG method can be extended to handle lower-dimensional elements and hence fractures in a similar way as described for the finite volume method. This would require the implementation of degrees of freedom on these boundary sides which are part of the fracture network. Another interesting potential of the method is the choice of basis functions for volumetric fracture elements, which are adjusted to the anisotropy and contain more degrees of freedom in one direction than in the other.

\subsection{Multigrid Solution}

After inserting a basis into (4.20), a large system of linear equations has to be solved. The multigrid method, explained in section 3.8 , can be used to solve these systems with optimal complexity.

We assume a hierarchy of nested triangulations $E_{0}, \ldots, E_{L}$, with $n_{l}$ elements on grid level $l$. On every grid level the discrete spaces $V_{l}^{k}=V^{k}\left(E_{l}\right)$ is equipped with the basis

$$
\mathcal{B}_{l}=\left\{\varphi_{1}^{l}, \ldots, \varphi_{\mathfrak{n}_{l}}^{l}\right\} .
$$

$\varphi_{i}^{l}$ has support in exactly one element. The indices of the basis functions associated with element $\Omega_{e}$ are

$$
\mathrm{I}_{e}^{l}=\left\{\mathrm{j} \mid \operatorname{supp} \varphi_{j}^{l} \subseteq \overline{\Omega_{e}}\right\} .
$$

Since the triangulations are nested, the according discrete spaces are also nested,

$$
\mathrm{V}_{0}^{\mathrm{k}} \subseteq \cdots \subseteq \mathrm{V}_{\mathrm{L}}^{\mathrm{k}}
$$

and coarse grid basis functions can be represented in the fine grid basis functions

$$
\varphi_{i}^{l}=\sum_{j=1}^{n_{l+1}} \omega_{i j}^{l+1} \varphi_{j}^{l+1} .
$$

The factors $\omega_{i j}^{l+1}$ can be computed as follows. Let $\varphi_{i}^{l}$ be the basis function with support in $\Omega_{e}, e \in \mathrm{E}_{l}$ and $\Omega_{f}$ be an element obtained from the subdivision of $\Omega_{e}$. By $\hat{\varphi}_{i}^{l}$ and $\hat{\varphi}_{j}^{l+1}$ we denote basis functions on the reference element that are mapped to the global basis functions $\varphi_{i}^{l}$ and $\varphi_{j}^{l+1}$. The restriction of $\varphi_{i}^{l}$ to $\Omega_{f}$ is a polynomial of degree $k$ and can be represented uniquely with the basis functions on $\Omega_{\mathrm{f}}$. The representation can be computed on the reference element $\hat{\Omega}$ using 
(4.28) $\left.\quad \varphi_{i}^{\mathrm{l}}\right|_{\Omega_{f}}=\sum_{j \in I_{f}^{l+1}} \omega_{i j}^{l+1} \varphi_{j}^{l+1}$

$$
\begin{gathered}
\Leftrightarrow \quad \hat{\varphi}_{i}^{l} \circ \mathrm{T}_{\Omega_{e}}^{-1} \circ \mathrm{T}_{\Omega_{f}}=\sum_{j \in \mathrm{I}_{f}^{l+1}} \omega_{i j}^{l+1} \hat{\varphi}_{j}^{l+1} \\
\Leftrightarrow \quad \forall m \in \mathrm{I}_{f}^{l+1}: \quad\left(\hat{\varphi}_{i}^{l} \circ \mathrm{T}_{\Omega_{e}}^{-1} \circ \mathrm{T}_{\Omega_{f}}, \hat{\varphi}_{m}^{l+1}\right)_{\hat{\Omega}}=\sum_{j \in \mathrm{I}_{f}^{l+1}} \omega_{i j}^{l+1}\left(\hat{\varphi}_{j}^{l+1}, \hat{\varphi}_{m}^{l+1}\right)_{\hat{\Omega}} .
\end{gathered}
$$

Here $(\cdot, \cdot)_{\hat{\Omega}}$ is the $\mathrm{L}_{2}$ scalar product and $T_{\Omega_{f}}$ is the mapping from the reference element to $\Omega_{\mathrm{f}}$. We can now employ the $\mathrm{L}_{2}$ orthonormality of the basis, due to which the mass matrix is the identity, and get

$$
\omega_{i j}^{l+1}=\left(\hat{\varphi}_{i}^{l} \circ T_{\Omega_{e}}^{-1} \circ T_{\Omega_{f}}, \hat{\varphi}_{m}^{l+1}\right)_{\hat{\Omega}} .
$$

The factors $\omega_{i j}^{l+1}$ can be precomputed, because the transformation $T_{\Omega_{e}}^{-1} \circ T_{\Omega_{f}}: \hat{\Omega} \rightarrow \hat{\Omega}$ depends only on the type of refinement and not on the shape of individual elements. However, since a large number of refinement rules are necessary for hybrid grids in three dimensions, we evaluate 4.29 using numerical quadrature. The factors $\omega_{i j}^{l}$ are the entries of the restriction and prolongation matrices.

The smoother should remove all algebraic errors that are not handled by the coarse grid correction. In that sense it is complementary to the coarse grid correction. The point-wise Jacobi and Gauß-Seidel iterations are not applicable since the matrix $A$ is only positive semidefinite $\left(x^{\top} A x \geq 0\right)$. Zero diagonal elements are obtained for inserting into the bilinear form a function that is constant on an element. For this reason one has to use block iterations. The most natural block structure is given by combining all degrees of freedom corresponding to one element

$$
A^{l, e, f}=\left(A_{i j}^{l}\right)_{i \in I_{e}^{l}, j \in I_{f}^{l}}
$$

and $A^{l}$ being the block matrix

$$
A^{l}=\left(A^{l, e, f}\right)_{\Omega_{e}, \Omega_{f}, e, f \in E_{l}} .
$$

Unfortunately, Jacobi and Gauß-Seidel iterations with respect to this block structure are not effective smoothers. This can be explained as follows: The diagonal block $A^{l, e, f}$ corresponds to a discretization of the flow equation on element $\Omega_{e}$ including a weak form of continuity of pressure over $\partial \Omega_{e}$. Because neighboring elements fix the "boundary conditions" for the current element, the error on the inter-element boundaries is only removed very slowly. The situation can be remedied by an overlapping patch smoother, where, for every element $\Omega_{e}$, all degrees of freedom of $\Omega_{e}$ and some neighborhood are updated. This iteration is an effective smoother but has a high operation count.

The incomplete LU-decomposition on the block structure is a very effective smoother and has a low operation count. The only drawback of ILU is that an additional matrix has to be stored. Note that the ordering of the blocks is not important unless some robustness with respect to discontinuous or anisotropic permeability is required. 
If the permeability $K$ is very different from element to element we observe the following problem: Assume that element $\Omega_{e}$ has permeability $\mathrm{K}=\epsilon \mathrm{I}$ and all neighboring elements $\Omega_{f}$ have permeability $\mathrm{K}=\mathrm{I}$ where $\mathrm{I}$ is the identity. Then all entries of the diagonal block $A^{\mathrm{l}, e, e}$ will have size $\mathcal{O}(\epsilon)$ and all off-diagonal blocks $A^{l, e, f}$ will have entries of size $\mathcal{O}(1)$. The patchwise smoothers do not work in this case. The ILU-smoother works if blocks corresponding to low permeability elements are ordered first. Therefore, for general permeability fields, we order the blocks with respect to increasing permeability.

Choosing $k$ equal for all grid levels is not necessary; the low-frequency errors on the coarser levels can be represented with polynomials of lower order. On the other hand, an implementation with equal polynomial degree on all grid levels is easier to implement and does not harm the overall performance.

Recently, a different approach to multigrid for the Baumann-Oden method has been proposed by Hemker et al. (2002b), Hemker et al. (2002a), Hemker and van Raalte (2002), for which convergence of the multigrid method with Jacobi and Gauß-Seidel smoothers could be shown for the Poisson equation in one and two space dimensions. They employed a point-wise block-partitioning, which gives better results than the cell-wise partitioning.

\subsection{Numerical Results for the Multigrid Solution of Discontinuous Galerkin Methods}

We investigate the efficiency of the multigrid algorithm for the Baumann-Oden method on several examples, where we compare them to the cell-centered finite volume method. The examples are chosen as a problem with full regularity, a problem with a reentrant corner and a problem with a heterogeneous permeability field with discontinuous coefficients. More applications, including unstructured grids in a complex geometry, are considered in Bastian and Reichenberger (2000). The last example is a comparison of the Baumann-Oden DG method and the finite volume method for a problem with a fracture. The fracture is modeled as a thin layer with different material properties.

We refrain from a description of the cell-centered finite volume method for single phase flow. The solution of the single phase flow equation would be possible with the finite volume method for two-phase flow, if $S_{g}=0$ is chosen throughout the domain. Due to its simplicity we implemented the finite volume scheme for single phase flow in porous media as well as in fractured porous media with mixed-dimensional elements.

\subsection{Single Phase Flow in Homogeneous Media}

The first example treats a problem with full regularity. For these kinds of problems the higher-order DG method should be clearly superior to the finite volume method. We solve the Poisson equation on the unit square

$$
-\Delta p=f \text { in } \Omega=(0,1)^{2} \quad p=p_{0} \text { on } \partial \Omega
$$

and choose $f$ and $p_{0}$ such that the exact solution

$$
p(x, y)=e^{-\left((x-1 / 2)^{2}+(y-1 / 2)^{2}\right)}
$$




\begin{tabular}{lrrrrrr}
\hline $\mathrm{h}^{-1}$ & $\mathrm{FV}$ & $\mathrm{r}=2$ & $\mathrm{r}=3$ & $\mathrm{r}=4$ & $\mathrm{r}=5$ & $\mathrm{r}=6$ \\
\hline 4 & 3 & 5 & 5 & 5 & 5 & 4 \\
8 & 4 & 7 & 6 & 6 & 5 & 6 \\
$\mathrm{I} 6$ & 4 & 7 & 6 & 6 & 5 & 6 \\
32 & 4 & 7 & 6 & 6 & 5 & 6 \\
64 & 4 & 7 & 6 & 6 & 5 & 6 \\
$\mathrm{I} 28$ & 4 & 6 & 6 & 6 & & \\
256 & 4 & & & & & \\
$5 \mathrm{I} 2$ & 4 & & & & & \\
\hline
\end{tabular}

Table 4.2.: Number of multigrid iterations for the fully regular model problem.

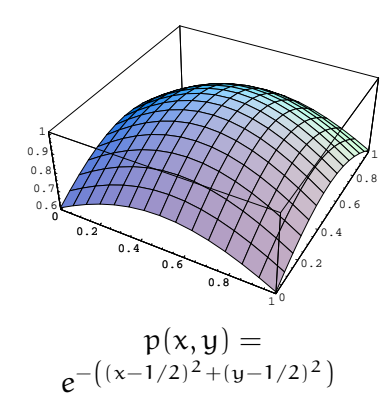

is obtained. In figure 4.2 the error in the $\mathrm{L}_{2}$ and $\mathrm{H}^{1}$ norms are plotted. Note that the $x$-axis does not show the necessary number of iterations but the wall clock computation time, which is the more interesting quantity in practice. The number of multigrid iterations are displayed in table 4.2 , which shows the $h$-independence of the method. We compared the finite volume method (FV) and the DG method with polynomials up to degree six for a multigrid V-cycle with one ILU pre- and post-smoothing step and list iteration numbers for a $10^{-8}$ reduction of the initial residual.

The DG method is clearly superior for this example. The suboptimal convergence $\mathcal{O}\left(\mathrm{h}^{\mathrm{k}}\right)$ in the $\mathrm{L}_{2}$ norm for even degrees $k$ is confirmed by the numerical experiment. For odd polynomial degrees $k$ the convergence is optimal $\left(\mathcal{O}\left(h^{k+1}\right)\right)$ in the $L_{2}$ norm, and optimal convergence of $\mathcal{O}\left(h^{k}\right)$ in the $H^{1}$-norm is obtained for all degrees of $k$.

\subsubsection{Single Phase Flow in Heterogeneous Media}

The second example is taken from Durlofsky (I994). It explores the quality of the DG solutions for elliptic problems with highly discontinuous coefficients for the problem

$$
-\nabla \cdot(K \nabla p)=f \quad \text { in } \Omega=(0,1)^{2} .
$$

The boundary conditions are chosen as $p=1$ for $x=0, p=0$ for $x=1$ and no flow boundary conditions for $y=0$ and $y=1$. The permeability field is defined on a regular $20 \times 20$ mesh and is shown in Fig. 4.3 on the left. In dark areas the permeability is $\mathrm{K}=10^{-6} \cdot \mathrm{I}$, elsewhere it is $\mathrm{K}=\mathrm{I}$. We employ a coarse grid with $20 \cdot 20 \cdot 2$ triangular elements. The coarse grid resolves the discontinuities in the permeability field, finer grids are obtained by regular refinement. Fig. 4.3 shows on the right the flow field computed with degree $k=3$ on the coarsest mesh.

Durlofsky (I994) gave a reference solution for the total flux through the system, based on computing approximations on a sequence of meshes up to $200 \times 200$ with a cell centered 
4. The Discontinuous Galerkin Method for Elliptic problems
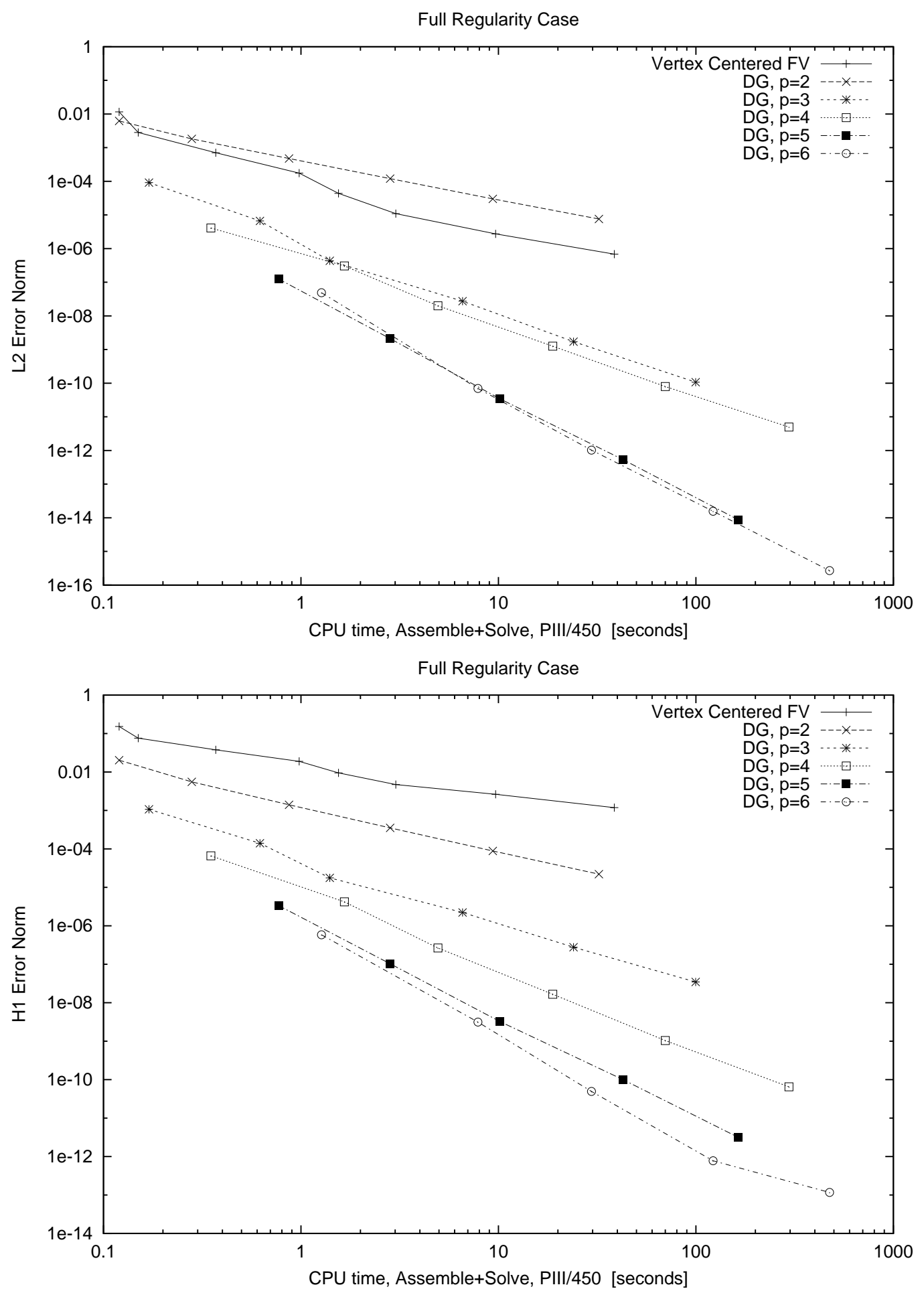

Figure 4.2.: $\mathrm{L}_{2}$ and $\mathrm{H}^{1}$ error for the full regularity problem. 

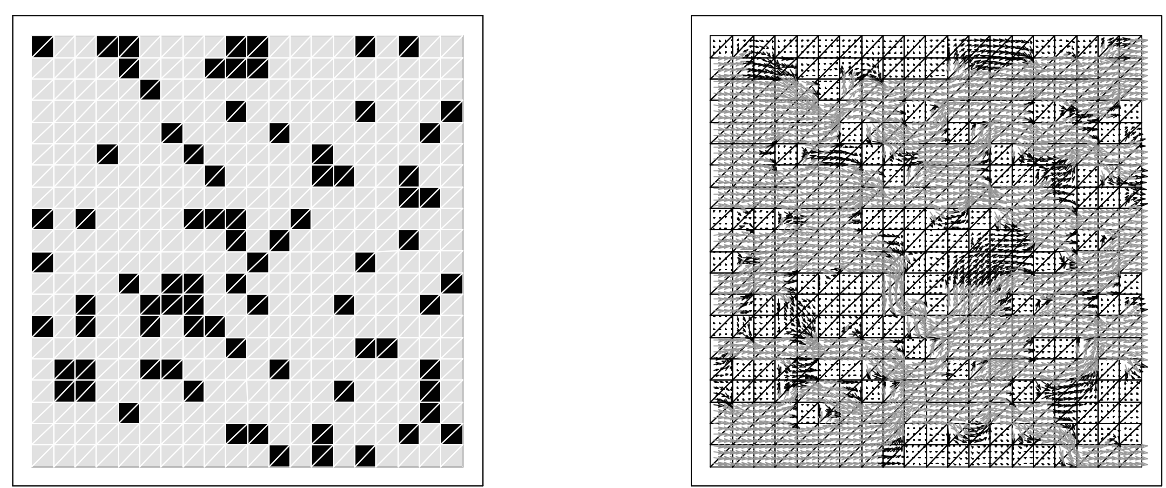

Figure 4.3.: Permeability and flow field for the discontinuous coefficient example computed with DG(3). Permeability 1 is shown in light gray and $10^{-6}$ in black. Vectors not drawn to scale are indicated by gray color in the vector plot.

\begin{tabular}{lrrrrrrr}
\hline $\mathrm{h}^{-1}$ & $\mathrm{FV}$ & $\mathrm{k}=2$ & $\mathrm{k}=3$ & $\mathrm{kk}=4$ & $\mathrm{k}=5$ & $\mathrm{k}=6$ & $\mathrm{MFE}$ \\
\hline 20 & $0.699 \mathrm{I}$ & 0.5094 & 0.5152 & 0.5174 & 0.5232 & 0.5152 & 0.4508 \\
40 & 0.6466 & 0.5179 & $0.518 \mathrm{I}$ & 0.5208 & 0.5206 & & \\
80 & 0.6170 & 0.5194 & 0.5192 & $0.520 \mathrm{I}$ & & & \\
160 & 0.5998 & 0.5199 & 0.5198 & & & & \\
320 & 0.5890 & & & & & & \\
640 & 0.5816 & & & & & & \\
\hline
\end{tabular}

Table 4.3.: Total flux through the system for discontinuous coefficient example.

\begin{tabular}{rlrrrr}
\hline$l$ & $\mathrm{~h}^{-1}$ & FV & $k=2$ & $k=3$ & $k=4$ \\
\hline $\mathrm{I}$ & 40 & 6 & $\mathrm{I} 4$ & $\mathrm{I} 4$ & $\mathrm{I} 6$ \\
2 & 80 & 7 & $\mathrm{I} 4$ & $\mathrm{I} 2$ & $\mathrm{I} 5$ \\
3 & $\mathrm{I} 60$ & 7 & $\mathrm{I} 3$ & $\mathrm{I} 2$ & \\
4 & 320 & 8 & & & \\
5 & 640 & 9 & & & \\
\hline
\end{tabular}

Table 4.4.: Number of multigrid cycles in the discontinuous coefficient example.

finite volume scheme and extrapolation to $h=0$. The "exact" value is 0.5205 . We compare the vertex centered finite volume method-which in this case is identical to PI conforming finite elements - and DG with $k=2, \ldots, 6$. The values for the mixed finite element method are taken from Durlofsky (1994). The results show that the conforming finite volume/finite element method is not well suited for this problem. The error in the mixed finite element solution on the coarsest mesh is approximately a factor six larger than the error in the DG 

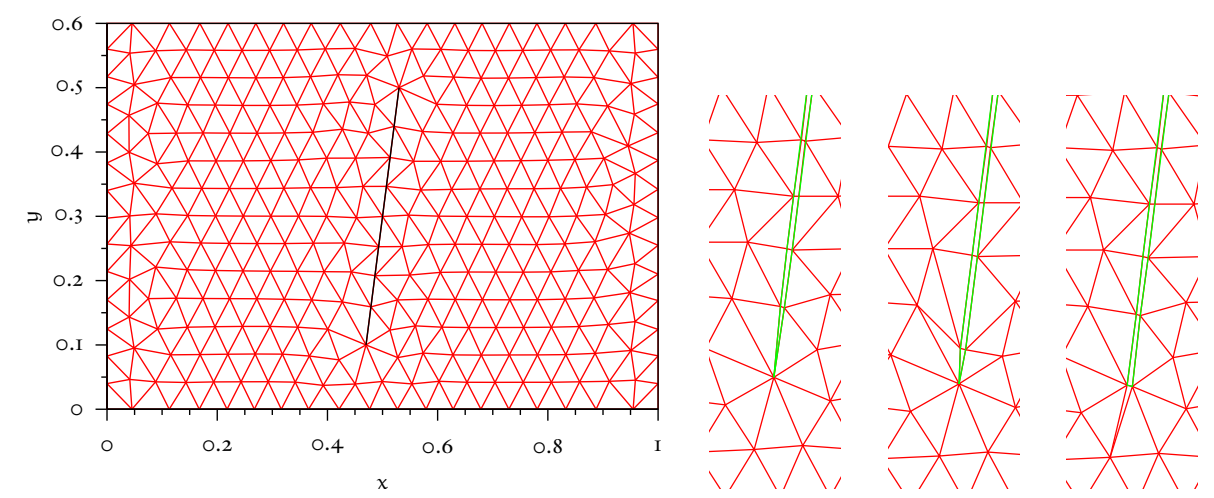

Figure 4.4.: Domain with one fracture. On the left is the domain with the one-dimensional fracture, on the right grid details of the volumetric fracture ends 13, 13h, 13q.

result on the same mesh, but we note that the number of unknowns for the DG method (4800) is 2.4 times larger than the number of unknowns for the mixed finite element method (2000). The mixed finite element method is considered optimal for this kind of problem, and the results of the DG method show that it is very well suited for this problem.

The performance of the multigrid method for the discontinuous coefficient example is shown in table 4.4. We use a multigrid $V$-cycle with $v_{1}=v_{2}=2$ ILU smoothing steps as a preconditioner in the Bi-CGSTAB-method (van der Vorst, 1992). The table shows the number of preconditioner evaluations needed to reduce the norm of the defect by $10^{-8}$. Again the iteration numbers seem to be independent of $h$ and $k$.

\subsection{Single Phase Flow in Fractured Media}

The third example considers a domain with a single fracture. We compare the behavior of the DG method with the behavior of a vertex-centered finite volume method which discretizes fractures either by one-dimensional or two-dimensional elements. The grids with the volumetric mesh were created from the coarse grid of the domain denoted by $13-1 \mathrm{~d}$ with the one-dimensional fracture by the methods described in Neunhäuserer (2002). The coarse grid for domain $13-1 \mathrm{~d}$ is shown in figure 4.4 . There are three variants of the volumetric fracture: In two domains 13 and $13 \mathrm{~h}$ the fracture has triangular ends, 13q has quadrilateral elements throughout the whole fracture. In domain $13 \mathrm{~h}$ the fracture end triangles were reduced in length to enlarge the very small angle at the fracture end.

In the domains we solve the groundwater equation

$$
\begin{aligned}
-\nabla \cdot\left(\rho \frac{\mathrm{K}}{\mu} \nabla p\right) & =\rho \mathrm{q} & & \text { in } \Omega . \\
p & =10^{5}[\mathrm{~Pa}] & & \text { on } 0 \times[0,0.6] \\
p & =0.999 \cdot 10^{5}[\mathrm{~Pa}] & & \text { on } 0 \times[1,0.6]
\end{aligned}
$$




\subsection{Single Phase Flow in Fractured Media}

Comparison of pressure for DG in different domains

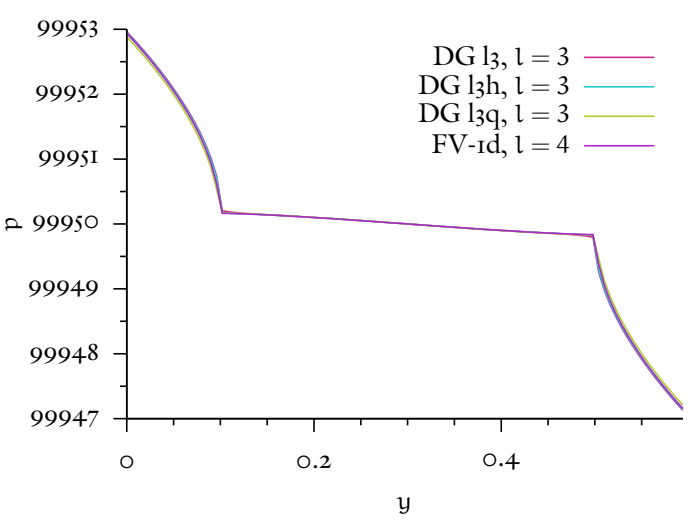

Comparison of pressure for $\mathrm{FV}$ in different domains

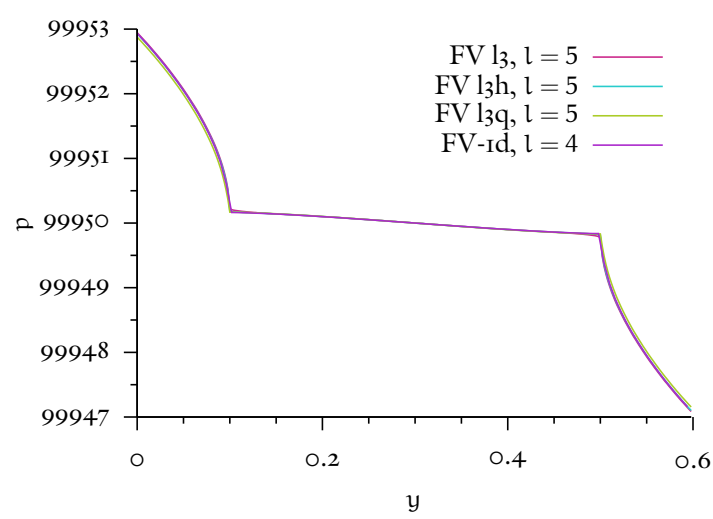

Comparison of $|v|$ for DG in different domains
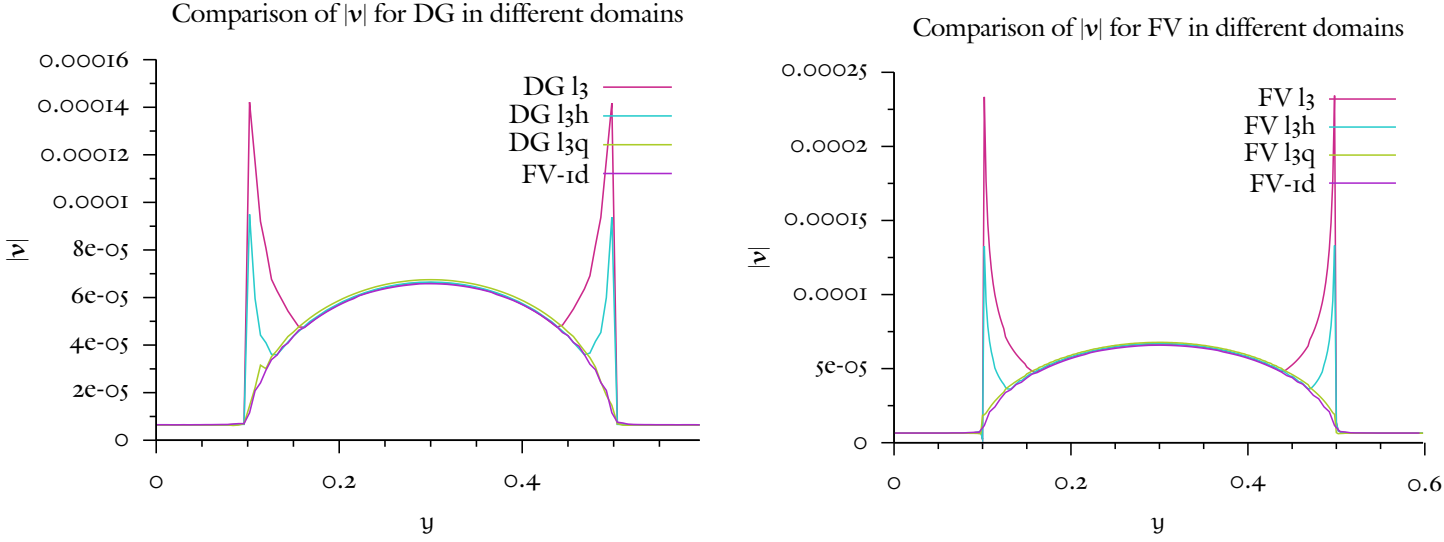

Figure 4.5.: Pressure distribution along a line through the fracture for different fracture realizations (above) and the corresponding absolute value of the velocity.

with parameters

$$
\begin{aligned}
\rho & =1000\left[\mathrm{~kg} / \mathrm{m}^{3}\right] & \mu & =0.0013[\mathrm{~kg} /(\mathrm{ms})] \\
\mathrm{K}^{\mathrm{m}} & =8.3333 \cdot 10^{-11}\left[\mathrm{~m}^{2}\right] & \mathrm{K}^{\mathrm{f}} & =8.3333 \cdot 10^{-8}\left[\mathrm{~m}^{2}\right] \\
\mathrm{b} & =5 \cdot 10^{-3}[\mathrm{~m}] & &
\end{aligned}
$$

As in the previous chapters we denote quantities in the matrix with superscript $m$ and quantities in the fracture with superscript $f$.

Numerical solutions to 4.33 are obtained by the methods:

FV-1d The finite volume method with one-dimensional fractures.

FV The finite volume method with two-dimensional fractures.

DG The Baumann-Oden DG method with two-dimensional fractures. The choice of basis functions is indicated by $P_{k}$ and $Q_{k}$. 


\section{The Discontinuous Galerkin Method for Elliptic problems}
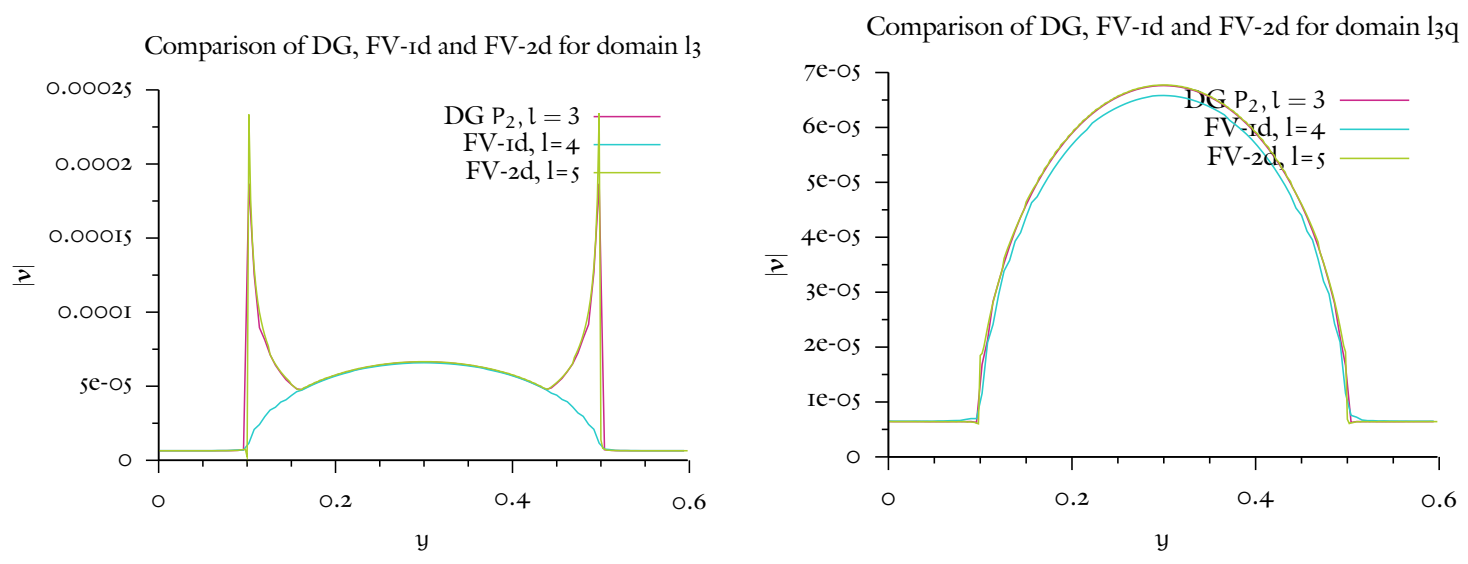

Figure 4.6.: Comparison of the DG, FV and FV-Id method.

The number of unknowns for the different meshes on level $l$ are:

\begin{tabular}{rrrrrr}
\hline $\mathrm{l}$ & $\mathrm{FV}-1 \mathrm{~d}$ & $\mathrm{FV}$ & $\mathrm{DG}-\mathrm{P}_{2}$ & $\mathrm{DG}_{3} \mathrm{P}_{3}$ & $\mathrm{DG} \mathrm{Q}_{2}$ \\
\hline $\mathrm{O}$ & 305 & $3 \mathrm{I} 2$ & 3300 & 5500 & 4950 \\
$\mathrm{I}$ & $\mathrm{II} 5 \mathrm{I}$ & $\mathrm{II} 79$ & $\mathrm{I3200}$ & 22000 & 19800 \\
2 & 4469 & $458 \mathrm{I}$ & 52800 & 88000 & 79200 \\
3 & $\mathrm{I} 7609$ & $\mathrm{I} 8057$ & $2 \mathrm{II} 200$ & 352000 & $3 \mathrm{I} 6800$ \\
4 & 69905 & 71697 & & & \\
5 & & 285729 & & & \\
\hline
\end{tabular}

For the finite volume methods, the number of unknowns is equal to the number of nodes in the grid, for the DG method the number of unknowns is equal to the number of elements times the dimension of the space of basis functions.

The comparison of the method is done by extracting the values along the line from $(0.455,0)$ to $(0.545,0.6)$ - this is the line along the fracture middle surface. Along this line we compare the pressure $p$ and the absolute value of the Darcy velocity, $|v|$.

In figure 4.5 we compare how the different fracture realizations-one-dimensional, with triangles and with quadrilaterals - affect the solution. The figure shows on the left the results for the DG method with $P_{k}$ on level 3 and the FV-1d method on level 4. On the right the results for the FV method on level 5 are compared to the FV-1d method on level 4 . The differences in the pressure are very small. We can get more insight from the plot of the absolute value of the Darcy velocity below. Here it is obvious that the choice for the fracture ends has a significant influence on the velocity distribution. While the realization with quadrilateral ends agrees nicely with the lower-dimensional approximation, the velocity in the region of the fracture end triangle on the coarsest mesh introduces an increase in the velocity toward the fracture end. The velocity peaks appear for the DG method and the FV method, for the FV method they are more developed. 

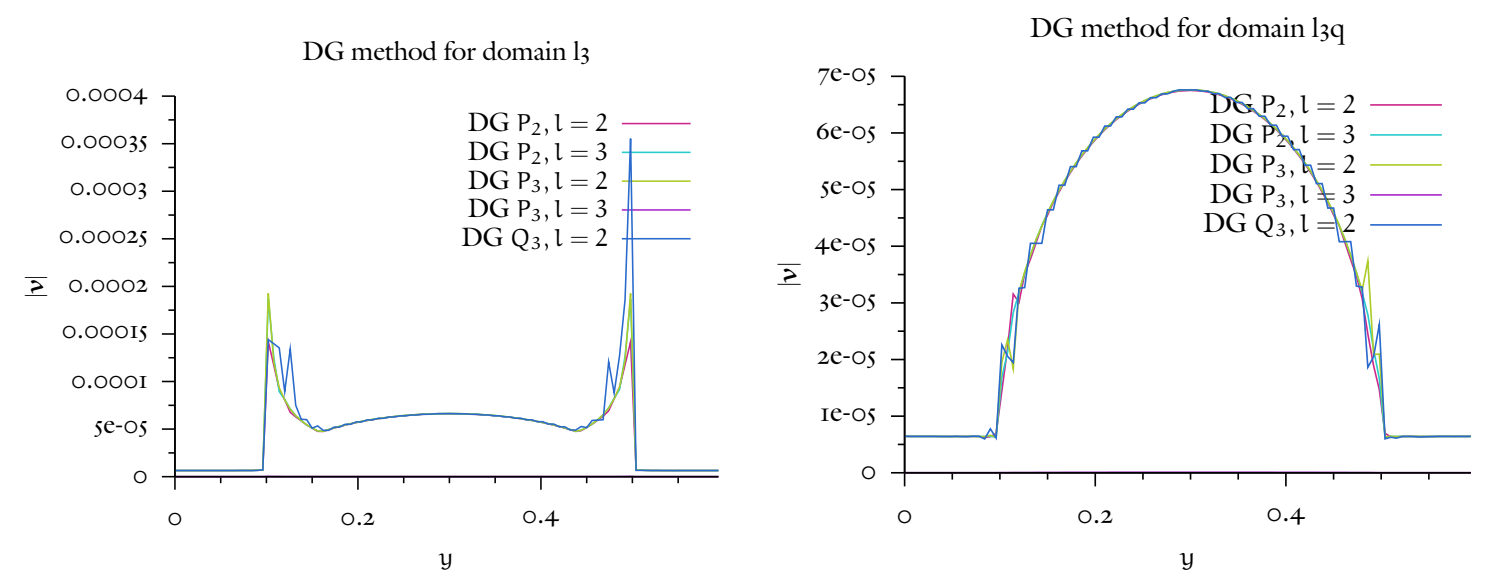

Figure 4.7.: $h$ and $p$ convergence of the DG method.

The discretization methods are compared once more in figure 4.6 , to make the difference between the methods more visible. The FV-1d method solution is included as a "reference" solution. In the case of the quadrilateral fracture ends, the FV method and the DG method solutions are almost identical. The DG method shows some minor discontinuities in the elements at the fracture ends. The FV-1d method has a smoother progression which is explainable because a node shared by a fracture and the matrix carries an averaging solution. Due to this averaging the total velocity in the fracture is smaller than for the other methods, which have degrees of freedom entirely inside the fracture.

Figure 4.7 compares DG solutions for $P_{2}$ and $P_{3}$ on grid levels 2 and 3, and for $Q_{3}$ on level 3. The $P_{2}$ solution on level 3 and the $P_{3}$ solution on level 2 are almost identical. The $\mathrm{Q}_{3}$ solution has strong oscillations in the region where triangular elements are employed; in the case of the quadrilateral fracture ends (13q) the oscillations are less pronounced. The quadrilateral case also shows non-monotone behavior for the methods with $k=3$, while the $k=2$ solutions are monotone. This suggests that the problem does not possess the required regularity near the fracture-matrix interface that makes higher order methods applicable.

Figure 4.8 shows development of sharp velocity peaks for the FV-2d method, which reach their maximum for level 4 and decay afterwards. They are not present in the case of the fracture with quadrilateral ends (as shown in figure 4.6). 
4. The Discontinuous Galerkin Method for Elliptic problems
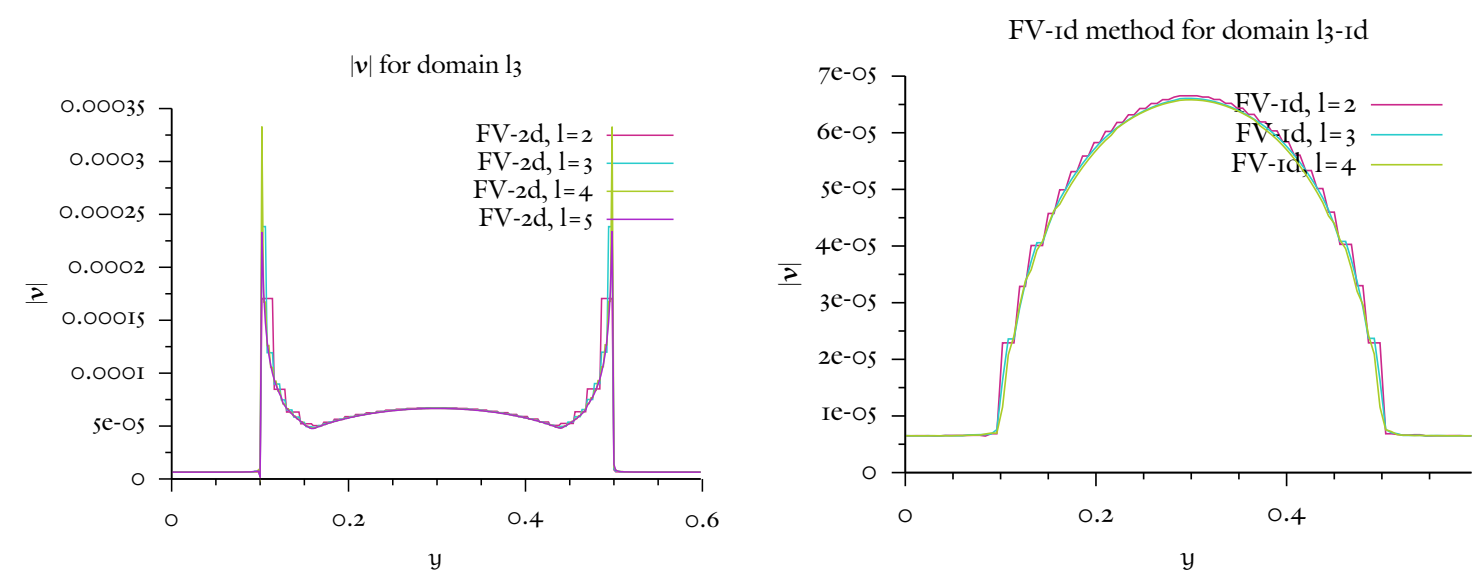

Figure 4.8.: Convergence of the FV-1d and FV-2d methods. 


\section{Numerical experiments}

\section{I. VERTICAL WATER-GAS FLOW}

The first numerical experiment with two-phase flow is set up to assess the difference between a lower-dimensional fracture approach and a fracture with volumetric elements. We choose a simple configuration with only one fracture in order not to include too many different effects into the solution. The setup includes one vertical fracture inside a domain of $1 \times 1[\mathrm{~m}]$, a sketch is displayed in figure 5.I. The fracture is located along the line from $(0.5,0.2)$ to $(0.5,0.8)$. We consider inflow of a compressible gas phase at the south boundary.

The parameters of the simulation are artificially chosen but give a representative picture of fracture-matrix interaction. The parameters are

$$
\begin{aligned}
\rho_{w} & =1000\left[\mathrm{~kg} / \mathrm{m}^{3}\right] & \rho_{\mathrm{g}} & =\frac{p_{\mathrm{n}}}{84149.6}\left[\mathrm{~kg} / \mathrm{m}^{3}\right] \\
\mu_{w} & =10^{-3}[\mathrm{~Pa} \mathrm{~s}] & \mu_{\mathrm{g}} & =1.65 \cdot 10^{-5}[\mathrm{~Pa} \mathrm{~s}] \\
\Phi^{f} & =0.3 & \Phi^{\mathrm{m}} & =0.1 \\
\mathrm{~K}^{f} & =10^{-8} & \mathrm{~K}^{\mathrm{m}} & =10^{-12} \\
S_{w r}^{f} & =0 & S_{w r}^{m} & =0 \\
S_{g r}^{f} & =0 & S_{g r}^{m} & =0 \\
\lambda^{f} & =2 & \lambda^{\mathrm{m}} & =2 \\
\lambda^{f} & =1000[\mathrm{~Pa}] & \lambda^{\mathrm{m}} & =2000[\mathrm{~Pa}]
\end{aligned}
$$
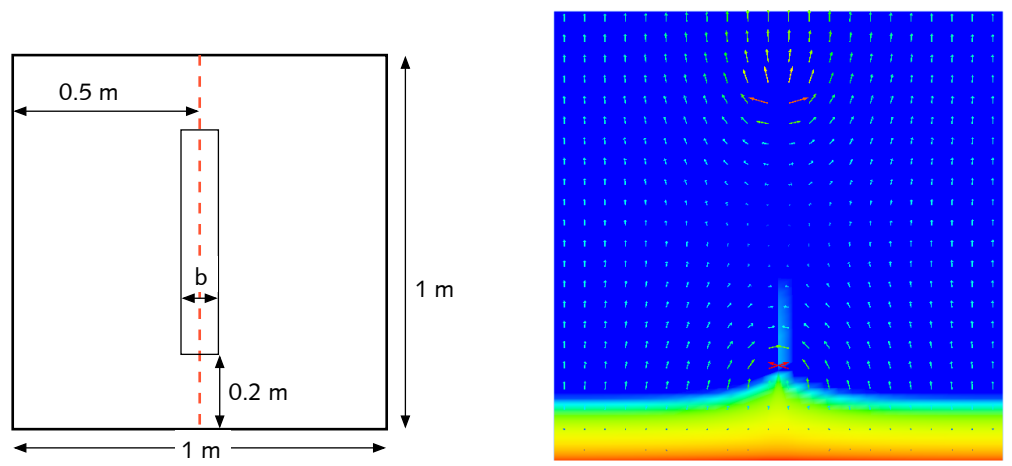

Figure 5.I.: Sketch of the domain for the vertical water-gas flow example and $S_{g}$ and $\mathbf{u}_{w}$ at $\mathrm{t}=70 \mathrm{~s}$. 


\section{NUMERICAL EXPERIMENTS}
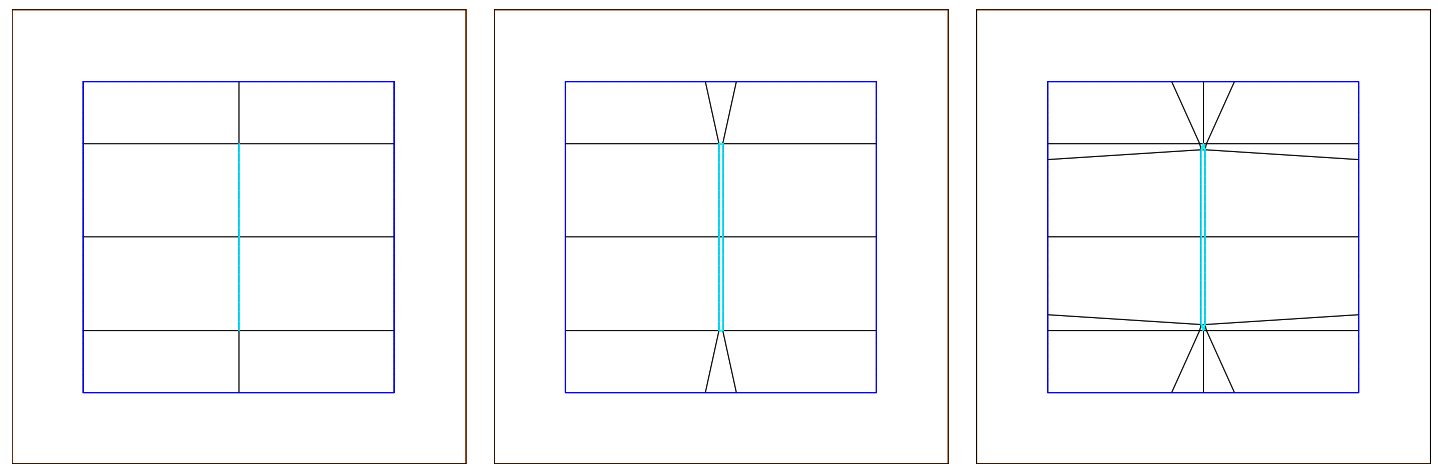

Figure 5.2.: Coarse grids of the vertical water-gas flow simulation for the one-dimensional fracture (left) and the two-dimensional fractures with rectangular ends (middle) and triangular ends (right).

The fracture width $b$ is chosen from $\{0.01,0.005,0.001\}[\mathrm{m}]$, to determine the influence of the fracture width on the solution and on the solution efficiency. Boundary conditions are $S_{g}=p_{w}=0$ on the north boundary, and Neumann boundary conditions elsewhere. At the south boundary the value of the Neumann boundary condition for the saturation is $-2.5 \cdot 10^{-5}\left[\mathrm{~kg} / \mathrm{m}^{2}\right]$, all remaining Neumann boundary condition values are 0 .

Without the fracture, the problem would be quasi one-dimensional, but a look at the flow field reveals how the influence of the fracture affects the solution behavior (figure 5.I, right). We choose a vertical fracture in order to exclude grid dependent phenomena from the experiment. In non-vertical fractures, the gas phase travels along a thin layer below the top fracture wall. This is the behavior that is to be expected in thin layers, but from thin open fractures we expect a different behavior because the strong capillary effects in the fracture create a different flow pattern. It must be doubted that the gas-water flow in fractures modeled with volumetric elements creates a physically relevant solution: The flow field developing in the 'additional' dimension is not supported by fracture flow models.

Three different coarse grids are employed, depicted in figure 5.2. The first grid (left) employs the mixed dimensional finite volume method and models the fracture by onedimensional elements. The other grids resolve the fracture with two-dimensional elements. The grid in the middle uses only quadrilateral elements, the grid on the right has a triangular element at both fracture ends. It should be obvious from this example that grid generation for fractures with volumetric elements is much more difficult than for lower-dimensional fractures.

For the simulation we employed the backward Euler scheme with fixed time steps. The nonlinear equations were solved with inexact Newton method with line search. The linear systems were solved with the $\mathrm{V}(2,2)$-cycle multigrid method with ILU smoothing, accelerated with Bi-CGSTAB. Additional properties of the solution process can be found in 

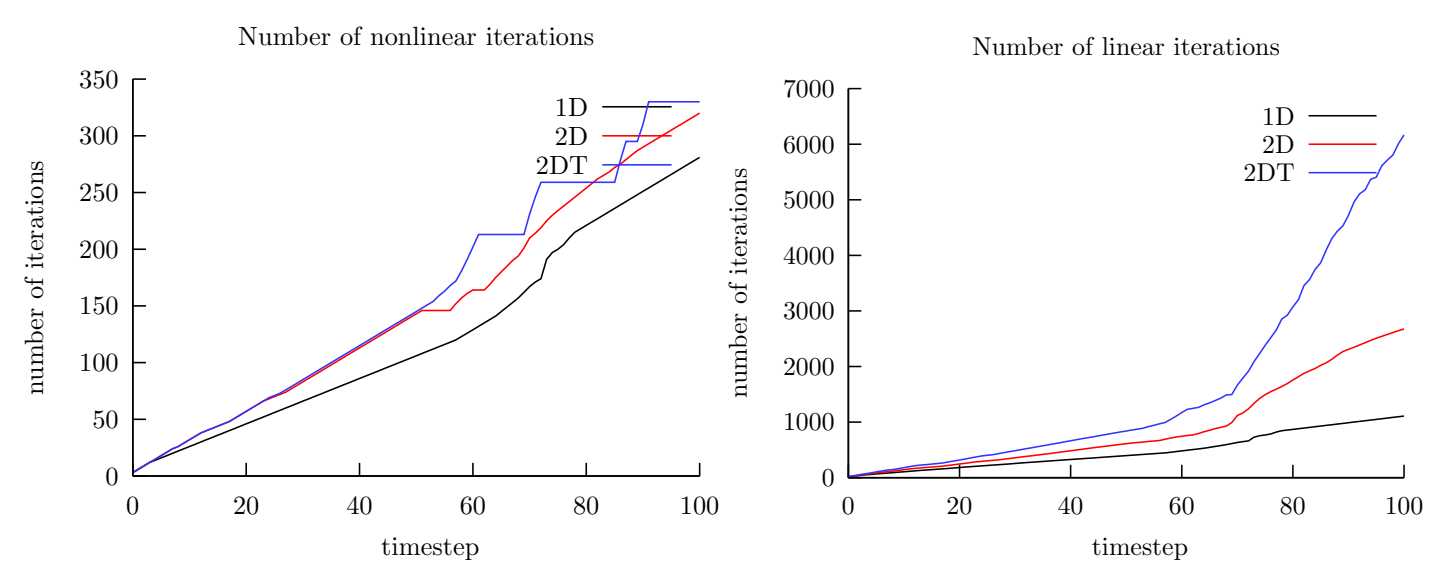

Figure 5.3.: Linear and nonlinear iterations for vertical infiltration problem with $b=0.005$ on level $l=3$.

table 5.1 .

We compared the discretization schemes by plotting the values for $S_{g}$ and $p_{w}$ at different time steps along the line from $(0.5,0)$ to $(0.5,1)$. The time steps are chosen as $t=$ $50 \mathrm{~s}, 74 \mathrm{~s}, 100 \mathrm{~s}$. At $\mathrm{t}=50 \mathrm{~s}$ the gas has not reached the fracture yet, at $\mathrm{t}=74 \mathrm{~s}$ some gas is inside the fracture and at $t=100 \mathrm{~s}$ the gas has reached the fracture end and has penetrated the matrix. The series of figures 5.4 5.6 reveals the slow grid convergence which is typical for gas-water problems. This is especially pronounced at $t=74 \mathrm{~s}$, where the fast movement in the fracture amplifies the effect. The plot at the top right of figure 5.5 shows a study on six of eight refinement levels (level 0 is the coarse grid). Only for this refinement can we start to speak of grid convergence.

The influence of the type of fracture realization (lower-dimensional, with quadrilateral or triangular fracture ends) has already been observed for single phase flow in chapter 4 . In

\begin{tabular}{|c|c|c|c|c|c|c|c|}
\hline \multirow[b]{2}{*}{ Level l } & \multirow[b]{2}{*}{$\Delta \mathrm{t}[\mathrm{s}]$} & \multicolumn{2}{|c|}{ one-dimensional } & \multicolumn{2}{|c|}{ two-dimensional } & \multicolumn{2}{|c|}{ two-dimensional, triangle } \\
\hline & & \#E & $\# \mathrm{~N}$ & \#E & $\# \mathrm{~N}$ & \#E & $\# \mathrm{~N}$ \\
\hline 2 & 2 & I53 & I28 & $22 \mathrm{I}$ & I92 & 384 & 377 \\
\hline 3 & I & $56 \mathrm{I}$ & $5 \mathrm{I} 2$ & 825 & 768 & I536 & I425 \\
\hline 4 & 0.5 & 2145 & 2048 & 3185 & 3072 & 6144 & 5537 \\
\hline 5 & 0.25 & 8385 & 8I92 & $\mathrm{I} 25 \mathrm{I} 3$ & I2288 & 24576 & 21825 \\
\hline 6 & 0.5 & 33153 & 32768 & & & & \\
\hline 7 & 0.25 & I3I $84 \mathrm{I}$ & I3IO72 & & & & \\
\hline
\end{tabular}

Table 5.I.: Time step $\Delta \mathrm{t}$, number of elements \#E and nodes \#N for grids of the vertical gas flow problem 
figure 5.8 we display the shape of the saturation curve for the three discretizations at $t=74 \mathrm{~s}$ and $t=100 \mathrm{~s}$. This is an interesting comparison, because it compares the mixed-dimensional model with a "trusted one", the model with only two-dimensional elements. First, we would like to point out that the total mass in the system is the same in all realizations. A contradicting impression could arise because the saturation curve resembles a one-dimensional model problem and seems to reveal that in the lower-dimensional case less mass is present. This is not the case-only the mass in the fracture is different.

Apparently, the fracture geometry has a notable influence on the solution. The difference is, however, small enough to be of relatively small significance when compared to other uncertainties associated with the modeling and simulation process.

If the differences in the solution are small, the differences in obtaining the solution are quite remarkable. In figure 5.3 we plot the number of linear multigrid cycles and nonlinear Newton steps necessary for the simulation with Ioo time steps. Note that in some cases the convergence requirements had to be relaxed if convergence could not be reached to the desired level. In these cases the counted total number of Newton steps is not increased, and this accounts for the behavior of the 2DT case. The systems in the lower-dimensional realization are easier to solve and require less iterations than the two-dimensional realization. The curves also reveal that the system solution only starts to get difficult when the gas has reached the fracture and the nonlinearities in the constitutive relations in combination with the discontinuous material properties make the systems more difficult to solve.

We include only one figure (5.7) with the values of $p_{w}$ at $t=74 \mathrm{~s}$ for all three fracture realizations, because the differences in the pressure are small and the interesting quantity is the gas saturation. 


\section{I. VERTICAL WATER-GAS FLOW}
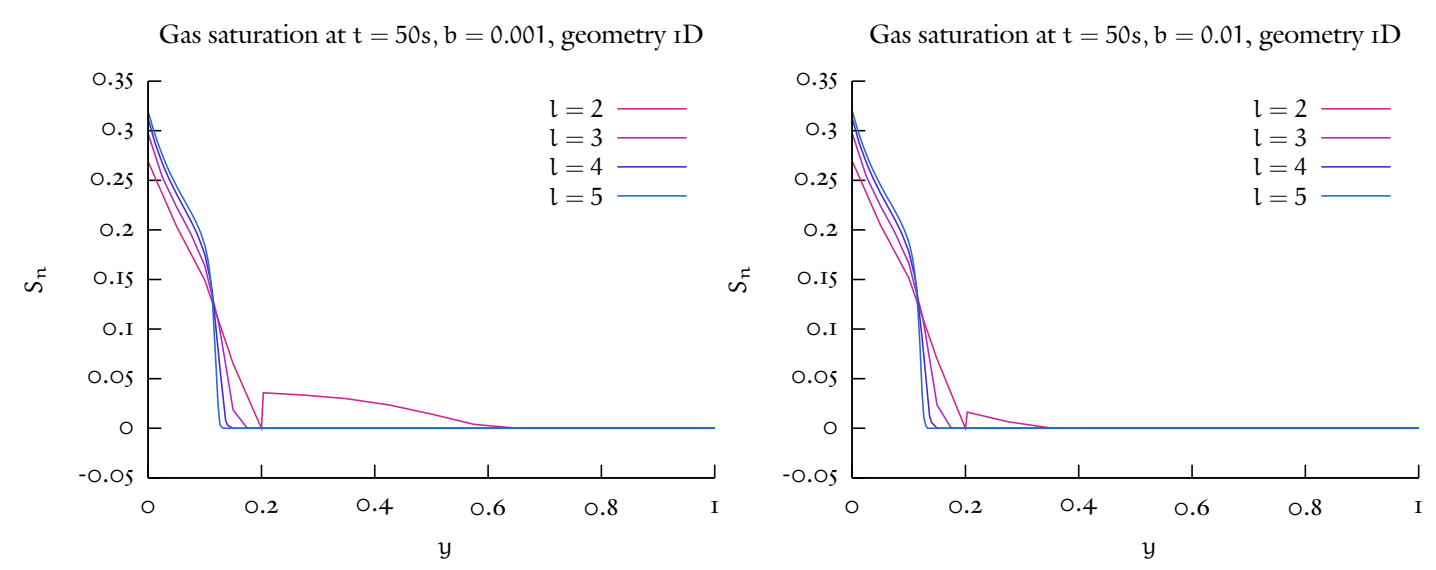

Gas saturation at $t=50 s, b=0.001$, geometry $2 \mathrm{D}$

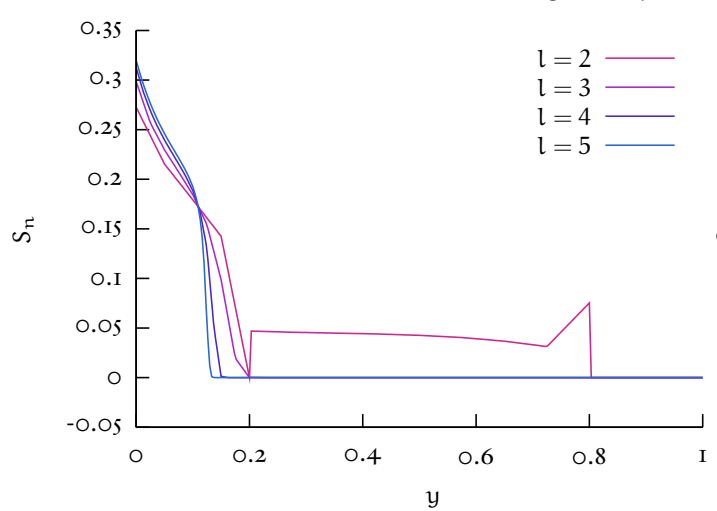

Gas saturation at $t=50 \mathrm{~s}, \mathrm{~b}=0.01$, geometry $2 \mathrm{D}$

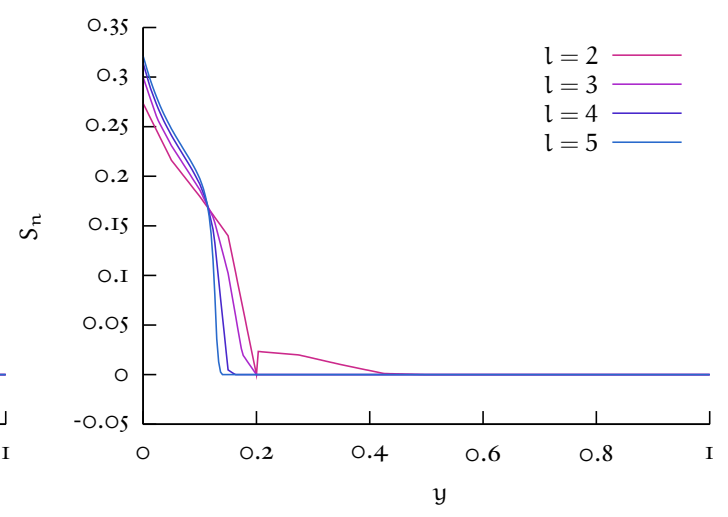

Gas saturation at $t=50 \mathrm{~s}, \mathrm{~b}=0.001$, geometry $2 \mathrm{DT}$

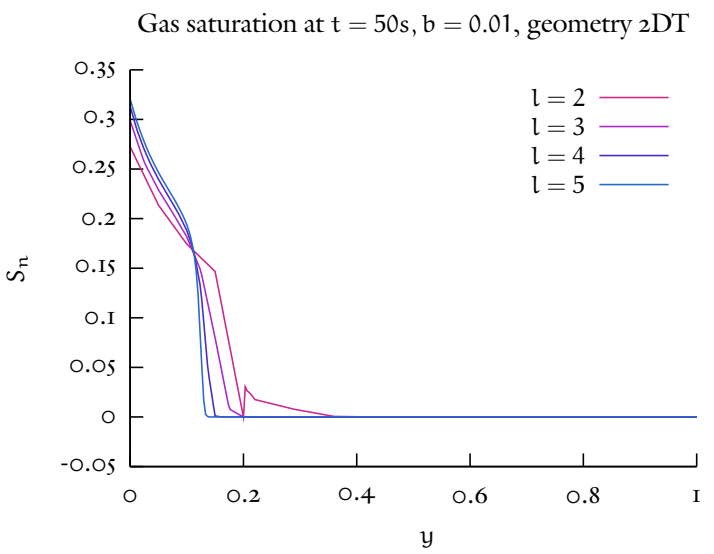

Figure 5.4.: Grid convergence of vertical gas-water flow test case for $t=50 \mathrm{~s}$. The gas saturation along the line in $y$-direction is plotted for grid level $l$ and the fracture widths $b=0.001$ (left) and $b=0.01$ (right). Results in the first row are for the onedimensional fracture geometry, the middle row for the two-dimensional fracture geometry with rectangular ends and the last row for the two-dimensional fracture geometry with triangular ends. 


\section{Numerical EXPERIMENTS}

Gas saturation at $\mathrm{t}=74 \mathrm{~s}, \mathrm{~b}=0.001$, geometry $\mathrm{ID}$

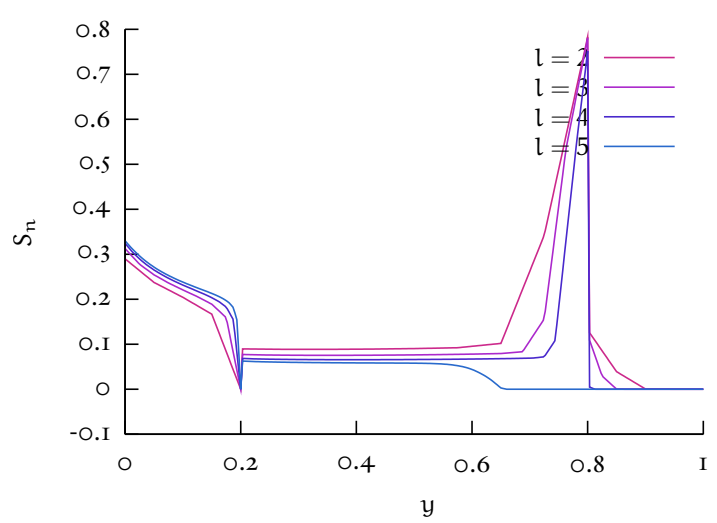

Gas saturation at $\mathrm{t}=74 \mathrm{~s}, \mathrm{~b}=0.001$, geometry $2 \mathrm{D}$

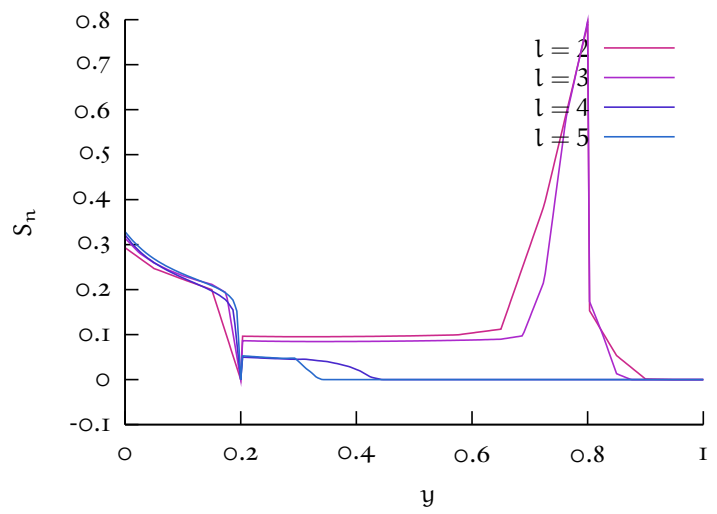

Gas saturation at $\mathrm{t}=74 \mathrm{~s}, \mathrm{~b}=0.001$, geometry $2 \mathrm{DT}$

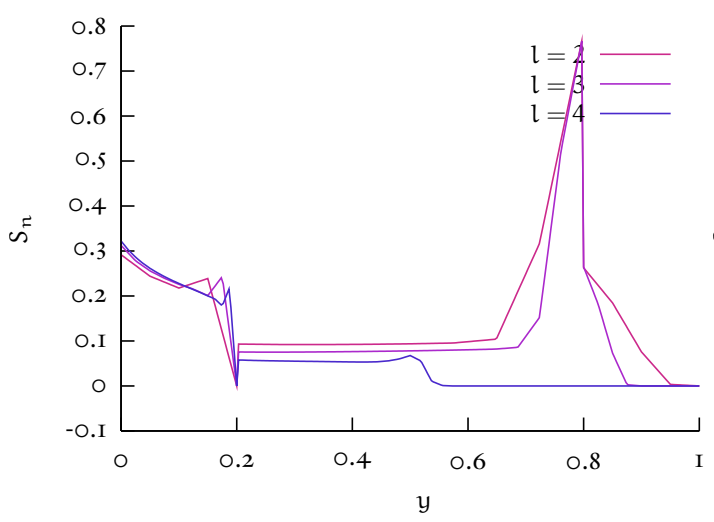

Gas saturation at $t=74 \mathrm{~s}, \mathrm{~b}=0.01$, geometry $\mathrm{ID}$

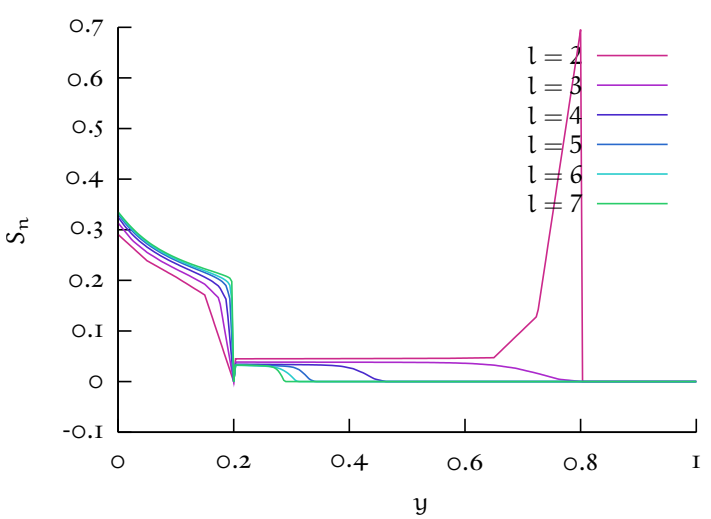

Gas saturation at $\mathrm{t}=74 \mathrm{~s}, \mathrm{~b}=0.01$, geometry $2 \mathrm{D}$

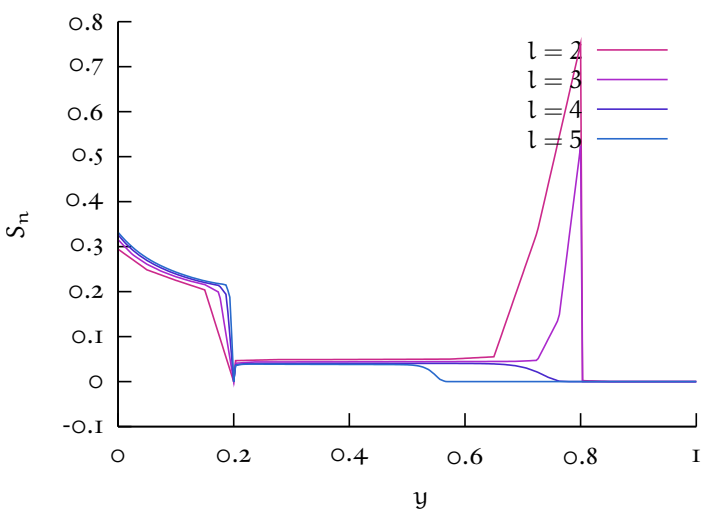

Gas saturation at $\mathrm{t}=74 \mathrm{~s}, \mathrm{~b}=0.01$, geometry $2 \mathrm{DT}$

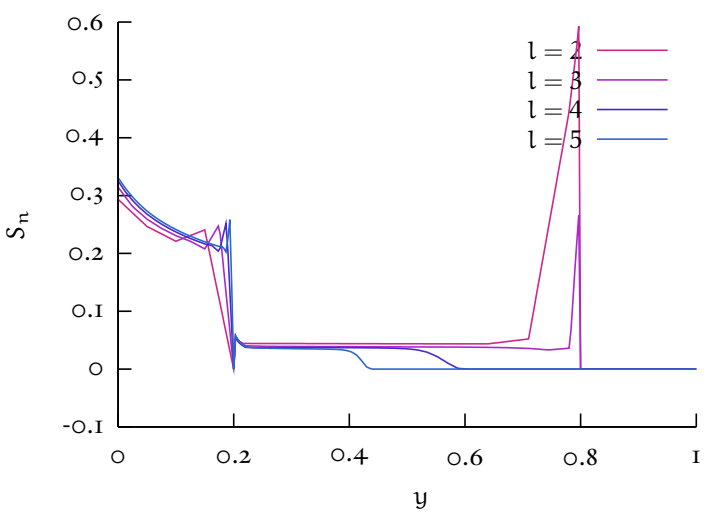

Figure 5.5.: Grid convergence of vertical gas-water flow test case for $t=74 \mathrm{~s}$. The gas saturation along the line in $y$-direction is plotted for grid level $l$ and the fracture widths $b=0.001$ (left) and $b=0.01$ (right). Results in the first row are for the onedimensional fracture geometry, the middle row for the two-dimensional fracture geometry with rectangular ends and the last row for the two-dimensional fracture geometry with triangular ends. 


\section{I. VERTICAL WATER-GAS FLOW}
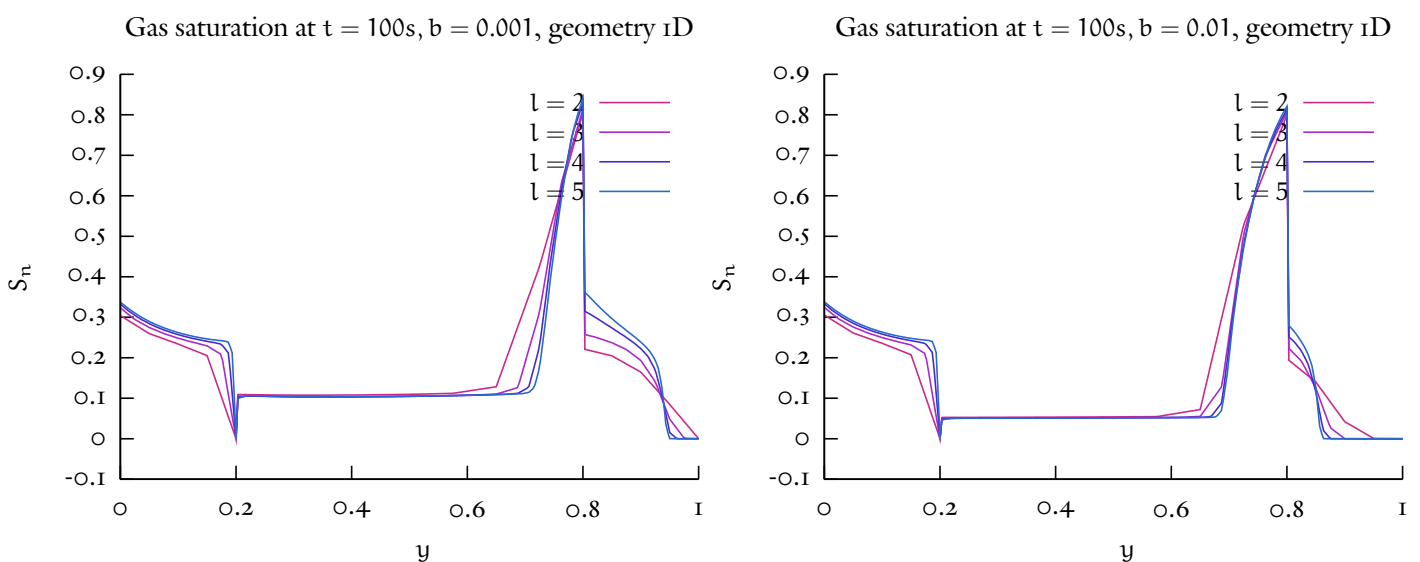

Gas saturation at $\mathrm{t}=100 \mathrm{~s}, \mathrm{~b}=0.001$, geometry $2 \mathrm{D}$ Gas saturation at $\mathrm{t}=100 \mathrm{~s}, \mathrm{~b}=0.01$, geometry $2 \mathrm{D}$
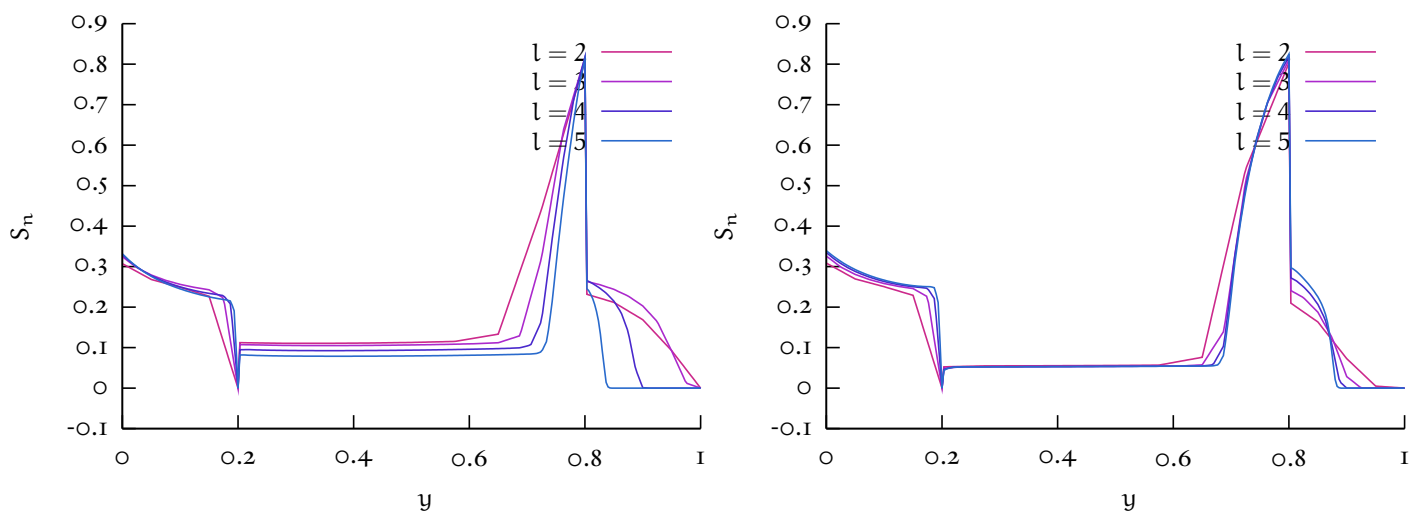

Gas saturation at $\mathrm{t}=100 \mathrm{~s}, \mathrm{~b}=0.001$, geometry $2 \mathrm{DT}$

Gas saturation at $\mathrm{t}=100 \mathrm{~s}, \mathrm{~b}=0.01$, geometry $2 \mathrm{DT}$
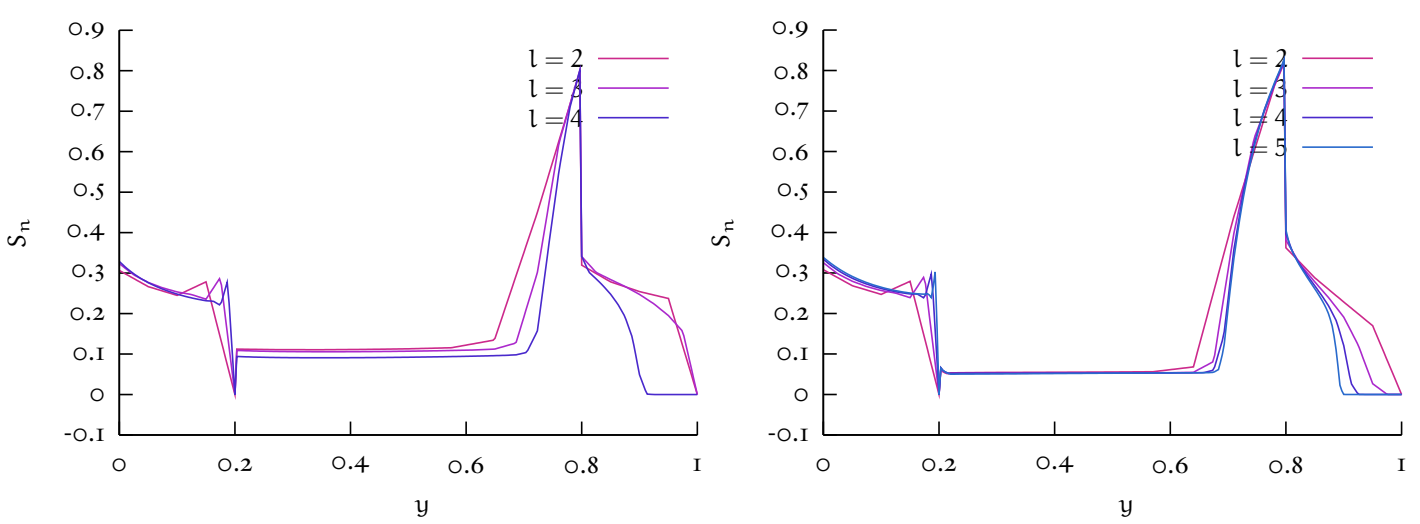

Figure 5.6.: Grid convergence of vertical gas-water flow test case for $t=100 \mathrm{~s}$. The gas saturation along the line in $y$-direction is plotted for grid level $l$ and the fracture widths $b=0.001$ (left) and $b=0.01$ (right). Results in the first row are for the one-dimensional fracture geometry, the middle row for the twodimensional fracture geometry with rectangular ends and the last row for the two-dimensional fracture geometry with triangular ends. 


\section{NumericAl EXPERIMENTS}
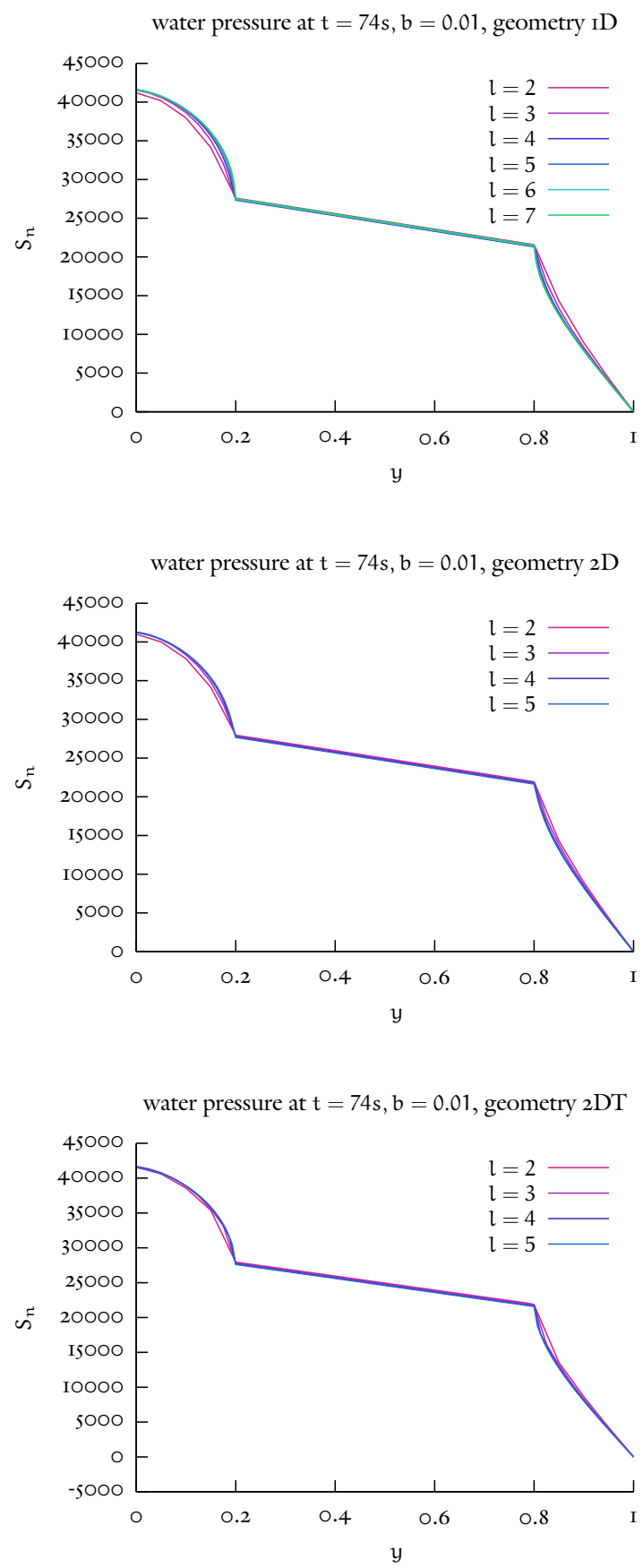

Figure 5.7.: Grid convergence of vertical gas-water flow test case for $t=74 \mathrm{~s}$. The water pressure (labeling of the $y$-axis is wrong) along the line in $y$-direction is plotted for grid level $l$ and the fracture width $b=0.01$. Results at the top are for the one-dimensional fracture geometry, in the middle for the two-dimensional fracture geometry with rectangular ends and the bottom for the two-dimensional fracture geometry with triangular ends. 


\section{I. VERTICAL WATER-GAS FLOW}
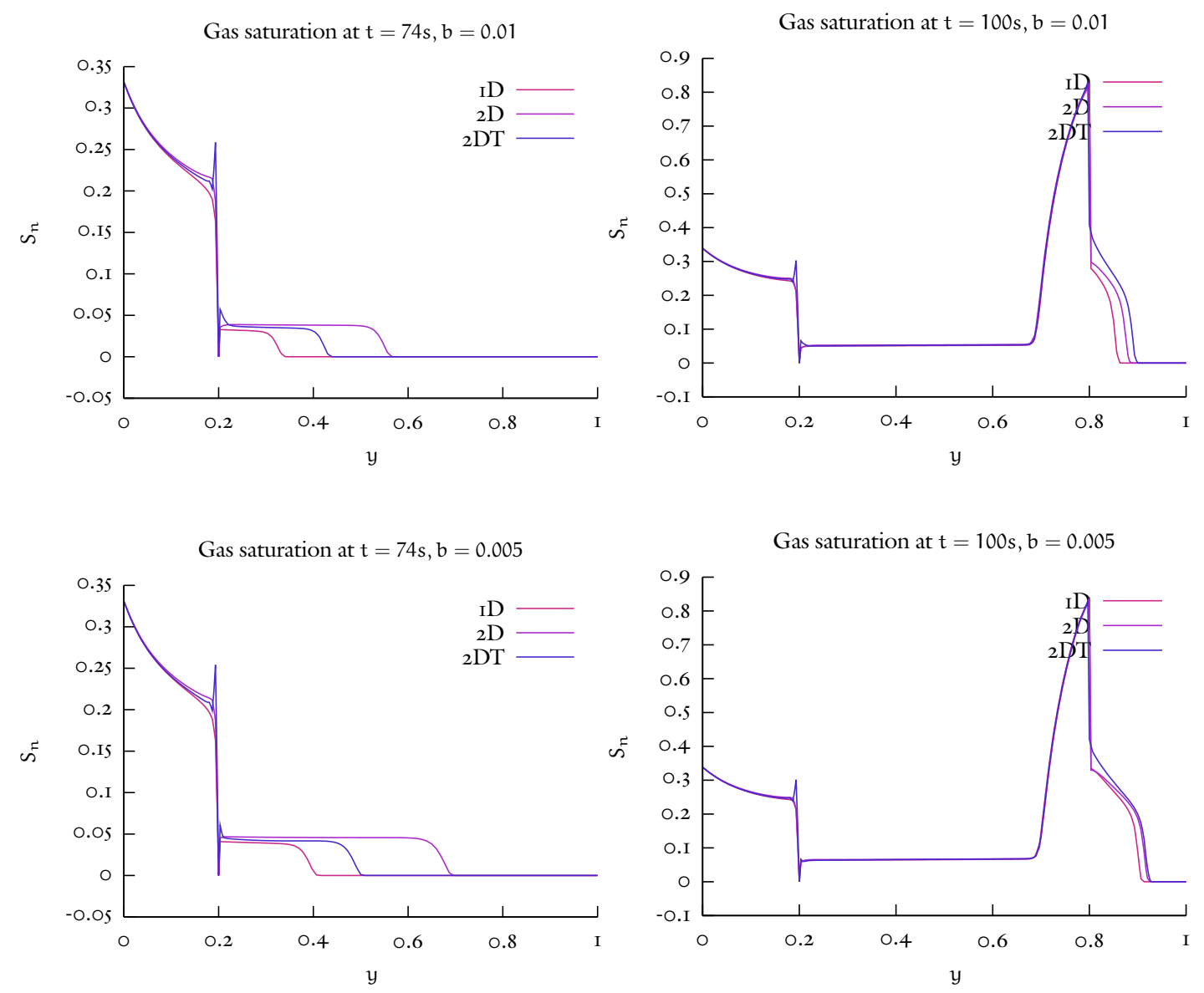

Figure 5.8.: Comparison of the gas saturation value along the vertical axis of the domain on grid level 3. Results in the first row and second row are for fracture widths $b=0.01, b=0.005$ resp., with $t=74 \mathrm{~s}$ in the left column, $t=100 \mathrm{~s}$ in the right column. 


\section{NumericAl EXPERIMENTS}

\subsection{WELL SIMULATION}

The numerical treatment of wells in porous media is a problem of large practical interest in petroleum reservoir engineering and groundwater remediation. The difficulty in well simulation is that a straightforward approach with a line source/sink term fails in heterogeneous porous media, because the line source will act with equal strength on all adjacent grid cells, but should instead take into account the varying permeability. The traditional approach for this problem in a physically correct way is by an integral condition along the well (Peaceman, 1978), which is difficult to implement.

An alternative approach can be taken by employing lower-dimensional elements as presented in the previous section. Assuming that the radius of a borehole is much smaller than its length and the other length scales in the simulation (which is especially true for reservoir simulation with typical length scales on the range of kilometers), a well can be represented as a one-dimensional line. The permeability of the well is much larger than the surrounding well, thereby fulfilling a fundamental requirement of the fracture-matrix flow model.

In figure 5.9 results from a numerical well simulation for a single phase flow problem (2.8) are presented. The domain is $40 \times 10[\mathrm{~m}]$ large, with a pumping well from $(30,2.5)$ to $(30,10) .0 .05[\mathrm{~kg} / \mathrm{s}]$ are pumped from the well. In the case of the lower-dimensional element approach, this is imposed by a Neumann boundary condition at the outflow location of the well-the mass conserving nature of the finite volume method will then be "distributed" along the fracture. In contrast, the line sink approach defines a line along which the size of the sub control volumes of the secondary grid is calculated and then employed in the calculation of the sink term.

For the simulation we used a permeability of $10^{-5}$ in the well and a generated permeability distribution with $\mathrm{K}$-values between $4.61 \cdot 10^{-10}$ and $2.85 \cdot 10^{-9} \cdot \rho$ is chosen as 1000.0 and the $\mu=1$.

Boundary conditions are chosen for both cases as

$$
\begin{aligned}
\rho \mathbf{u} \cdot \mathfrak{n} & =0 & & \text { on }[0,40] \times 0 \\
\rho \mathbf{u} \cdot \mathfrak{n} & =0 & & \text { on }[0,30) \times 10 \\
\rho \mathbf{u} \cdot \mathfrak{n} & =0 & & \text { on }(30,40] \times 10 \\
p(x, y) & =(10-y) * 9810+100000[\mathrm{~Pa}] & & \text { on } 0 \times[0,10] \\
p(x, y) & =(10-y) * 9810+99600[\mathrm{~Pa}] & & \text { on } 40 \times[0,10],
\end{aligned}
$$

In the case of the lower-dimensional element approach we choose

$$
\rho \mathbf{u} \cdot \mathbf{n}=-0.05[\mathrm{~kg} / \mathrm{s}] \quad \text { on } 30 \times 10
$$

on the north boundary, in the case of the line sink we impose the pumping condition through the sink term q.

Figure 5.9 displays the differences in the models. While the pressure in the well is independent of the surrounding matrix permeability when using the fracture, the line sink exhibits a 

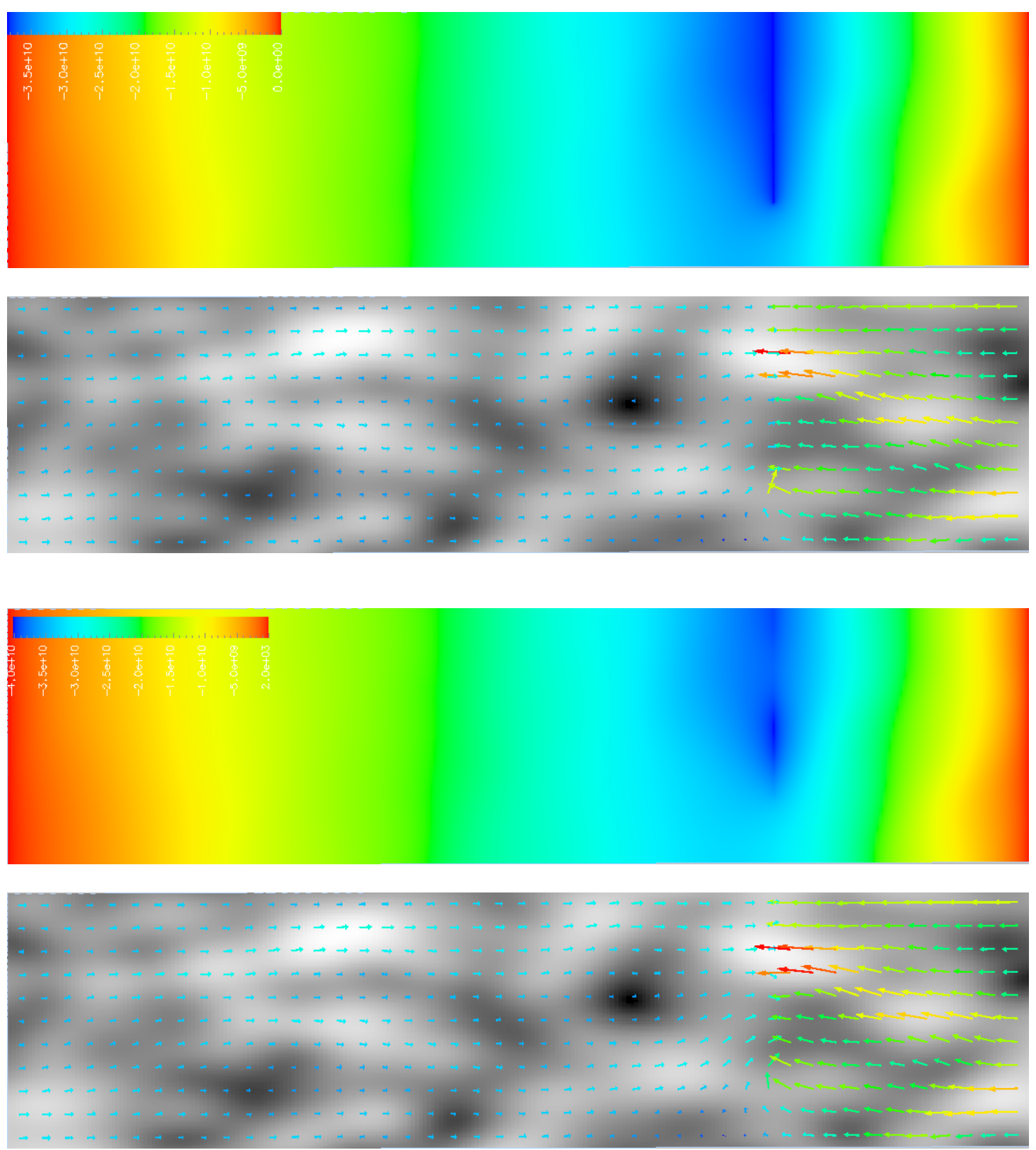

Figure 5.9.: Numerical simulation of a pumping well. From top to bottom: (I) The pressure field for the simulation with a fracture. (2) The velocity distribution and the Permeability field for the fracture simulation. (I) The pressure field for the simulation with a line sink. (2) The velocity distribution and the Permeability field for the line sink simulation.

variation in the pressure induced by the permeability. In the darker regions, where the permeability is smaller, the pressure is larger in the case of the line sink, because larger suction 


\section{Numerical EXPERIMENTS}

is necessary to draw the water from the medium.

The computations are done on a grid with 32768 quadrilateral elements and 33153 nodes, running on a $800 \mathrm{MHz}$ PowerBook $\mathrm{G}_{4}$.

\subsection{Three-Dimensional Oil-Water simulation}

The next numerical experiment simulates the flow of an incompressible dense oil phase through a network. Although we are mainly interested in gas-water flow, we include this experiment to show the flexibility of the method.

The enclosing domain has a size of $12 \times 12 \times 18[\mathrm{~m}]$ and contains an interconnected fracture network with eight fractures. Two fractures are connected to the domain boundary, it is through these fractures that oil enters the system. This simulates a contamination of a soil with dense oil.

The parameters of the simulation are

$$
\begin{aligned}
\rho_{w} & =1000\left[\mathrm{~kg} / \mathrm{m}^{3}\right] & \rho_{\mathrm{n}} & =1560\left[\mathrm{~kg} / \mathrm{m}^{3}\right] \\
\mu_{w} & =10^{-3}[\mathrm{~Pa} \mathrm{~s}] & \mu_{\mathrm{n}} & =1.2 \cdot 10^{-3}[\mathrm{~Pa} \mathrm{~s}] \\
\Phi^{f} & =0.3 & \Phi^{\mathrm{m}} & =0.1 \\
\mathrm{~K}^{f} & =10^{-8} & \mathrm{~K}^{\mathrm{m}} & =10^{-12} \\
S_{w r}^{f} & =0 & S_{w r}^{m} & =0 \\
S_{\mathrm{gr}}^{f} & =0 & S_{\mathrm{gr}}^{m} & =0 \\
\lambda^{f} & =2 & \lambda^{\mathrm{m}} & =2 \\
\mathrm{p}_{\mathrm{d}}^{\mathrm{f}} & =2432.39[\mathrm{~Pa}] & \mathrm{p}_{\mathrm{d}}^{\mathrm{m}} & =5439.0[\mathrm{~Pa}]
\end{aligned}
$$

We prescribe a hydrostatic pressure at the boundaries, $p_{w}=9810+z * 9810$ (with $z$ directed downwards). The boundary condition for the saturation is $S n=0$, except for the lines where the topmost two fractures intersect the domain boundary. Here we use $S n=0.4$.

The number of elements and nodes in the grid for uniform refinement and adaptive refinement (from level 1) is shown in the following table.

\begin{tabular}{lrrrr}
\hline & \multicolumn{2}{c}{ uniform refinement } & \multicolumn{2}{c}{ adaptive refinement } \\
Level l & \#E & $\# N$ & $\# \mathrm{E}$ & $\# \mathrm{~N}$ \\
\hline $\mathrm{O}$ & $\mathrm{II} 43$ & & $\mathrm{II} 43$ & 300 \\
$\mathrm{I}$ & $9 \mathrm{I} 44$ & & $9 \mathrm{I} 44$ & $\mathrm{I} 943$ \\
2 & $73 \mathrm{I} 52$ & & 52782 & 9584 \\
3 & $5852 \mathrm{I} 6$ & & 224747 & $404 \mathrm{I3}$ \\
4 & $468 \mathrm{I} 728$ & & 848863 & $\mathrm{I} 52319$ \\
5 & 37453824 & 6345473 & 3199899 & 573632 \\
\hline
\end{tabular}

We ran the simulation in two configurations, one parallel computation with uniform refinement and a sequential computation with adaptive refinement. Due to the adaptive refinement we were able to achieve the same level of refinement in the fractures for both cases. 

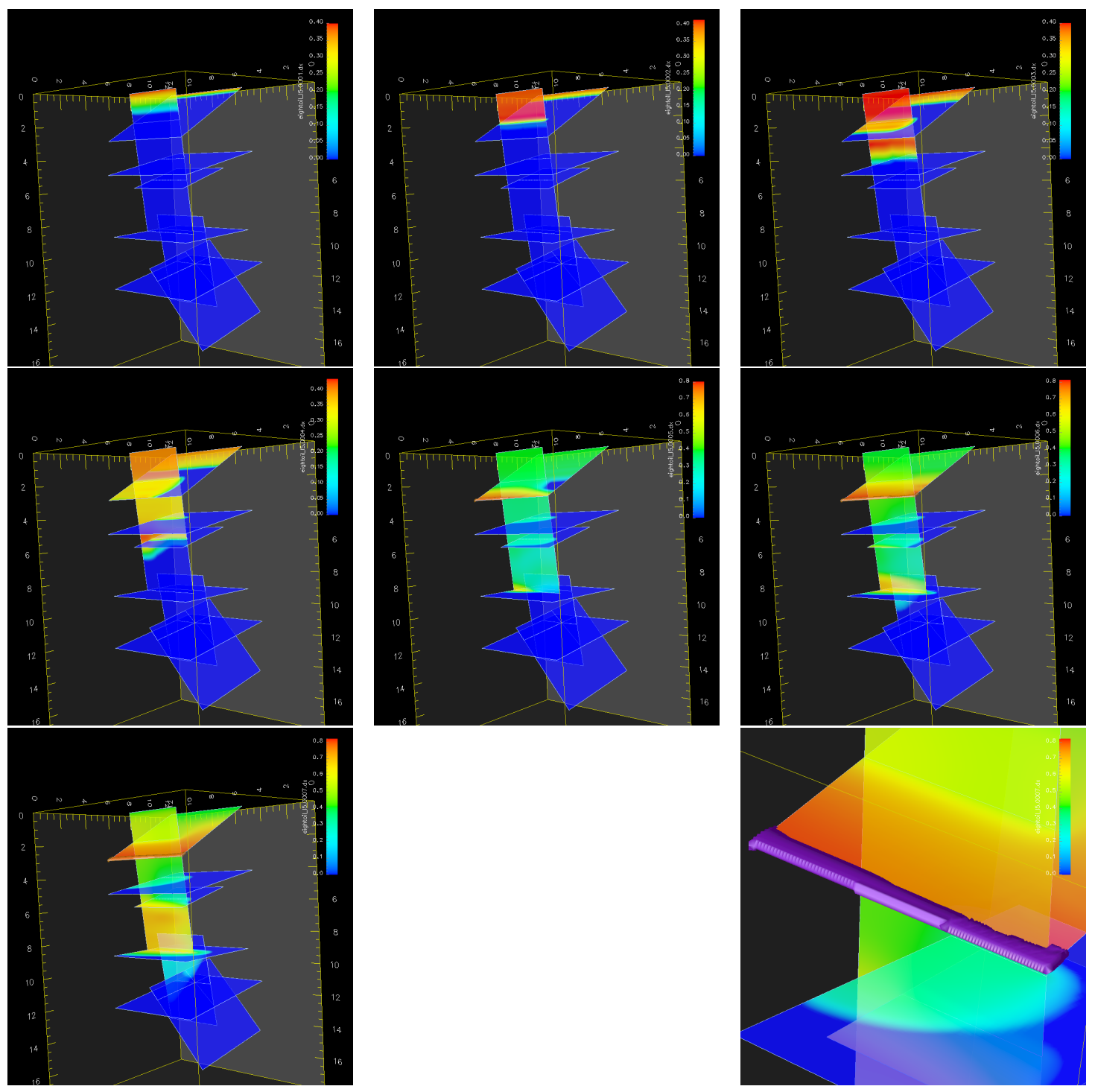

Figure 5.IO.: $3 \mathrm{D}$ Water-Oil simulation

The adaptive refinement criterion for the elements was based on the neighborhood to a fracture and the resulting meshes are shown in figure 3.7 . The sequential results were obtained on a I.8 GHz PowerMac G5 with 4 GB DDR SDRAM main memory. Parallel computations were performed on the HELICS cluster (HEidelberg LInux Cluster System) consisting of AMD Athlon I.3 GHz processors connected by a Myrinet 2000 interconnect.

Figure 5.IO shows visualizations for seven time steps from the sequential computation and a close-up of a detail from the last time step. It is visible that the expected physical behavior is achieved by the numerical simulation. Oil flows at different velocities through the fractures, depending on the angle of inclination of the fractures. The fastest movement 


\section{Numerical EXPERIMENTS}

can be observed in the vertical fractures. At fracture intersections a spreading of the fluid is visible, with preferred fluid flow in the vertical direction. At the fracture ends the oil is pooled. With the chosen parameters, the rock matrix is penetrated for $S_{n}=0.8$. This saturation is reached in the fifth time step. The detail in figure 5.IO shows the isosurface for the saturation $S_{m}=0.01$ in the matrix. Oil has penetrated the matrix along the whole fracture end line, but movement in the matrix is only very slow.

The simulation uses the finite volume scheme with full upwinding and the fractionalstep- $\theta$ scheme. In each Newton step a defect reduction of $10^{-5}$ is prescribed, and a linear defect reduction of $10^{-5}$. The multigrid method uses a $\mathrm{V}(2,2)$-cycle with symmetric GaußSeidel smoothing. The smoothing is damped with a factor 0.8. The time step size changes depending on the number of required nonlinear iterations.

The next table shows the time steps for the sequential program, the total number of linear multigrid iterations, the number of linear multigrid iterations in the time step, number of nonlinear Newton iterations, and the number of nonlinear Newton iterations in the time step.

\begin{tabular}{lrrrrr}
\hline $\mathrm{T}$ & $\Delta \mathrm{t}$ & \# lin & lin/\#t & \# nonlin & nonlin/\#t \\
\hline 0.125 & 0.125 & $\mathrm{I} 09$ & $\mathrm{I} 09$ & $\mathrm{I} 9$ & $\mathrm{I} 9$ \\
0.375 & 0.25 & 406 & 297 & 46 & 27 \\
0.875 & 0.5 & 992 & 586 & 92 & 46 \\
$\mathrm{I} .375$ & 0.5 & $\mathrm{I} 626$ & 634 & $\mathrm{I} 4 \mathrm{I}$ & 49 \\
2.375 & $\mathrm{I}$ & 2788 & $\mathrm{II} 62$ & 224 & 83 \\
3.375 & $\mathrm{I}$ & 4054 & $\mathrm{I} 266$ & 274 & 50 \\
\hline
\end{tabular}

Note that one time step consists of three substeps. The convergence rate of the multigrid method is $\approx 0.2$. When ILU is employed the convergence rate is better. However, it is the convergence of the nonlinear solver which-if improved-would greatly enhance the performance of the program.

The analogous results for the adaptive computation on level 3 are listed below. Here we see a solution behavior that is typical: The number of iterations in the time steps increases until $\mathrm{T} \approx 20$ s. After this point, the matrix has been infiltrated and the influence of the steep gradient of the capillary pressure function decreases.

\begin{tabular}{lrrrrr}
\hline $\mathrm{T}$ & $\Delta \mathrm{t}$ & \# lin & $\operatorname{lin} / \Delta \mathrm{t}$ & \# nonlin & nonlin/ $\Delta \mathrm{t}$ \\
\hline $0 . \mathrm{I} 25$ & $0 . \mathrm{I} 25$ & $6 \mathrm{I}$ & $6 \mathrm{I}$ & $\mathrm{I} 3$ & $\mathrm{I} 3$ \\
0.375 & 0.25 & $\mathrm{I} 63$ & $\mathrm{IO} 2$ & 26 & $\mathrm{I} 3$ \\
0.875 & 0.5 & 339 & $\mathrm{I} 76$ & 45 & $\mathrm{I} 9$ \\
$\mathrm{I} .875$ & $\mathrm{I}$ & 588 & 249 & $7 \mathrm{I}$ & 26 \\
3.875 & 2 & $\mathrm{IO} 0$ & 482 & $\mathrm{IIO}$ & 39 \\
7.875 & 4 & $2 \mathrm{II} 5$ & $\mathrm{IO} 45$ & $\mathrm{I} 75$ & 65 \\
$\mathrm{I5.875}$ & 8 & $4 \mathrm{OII}$ & $\mathrm{I} 896$ & 262 & 87 \\
20 & 4.125 & $53 \mathrm{I} 4$ & $\mathrm{I} 303$ & 333 & $7 \mathrm{I}$ \\
24 & 4 & 6526 & $\mathrm{I} 2 \mathrm{I2}$ & 390 & 57 \\
32 & 8 & 7666 & $\mathrm{II} 4 \mathrm{O}$ & 434 & 44 \\
\hline
\end{tabular}


The large number of Newton iterations stems from the large Peclet numbers which arise in the current configuration. We assume that the Peclet number is approximately 10, which proves the robustness of the algorithm. It is also clear that for accuracy reasons smaller time steps should be employed. Note also that the fractional-step- $\theta$ scheme has three sub steps.

\subsection{Three-Dimensional Gas-Water simulation}

The enclosing domain for the gas-water flow simulation has a size of $12 \times 12 \times 18$ [m] and contains an interconnected fracture network with eight fractures. Two fractures are connected to the domain boundary, it is through these fractures that the compressible gas phase enters the system.

The parameters of the simulation are

$$
\begin{aligned}
\rho_{w} & =1000\left[\mathrm{~kg} / \mathrm{m}^{3}\right] & \rho_{\mathrm{g}} & =\mathrm{p}_{\mathrm{n}} / 84149.6\left[\mathrm{~kg} / \mathrm{m}^{3}\right] \\
\mu_{w} & =10^{-3}[\mathrm{~Pa} \mathrm{~s}] & \mu_{\mathrm{g}} & =1.65 \cdot 10^{-5}[\mathrm{~Pa} \mathrm{~s}] \\
\Phi^{f} & =0.3 & \Phi^{\mathrm{m}} & =0.1 \\
\mathrm{~K}^{f} & =10^{-8} & \mathrm{~K}^{\mathrm{m}} & =10^{-12} \\
S_{w r}^{f} & =0 & S_{w r}^{m} & =0 \\
S_{g r}^{f} & =0 & S_{g r}^{m} & =0 \\
\lambda^{f} & =2 & \lambda^{\mathrm{m}} & =2 \\
p_{\mathrm{d}}^{f} & =1000[\mathrm{~Pa}] & \mathrm{p}_{\mathrm{d}}^{\mathrm{m}} & =2000[\mathrm{~Pa}]
\end{aligned}
$$

We prescribe a hydrostatic pressure at the boundaries with $p_{w}=9810$ at the north boundary. The boundary condition for the saturation is $S n=0$, except for the south boundary with Neumann boundary conditions $\phi=0$ except for the lines where the fractures intersect the domain boundary. There we set $\phi=-2.4 \cdot 10^{-4}$.

Figure 5.II shows the time step size development for the problem. Again, the Courant number is very large and makes the system difficult to solve. The fractional-step- $\theta$ scheme has three sub steps, so the numbers have to be divided by three for comparison with other time stepping schemes.

The simulation uses the finite volume scheme with full upwinding and the fractionalstep- $\theta$ scheme. In each Newton step a defect reduction of $10^{-5}$ is prescribed, and a linear defect reduction of $10^{-5}$. The multigrid method uses a $\mathrm{V}(2,2)$-cycle with symmetric GaußSeidel smoothing. The smoothing is damped with a factor 0.8. The time step size changes depending on the number of required nonlinear iterations.

In figure 5.12 we show visualizations for the time steps $t=40 \mathrm{~s}, t=60 \mathrm{~s}, t=80 \mathrm{~s}$. The gas saturation is small in these examples. The large picture shows the gas saturation for $t=80 \mathrm{~s}$. Contrary to the Water-Oil problem the gas spreads into a very thin layer along the fracture end which is hardly visible. This is due to the smaller amount of mass that has entered the system and due to the substantial viscosity and density differences between gas and water. 


\section{Numerical experiments}
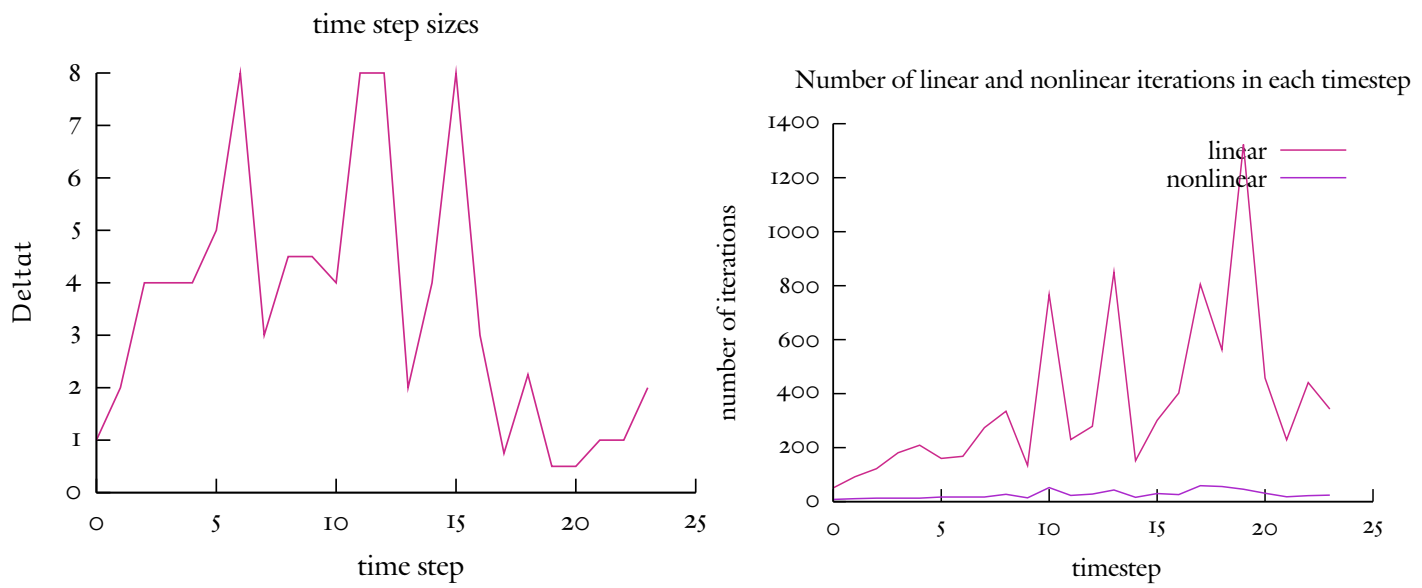

Figure 5.II.: $3 \mathrm{D}$ Water-Gas simulation 
5.4. Three-Dimensional Gas-Water simulation
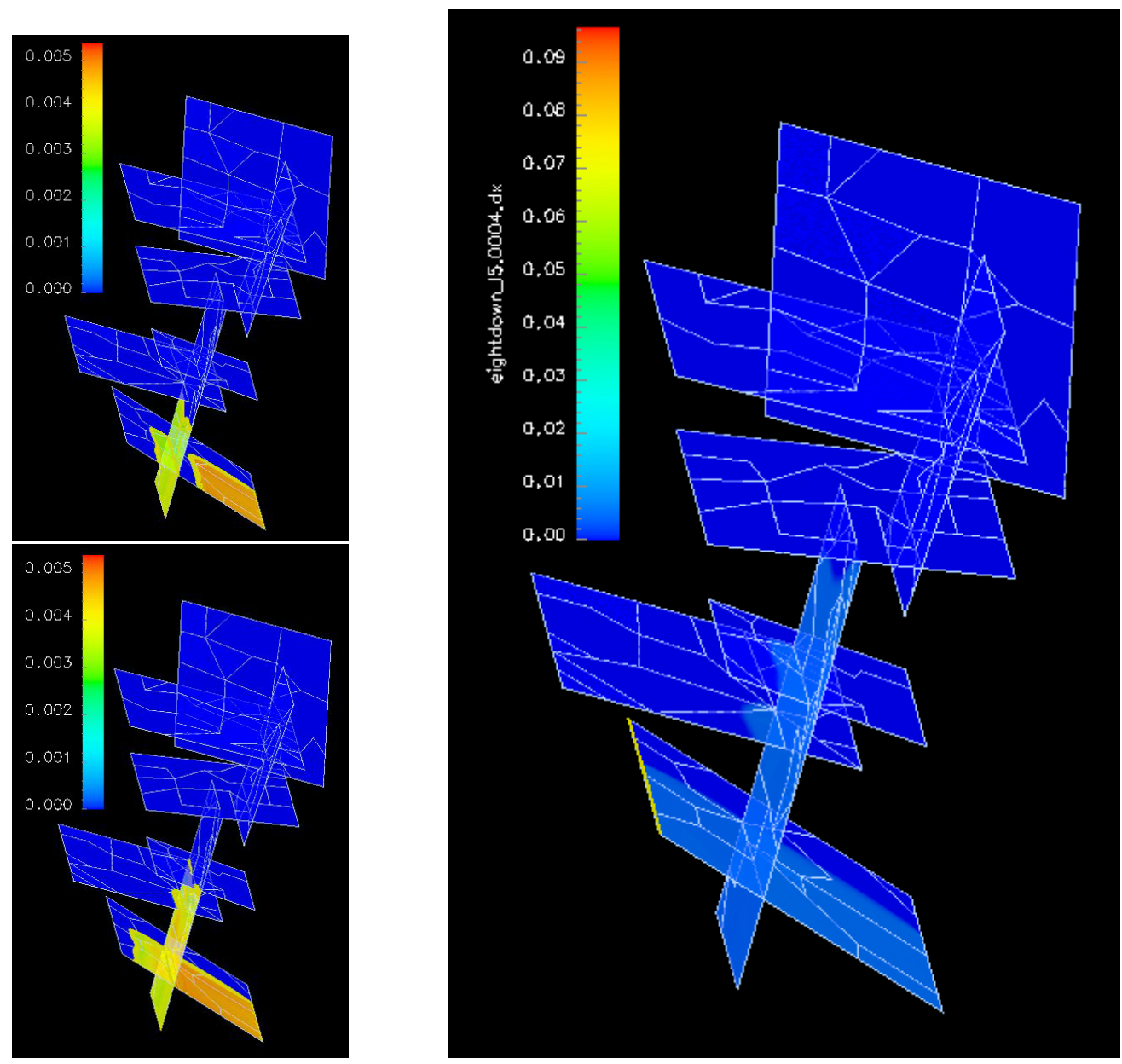

Figure 5.I2.: $3 \mathrm{D}$ Water-Gas simulation at $\mathrm{t}=40 \mathrm{~s}$ (upper left), $\mathrm{t}=60 \mathrm{~s}$ (lower left), $\mathrm{t}=80 \mathrm{~s}$ (right). 
5. NUMERICAL EXPERIMENTS 


\section{Conclusion}

We have demonstrated an interdisciplinary approach to the numerical simulation of multiphase flow in fractured porous media. Recognizing the importance of the interaction between the components of the simulation process, from the physical model over the mathematical and numerical model to the computer program, we presented a balanced view of the problem.

Among the competing models for fluid flow and transport in fractured media, we chose the discrete modeling approach in which discrete fractures are modeled separately, assuming a continuum in the fractures and the surrounding porous media. This has the advantage over equivalence models that no artificial parameters-like exchange terms between fracture and matrix in the double porosity model-have to be employed. We assumed the existence of a representative elementary volume (REV) in the matrix and the fracture, from which we can derive a multiphase flow extension of Darcy's law, and assume that capillary pressure functions and relative permeability functions according to Brooks-Corey are valid in the fractures. The behavior at the fracture-matrix interface and general media discontinuities is modeled by the extended capillary pressure conditions of van Duijn et al. (I995). The large role of the capillary pressure has long been recognized and is an important component of the model.

For the numerical simulation two schemes were considered, a vertex centered finite volume scheme and the discontinuous Galerkin method of Baumann and Oden. The latter is in a less mature state and has not seen applications to multiphase fluid flow in heterogeneous media yet. We applied the method to fluid flow of a single phase in heterogeneous media and presented a multigrid method for the efficient solution of the groundwater equation. The multigrid method shows $h$-independent convergence for homogeneous and heterogeneous permeability distributions and performs dramatically better than a finite volume scheme in the case of problems with full regularity. The cache-efficient nature of the small dense blocks arising in the discontinuous Galerkin method makes it especially suited for modern computer architectures. We also compared the discontinuous Galerkin method to the vertex centered finite volume scheme in the case of a problem with a single fracture. In this example we also considered the influence of fracture geometries on the solution, which is an issue if fractures are modeled as volumetric elements. Depending on the shape of the element at the fracture ends, the velocity field changes considerably between the different realizations. We also found good agreement between a finite volume method with lowerdimensional fracture elements, the volumetric finite volume method and the discontinuous Galerkin method in the case of quadrilateral fracture end.

For the two-phase flow equation we presented a method based on a phase pressuresaturation formulation. The method treats the two-phase fracture-matrix-system in a fully coupled way in order to capture the effects of fracture-matrix interaction adequately. The 


\section{Conclusion}

method is based on a vertex centered finite volume scheme which implements fracture elements of lower dimension. Due to the incorporation of the extended capillary pressure function it is possible to achieve the physically relevant discontinuous saturation at nodes shared by fracture and matrix in a conforming finite element setting. The discretized nonlinear equations are solved by an inexact Newton method with line search, the linearized equations are solved by a parallel multigrid method with special truncated restriction operators. The implementation of the computer program is based on the framework UG. Multiphase flow problems in porous media are ideally suited for adaptive refinement; their complex geometries require unstructured grid methods and the complexity of the problems requires to run computations on parallel computers. We were able to demonstrate that the method can be employed for simulations in complex fracture-matrix systems with several million unknowns. The simulation software was embedded in an environment of domain generators, grid generators and visualization, which had to be adapted for the lower-dimensional element representation.

The methodology presented in this work can serve as a starting point for future developments. For crystalline rocks it would be very attractive to go beyond the convectiondiffusion model and to extend the model to multi-component flow, which would allow to include the long-term behavior of the system and would provide an efficient tool for longterm safety assessment. On longer time scales degassing becomes an important issue. The inclusion of heterogeneous permeabilities is also possible for the simulator and is already implemented. Experimental data is sparse or non-existent for these problems, but stochastically generated permeability fields offer an alternative with which simulation experiments to investigate channeling in fracture networks are possible. 


\section{A. The Hele-Shaw Analog for Viscous Flow in Parallel Plates}

In this section we explain how the cubic law for fluid flow in a single fracture from section 2.2.2 can be derived from the Navier-Stokes equations. This analog is called the HeleShaw analog or parallel plate model. We follow the description in Bear (I993).

We consider the Navier-Stokes equations

$$
\rho \frac{\partial \boldsymbol{v}}{\partial \mathrm{t}}-\mu \Delta \boldsymbol{v}+\rho \nabla \cdot\left(\boldsymbol{v} \boldsymbol{v}^{\top}\right)+\nabla p-\rho \mathbf{g}=0
$$

where $\rho$ is the density of the fluid, $\mu$ is the dynamic viscosity, $p$ is the pressure, $v$ is the fluid's velocity, $t$ is time, and $\mathbf{g}$ is the vector of gravitational acceleration. We assume that the fracture can be described by a fracture plane and a fracture width associated with every point of the fracture plane, such that the width is much smaller than any length of interest in the other directions. We then introduce an orthogonal coordinate system $(\xi, \eta, \zeta)$, where $\xi$ and $\eta$ in the axis' plane (see figure A). The stationary fracture walls can the be described by

$$
F_{1}(\xi, \eta, \zeta)=\zeta_{1}-f_{1}(\xi, \eta)=0, \quad F_{2}(\xi, \eta, \zeta)=\zeta_{2}-f_{2}(\xi, \eta)=0,
$$

where $\zeta$ is the coordinate normal to the fracture plane, and $\zeta_{1}$ and $\zeta_{2}$ are the values of $\zeta$ on the fracture wall. The outward normal unit vector at a point on the fracture wall is

$$
\mathfrak{n}_{\mathrm{i}}=\frac{\nabla \mathrm{F}_{\mathrm{i}}}{\left|\nabla \mathrm{F}_{\mathrm{i}}\right|}, \quad \mathrm{i}=1,2,
$$

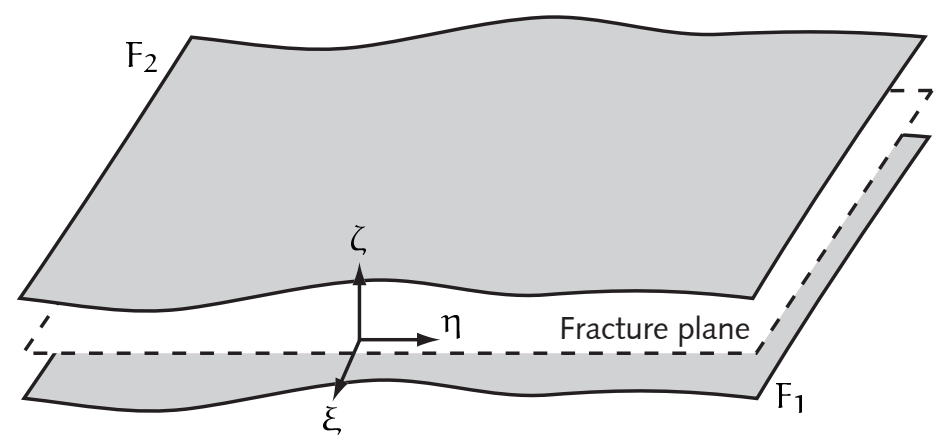

Figure A.I.: Nomenclature for the parallel plate model. 


\section{A. The Hele-Shaw Analog for Viscous Flow in Parallel Plates}

and the fracture width is

$$
b(\xi, \eta)=f_{2}(\xi, \eta)-f_{1}(\xi, \eta)
$$

To derive an averaged equation, we integrate (A.I) across the fracture width normal to the fracture axis and additionally employ the piezometric head $h$ instead of the pressure $p$

$$
\mathrm{h}=\frac{\mathrm{p}}{\rho g}+z
$$

and arrive at

$$
\int_{f_{1}}^{f_{2}}\left(\rho \frac{\partial v}{\partial t}-\mu \Delta \boldsymbol{v}+\rho \nabla \cdot\left(\boldsymbol{v} \boldsymbol{v}^{\top}\right)+\nabla h-\rho \mathbf{g}\right) \mathrm{d} \zeta=0
$$

Now we can investigate the possible simplifications in each term of (A.6), starting by deriving the average momentum balance equation in the fracture plane. To this end we introduce for quantities averaged across the fracture width the notation

$$
\widetilde{g}=\frac{1}{b} \int_{f_{1}}^{f_{2}} g(\xi, \eta, \zeta) d \zeta .
$$

The velocity is split into the velocity vector in the fracture plane and a velocity deviation $\grave{v}$, such that

$$
\boldsymbol{v}(\xi, \eta, \zeta)=\widetilde{\boldsymbol{v}}(\xi, \eta)+\grave{v}(\xi, \eta, \zeta), \quad \widetilde{\boldsymbol{v}}=0 .
$$

For the velocity product $v v^{\top}$ the relation

$$
\widetilde{v v^{\top}}=\widetilde{v} \widetilde{v^{\top}}+\widetilde{v} \hat{v}^{\top}
$$

holds, where $\widetilde{\boldsymbol{v} \hat{v}^{\top}}$ represents the dispersive momentum flux.

The first term in $($ A.6 $)$ is then

$$
\int_{f_{1}}^{f_{2}} \rho \frac{\partial v}{\partial t} d \zeta=\rho \frac{\partial v}{\partial t}\left(\int_{f_{1}}^{f_{2}} v d \zeta\right)=\rho \frac{\partial(b \widetilde{v})}{\partial t} .
$$

The second term in (A.6) evaluates to

$$
\begin{aligned}
& \int_{f_{1}}^{f_{2}} \rho \nabla \cdot\left(\boldsymbol{v} \boldsymbol{v}^{\top}\right) \mathrm{d} \zeta=\rho \nabla_{\xi, \eta} \cdot \int_{f_{1}}^{f_{2}} \boldsymbol{v} \boldsymbol{v}^{\top} \mathrm{d} \zeta-\left.\rho \boldsymbol{v} \boldsymbol{v}^{\top}\right|_{f_{2}} \cdot \nabla \mathrm{F}_{2}+\left.\rho \boldsymbol{v} \boldsymbol{v}^{\top}\right|_{f_{1}} \cdot \nabla \mathrm{F}_{1} \\
& =\rho \nabla_{\xi, \eta} \cdot\left(\widetilde{b v v^{\top}}\right)-\left.\rho v v^{\top}\right|_{f_{2}} \cdot \nabla F_{2}+\left.\rho v v^{\top}\right|_{f_{1}} \cdot \nabla F_{1} \\
& =\rho \nabla_{\xi, \eta} \cdot\left(\boldsymbol{b} \widetilde{\boldsymbol{v}} \widetilde{\boldsymbol{v}}^{\top}\right)+\rho \nabla_{\xi, \eta} \cdot(\boldsymbol{b} \widetilde{\boldsymbol{v} \hat{v}})-\left.\rho \boldsymbol{v} \boldsymbol{v}\right|_{f_{2}} \cdot \nabla F_{2}+\left.\rho \boldsymbol{v} \boldsymbol{v}\right|_{f_{1}} \cdot \nabla F_{1}
\end{aligned}
$$


where $\nabla_{\xi, \eta}$ denotes differentiation with respect to the fracture plane coordinates $(\xi, \eta)$ only.

The third term of A.6 is

$$
\int_{f_{1}}^{f_{2}} \rho g \nabla h d \zeta=\rho g \nabla_{\xi, \eta}(b \tilde{h})-\left.\rho g h\right|_{f_{2}} \nabla F_{2}+\left.\rho g h\right|_{f_{1}} \nabla F_{1}
$$

and the fourth term

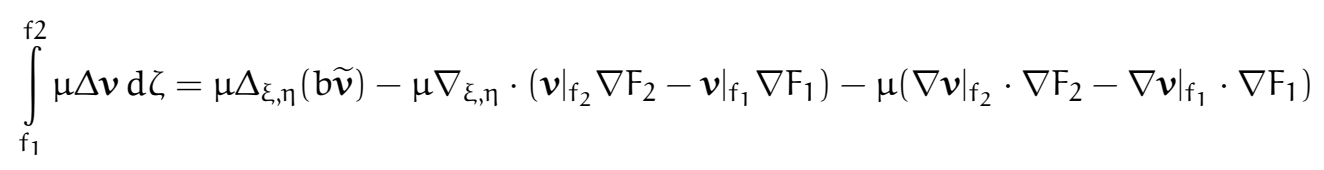

Altogether, this is the averaged linear momentum balance equation in the fracture plane:

$$
\begin{array}{r}
\rho \frac{\partial(\mathrm{b} \widetilde{\boldsymbol{v}})}{\partial \mathrm{t}}+ \\
\rho \nabla_{\xi, \eta} \cdot\left(\mathrm{b} \widetilde{\boldsymbol{v}} \widetilde{\boldsymbol{v}}^{\mathrm{T}}\right)+\rho \nabla_{\xi, \eta} \cdot(\mathrm{b} \widetilde{\boldsymbol{v} \boldsymbol{v}})-\left.\rho \boldsymbol{v} \boldsymbol{v}\right|_{\mathrm{f}_{2}} \cdot \nabla \mathrm{F}_{2}+\left.\rho \boldsymbol{v} \boldsymbol{v}\right|_{\mathrm{f}_{1}} \cdot \nabla \mathrm{F}_{1}+ \\
\rho g \nabla_{\xi, \eta}(\mathrm{b} \widetilde{h})-\left.\rho g h\right|_{\mathrm{f}_{2}} \nabla \mathrm{F}_{2}+\left.\rho g\right|_{\mathrm{f}_{1}} \nabla \mathrm{F}_{1}+ \\
\mu \Delta_{\xi, \eta}(\mathrm{b} \widetilde{\boldsymbol{v}})-\mu \nabla_{\xi, \eta} \cdot\left(\left.\boldsymbol{v}\right|_{\mathrm{f}_{2}} \nabla \mathrm{F}_{2}-\left.\boldsymbol{v}\right|_{\mathrm{f}_{1}} \nabla \mathrm{F}_{1}\right)-\mu\left(\left.\nabla \boldsymbol{v}\right|_{\mathrm{f}_{2}} \cdot \nabla \mathrm{F}_{2}-\left.\nabla \boldsymbol{v}\right|_{\mathrm{f}_{1}} \cdot \nabla \mathrm{F}_{1}\right)=0
\end{array}
$$

and the averaged mass balance equation is

$$
\frac{\partial(\mathrm{b} \widehat{\rho})}{\partial t}+\nabla_{\xi, \eta} \cdot(b \widehat{\rho} \widehat{\boldsymbol{v}})-\left.(\rho \boldsymbol{v})\right|_{f_{2}} \cdot \nabla \mathrm{F}_{2}+\left.(\rho \boldsymbol{v})\right|_{f_{1}} \cdot \nabla \mathrm{F}_{1}=0 .
$$

At this point we can employ the assumptions imposed on the parallel plate model, which are:

$\triangleright$ The fracture walls are stationary parallel plates with an impervious smooth surface.

$\triangleright$ Fluid density is constant.

$\triangleright$ The fluid flow is stationary. Without loss of generality we assume that flux flow is in the direction of the $\xi$-coordinate.

$\triangleright$ The dispersive momentum flux is much smaller than the advective momentum flux, $\left|\widetilde{\hat{v} \hat{v}^{\top}}\right| \ll \widetilde{\boldsymbol{v}} \widetilde{\boldsymbol{v}}^{\top}$.

$\triangleright$ Changes in the piezometric head across apertures are negligible, $\left.\left.h\right|_{f_{1}} \simeq h\right|_{f_{2}}$. (If the fracture walls are not parallel, the condition $\left.\left.h\right|_{f_{1}} \simeq h\right|_{f_{2}} \simeq \widetilde{h}$ is required.)

This reduces $\mathrm{A} .8$ to

$$
\nabla_{\xi, \eta} \cdot(\mathbf{b} \widehat{\rho} \widehat{\boldsymbol{v}})-\left.(\rho \boldsymbol{v})\right|_{f_{2}} \cdot \nabla \mathrm{F}_{2}+\left.(\rho \boldsymbol{v})\right|_{f_{1}} \cdot \nabla \mathrm{F}_{1}=0 .
$$

Substituting A.9 in A.7 and applying the assumptions yields the equation

$$
\rho g b \frac{d \tilde{h}}{d \xi}-\mu\left(\left.\frac{\partial v_{\xi}}{\partial \zeta}\right|_{f_{2}}-\left.\frac{\partial v_{\xi}}{\partial \zeta}\right|_{f_{1}}\right)=0
$$


where $v_{\xi}$ is the $\xi$-component of the velocity vector $\boldsymbol{v}$. If we assume a no-slip boundary condition $v_{\xi}=0$ at the fracture walls and a laminar flow regime, the velocity distribution will be parabolic about the fracture axis,

$$
v_{\xi}(\eta)=\frac{6 \widehat{v}_{\xi}}{b^{2}}\left(\frac{1}{2} b+\eta\right)\left(\frac{1}{2} b-\eta\right) \quad-\frac{1}{2} b \leq \eta \leq \frac{1}{2} b .
$$

Combined with (A.IO) this can be rewritten

$$
\widetilde{v_{\xi}}=-\frac{\rho g}{\mu} \frac{b^{2}}{12} \frac{d \tilde{h}}{d \eta}
$$

which is just 2.I2), expressed with the hydraulic conductivity

$$
k=\frac{\rho g}{\mu} \frac{b^{2}}{12}
$$

instead of the permeability K. The hydraulic conductivity and the permeability are related by

$$
k=-\frac{\rho g}{\mu} K \text {. }
$$

The assumption of smooth fracture walls does not apply in reality and as early as 195I Lomize investigated the influence of fracture roughness. He introduced the concept of roughness $\varepsilon$ defined in terms of the absolute height of the asperities, and found the empirical equation

$$
\Psi=\frac{96}{\operatorname{Re}} \underbrace{\left(1+6.0\left(\frac{\varepsilon}{2 b}\right)^{\frac{3}{2}}\right)}_{=f}
$$

which is valid for $\varepsilon /(2 b)>0.065$. $f=1$ for smooth surfaces and $f>1$ for rough fracture surfaces. Witherspoon et al. (I980) confirmed the validity of the cubic law for fractures with widths between $4 \mu \mathrm{m}$ and $250 \mu \mathrm{m}$, independent of the rock material and with values of $\mathrm{f}$ between 1.04 and 1.65 .

If the fracture wall permeability is not neglected in (A.7) and (A.8), we obtain instead of A.IO the equation

$$
\rho g b \frac{d \widetilde{h}}{d \xi}-\mu\left(\left.\frac{\partial v_{\xi}}{\partial \zeta}\right|_{f_{2}}-\left.\frac{\partial v_{\xi}}{\partial \zeta}\right|_{f_{1}}\right)+\rho \widetilde{v_{\xi}}\left(\left.v_{\eta}\right|_{f_{2}}-\left.v_{\eta}\right|_{f_{1}}\right)-\mu \frac{\partial}{\partial \xi}\left(\left.v_{\eta}\right|_{f_{2}}-\left.v_{\eta}\right|_{f_{1}}\right)=0,
$$

which reduces for uniform leakage over the fracture length, $\left.v_{\eta}\right|_{f_{2}}=\left.v_{\eta}\right|_{f_{1}}=q_{l}$, to

$$
\rho g b \frac{d \widetilde{h}}{d \xi}-\mu\left(\left.\frac{\partial v_{\xi}}{\partial \zeta}\right|_{f_{2}}-\left.\frac{\partial v_{\xi}}{\partial \zeta}\right|_{f_{1}}\right)+2 \rho \widetilde{v_{\xi}} q_{l}=0
$$


We then assume that the velocity in the fracture is much larger than the leakage rate into the walls, so that the no-slip boundary conditions can still be employed, and arrive at the modified equation

$$
\widetilde{v_{\xi}}=-\frac{\rho g b^{2}}{\mu+2 b \rho q_{l}} \frac{d \widetilde{h}}{d \eta} .
$$

Finally, the variable aperture of a fracture can be taken into account (Wilson and Witherspoon, 1974), which introduces the concept of the effective aperture

$$
b_{\text {eff }}^{3}=\frac{\sum_{i=1}^{m} l_{i}}{\sum_{i=1}^{m}\left(l_{i} / b_{i}^{3}\right)}
$$

for $m$ discrete segments with different apertures $b_{i}$ and lengths $l_{i}$. The harmonic-type mean $b_{\text {eff }}$ is then used in A.I3. 
A. The Hele-Shaw Analog for Viscous Flow in Parallel Plates 


\section{B. INTEGRAL TRANSFORMATIONS}

In this section we explain how the evaluation of integrals on the reference element is done for lower dimensional elements. The transformation includes only basic vector calculus, but we include the subject since it is seldomly treated in standard finite element literature. As a reminder we repeat how the transformation to the reference element is done in the case of triangular elements in $\mathbb{R}^{2}$. The transformation to the reference elements uses standard results from vector analysis as they can be found in Jänich (2003) or Marsden and Tromba (1996).

\section{B.I. TWO-DIMENSIONAL ELEMENTS IN TWO SPACE DIMENSIONS}

We consider a function $u$ on a fracture element $\Omega_{e}$ and the mapping $T$ which maps the reference element $\hat{\Omega}_{e}$ to the element in $\mathbb{R}^{2}$. The mapping $T$ is an affine mapping of the form

$$
T(\xi, \eta)=B\left(\begin{array}{l}
\xi \\
\eta
\end{array}\right)+d=\left(\begin{array}{ll}
x_{1}-x_{0} & x_{2}-x_{0} \\
y_{1}-y_{0} & y_{2}-y_{0}
\end{array}\right)\left(\begin{array}{l}
\xi \\
\eta
\end{array}\right)+\left(\begin{array}{l}
x_{0} \\
y_{0}
\end{array}\right)
$$

and the derivatives are transformed as

$$
D_{\xi, \eta} u(F(\xi, \eta))=D_{x, y} u(F(\xi, \eta)) \cdot D_{\xi, \eta} F(\xi, \eta)=D_{x, y} u(F(\xi, \eta)) \cdot B
$$

by the chain rule, where $D_{x, y}$ and $D_{\xi, \eta}$ are the row vectors $\left(\partial_{x}, \partial_{y}\right)$ and $\left(\partial_{\xi}, \partial_{\eta}\right)$, respectively. The gradient of $u$ in $x, y$-coordinates is then

$$
\nabla_{x, y} u(F(\xi, \eta))=\left(B^{-1}\right)^{\top} \nabla_{\xi, \eta} F(\xi, \eta) .
$$

This is used in the evaluation of the integral over the element $\Omega_{e}$ by transformation to the reference element $\hat{\Omega}_{e}$ where we write $\mathrm{B}^{-\mathrm{T}}$ for $\left(\mathrm{B}^{-1}\right)^{\mathrm{T}}$.

$$
\begin{aligned}
\int_{\Omega_{e}} \nabla_{x, y} u \cdot \nabla_{x, y} v d x d y & =\int_{\hat{\Omega}_{e}} \nabla_{x, y} u(F(\xi, \eta)) \cdot \nabla_{x, y} v(F(\xi, \eta))\left|\operatorname{det}\left(D_{\xi, \eta} F(\xi, \eta)\right)\right| d \xi d \eta \\
& =\int_{\hat{\Omega}_{e}} B^{-T} \nabla_{\xi, \eta} u(F(\xi, \eta)) \cdot B^{-T} \nabla_{\xi, \eta} v(F(\xi, \eta))|\operatorname{det}(B)| d \xi d \eta \\
& =\int_{\hat{\Omega}_{e}} B^{-T} \nabla_{\xi, \eta} \hat{u}(\xi, \eta) \cdot B^{-T} \nabla_{\xi, \eta} \hat{v}(\xi, \eta)|\operatorname{det}(B)| d \xi d \eta \\
& =\int_{\hat{\Omega}_{e}}\left(B^{T} B\right)^{-1} \nabla_{\xi, \eta} \hat{u}(\xi, \eta) \cdot \nabla_{\xi, \eta} \hat{v}(\xi, \eta)|\operatorname{det}(B)| d \xi d \eta
\end{aligned}
$$




\section{B. INTEGRAL TRANSFORMATIONS}

\section{B.2. ONE-DIMENSIONAL ELEMENTS IN TWO SPACE DIMENSIONS}

Let us now consider the transformation of a line element into $\mathbb{R}^{2}$. The transformation in this case is

$$
\mathrm{T}(\xi)=B(\xi)+\mathrm{d}=\left(\begin{array}{l}
x_{1}-x_{0} \\
y_{1}-y_{0}
\end{array}\right)(\xi)+\left(\begin{array}{l}
x_{0} \\
y_{0}
\end{array}\right),
$$

and the chain rule applies again,

$$
\hat{u}^{\prime}=u^{\prime}(B \xi+d) \cdot T^{\prime}(\xi)=\left(\partial_{x} u, \partial_{y} u\right) \cdot B=B^{\top} \cdot \nabla u .
$$

(We write $\nabla u$ instead of $\nabla_{x, y} u$.) Like in the example above, $\nabla_{x, y} u=B^{-\top} \hat{u}^{\prime}$ holds, but since $B$ is not a square matrix it is not obvious what the inversion means. A geometrically motivated way to determine $\mathrm{B}^{-\mathrm{T}}$ is as follows. The linear system is underdetermined and can be extended in a meaningful way. $\mathrm{B}^{\mathrm{T}} \cdot \nabla \mathrm{u}$ is the directional derivative in direction $\mathrm{B}^{\mathrm{T}}$, i. e. the fracture direction. The function $u(x, y)$ should have no variation in the direction orthogonal to the fracture direction. This leads to the additional requirement

$$
0=\left(\partial_{x} u, \partial_{y} u\right) \cdot N=\left(\partial_{x} u, \partial_{y} u\right) \cdot\left(\begin{array}{c}
y_{1}-y_{0} \\
-\left(x_{1}-x_{0}\right)
\end{array}\right) \text {. }
$$

Together, equations $\mathrm{B.I}$ and $\mathrm{B.2}$ state

$$
\left(\begin{array}{c}
\hat{u}^{\prime} \\
0
\end{array}\right)=\left(\begin{array}{cc}
x_{1}-x_{0} & y_{1}-y_{0} \\
y_{1}-y_{0} & -\left(x_{1}-x_{0}\right)
\end{array}\right)\left(\begin{array}{c}
\partial_{x} u \\
\partial_{y} u
\end{array}\right)=\tilde{A}\left(\begin{array}{c}
\partial_{x} u \\
\partial_{y} u
\end{array}\right) \text {. }
$$

The inverse of $\tilde{A}$ is

$$
\tilde{A}^{-1}=\frac{1}{\left(x_{1}-x_{0}\right)^{2}+\left(y_{1}-y_{0}\right)^{2}}\left(\begin{array}{cc}
x_{1}-x_{0} & y_{1}-y_{0} \\
y_{1}-y_{0} & -\left(x_{1}-x_{0}\right)
\end{array}\right)
$$

and it follows that

$$
\left(\begin{array}{l}
\partial_{x} u \\
\partial_{y} u
\end{array}\right)=\frac{1}{\left(x_{1}-x_{0}\right)^{2}+\left(y_{1}-y_{0}\right)^{2}}\left(\begin{array}{l}
x_{1}-x_{0} \\
y_{1}-y_{0}
\end{array}\right) \hat{u}^{\prime}=B^{+} \hat{u}^{\prime}
$$

Note that we write $\mathrm{B}^{+}$for the generalized inverse of $\mathrm{B}$. It is no coincidence that this notation resembles the Moore-Penrose pseudo inverse: $\mathrm{B}^{+}$is the pseudo inverse of $\mathrm{B}$ (Horn and Johnson, I985, I99I). The connection is that $A^{+} A x \in(\operatorname{ker} A)^{\perp}$ (the orthogonal complement of the kernel of A).

For the integral transformation of lines we know from vector analysis that for $\mathrm{T}: \hat{\omega}_{e} \rightarrow$ $\omega_{e}, \hat{\omega}_{e}=(0,1), \omega_{e} \subset \mathbb{R}^{2}$ the integral transforms as

$$
\int_{\Omega} f(s) d s=\int_{\omega} f(T(\xi))\left\|T^{\prime}(\xi)\right\| d \xi=\int_{\omega} f(T(\xi)) \sqrt{\left(x_{1}-x_{0}\right)^{2}+\left(y_{1}-y_{0}\right)^{2}} d \xi .
$$




\section{B.3. LOWER-DIMENSIONAL ELEMENTS IN THREE SPACE DIMENSIONS}

For two-dimensional triangular elements the transformation rule is

$$
\int_{\omega} f(x, y) d F=\int_{\hat{\omega}} f(T(\xi, \eta))\left\|\frac{\partial T}{\partial \xi} \times \frac{\partial T}{\partial \eta}\right\| d \xi d \eta
$$

The transformation is given by

$$
T(\xi, \eta)=B(\xi, \eta)+d=\left(\begin{array}{ll}
x_{1}-x_{0} & x_{2}-x_{0} \\
y_{1}-y_{0} & y_{2}-y_{0} \\
z_{1}-z_{0} & z_{2}-z_{0}
\end{array}\right)(\xi, \eta)+\left(\begin{array}{l}
x_{0} \\
y_{0} \\
z_{0}
\end{array}\right)
$$

The derivation of $\mathrm{B}^{-\mathrm{T}}$ can be done in the geometrically motivated way as before by considering the matrix $C$,

$$
(\mathbf{a}, \mathbf{b}):=\left(\begin{array}{ll}
x_{1}-x_{0} & x_{2}-x_{0} \\
y_{1}-y_{0} & y_{2}-y_{0} \\
z_{1}-z_{0} & z_{2}-z_{0}
\end{array}\right) \quad C=(\mathbf{a}, \mathbf{b}, \mathbf{a} \times \mathbf{b})
$$

Calculating $\mathrm{C}$ and selecting the first two columns yields the pseudo inverse $\mathrm{B}^{+}$.

The integral transformation for one-dimensional elements in $\mathbb{R}^{3}$, which are transformed from the reference element $(0,1)$ by $\mathrm{T}: \xi \mapsto(x, y, z)$, is

(B.9) $\int_{\omega} f(s) d s=\int_{\hat{\omega}} f(T(\xi))\left\|T^{\prime}(\xi)\right\| d \xi=\int_{\hat{\omega}} f(T(\xi)) \sqrt{\left(x_{1}-x_{0}\right)^{2}+\left(y_{1}-y_{0}\right)^{2}+\left(z_{1}-z_{0}\right)^{2}} d \xi$.

The derivation of the pseudo-inverse is done as before, except that we have to find two normal directions to the fracture direction. 
B. INTEGRAL TRANSFORMATIONS 


\section{BIBLIOGRAPHY}

D. Abdassah and I. Ershaghi. Triple-porosity system for representing naturally fractured reservoirs. SPE Form. Eval., I:II3-I27, 1986.

P. M. Adler and J.-F. Thovert. Fractures and Fracture Networks. Kluwer Acad., Norwell, Mass., I999.

V. Aizinger, C. Dawson, B. Cockburn, and P. Castillo. The local discontinuous Galerkin method for contaminant transport. Adv. Wat. Res., 24:73-87, $200 \mathrm{I}$.

R. Alexander. Diagonally implicit Runge-Kutta methods for stiff O.D.E's. SIAM J. Sci. Comput., I4:I006-IO2I, I977.

G. Allaire. One-Phase Newtonian Flow. In U. Hornung, editor, Homogenization and Porous Media, volume 6 of Interdisciplinary Applied Mathematics. Springer-Verlag, Berlin, Heidelberg, New York, 1997. ISBN 0-387-94786-8.

T. Arbogast. Analysis of the simulation of single phase flow through a naturally fractured reservoir. SLAM J. Numer. Anal., 26:I2-29, 1989.

T. Arbogast. Computational Aspects of Dual-Porosity Models. In U. Hornung, editor, Homogenization and Porous Media, volume 6 of Interdisciplinary Applied Mathematics. Springer-Verlag, Berlin, Heidelberg, New York, 1997. ISBN 0-387-94786-8.

T. Arbogast, J. Douglas jr., and U. Hornung. Derivation of the double porosity model of single phase flow via homogenization theory. SIAM J. Math. Anal., 21:823-836, 1990.

T. Arbogast, J. Douglas jr., and U. Hornung. Modeling of naturally fractured petroleum reservoirs by formal homogenization techniques. In R. Daurtay, editor, Frontiers in Pure and Applied Mathematics, pages I-I9. Elsevier, Amsterdam, I99I.

D. Arnold. An interior penalty finite element method with discontinuous elements. $\mathrm{PhD}$ thesis, University of Chicago, 1979.

D. Arnold. An interior penalty finite element method with discontinuous elements. SIAM J. Numer. Anal., I9(4):742-760, 1982.

D. Arnold, F. Brezzi, B. Cockburn, and L. Marini. Unified analysis of discontinuous Galerkin methods for elliptic problems. SLAM J. Numer. Anal., 39(5):I749-1779, 2002.

R. G. Baca, R. C. Arnett, and I. P. King. Modeling of fluid flow in fractured-porous rock masses by finite-element techniques. Int. J. Num. Meth. Fluids, 4:337-348, 1984. 


\section{BIBLIOGRAPHY}

R. Bank and C. Wagner. Multilevel ILU decomposition. Numerische Mathematik, 82:543576, 1999.

R. E. Bank and D. J. Rose. Some error estimates for the box scheme. SIAM J. Numer. Anal., 24:777-787, 1987.

G. I. Barenblatt, I. P. Zheltov, and I. N. Kochina. Basic concepts in the theory of seepage of homogeneous liquids in fissured rocks (strata). Journal of Applied Mathematical Mechanics (USSR), 24:1286-I303, 1960.

R. Barrett, M. Berry, T. Chan, J. Demmel, J. Donato, J. Dongarra, V. Eijkhout, R. Pozo, C. Romine, and H. van der Vorst. Templates for the Solution of Linear Systems: Building Blocks for Iterative Methods. SIAM, 1994.

C. C. Barton, E. Larson, W. R. Page, and T. M. Howard. Characterising fractured rock for fluid flow, geochemical, and paleostress modelling: Methods and preliminary results from Yucca Mountain, Nevada, 1987.

F. Bassi and R. Rebay. A high-order accurate discontinuous finite element method for the numerical solution of the compressible Navier-Stokes equations. J. Comput. Phys., IзI: 267-279, 1997a.

F. Bassi and S. Rebay. High-order accurate discontinuous finite element solution of the $2 \mathrm{D}$ Euler equations. J. Comput. Phys., I38:25I-285, I997b.

F. Bassi and S. Rebay. GMRES Discontinuous Galerkin Solution of the Compressible Navier-Stokes Equations. In B. Cockburn, G. E. Karniadakis, and C. Shu, editors, Discontinuous Galerkin Methods: Theory, Computation and Applications, volume II of Lecture Notes in Computational Science and Engineering. Springer-Verlag, Berlin, Heidelberg, New York, 2000.

P. Bastian. Parallele adaptive Mehrgitterverfahren. Teubner-Verlag, 1996.

P. Bastian. Numerical Computation of Multiphase Flow in Porous Media, 1999. Habilitationsschrift, Universität Kiel.

P. Bastian. Higher Order Discontinuous Galerkin Methods for Flow and Transport in Porous Media. Technical Report 2002-33, IWR (SFB 359), Universität Heidelberg, 2002. to appear in Lecture Notes in Computational Science and Engineering.

P. Bastian, K. Birken, S. Lang, K. Johannsen, N. Neuß, H. Rentz-Reichert, and C. Wieners. UG: A flexible software toolbox for solving partial differential equations. Computing and Visualization in Science, I:27-40, 1997. http://cox.iwr.uni-heidelberg.de/ ug.

P. Bastian and R. Helmig. Efficient fully-coupled solution techniques for two-phase flow in porous media. Parallel multigrid solution and large scale computations. Adv. Water Res., 23:199-2I6, I999. 


\section{BIBLIOGRAPHY}

P. Bastian and S. Lang. Couplex Benchmark Computations with UG. Technical Report 2002-3I, IWR (SFB 359), Universität Heidelberg, 2002. to appear in Computational Geosciences.

P. Bastian and V. Reichenberger. Multigrid for higher order discontinuous Galerkin finite elements applied to groundwater flow. Technical report, SFB 359, Universität Heidelberg, 2000. Preprint 2000-37.

C. Baumann. An hp-adaptive discontinuous finite element method for computational fluid dynamics. PhD thesis, The University of Texas at Austin, 1997.

C. E. Baumann and J. T. Oden. A discontinuous hp finite element method for convectiondiffusion problems. Comput. Methods Appl. Mech. Engrg., I75:3II-34I, I999.

J. Bear. Dynamics of Fluids in Porous Media. Dover Publications Inc., New York, 1972.

J. Bear. Modeling Flow and Contaminant Transport in Fractured Rocks. In J. Bear, C.-F. Tsang, and G. de Marsily, editors, Flow and contaminant transport in fractured rock, pages I-37. Academic Press, Inc., San Diego, 1993.

J. Bear, C.-F. Tsang, and G. de Marsily, editors. Flow and contaminant transport in fractured rock. Academic Press, Inc., San Diego, I993. ISBN 0-I2-083980-6.

B. Berkowitz. Characterizing flow and transport in fractured geological media: A review. Advances in Water Resources, 25:86I-884, 2002.

B. Berkowitz, J. Bear, and C. Braester. Continuum models for contaminant transport in fractured porous formations. Water Resources Research, 24(8):I225-I236, 1988.

S. P. Bertels, D. A. DiCarlo, and M. J. Blunt. Measurement of aperture distribution, capillary pressure, relative permeability, and in situ saturation in a rock fracture using computed tomography scanning. Water Resources Research, 37(3):649-662, $3200 \mathrm{I}$.

J. Bey. Finite-Volumen- und Mehrgitter-Verfahren für elliptische Randwertprobleme. B. G. Teubner, Stuttgart, Leipzig, I998. ISBN 3-519-0274I-O.

J. Bey and G. Wittum. Downwind numbering: Robust multigrid for convection-diffusion problems. Appl. Numer. Math., 23:I77-192, 1997.

P. Binning and M. Celia. Eulerian-Lagrangian localized adjoint methods for contaminant transport simulations. In A. P. et al., editor, Computational Methods in Water Resources X. Kluwer Academic Publishers, 1994.

A. Bourgeat. Two-Phase Flow. In U. Hornung, editor, Homogenization and Porous Media, volume 6 of Interdisciplinary Applied Mathematics. Springer-Verlag, Berlin, Heidelberg, New York, 1997. ISBN 0-387-94786-8.

D. Braess. Finite Elemente. Springer-Lehrbuch. Springer-Verlag, Berlin, Heidelberg, New York, 1992. 


\section{BIBLIOGRAPHY}

D. Braess. Towards algebraic multigrid for elliptic problems of second order. Computing, 55 : 379-393, 1995.

F. Brakhagen and T. Fogwell. Multigrid for the fully implicit formulation of the equations for multiphase flow in porous media. In Multigrid Methods: Special topics and applications, volume II, pages 3I-42, I990.

J. Bramble, J. Pasciak, J. Wang, and J. Xu. Convergence estimates for multigrid algorithms without regularity assumptions. Math. Comput., 57:I-22, I99I.

J. H. Bramble. Multigrid Methods. Number 294 in Pitman Research Notes in Mathematics. Longman, Essex, 1993. ISBN 0-582-23435-2.

S. C. Brenner and L.-Y. Sung. Multigrid method for the computation of singular solutions and stress intensity factors. II: Crack singularities. BIT, 37:623-643, 1997.

W. L. Briggs, V. E. Henson, and S. F. McCormick. A Multigrid Tutorial. SIAM Books, Philadelphia, second edition, 2000.

H. C. Brinkman. A calculation of the viscous force exerted by a flowing fluid on a dense swarm of particles. Appl. Sci. Res., A I:27-34, 1947.

A. Brooks and T. Hughes. Streamline upwind/Petrov-Galerkin formulations for convection dominated flows with particular emphasis on the incompressible Navier-Stokes equation. Computer Methods in Applied Mechanics and Engineering, 32:199-259, I982.

P. Castillo. Performance of discontinuous galerkin methods for elliptic pdes. SIAM Journal on Scientific Computing, 24(2):524-547, 2002.

M. Celia, T. Russel, I. Herrera, and R. Ewing. An Eulerian-Lagrangian localized adjoint method for the advection-diffusion equation. Adv. Water Resources, I3(4):187-206, 1990.

G. Chavent. A new formulation of diphasic incompressible flows in porous media. volume 503 of Lecture Notes in Mathematics, pages 258-270. Springer-Verlag, Berlin, Heidelberg, New York, 1976.

G. Chavent. The global pressure, a new concept for mobilization of compressible two phase flow in porous media. In A. Verruyt and F. B. J. Barends, editors, Flow and Transport in Porous Media. Balkema, Rotterdam, I98I.

G. Chavent and J. Jaffré. Mathematical Models and Finite Elements for Reservoir Simulation. North-Holland, New York, 1978.

Z. Chen, B. Cockburn, C. Gardner, and J. Jerome. Quantum hydrodynamic simulation of hysteresis in the resonant tunneling diode. J. Comput. Phys., II7:274-280, I995a.

Z. Chen, B. Cockburn, J. Jerome, and C. Shu. Mixed-RKDG finite element methods for the 2-D hydrodynamic model for semiconductor device simulation. VLSI Design, 3:I45-I58, I995b. 


\section{BIBLIOGRAPHY}

Z.-X. Chen. Transient flow of slightly compressible fluids through double-porosity, doublepermeability systems - a state-of-the-art review. Transport in Porous Media, 4(2):I47-I84, 1989.

P. Ciarlet. The finite element method for elliptic problems. North-Holland, New York, 1978. Republished as vol. 40 in Classics in Applied Mathematics, Society for Industrial \& Applied Mathematics. Philadelphia, 2002.

H. Class. Theorie und numerische Modellierung nichtisothermer Mehrphasenprozesse in NAPLkontaminierten porösen Medien. PhD thesis, Mitteilungsheft Io5, Institut für Wasserbau, Universität Stuttgart, 200I.

T. M. Clemo and L. Smith. Solute transport in fractured media: Dual permeability models. EOS, $70(43)$, 1989.

B. Cockburn. An Introduction to the Discontinuous Galerkin Method for ConvectionDiffusion Laws. In B. Cockburn, C. Johnson, C.-W. Shu, and E. Tadmor, editors, Advanced Numerical Approximation of Nonlinear Hyperbolic Equations, volume 1687 of Lecture Notes in Mathematics, pages I5I-268. Springer-Verlag, Berlin, Heidelberg, New York, I998.

B. Cockburn. Discontinuous Galerkin Methods for Convection-Dominated Problems. In T. J. Barth and H. Deconinck, editors, High-Order Methods for Computational Physics, volume 9 of Lecture Notes in Computational Engineering, pages 69-225. Springer-Verlag, Berlin, Heidelberg, New York, I999.

B. Cockburn, S. Hou, and C. Shu. TVB Runge-Kutta local projection discontinuous Galerkin finite element method for conservation laws III: One-dimensional systems. J. Comput. Phys., 84:90, 1989.

B. Cockburn, S. Hou, and C. Shu. TVB Runge-Kutta local projection discontinuous Galerkin finite element method for conservation laws IV: The multidimensional case. Math. Comput., 54:545, 1990.

B. Cockburn, G. Karniadakis, and C. Shu. The Development of Discontinuous Galerkin Methods. In B. Cockburn, G. E. Karniadakis, and C. Shu, editors, Discontinuous Galerkin Methods: Theory, Computation and Applications, volume II of Lecture Notes in Computational Science and Engineering, pages 3-50. Springer-Verlag, Berlin, Heidelberg, New York, 2000 .

B. Cockburn, G. E. Karniadakis, and C. Shu, editors. Discontinuous Galerkin Methods: Theory, Computation and Applications, volume II of Lecture Notes in Computational Science and Engineering. Springer-Verlag, Berlin, Heidelberg, New York, 2ooob.

B. Cockburn, G. E. Karniadakis, and C. Shu, editors. Discontinuous Galerkin Methods: Theory, Computation and Applications, volume iI of Lecture Notes in Computational Science and Engineering. Springer-Verlag, Berlin, Heidelberg, New York, 200oc. 


\section{BIBLIOGRAPHY}

B. Cockburn and C. Shu. TVB Runge-Kutta local projection discontinuous Galerkin finite element method for conservation laws II: Gerneral framework. Math. Comput., 52(I86): 4II-435, 1989 .

B. Cockburn and C. Shu. The Runge-Kutta local projection $\mathrm{P}^{1}$-discontinuous Galerkin method for scalar conservation laws. $M^{2} \mathrm{AN}, 25: 337$, I99I.

B. Cockburn and C. Shu. The local discontinuous Galerkin finite element method for convection-diffusion systems. SIAM J. Numer. Anal., 35:2440-2463, I998a.

B. Cockburn and C. Shu. The Runge-Kutta discontinuous Galerkin method for conservation laws V: Multidimensional systems. J. Comput. Phys., I4I:I99-224, I998b.

R. Courant, K. O. Friedrichs, and H. Lewy. Über die partiellen Differentialgleichungen der mathematischen Physik. Math. Ann., I00:32-74, I928.

COVISE. Collaborative Visualization and Simulation Environment. http://www.hlrs. de/organization/vis/covise/.

C. F. Curtiss and J. O. Hirschfelder. Integration of stiff equations. Proc. Nat. Acas. Sci., 38 : $235-243$, 1952 .

H. Darcy. Les Fontaines Publiques de la Vilee de Dijon. Dalmont, Paris, I856.

R. A. Dawe, M. R. Wheat, and M. S. Bidner. Experimental investigation of capillary pressure effects on immiscible displacement in lensed and layered porous media. Transport in porous media., 7(9):83-IOI, I992.

M. Delfour, W. Hager, and F. Trochu. Discontinuous Galerkin methods for ordinary differential equations. Math. Comp., 36:453-473, I98I.

J. Dendy Jr. Two multigrid methods for three-dimensional problems with discontinuous and anisotropic coefficients. SIAM J. Sci. Stat. Comput., 8:673-685, 1987.

C. R. Doering and J. D. Gibbon. Applied Analysis of the Navier-Stokes Equations. Cambridge Texts in Mathematics. Cambridge University Press, 1995.

J. Douglas jr. Finite difference methods for two-phase incompressible flow in porous media. SIAM J. Numer. Anal., 20(4):68I-696, August 1983.

J. Douglas Jr., D. Peaceman, and H. Rachford Jr. A method for calculating multidimensional displacement. Trans. AIME, 216:297-308, 1959.

J. Douglas Jr. and T. Russel. Numerical methods for convection dominated diffusion problems based on combining the method of characteristics with finite element or finite difference procedures. SIAM J. Numer. Anal., I9(5):87I-885, 1982.

M. Dryja, M. Sarkin, and O. Widlund. Multilevel Schwartz methods for elliptic problems with discontinuous coefficients in three space dimensions. Numer. Math., 72:313-348, I996. 


\section{BIBLIOGRAPHY}

L. Durlofsky. A triangle based mixed finite element-finite volume technique for modeling two-phase flow through porous media. Journal of Computational Physics, I05:252-266, 1993.

L. Durlofsky. Accuracy of mixed and control volume finite element approximations to Darcy velocity and related quantities. Water Resources Research, 30(4):965-973, 1994.

R. R. Eaton and N. E. Bixler. Analysis of a multiphase, porous-flow imbibition experiment in a fractured volcanic tuff. In D. D. Evans, editor, Flow and Transport through Unsaturated Fractured Rock, pages 9I-97. Geophysical Monograph 42, 1987.

K. Eriksson, D. Estep, P. Hansbo, and C. Johnson. Computational Differential Equations. Cambridge University Press, 1996.

K. Eriksson, C. Johnson, and V. Thomeée. Time discretization of parabolic problems by the discontinuous Galerkin method. RAIRO Modél. Math. Anal. Numér., I9:6II-643, I985.

L. C. Evans. Partial Differential Equations, volume 19 of Graduate Studies in Mathematics. American Mathematical Society, Providence, Rhode Island, I998.

R. Ewing. Operator splitting and Eulerian-Lagrangian localized adjoint methods for multiphase flow. In The Mathematics of Finite Elements and Applications VII, I99I.

R. E. Ewing. Problems arising in the modeling of processes for hydrocarbon recovery. In Richard. E. Ewing, editor, Research Frontiers in Applied Mathematics, volume I, pages 3-34. SIAM, I983.

P. Forchheimer. Wasserbewegung durch Boden. Zeit. Ver. deut. Ing., 45:I78I- I788, I90I.

P. Forsyth. A control volume finite element approach to NAPL groundwater contamination. SIAM J. Sci. Stat. Comput., I2(5):I029-I057, I99I.

A. Fuchs. Optimierte Delaunay-Triangulierungen zur Vernetzung getrimmter NURBS-Körper. Shaker Verlag, 1999.

J. Glimm, E. Isaacson, B. Lindquist, O. McBryan, and S. Yaniv. Statistical fluid dynamics: The influence of geometry on surface instabilites. In R. Ewing, editor, Research Frontiers in Applied Mathematics, volume I, pages I37-I60. SIAM, I983.

J. Glimm, D. Marchesin, and O. McBryan. Unstable fingers in two phase flow. Comm. Pure Appl. Math., 34:53-75, 198I.

G. H. Golub and C. F. van Loan. Matrix Computations. Johns Hopkins Series in the Mathematical Sciences. The Johns Hopkins University Press, Baltimore, Maryland, third edition, 1996.

J. Gopalakrishnan and G. Kanschat. A multilevel discontinuous Galerkin method. Numerische Mathematik, 95(3):527-550, September 2003. ISSN 0029-599X. 


\section{BIBLIOGRAPHY}

W. G. Gray and P. C. Y. Lee. On the theorems for local volume averaging of multiphase systems. International Journal of Multiphase Flow, 3:333-340, 1977.

A. B. Gureghian. A study by the finite element method of the influence of fractures in confined aquifers. Soc. Pet. Eng. J., I5:I8I-I9I, I975.

W. Hackbusch. Multi-Grid Methods and Applications. Springer-Verlag, Berlin, Heidelberg, New York, 1985.

W. Hackbusch. On First and Second Order Box Schemes. Computing, 4I:277-296, I989.

W. Hackbusch. Iterative Solution of Large Sparse Systems of Linear Equations. Springer, New York, 1994.

W. Hackbusch. On the feedback vertex set problem for a planar graph. Computing, 58(2): I29-I55, 1997.

E. Hairer and G. Wanner. Solving ordinaray differential equations II. Stiff and differential algebraic problems, volume I4 of Springer Series in Computational Mathematics. SpringerVerlag, Berlin, Heidelberg, New York, 2 edition, 1996. ISBN 3-540-60452-9.

R. Helmig. Theorie und Numerik der Mehrphasenströmungen in geklüftet-porösen Medien. $\mathrm{PhD}$ thesis, Institut für Strömungsmechanik und Elektronisches Rechnen im Bauwesen, Universität Hannover, Bericht Nr. 34, 1993.

R. Helmig. Multiphase Flow and Transport Processes in the Subsurface. A Contribution to the Modeling of Hydrosystems. Springer-Verlag, Berlin, Heidelberg, New York, 1997.

R. Helmig and R. Huber. Multiphase flow in heterogeneous porous media: A classical finite element method versus an IMPES-based mixed FE/FV approach. Technical Report I9, Sonderforschungsbereich 404, Universität Stuttgart, 1996. to appear in Int. J. Numer. Meth. in Fluids.

P. W. Hemker, W. Hoffmann, and M. H. van Raalte. Fourier two-level analysis for discontinuous Galerkin discretization with linear elements. Technical Report MAS-Ro2I7, Centrum voor Wiskunde en Informatica (CWI), Modelling, Analysis and Simulation (MAS), Amsterdam, 2002a. URL www. cwi.nl/ftp/CWIreports/MAS/MAS-R0217.pdf.

P. W. Hemker, W. Hoffmann, and M. H. van Raalte. Two-level Fourier analysis of a multigrid approach for discontinuous Galerkin discretisation. Technical Report MAS-Ro206, Centrum voor Wiskunde en Informatica (CWI), Modelling, Analysis and Simulation (MAS), Amsterdam, 2002b. URL www.cwi.nl/ftp/CWIreports/MAS/MAS-R0206 .pdf. submitted to SIAM SISC.

P. W. Hemker and M. H. van Raalte. Fourier two-level analysis for higher dimensional discontinuous Galerkin Discretisation. Technical Report MAS-Ro227, Centrum voor Wiskunde en Informatica (CWI), Modelling, Analysis and Simulation (MAS), Amsterdam, 2002. URL www. cwi.nl/ftp/CWIreports/MAS/MAS-R0227.pdf. 


\section{BIBLIOGRAPHY}

A. Hill and G. Thomas. A New Approach for Simulating Complex Fractured Reservoirs. In Reservoir Simulation Symposium, Dallas, 1985.

R. A. Horn and C. R. Johnson. Matrix Analysis. Cambridge University Press, 1985.

R. A. Horn and C. R. Johnson. Topics in Matrix Analysis. Cambridge University Press, I99I.

U. Hornung, editor. Homogenization and Porous Media, volume 6 of Interdisciplinary Applied Mathematics. Springer-Verlag, Berlin, Heidelberg, New York, 1997. ISBN 0-387-94786-8.

T. Y. Hou and P. G. LeFloch. Why nonconservative schemes converge to wrong solutions: Error analysis. Mathematics of Computation, 62(206):497-530, April 1994.

C. Hu and C. Shu. A discontinuous galerkin finite element method for hamilton-jacobi equations. SIAM J. Sci. Comput., 2000.

O. Ippisch. Coupled Transport in Natural Porous Media. PhD thesis, Universität Heidelberg, 200 I.

J. S. Y. Wang and T. N. Narasimhan. Hydrologic mechanisms governing fluid flow in a partially saturated fractured prous medium. Water Resources Research, 2I(I2):I86I-I874, 1985.

H. Jakobs. Nicht-isotherme Gas-Wasser-Prozesse in stark heterogenen Medien. PhD thesis, Universität Stuttgart, Stuttgart, 2003.

H. Jakobs, R. Helmig, C. Miller, H. Class, M. Hilpert, and C. Kees. Modeling of DNAPL flow in saturated heterogeneous porous media. To appear in Advances in Water Resources, 2003.

P. Jamet. Galerkin-type approximations which are discontinuous in time for parabolic equations in a variable domain. SIAM J. Numer. Anal., I5:912-928, 1978.

K. Jänich. Vektoranalysis. Springer-Lehrbuch. Springer-Verlag, Berlin, Heidelberg, New York, 4 edition, 2003. ISBN 3-540-00392-4.

J. Jarsjö and J. T. Geller. Groundwater degassing: Laboratory experiments in rock fracture replicas with radial flow. SKB Äspö HRL Progress Report, Swedish Nuclear Fuel and Waste Management Company, 1996.

C. Johnson, Y. Nie, and V. Thomeée. Time discretization of parabolic problems by the discontinuous Galerkin method. SIAM J. Numer. Anal., 27:277-291, I990.

H. Kazemi. Pressure Transient Analysis of Naturally Fractured Reservoirs. Trans. AIME, 256:45I-46I, I969.

D. Kroener and S. Luckhaus. Flow of oil and water in a porous medium. Journal of Differential Equations, 55:276-288, 1984. 


\section{BIBLIOGRAPHY}

B. Kueper and E. Frind. An overview of immiscible fingering in porous media. Journal of Contaminant Hydrology, 2:95-IIO, I988.

B. H. Kueper, W. Abbott, and G. Farquhar. Experimental Observations of Multiphase Flow in Heterogeneous Porous Media. Journal of Contaminant Hydrology, 5:83-95, 1989.

S. Lang. Parallele Numerische Simulation instationärer Probleme mit adaptiven Methoden auf unstrukturierten Gittern. PhD thesis, Universität Stuttgart, 2000.

S. Laubach. Fracture networks in selected Cretaceous sandstones of the Green River and San Juan Basin, Wyoming, New Mexico, and Colorado. In J. M. Schmoker, E. B. Coalsen, and C. A. Brown, editors, Geological studies relevant to horizontal drilling: Examples from Western North America, pages 6I-73. Rocky Mountain Assoc. of Geologists, 1992.

R. J. Lenhard, J. C. Parker, and S. Mishra. On the correspondence between Brooks-Corey and van Genuchten models. J. Irrig. Drain. Eng., II5:744-75I, 1989.

P. Lesaint and P. Raviart. On a finite element method for solving the neutron transport equation. In C. de Boor, editor, Mathematical Aspects of Finite Elements in Partial Differential Equations, pages 89-I23. Academic Press, new York, I974.

R. J. Le Veque. Numerical Methods for Conservation Laws. Lectures in Mathematics: ETH Zürich. Birkhäuser Verlag, Basel, Boston, Berlin, I992. ISBN 3-7643-2723-5.

R. J. Le Veque. Finite Volume Methods for Hyperbolic Problems. Cambridge Texts in Applied Mathematics. Cambridge University Press, Cambridge, 2002. ISBN 0-52I-00924-3.

J. Lions. Problèmes aux limites non homogènes à donées irrégulières: Une méthode d'approximation. In Numerical Analysis of Partial Differential Equations (C.I.M.E. 2 Ciclo, Ispra, 1967), pages 283-292. Rome, I968. Edizioni Cremonese.

G. M. Lomize. Flow in fractured rocks (in russian). Gosenergoizdat, Moscow, I95I.

C. Louis. A study of groundwater flow in jointed rock and its influence on the stability of rock masses. Rock Mech. Res. Rep., Imp. Coll., I0:90 pp., 1969.

F. J. Lucia. Carbonate Reservoir Characterization. Springer-Verlag, Berlin, Heidelberg, New York, 1999. ISBN 3-540-63782-6.

Q. Ma, J. E. Hook, and L. R. Ahuja. Influence of three-parameter conversion methods between van Genuchten and Brooks-Corey functions on soil hydraulic properties and waterbalance predictions. Water Resources Research, 35(8):257I-2578, August 1999.

J. E. Marsden and A. Tromba. Vector Calculus. W. H. Freeman, New York, April I996. ISBN 0716724324 .

D. McWhorter and D. Sunada. Exact integral solutions for two-phase flow. Water Resources Research, 26(3):399-4I3, 1990. 


\section{BIBLIOGRAPHY}

A. Michel. A Finite Volume Scheme for Two-Phase Immiscible Flow in Porous Media. SIAM Journal on Numerical Analysis, 4I(4):I3OI-I3I7, 2003. ISSN 0036-I429.

I. D. Michev. Finite volume and finite volume element methods for nonsymmetric problems. $\mathrm{PhD}$ thesis, Texas A\&M University, College Station, 1996.

J. Molenaar. Multigrid methods for fully implicit oil reservoir simulation. In Proceedings Copper Mountain Conference on Multigrid Methods, I995.

V. V. Mourzenko, F. Yousefian, B. Kolbah, J.-F. Thovert, and P. M. Adler. Solute transport at fracture intersections. Water Resources Research, 38(I):I-I4, 2002.

I. Neretnieks. Solute transport in factured rock-Applications to radionuclide waste repositories. In J. Bear, C.-F. Tsang, and G. de Marsily, editors, Flow and contaminant transport in fractured rock, pages I-37. Academic Press, Inc., San Diego, I993.

I. Neretnieks, T. Eriksen, and P. Tähtinen. Tracer movement in a single fissure in granitic rock: some experimental results and their interpretation. Water Resources Research, I8(4): $849-858$, I982.

L. Neunhäuserer. Diskretisierungsansätze zur Modellierung von Strömungs- und Transportprozessen in geklïftet-porösen Medien. $\mathrm{PhD}$ thesis, Institut für Wasserbau, Universität Stuttgart, 2002.

L. Neunhäuserer, S. Gebauer, S. Ochs, R. Hinkelmann, R. Kornhuber, and R. Helmig. Equidimensional Modelling of Flow and Transport Processes in Fractured Porous Systems II. In S. M. Hassanizadeh, R. J. Schotting, W. G. Gray, and G. Pinder, editors, Computational Methods in Water Resources. Elsevier Science Publishers B.V., Amsterdam, 2002.

N. Neuss and C. Wieners. Criteria for the approximation property for multigrid methods in nonnested spaces. Mathematics of Computation, 2003. accepted.

J. Nitsche. Über ein Variationsprinzip zur Lösung von Dirichlet-Problemen bei Verwendung von Teilräumen, die keinen Randbedingungen unterworfen sind. Abh. Math. Sem. Univ. Hamburg, 36:9-I5, I97I.

J. Oden, I. Babuška, and C. Baumann. A Discontinuous hp Finite Element Method for Diffusion Problems. Journal of Computational Physics, I46:49I-519, 1998.

J. T. Oden and C. E. Baumann. A conservative DGM for Convection-Diffusion and NavierStokes Problems. In B. Cockburn, G. E. Karniadakis, and C. Shu, editors, Discontinuous Galerkin Methods: Theory, Computation and Applications, volume II of Lecture Notes in Computational Science and Engineering, pages 179-196. Springer-Verlag, Berlin, Heidelberg, New York, 2000.

OpenDX. The Open Source Software based on IBM's Visualization Data Explorer. http: //www.opendx.org/. 


\section{BIBLIOGRAPHY}

Y.-J. Park, K.-K. Lee, and B. Berkowitz. Effects of junction transfer characteristics on transport in fracture networks. Water Resources Research, 37(4):909-923, $200 \mathrm{I}$.

D. W. Peaceman. Interpretation of Well-Block Pressures in Numerical Reservoir SImulation. Transactions of the Societey of Petroleum Engineers of AIME, 1978.

P. Percell and M. Wheeler. A local residual finite element procedure for elliptic equations. SIAM J. Numer. Anal., I5(4):705-7I4, August 1978.

N. J. Price and J. Cosgrave. Analysis of Geological Structures. Cambridge University Press, I990.

R. Rannacher. Finite Element Methods for the Incompressible Navier-Stokes Equations. Preprint 99-37, IWR, Universität Heidelberg, SFB 359. Published in Special Issue of J. Math. Fluid Mech., September 1999.

M. Raw. Robustness of coupled algebraic multigrid for the Navier-Stokes equations. Technical Report 96-0297, AIAA, 1996.

W. Reed and T. Hill. Triangular mesh methods for the neutron transport equation. Technical report, Los Alamos Scientific Laboratory, 1973.

M. Renardy and R. C. Rogers. An Introduction to Partial Differentail Equations, volume I3 of Texts in Applied Mathematics. Springer-Verlag, Berlin Heidelberg New York, 1993.

H. Rentz-Reichert. Robuste Mehrgitterverfahren zur Lösung der inkompressiblen Navier-Stokes Gleichung: Ein Vergleich. PhD thesis, Universität Stuttgart, 1996.

A. Reusken. Convergence analysis of a multigird method for convection diffusion equations. Numerische Mathematik, 91:323-349, 2002.

B. Rivière. Discontinuous Galerkin methods for solving the miscible displacement problem in porous media. PhD thesis, The University of Texas at Austin, 2000.

B. Rivière and M. Wheeler. A Discontinuous Galerkin Method Applied to Nonlinear Parabolic Equations. In B. Cockburn, G. E. Karniadakis, and C. Shu, editors, Discontimuous Galerkin Methods: Theory, Computation and Applications, volume II of Lecture Notes in Computational Science and Engineering, pages 23I-244. Springer-Verlag, Berlin, Heidelberg, New York, 2000.

B. Rivière, M. Wheeler, and K. Banaś. Part II. Discontinuous Galerkin method applied to a single phase flow in porous media. Comput. Geosci., 4:337-349, 2000.

B. Rivière, M. Wheeler, and V. Girault. Improved energy estimates for interior penalty, constrained and discontinuous Galerkin methods for elliptic problems I. Computational Geosciences, 3:337-360, 1999.

ROCKFLOW. Theorie und Benutzeranleitung zum Programmsystem ROCKFLOW. Institut für Strömungsmechanik und Elektronisches Rechnen im Bauwesen, Universität Hannover, $1986-2003$. 


\section{BIBLIOGRAPHY}

E. S. Romm. Flow Characteristics of Fractured Rocks (in Russian). Nedra, Moscow, 1966.

J. Ruge and K. Stüben. Algebraic multigrid. In S. F. McCormick, editor, Multigrid Methods. SIAM, I987.

M. Sahimi. Flow and Transport in Porous Media and Fractured Rock: From Classical Methods to Modern Approaches. VCH, Weinheim, I995. ISBN 3-527-29260-8.

H. Schroll and A. Tveito. Local existence and stability for a hyperbolic-elliptic system modeling two-phase reservoir flow. Technical Report 136, Institut für Geometrie und Praktische Mathematik, RWTH Aachen, 1997.

C. Schwab. hp-FEM for Fluid Flow Simulation. In T. J. Barth and H. Deconinck, editors, High-Order Methods for Computational Physics, volume 9 of Lecture Notes in Computational Engineering, pages 325-438. Springer-Verlag, Berlin, Heidelberg, New York, 1999.

F. W. Schwartz and H. Zhang. Fundamentals of Groundwater. John Wiley \& Sons, Inc., New York, 2003. ISBN 0-47I-I3785-5.

T. Scott. Multi-grid methods for oil reservoir simulation in two and three dimensions. J. Comput. Phys., 59:290-307, 1985.

C. Shu. Total-variation-diminishing time discretizations. SIAM J. Sci. Stat. Comput., 9(6): IO73-IO84, 1988 .

C.-W. Shu. Essentially Non-oscillatory and Weighted Essentially Non-oscillatory Schemes for Hyperbolic Conservation Laws. In B. Cockburn, C. Johnson, C.-W. Shu, and E. Tadmor, editors, Advanced Numerical Approximation of Nonlinear Hyperbolic Equations, volume I687 of Lecture Notes in Mathematics, pages 325-432. Springer-Verlag, Berlin, Heidelberg, New York, 1998.

C.-W. Shu. High Order ENO and WENO Schemes for Computational Fluid Dynamics. In T. J. Barth and H. Deconinck, editors, High-Order Methods for Computational Physics, volume 9 of Lecture Notes in Computational Engineering, pages 439-582. Springer-Verlag, Berlin, Heidelberg, New York, 1999.

A. Silberhorn-Hemminger. Modellierung von Kluftaquifersystemen: Geostatistische Analyse und deterministisch-stochastische Kluftgenerierung. PhD thesis, IWS, Universität Stuttgart, 2002.

B. B. S. Singhal and R. P. Gupta. Applied Hydrogeology of Fractured Rocks. Kluwer Academic Publishers, Dordrecht, Boston, London, 1999.

L. Smith and F. W. Schwartz. An analysis of the influence of fracture geometry on mass transport in fractured media. Water Resources Research, 20(9):I24I-I252, 1984.

E. A. Sudicky and R. G. McLaren. FRACTRAN user's guide - an efficient simulator for twodimensional, saturated groundwater flow and solute transport in Porous or discretely fractured porous formations. Waterloo Centre for Groundwater Research, University of Waterloo, 1998. 


\section{BIBLIOGRAPHY}

R. Temam. Navier-Stokes Equations. North Holland Elsevier Science Publishers, Amsterdam, New York, Oxford, 1979.

V. Thomée. Galerkin finite element methods for parabolic problems. Number 25 in Springer Series in Computational Mathematics. Springer-Verlag, Berlin, Heidelberg, New York, 1997. ISBN 3-540-63236-0.

C.-F. Tsang and I. Neretnieks. Flow channeling in heterogeneous fractured rocks. Reviews of Geophysics, 36(2):275-298, 1998.

Y. W. Tsang and C. F. Tsang. Channel model of flow through fractured media. Water Resources Research, 23(3):467-479, 1987.

H. van der Vorst. Bi-CGSTAB: A fast and smoothly converging variant of BiCG for the solution of nonsymmetric line ar systems. SIAM J. Sci. Stat. Comp., I3:63I - 644, 1992.

C. J. van Duijn, J. Molenaar, and M. J. de Neef. Effects of capillary forces on immiscible twophase flow in heterogeneous porous media. Transport in Porous Media, 21:71-93, 1995.

P. Vaněk, J. Mandel, and M. Brezina. Algebraic multi-grid by smoothed aggregation for second and forth order elliptic problems. Computing, 56:179-196, 1996.

R. Verfürth. Multi-level algorithms for mixed problems II. Treatment of the Mini-Element. SIAM J. Numer. Anal., 25:285-293, 1988.

R. Verfürth. A Review of A Posteriori Error Estimation and Adaptive Mesh-Refinement Techniques. Advances in Numerical Mathematics. John Wiley \& Sons and B. G. Teubner, Chichester, New York, Stuttgart, Leipzig, 1996.

C. Wagner, W. Kinzelbach, and G. Wittum. A robust multigrid method for groundwater flow. Numer. Math., 75:523-545, 1997.

T. C. Warburton. Application of the discontinuous Galerkin method to Maxwell's eauations using unstructured polymorphic hp-finite elements. In B. Cockburn, G. E. Karniadakis, and C. Shu, editors, Discontinuous Galerkin Methods: Theory, Computation and Applications, volume II of Lecture Notes in Computational Science and Engineering. Springer-Verlag, Berlin, Heidelberg, New York, 2000.

T. C. Warburton and G. E. Karniadakis. A discontinuous Galerkin merhod for the viscous MHD equations. J. Comput. Phys., I52:I-34, 1999.

J. E. Warren and P. J. Root. The behavior of naturally fractured reservoirs. Soc. Pet. Eng. J., $3: 245-255,1963$.

P. Wesseling. An Introduction to Multigrid Methods. John Wiley \& Sons, Chichester, 1992. Reprinted by www.mgnet.org.

P. Wesseling. Principles of Computational Fluid Dynamics. Springer Series in Computational Mathematics. Springer-Verlag, Berlin, Heidelberg, New York, 200I. ISBN 3-540-67853-O. 


\section{BIBLIOGRAPHY}

M. Wheeler. An elliptic collocation finite element method with interior penalties. SLAM J. Numer. Anal., I5(I):I52-I6I, February 1978.

C. R. Wilson and P. A. Witherspoon. Steady state flow in rigid networks of fracures. Water Resources Research, IO(2):328-335, 1974.

P. A. Witherspoon, J. S. Y. Wang, K. Iwai, and J. E. Gale. Validity of cubic law for fluid flow in deformable rock fractures. Water Resources Research, 6(I6):IOI6-IO24, I980.

G. Wittum. On the robustness of ILU smoothing. SIAM J. Sci. Stat. Comput., Io:699-717, I989.

G. Wittum. On the convergence of multigrid methods with transforming smoothers. $\mathrm{Nu}$ merische Mathematik, 57:15-38, 1990.

A. Woodbury and K. Zhang. Lanczos method for the solution of groundwater flow in discretely fractured porous media. Advances in Water Resources, 24:62I-630, $200 \mathrm{I}$.

Y.-S. Wu, W. Zhang, L. Pan, J. Hinds, and G. S. Bodvarsson. Modeling capillary barriers in unsaturated fractured rock. Water Resources Research, 38(II), 2002.

J. Xu. Iterative methods by space decomposition and subspace correction: A unifying approach. SIAM Review, 34:58I-6I3, I992.

I. Yotov. A mixed finite element discretization on non-matching multiblock grids for a degenerate parabolic equation arising in porous media flow. East-West J. Numer. Math., 5: 2II-230, I997.

E. Zeidler. Applied Functional Analysis: Main Principles and Their Applications. Number 109 in Applied Mathematical Sciences. Springer-Verlag, New York, Berlin, Heidelberg, 1995. 
BIBLIOGRAPHY 


\section{INDEX}

Abdassah and Ershaghi (1986), 39, 127

Adler and Thovert (1999), 26, I27

Aizinger et al. (200I), $8 \mathrm{I}$, I27

Alexander (I977), 61, I27

Allaire (I997), 40, I27

Arbogast et al. (I990), 40, I27

Arbogast et al. (I99I), 40, I27

Arbogast (1989), 40, 127

Arbogast (1997), 40, 127

Arnold et al. (2002), $8 \mathrm{r}$, I27

Arnold (1979), 81, I27

Arnold (1982), $8 \mathrm{I}$, I27

Baca et al. (I984), 40, I27

Bank and Rose (1987), 51, I28

Bank and Wagner (I999), 68, 127

Barenblatt et al. (I960), 38, 39, I28

Barrett et al. (I994), 65, I28

Barton et al. (I987), I8, I28

Bassi and Rebay (1997a), 80, I28

Bassi and Rebay (1997b), 79, I28

Bassi and Rebay (2000), 79, I28

Bastian and Helmig (1999), 66, 70, I28

Bastian and Lang (2002), 8I, I28

Bastian and Reichenberger (2000), 8I, 88 , I29

Bastian et al. (1997), 46, 72, I28

Bastian (1996), 66, 69, I28

Bastian (I999), I3, 43, 48, 50, 54, 66, 69. 71, 128

Bastian (2002), 81, I28

Baumann and Oden (1999), 80, 85, I29

Baumann (1997), 80, 81, 85, I29

Bear et al. (I993), I3, I29

Bear (1972), I3, 24, 25, 48, 49, I29

Bear (I993), II7, I29
Berkowitz et al. (1988), 40, I29

Berkowitz (2002), 26, 29, I29

Bertels et al. (200I), 33, I29

Bey and Wittum (1997), 68, I29

Bey (1998), 51, 53, 66, 68, I29

Binning and Celia (1994), 45, I29

Bourgeat (I997), 40, I29

Braess (1992), 62, 63, I29

Braess (1995), 68, I29

Brakhagen and Fogwell (1990), 65, I30

Bramble et al. (I99I), 67, I30

Bramble (1993), 65, I30

Brenner and Sung (1997), 68, 130

Briggs et al. (2000), 65, 130

Brinkman (1947), 25, I30

Brooks and Hughes (I982), 45, 130

COVISE (), 77, I32

Castillo (2002), $8 \mathrm{~B}$, I3O

Celia et al. (I990), 45 , I3O

Chavent and Jaffré (1978), 47, 130

Chavent (1976), 46, I30

Chavent (I98I), 46, I30

Chen et al. (1995a), 79 , I30

Chen et al. (1995b), 79 , I30

Chen (1989), 39, I30

Ciarlet (1978), 40, I3I

Class (200I), 30, I3I

Clemo and Smith (1989), 39, I3I

Cockburn and Shu (1989), 80, I3I

Cockburn and Shu (I99I), 80, I32

Cockburn and Shu (1998a), 80, I32

Cockburn and Shu (I998b), 80, I32

Cockburn et al. (I989), 80, I3I

Cockburn et al. (I990), 80, I3I

Cockburn et al. (2000a), 79, I3I 
Cockburn et al. (2000b), 45, I3I

Cockburn et al. (2000c), 79, I3I

Cockburn (I998), 45, I3I

Cockburn (I999), 45, I3I

Courant et al. (I928), 48 , I32

Curtiss and Hirschfelder (1952), 58, I32

Darcy (I856), 23, I32

Dawe et al. (I992), 36, I32

Delfour et al. (198I), 80, I32

Dendy Jr. (1987), 65, I32

Doering and Gibbon (I995), I5, I32

Douglas jr. (1983), 46, I32

Dryja et al. (1996), 67, I32

Durlofsky (I993), 44, I32

Durlofsky (1994), 89, 9I, I33

Eaton and Bixler (1987), 40, I33

Eriksson et al. (1985), 80, I33

Eriksson et al. (I996), 61, 80, I33

Evans (1998), 49, I33

Ewing (I983), II, 43, I33

Ewing (1991), 45, I33

Forchheimer (I9OI), 25, I33

Forsyth (I99I), 45, I33

Fuchs (1999), 75, I33

Glimm et al. (I98I), 49, I33

Glimm et al. (I983), 49, I33

Golub and van Loan (I996), 63, 65, I33

Gopalakrishnan and Kanschat (2003), 81, I33

Gray and Lee (1977), 31, I33

Gureghian (1975), 40, I34

Hackbusch (1985), 65, 67, I34

Hackbusch (I989), 5I, I34

Hackbusch (I994), 64, 65, 70, I34

Hackbusch (1997), 68, I34

Hairer and Wanner (1996), 58,53

Helmig and Huber (1996), 44, I34

Helmig (1993), 40, I34

Helmig (I997), I3, 30, 31, 33, 44, 45, 50, I34

Hemker and van Raalte (2002), 81, 88, I34

Hemker et al. (2002a), 88, I34

Hemker et al. (2002b), 88 , I34

Hill and Thomas (1985), 39, I34
Horn and Johnson (1985), I24, I35

Horn and Johnson (1991), I24, I35

Hornung (1997), 24, 32, 38, I35

Hou and LeFloch (1994), 44, 45, I35

$\mathrm{Hu}$ and Shu (2000), 79, $\mathrm{I35}$

Ippisch (200I), 24, 30, I35

J. S. Y. Wang and T. N. Narasimhan (1985), 40,135

Jänich (2003), I23, 135

Jakobs et al. (2003), 63, I35

Jakobs (2003), 29, I35

Jamet (I978), 80, I35

Jarsjö and Geller (I996), I8, I35

Johnson et al. (I990), 80, I35

Kazemi (1969), 39, I35

Kroener and Luckhaus (1984), 47, I35

Kueper and Frind (1988), 49, I35

Kueper et al. (1989), 36, I36

Lang (2000), 66, 69, 70,136

Laubach (1992), I8, I36

Le Veque (I992), 48, I36

Le Veque (2002), 44, 45, 48, I36

Lenhard et al. (1989), 33, I36

Lesaint and Raviart (1974), 80,136

Lions (I968), 80, 136

Lomize (I95I), 25, I36

Louis (1969), 25, I36

Lucia (1999), I36

Ma et al. (1999), 33, I36

Marsden and Tromba (1996), I23, I36

McWhorter and Sunada (I990), 50, I36

Michel (2003), 51, I36

Michev (1996), 45, 51, 53, 57, I37

Molenaar (I995), 65, I37

Mourzenko et al. (2002), 26, I37

Neretnieks et al. (1982), 26, I37

Neretnieks (1993), 26, I37

Neunhäuserer et al. (2002), 75, I37

Neunhäuserer (2002), 92, I37

Neuss and Wieners (2003), 69, I37

Nitsche (197I), 80, 137

Oden and Baumann (2000), 79, 137

Oden et al. (I998), 80, 81, 85, I37

OpenDX (), 77, I37 
Park et al. (200I), 26, I37

Peaceman (1978), IO6, I38

Percell and Wheeler (1978), $8 \mathrm{I}, \mathrm{I38}$

Price and Cosgrave (1990), I7, I38

ROCKFLOW (1986-2003), 40, I38

Rannacher (I999), 60, I38

Raw (1996), 68, 138

Reed and Hill (I973), 79, I38

Renardy and Rogers (1993), 48, I38

Rentz-Reichert (I996), 68, I38

Reusken (2002), 68, 138

Rivière and Wheeler (2000), $8 \mathrm{r}, \mathrm{I3} 8$

Rivière et al. (I999), $8 \mathrm{I}, \mathrm{I3} 8$

Rivière et al. (2000), $8 \mathrm{I}, \mathrm{I} 38$

Rivière (2000), $8 \mathrm{I}, \mathrm{I} 38$

Romm (I966), 25, I38

Ruge and Stüben (I987), 68, I39

Sahimi (I995), I3, 25, 38, I39

Schroll and Tveito (I997), 47, I39

Schwab (1999), 45, I39

Schwartz and Zhang (2003), I39

Scott (I985), 65, I39

Shu (1988), 80, I39

Shu (1998), 44, I39

Shu (1999), 44, I39

Silberhorn-Hemminger (2002), 74, I39

Singhal and Gupta (I999), I3, I5, I7, I8, 20, 22, 25, 39, 139

Smith and Schwartz (1984), 26, I39

Sudicky and McLaren (1998), 40, I39

Temam (I979), I5, I39

Thomée (1997), 50, 58, 6r, 80, I40

Tsang and Neretnieks (I998), 26, I4O

Tsang and Tsang (1987), 26, I40

Vaněk et al. (I996), 68, I40

Verfürth (1996), 69, I4O

Verfürth (1988), 65, I40

Wagner et al. (I997), 67, I40

Warburton and Karniadakis (1999), 79, I40

Warburton (2000), 79, I40

Warren and Root (I963), 39, I4O

Wesseling (1992), 65, I40

Wesseling (200I), 65, I40

Wheeler (1978), 8I, I4O
Wilson and Witherspoon (1974), 40, I2I I4I

Witherspoon et al. (I980), 25, I20, I4I

Wittum (I989), 68, I4I

Wittum (I990), 65, I4I

Woodbury and Zhang (200I), 40, I4I

Wu et al. (2002), 36, I4I

$\mathrm{Xu}$ (1992), 65, I4I

Yotov (I997), 47, I4I

Zeidler (1995), 26, I4I

van Duijn et al. (I995), 36, 37, 45, II5, I4O

van der Vorst (1992), 92 , I4O

Douglas Jr. and Russel (I982), 44, I32

Douglas Jr. et al. (I959), 44, I32

A-stable, 60

strongly, 60

absolute permeability, 24

adhesive forces, 26

anisotropic, 22, 67

anisotropic problems, 67

anisotropy, 24

asperity, I8

average, 84

average pressure, 46

backward Euler, 59

backward Euler scheme, 60

Barenblatt, 49

basis function, 82

Baumann-Oden method, 85

bedding plane, $I 5$

Brooks-Corey, 33, 35, 37

Brooks-Corey capillary pressure function, 33

Brooks-Corey relative permeability functions, 34

bubbling pressure, 30

Buckley-Leverett equation, 47

capillary force, 26

capillary pressure, 28, 32, 36, 37, 4I

capillary pressure function, $5 \mathrm{II}$

Brooks-Corey, 33

Van Genuchten, 33 
causes of fracturing, 17

CFL condition, 48

channeling, 18,26

characteristic methods, 44

cohesive forces, 26

computer program, II

contact angle hysteresis, 30

continuum model, 37

convection dominated, 67

convection-diffusion problems, 67

Courant-Friedrichs-Lewy condition, 48

Crank-Nicholson scheme, 60

crossing fractures, I6

cut-off fracture aperture, 29

DAE, 59

Darcy velocity, 23

Darcy's law, 23, 32

defect, 64

degenerate parabolic, 47

density, 24

differential algebraic equations, 59

discontinuities, 36

discontinuous Galerkin method, 61, 79, 80

for fractures, 86

discrete fracture network model, 40

discrete model, 37, 40

double-porosity model, 38

drainage, 29

dual-permeability model, 39

dynamic viscosity, 24

effective aperture, I2I

effective pore space, I4

effective porosity, 20,24

effective saturation, 33

elements

$$
\text { shell, } 40
$$

elevation head, 23

ELLAM, 45

ENO, 44

entry pressure, 30,37

equation of state, 24,38

equivalence model, 38 equivalent model, 37

Euler scheme

backward, 60

explicit, 60

forward, 60

implicit, 60

Eulerian-Lagrangian localized adjoint method,

45

exchange term, 39

exchange terms, 38

explicit Euler scheme, 60

extended capillary pressure condition, 37 , 45

fault, I6

fingering, 49

finite volume method, $5 \mathrm{~s}, 88$

first-order fractures, $I 7$

fissured media, 39

foliation, I6

formulation

$$
\begin{aligned}
& \left(p_{g}, S_{w}\right), 5 \mathrm{I} \\
& \left(p_{w}, S_{g}\right), 50
\end{aligned}
$$

$\left(\mathrm{p}_{w}, \mathrm{p}_{\mathrm{g}}\right)$, sI

global pressure, 47

Phase pressure-Saturation, 50

pressure-pressure, 5I

forward Euler scheme, 60

fractional flow function, 48

fractional-step- $\theta$ scheme, $6 \mathrm{I}$

fracture, 16

open, 25

types of, 17

fracture characterization, I7

fracture intersections, 26

fracture network, 38

fractures

first-order, $I 7$

non-systematic, 17

open, I6

primary, 40

second-order, I7

systematic, I7

fracturing 
causes of, I7

frontal mobility ratio, 48

geometric multigrid, 68

global pressure formulation, 47

gravity, 24

hanging nodes, 82

Hele-Shaw analog, 25, II

heterogeneous, 22

homogeneous, 22

hybrid model, 40

hydraulic conductivity, 23

hysteresis, 29

ideal gas law, 24

imbibition, 29

IMPES, 44, 65

implicit constraint, 59

implicit Euler scheme, 60

incompressible, 24

interface problems, 67

interior penalty, $8 \mathrm{I}$

intermediate wetting phase, 28

intrinsic permeability, 24

IP, $8 \mathrm{I}$

isotropic, 22

joint, I6

jump, 84

Krylov subspace methods, 65

load balancing problem, 70

local cubic law, 25

local discontinuous Galerkin method, 80

macroscopic fluid mass conservation, 24

macroscopic scale, IS

mathematical formulation, II

McWhorter problem, 50

method of lines, 58

microfractures, 22

microscale, 26

microscopic scale, Is

MMOC, 44 mobility

$$
\begin{aligned}
& \text { phase, } 46 \\
& \text { total, } 46
\end{aligned}
$$

model

continuum, 37

discrete, 37, 40

double-porosity, 38

dual-permeability, 39

equivalent, 37

hybrid, 40

multi-continuum, 38

modified method of characteristics, 44

molecular scale, I4

Moore-Penrose pseudo inverse, I24

multi-continuum model, 38

multi-porosity models, 39

multigrid

geometric, 68

multigrid method, 65

Navier-Stokes equations, I5, 24, 6I, II7

NIPG, $8 \mathrm{I}, 85$

non-symmetric interior penalty method, $8 \mathrm{I}$

non-systematic fractures, 17

nonlinear multigrid method, 63

nonwetting fluid, 27

numerical flux, 80

numerical model, II

one-step- $\theta, 59$

open fracture, 25

open fractures, I6

parallel plate model, 25, 29, II7

parallelization, 69

penalty terms, 80

permeability

absolute, 24

intrinsic, 24

relative, 32

phase mobility, 46

phase permeability, 32

Phase pressure-Saturation formulation, 50

physical model, II

point-block ordering, 70 
Poisson equation, 88

pore space, $I 4$

Porosity, I9

porosity, 2I, 24

effective, 20,24

primary, 20

secondary, 20

total, 20

porous medium equation, 49

PowerBook, IO8

PowerMac, IO9

preconditioner, 65

pressure, 24

average, 46

bubbling, 30

capillary, 28, 36,37

entry, 30,37

threshold, 30

pressure head, 23

pressure-pressure formulation, $5 \mathrm{I}$

primary fractures, 40

primary porosity, 20

prolongation, 66

relative permeability, 32, 34, 4I

relative permeability functions, 34

Brooks-Corey, 34

Van Genuchten, 34

relaxation methods, 64

representative elementary volume, 20, $2 \mathrm{I}$

residual saturation, 30, 32

restriction, 66

truncated, $7 \mathrm{I}$

REV, 20

robust, 67, 68

roughness, I20

Runge-Kutta scheme

singly diagonally implicit, $6 \mathrm{I}$

saturation, $3 \mathrm{I}$

effective, 33

scheme

fractional-step- $\theta, 6 \mathrm{I}$

SDIRK, 6I second-order fractures, 17

secondary porosity, 20

semi-coarsening, 68

shear, I6

shell elements, 40

singly diagonally implicit Runge-Kutta scheme, 6 I

smoother, 66,87

solid matrix, I4

stability domain, 60

strongly A-stable, 60

subdivision, 52

surface tension, 27

systematic fractures, $I 7$

test function, 82

threshold pressure, 30

time step size, IIO, III

total fluid velocity, 46

total mobility, 46

total porosity, 20

total velocity, 48

totally fissured media, 39

triangulation, 52

triple-porosity model, 39

truncated restriction, $7 \mathrm{I}$

types of fractures, 17

unconnected pores, I4

unit square, 88

upwind, 80

Van Genuchten, 33, 35, 37

Van Genuchten capillary pressure function, 33

Van Genuchten relative permeability func-

velocity tions, 34

total fluid, 46

viscosity, 24

dynamic, 24

void space, I4

WENO, 44

wettability, 27 
INDEX

wetting fluid, 27 